\title{
TOWARD MARKETING AS A DISCURSIVE PRACTICE: RETHINKING THE DISCIPLINE'S PHILOSOPHICAL FOUNDATIONS
}

\section{By}

\section{David Murray Stewart}

\author{
A thesis \\ submitted to Victoria University of Wellington \\ in fulfilment of the requirements for the degree of \\ Doctor of Philosophy \\ in Marketing
}

Victoria University of Wellington 
Declaration

Except for commonly understood and accepted ideas, or where specific reference is made, the work in this dissertation is my own and includes nothing which is the outcome of work done in collaboration. The work has not previously been submitted in part or in whole to any university for any degree or other qualification. In accordance with the regulations of Victoria University the dissertation contains no more than 100,000 words of text. 


\section{Abstract}

It has been claimed that marketing needs to create a new 'paradigm'. Following a critical analysis of marketing's philosophical foundations the thesis argues that the discipline should instead seek to build upon the 'linguistic' or 'postmodern' turns in the philosophy of science: a turn that has already been embraced by seemingly related disciplines like sociology, history and anthropology, for example. By connecting to this development, and the approaches that it has engendered such as narrative theory, neo-pragmatism and the notion of a discursive practice, marketing will be better able to develop more useful knowledge or practical wisdom

('phronesis'). A new approach to the generation of marketing knowledge based on praxis will enable three problems that have become apparent in the field over the past decade to be overcome. First, the debate whether marketing is an art or a science can be resolved. Second, the gap between the marketing discipline and practitioners can be closed. Third, the stagnant state of marketing theory can be invigorated. An exemplary case study, which explores the launch of Air New Zealand's 'Domestic Express' service, demonstrates how such an approach to marketing might be operationalised and the added value that it could provide, both to marketing theorists and marketing practitioners. 


\section{Acknowledgements}

I am grateful to many people for their help, both direct and indirect, in writing this dissertation. It has been a long, but rewarding journey and would not have been possible without their ongoing encouragement and support.

I would like to acknowledge the debt I owe to faculty at Victoria University of Wellington, especially my supervisor, Professor Bob Gregory. Bob has been the source of helpful advice, support and seemingly limitless patience. I am grateful for his guidance and friendship. I would also like to thank my second supervisor, Professor Stephen Cummings, for his valuable input into the research.

The on-going encouragement and interest shown by members of the wider University community has been invaluable. In particular I would like to thank Professor John Brocklesby, Adam Weaver, Jane Bryson, Noelle Donnelly and Professor Gordon Anderson.

Many thanks go to my children, Georgina and James, for understanding the sacrifices required whilst undertaking the $\mathrm{PhD}$. Thanks also to my partner Splodge for believing in me and for her support.

Finally, I would like to thank Air New Zealand for providing material for the case and in particular the staff members who gave their time and effort to participate in the study. 


\section{Contents}

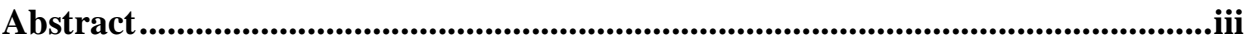

Acknowledgements .............................................................................................................iv

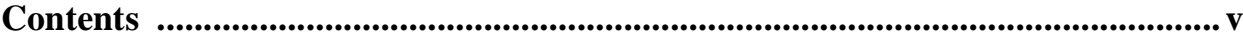

List of Tables....................................................................................................................viii

List of Figures ...........................................................................................................viii

Chapter 1: Introduction: Dilemmas in Marketing Management............................1

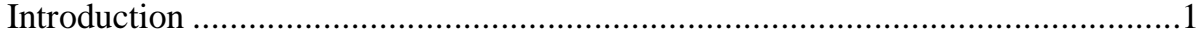

Schools of Thought ............................................................................. 3

The Dominant School: Marketing Management .............................................6

Art or Science ................................................................................. 10

Academic Research and Practitioner Relevance ....................................... 13

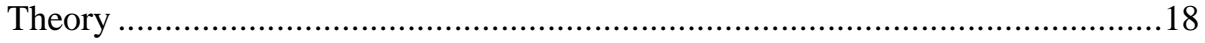

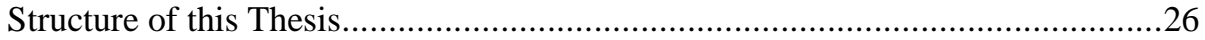

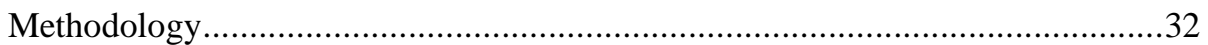

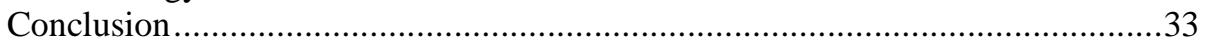

Chapter 2: Beyond Paradigm Wars ....................................................................... 34

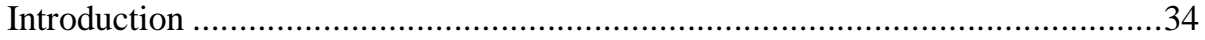

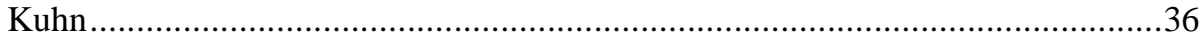

Burrell and Morgan ............................................................................... 42

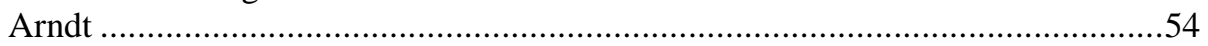

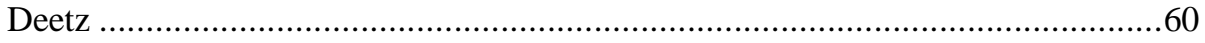

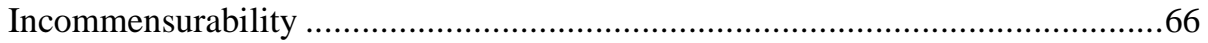

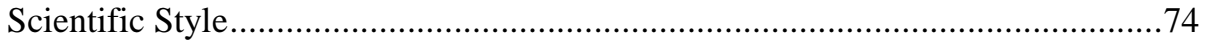

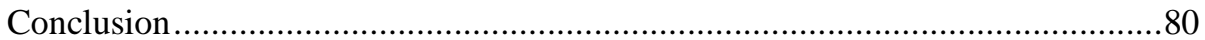

Chapter 3: The Dominant Paradigm ....................................................................... 82

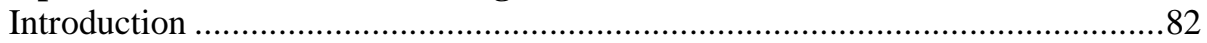

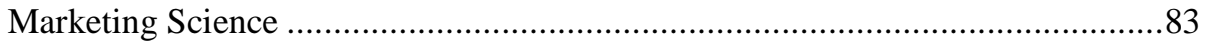

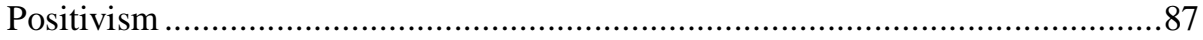

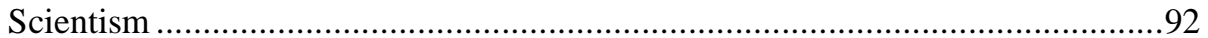

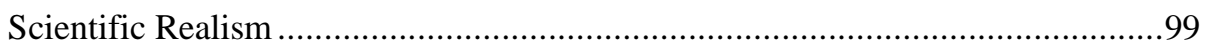

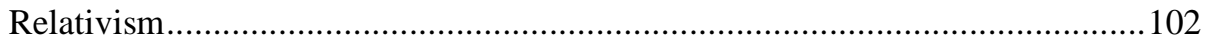

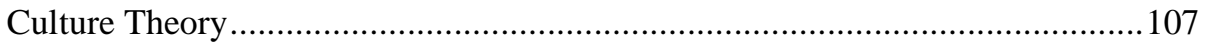

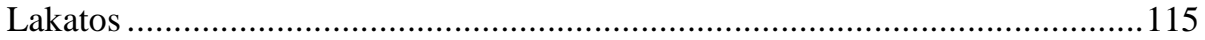

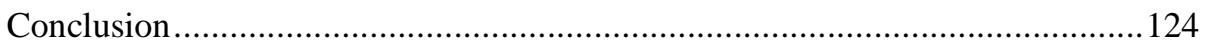

Chapter 4: Understanding Postmodernism ............................................................ 126

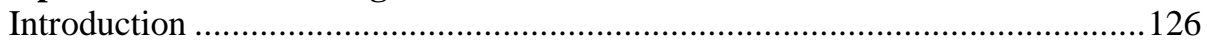

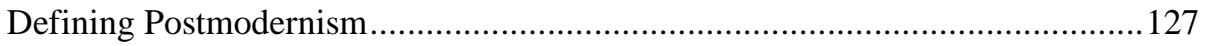

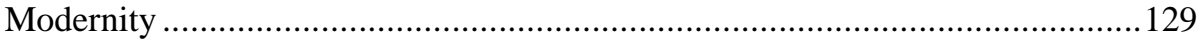

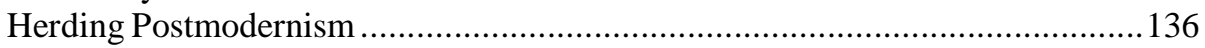

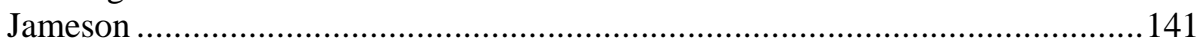

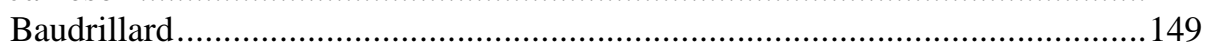

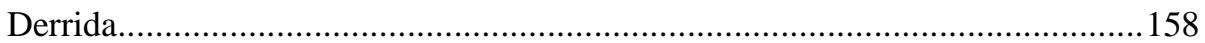




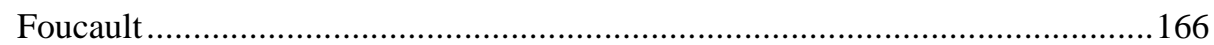

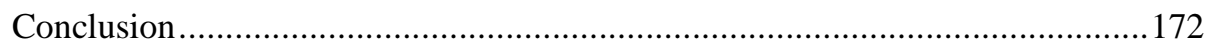

Chapter 5: Neo-Pragmatism............................................................................ 175

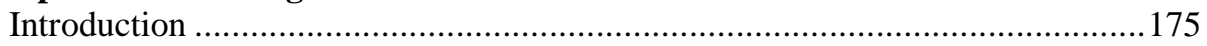

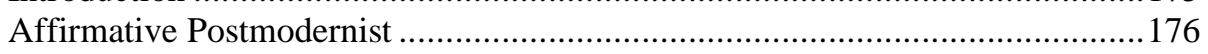

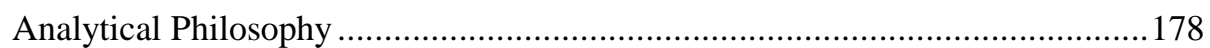

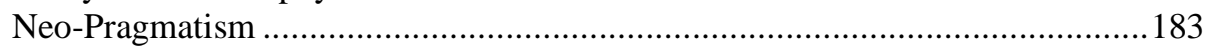

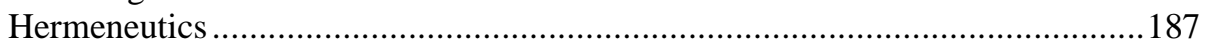

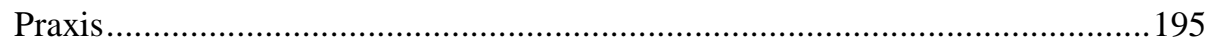

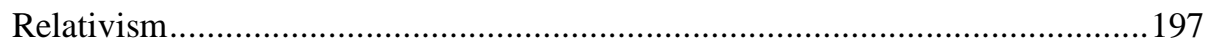

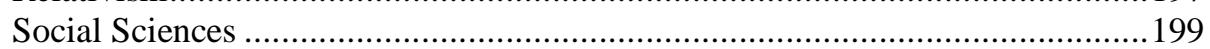

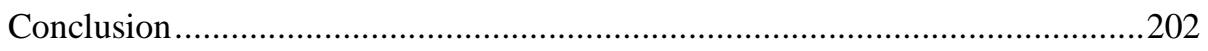

Chapter 6: Narrative Theory ............................................................................205

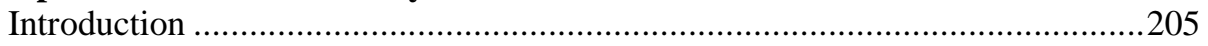

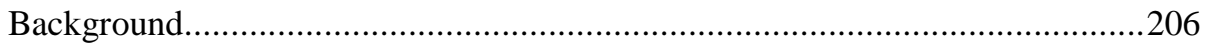

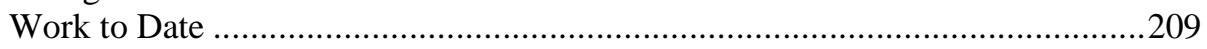

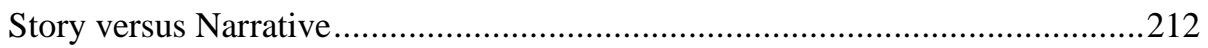

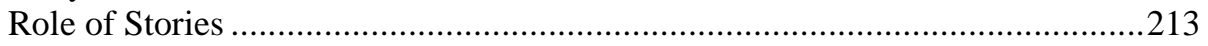

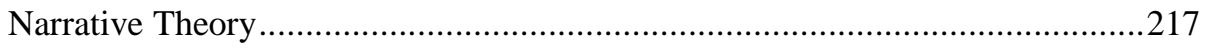

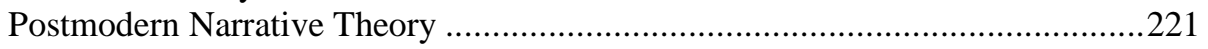

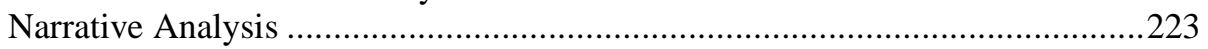

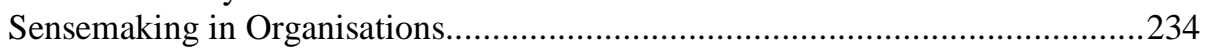

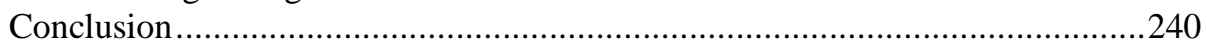

Chapter 7: Exemplary Case Study - Air New Zealand Domestic Express....... 241

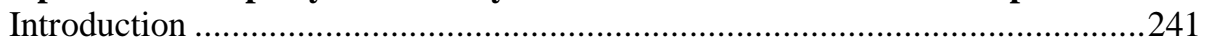

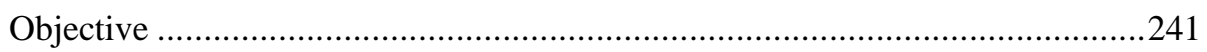

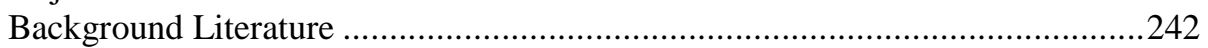

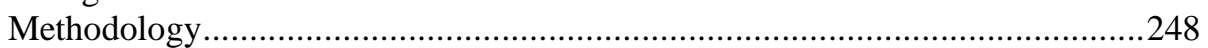

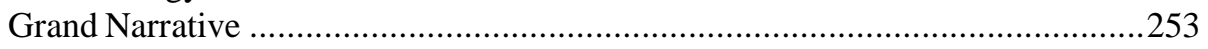

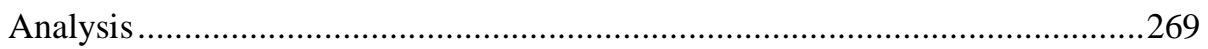

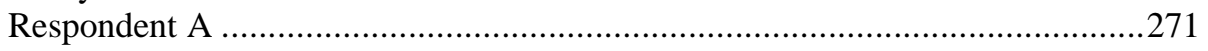

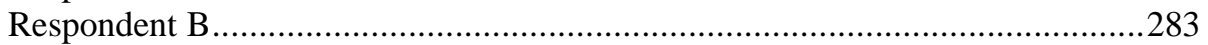

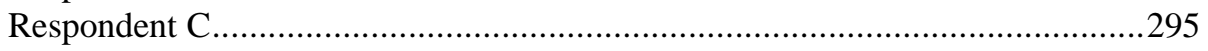

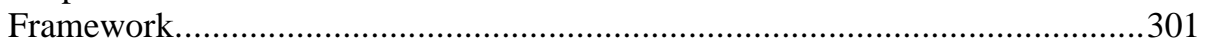

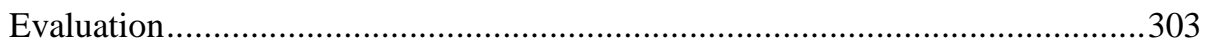

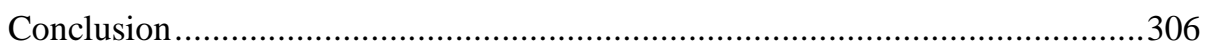

Chapter 8: Conclusion - Contribution of a New Discourse ....................................307 307

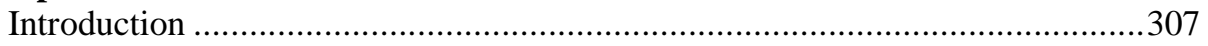

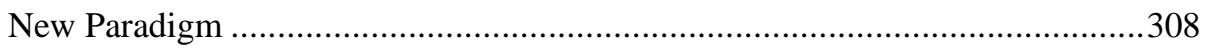

Implications for Research.........................................................................

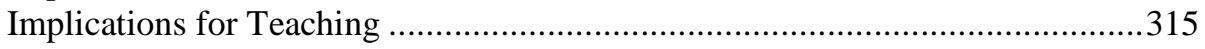

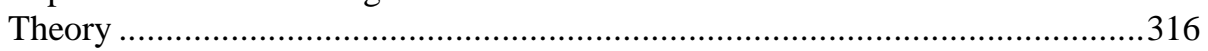

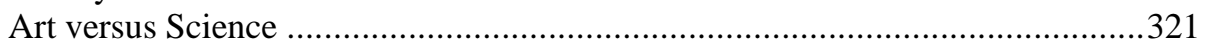

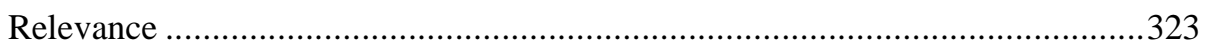

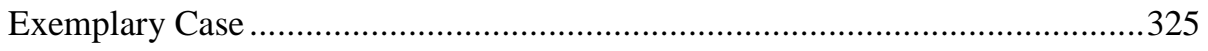

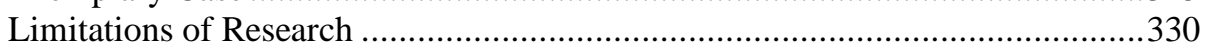

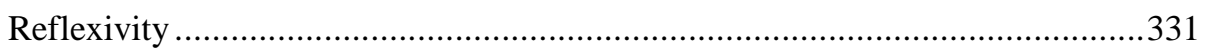

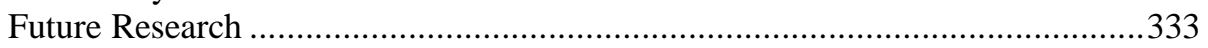

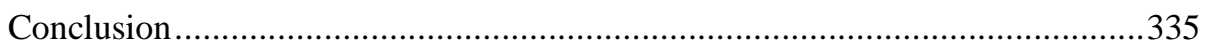

References ..................................................................................................................... 337 
Appendices

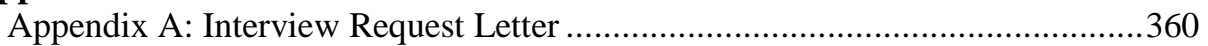

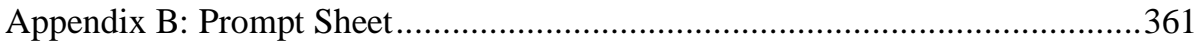

Appendix C: Airline Best Practice ....................................................................363

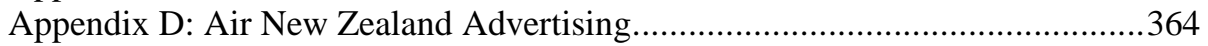

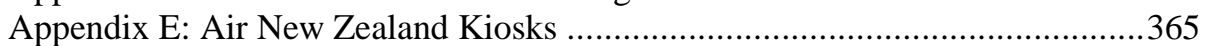




\section{List of Tables}

Table 1: Key Differences between Scientific and Artistic Research ................. 12

Table 2: Common Divisions Affecting Organisational Inquiry.......................... 51

Table 3: Ten Grand Narratives of Enlightenment Knowledge …...................... 225

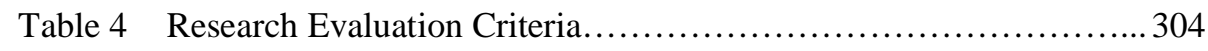

Table 5 Comparison of Research Approaches................................ 312

\section{List of Figures}

Figure 1: Sociological Paradigms and Organisational Analysis ......................... 46

Figure 2: Paradigms and Metaphors in Marketing ...........................................56

Figure 3: Characteristics of the Local/Emergent-Elite/A Priori Dimension....... 62

Figure 4: Characteristics of the Consensus-Dissensus Dimension .................... 64

Figure 5: Contrasting Dimensions from the Metatheory of Representational

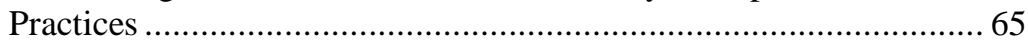

Figure 6: Four Scientific Styles................................................................. 76

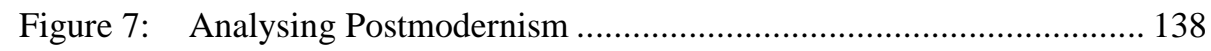

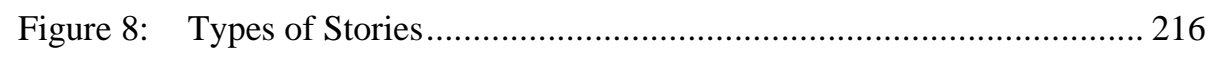

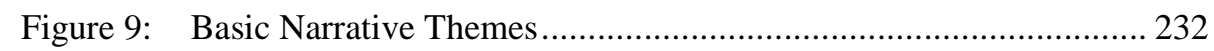

Figure 10: Strategic Balance Matrix............................................................ 246

Figure 11 Narrative Themes applied to Air New Zealand .............................. 302

Figure 12: Marketing Theories.................................................................... 319 


\section{Chapter 1: Introduction: Dilemmas in Marketing Management}

\section{Introduction}

Marketing as an academic discipline is facing a crisis, and at the centre of this crisis is the doubt concerning the necessary constructs for the generation of knowledge. There are a number of issues that have brought about this crisis, for instance, the disillusionment with the 'marketing concept'; the proliferation of different marketing schools of thought; the globalisation of markets; rapid development of technology; and the increased dominance of service economies. Also, from some quarters there has been disquiet about the dominant philosophy of science approach adopted by mainstream marketing academic endeavour. To overcome these problems it has been suggested that marketing needs to adopt a new paradigm. Gronroos (1994) states that a paradigm shift in marketing is needed if marketing is going to survive as a discipline. Other authors, such as Buttle (1994), Achrol (1997) and Brodie, Coviello, Brookes \& Little (1997) also reiterate the claim that a new paradigm is required to take into account relationship marketing. Day \& Montgomery (1999) calls for the marketing discipline to adopt a new paradigm, such as interactive or relationship marketing. They acknowledges that the marketing mix (product, place, price and promotion) is a useful framework for analysing some marketing situations, but it is too narrow and restrictive to cover all marketing dimensions. They note that pressures on marketing to adopt a new paradigm come from the growth of the knowledge economy, with the consolidation of industries contrasted with fragmenting markets due to media and channel proliferation, and also the growth of mass customisation. 
Despite the above problems faced by the marketing discipline, the thesis will argue that whast is required is for marketing to adopt the linguistic turn in modern philosophy. The thesis will first explore the philosophical underpinnings of marketing management, and then examine the linguistic turn in modern philosophy, represented by postmodernism. This turn has increased the focus on language (Alvesson and Karreman). Represented by postmodernism the complexities of language have been highlighted. According to Alvesson and Karreman (2000) the linguistic turn has been prevalent for some time in sociology, social psychology, communication theory, and cultural anthropology. Within the general marketing literature the topic of postmodernism has been examined from a cultural perspective by a number of authors (O’Shaughnessy \& Holbrook, 1988; Brown, 1993; 1994;1995a;1999; Firat,Dholakia \& Venkatesh, 1995; Firat and Venkatesh, 1995; Hackley, 2001; O'Shaughnessy \& O'Shaughnessy, 2002), especially in the field of consumer behaviour. However, to date there has not been the development of a research programme based on the linguistic turn, showing the application of a postmodern research protocol on a marketing management problem.

The argument of this theoretical thesis is that marketing management needs to adopt the linguistic turn in modern philosophy. The thesis will explore the philosophical underpinnings of marketing management and explain why the linguistic turn should be adopted, followed by an exemplary case study to indicate how this research programme could be operationalised.

The thesis will also examine the effect of the new research programme on three dominant issues within the marketing management school, namely the concern 
surrounding marketing's status as an art or a science; second, the widening gap between academic research and practitioner relevance; and third, the lack of theory development in the marketing domain.

This chapter will begin by outlining the different schools of thought in the marketing discipline and explain why the marketing management should has been chosen to frame the thesis. This will then be followed by an exploration of the three issues outlined above, whilst the second part of the chapter will provide a synopsis of the remaining chapters of the thesis.

\section{Schools of Thought}

A problem faced by marketing is the proliferation of different marketing schools of thought, challenging the idea of an inclusive sphere of marketing knowledge. Sheth and Gardner (1982) reviewed the development of marketing over the last 50 years and found that, whilst marketing was an offshoot of economics, there had been a change from an emphasis on the economic exchange to the value exchange. They also noted that psychological understanding of the buyer was used in place of the economic perspective to build a more realistic theory of marketing.

Sheth and Gardner also identified six marketing schools that have developed over the same period. It would be useful at this stage to briefly outline the six schools. First is the macro marketing school, which analyses marketing activity from a societal perspective as a whole, rather than marketing activity being analysed at the firm level. This school of thought basically sees an interaction between society and marketing, rather than one more dominant than the other. 
The second school is that of consumerism, which arose from the need for there to be an advocacy role to protect the rights of the consumer. This movement grew when surpluses were generated after the second world war, and has grown since. The arch advocate in the United States was Ralph Nader who campaigned vigorously for consumer rights. It could be argued that the book by Naomi Klein (2000), 'No Logo', is an extension of this school.

Another school is the systems approach, which emphasises both the supply and demand side of marketing. This school grew in the 1960s and 1970s due mostly with the increase in computer capability. The use of simulation models is used to predict options available to consumers. This school is likely to grow due to the increased availability of computer capability.

The buyer behaviour school is perhaps one of the most dominant schools in the academic marketing arena, and came to the fore in the early 1950s, with the emphases on the packaged goods market. This school has applied psychological principles to consumer behaviour. By transferring social science methodology to the area of marketing they have brought the status of scientific enquiry to the discipline. Moving away from the merely descriptive approach based on demographics and market size statistics, this school has been able to develop specific constructs such as brand loyalty, attitude, intentions, and information processing. The school has also borrowed theory from social psychology and sociology. However, it will be argued in Chapter Three that buyer behaviour should not be considered to be part of marketing, but a separate academic discipline. 
Behavioural organisation is a school that has focused on business to business marketing. In particular there has been an emphasis on the channels of distribution from perspectives of power, conflict, and interdependence. One of the problems faced by this school is the difficulty of obtaining data regarding the interactions between businesses, compared with the relative ease in gaining data from an individual organisation. Another problem is that observed relationships are dynamic in nature, thereby making it hard to create generalisations.

Finally, strategic planning is the last of the six schools described by Sheth and Gardner (1982). This school attempts to balance external constraints with internal factors based on resource allocation. The external environmental constraints include such factors as competition, technology, political, and economic issues. The interesting point that can be noted about this school is that the majority of contributions have come from consulting groups, for example, the Boston Consulting Group, and not from marketing academics. Such 'practical' contributions are based on either an implicit cash flow maximisation or a form of the capital asset pricing model. However, because of the type of contribution, there is little conceptual development that can be used as a guide for future research.

The existence of the above 'Schools of Marketing' indicates that the discussion is primarily about how organisations relate to the market by way of the exchange process. However, Baker (2000) has noted, the main perspective dominating the marketing discipline is that of marketing management. 


\section{The Dominant School: Marketing Management}

Marketing management was developed in the 1960's, and has been dominant within marketing for over 30 years. The main proponents were Kotler and McCarthy who based their approach on a micro-economic framework (Dixon \& Wilkinson, 1989), with profit maximization as the central principle. The major emphasis is on problem-solving and decision-making around the central management areas of analysis, planning and control. Frameworks have been borrowed from other disciplines, such as psychology, economics, and sociology, and the research agenda has been based on a positivist paradigm (Baker, 2000), with measurement and analysis being the primary focus, which has meant an over reliance on statistics (Brown, 1999; Hackley, 2001).

The underlying assumptions of microeconomics has demand and cost curves as independent variables, so opportunities for marketing management are based on manipulating different demand conditions. The overall thrust of this approach is the control of demand management for the product or service being offered. This can be achieved by altering the different aspects of the marketing mix, namely price, product, place and promotion.

To achieve demand management the marketing management school divides an organisation into three levels of business. The first level of business is centred on the corporation and one central concern is about the culture of the organisation. It is at this level that the marketing concept is positioned. The next level is the strategic business unit (SBU), where the strategy of the business focuses on the product mix and how the SBU is positioned in the marketplace. The third level is that of tactics 
where the goal is demand stimulation by the manipulation of the components of the marketing mix.

Central to the marketing management school is the exchange process which is based on the joint action of the buyer and seller. Such an action relies on three types of outputs, namely contact, material transformation, and contract (Dixon \& Wilkinson 1989). The first output means that contact must be made between the buyer and seller involving two way communications. The second output involves the transfer of a good or service. Finally, a contract is established whereby the ownership and use of the goods and services being exchanged takes place. This may involve the transfer of money, but not in all cases, for example, the marketing of a sports event where participants enter at no cost.

The problem with the marketing management school is that it is production oriented thereby being more about what marketers can 'do' to consumers than with any type of reciprocal arrangement. Following from this, the focus is short-term and transactional based rather than being concerned with any maintenance of a long-term relationship with the customer. ${ }^{1}$ Also the main area of analysis and focus is that of the fast moving consumer goods (fmcg) market. Another concern is that whilst the marketing mix is utilised, other aspects affecting consumer demand are ignored, such as environmental analysis and competitive forces.

It would seem that over the last two decades marketing management has expanded into different areas. For instance, services marketing has adopted an additional 3 P's

\footnotetext{
1 Refer to Gronroos re relationship marketing
} 
(Magrath, 1986), where physical evidence, process, and personnel are added to accommodate the different nature of service marketing. Vargo and Lusch (2004) argue that the new dominant logic for marketing is based on intangible resources, value creation and the management of relationships. They argue that service provision will replace the economic exchange model based on a goods centred approach.

Another diverse approach to marketing management is that of relationship marketing, championed by Christian Gronroos (1990). In his 1994 article 'From Marketing Mix to Relationship Marketing: Towards a Paradigm Shift in Marketing', he argues that there has been a move away from traditional marketing. He claims that the marketing mix, commonly known as the $4 \mathrm{P}$ 's, no longer applies to the modern world as it was developed in North America in the 1950's for the 'fast moving consumer goods' market where there was a large domestic market with a unique media structure and a competitive distribution system.

Also non-profit organisations have adopted the marketing concept to gain better funding and improved relationships with their funders, donors, and clients. Likewise, government departments have attempted to adopt marketing principles to position their effort in the market place and to change people's behaviour, whether it be the Inland Revenue Department ("It's our job to be fair") or the Land Transport Authority ("If you drink then drive you're a bloody idiot").

Additionally, the leisure industry, including the tourism sector, has applied marketing principles with varying success. Novarotov and Crompton (2001) used Critical 
Theory to review the conceptualisation of Marketing in the Public Leisure Industry. They found that service marketing as espoused by Kotler (1975), which is based on an open-system model of formal organisations, the concept of voluntary exchange, and self-interest motivation, were not applicable to the leisure industry because leisure services operate in a bureaucratic environment with the status of monopolists with no direct competitors and stable funding, where self-interest motivation conflicts with the code of ethics practised by public administrators.

It is impossible to see how the marketing mix is applicable in its entirety to the above areas. As well, there have been other drivers of change. Increased globalisation of business has added another dimension to marketing, and the notion of customer retention has become increasingly important. Organisations have recognised the importance of customer loyalty as a key determinant of long-term cash flow. It has been estimated that it costs six times more to gain a new customer compared to keeping an existing customer (Liswood, 1987; Reichheld, 1996).

Therefore, there has been a move from transaction marketing, whereby the single one-off transaction is the determinant of success, to relationship marketing where there is an emphasis on the long-term worth of the customer. Hence there are loyalty programmes and other retention schemes.

Marketing management, like many 'applied' disciplines, tends to follow a pragmatic approach founded on an enterprise discourse based on agendas and concerns set by practitioners. This is in contrast to 'pure' areas where research is linear and developed according to an academic agenda (Tranfield and Starkey, 1998). This 
pragmatic approach is not to be confused with that advocated by Rorty (1998) whereby it is postulated that there is no absolute truth, but artificial boundaries set by research programmes. Rather, the marketing approach is based on naïve realism with boundaries set by the practitioners. Consequently it can be seen as functionalist by nature. Such an approach has used the scientific method to gain academic respectability and in doing so has sacrificed the development of theoretical constructs, as noted by Arndt over 20 years ago. 'Research in marketing gives the impression of being based on a conceptually sterile and unimaginative positivism' (Arndt, 1980 p. 399).

The thesis has selected the marketing management school to explore its philosophical underpinnings due to its dominant position and also its diverse topics of interest. Another reason is that the consumer behaviour school is seen as a separate academic activity, outside of marketing. This issue will be explored in more detail in Chapter Three.

\section{Art or Science}

One of the perennial questions in marketing is whether the discipline should be seen as an art or a science (Kotler, 2005; Brown, 1996). The question can be asked as to how the adoption of the linguistic turn in modern philosophy will affect this debate. It needs to be clarified that the debate is about the best research approach that the marketing discipline should adopt to generate marketing knowledge, and not about how marketing practitioners use knowledge to make decisions (although this is a question addressed in the Exemplary Case - Chapter Seven). To ask whether the marketing discipline is an art or a science begs the question as to what counts as a 
'science' or an 'art'? Unfortunately the marketing literature has concentrated on the former issue, without any in-depth discussion about how marketing could be perceived as an art.

Buzzell defined science as '.. a classified and systematized body of knowledge... organised around one or more central theories and a number of general principles...usually expressed in quantitative terms...knowledge which permits the prediction and, under some circumstances, the control of future events' (Buzzell, 1963 p. 33). However, whilst there has been a search for general theories in marketing over the last forty years, there has been little progress in theory development, as will be discussed in a later section of this chapter. Suffice to say, however, it would seem impossible for marketing to meet the criteria as set out by Buzzell. Hunt (1983), on the other hand, believes that Buzzell's definition is too restrictive. For Hunt, marketing could be called a science when it had a distinct subject matter with underlying uniformities and certifiable research procedures, but at this stage he thought it was too early to make a claim that marketing was a science.

Brown (1996) summarises the debate over the last fifty years. The first stage he calls the pro-science era, which spans from approximately 1945 to 1983 . Here the research approach was based on what could loosely be called logical empiricism, whereby the natural sciences were seen as the model to be adopted. This era was challenged from 1983 to 1999 by the pro-sciences era, where it was postulated, following on from the work of Kuhn (1962) and Feyerabend (1988), science needed to be viewed as nothing more than societal consensus. This relativist approach was attached by Hunt (1983) in particular, who believed that marketing should be based 
on scientific realism. The third stage, from 2000 onwards, is that of anti-science, where the scientific paradigm has been challenged by postmodernism.

Brown (1996) goes on to note five key dimensions that separate scientific from artistic approaches to academic research, as per the following table.

Table $1 \quad$ Key differences between Scientific and Artistic Research

\begin{tabular}{|c|c|c|}
\hline Dimension & Science & Art \\
\hline $\begin{array}{l}\text { Mode of } \\
\text { representation }\end{array}$ & literal & poetic \\
\hline $\begin{array}{l}\text { Criteria for } \\
\text { appraisal }\end{array}$ & validity & believability \\
\hline Generalisations & $\begin{array}{l}\text { extrapolated from } \\
\text { sample }\end{array}$ & $\begin{array}{l}\text { based on the } \\
\text { particular }\end{array}$ \\
\hline Importance of form & $\begin{array}{l}\text { neutral results and } \\
\text { a standard format }\end{array}$ & $\begin{array}{l}\text { form and content } \\
\text { interact }\end{array}$ \\
\hline Ultimate aims & discovery of truth & $\begin{array}{l}\text { creation of } \\
\text { meaning }\end{array}$ \\
\hline
\end{tabular}

Source: Adapted from Brown 1996

From the above table it can be seen that for marketing to be an art the task of the academic changes in terms of the approach taken to generate marketing knowledge. 'If marketing is seen as an art then the marketing academic's role might consequently change from that of researcher to that of critic, connoisseur and communicator and the marketing academic may increasingly turn to the humanities for role models and comparison' (Kavanagh, 1994 p. 34). 
Therefore, is it possible for marketing to be seen as only an art, or is it a blend of both art and science, or should the scientific approach be the sole source of marketing knowledge? This question will be addressed in the thesis and a conclusion to the issue outlined in the final chapter.

\section{Academic Research and Practitioner Relevance}

Another important issue is the divide between research and practitioner relevance, so how will the adoption of the linguistic turn in modern philosophy affect this issue? There are two schools of thought regarding this problem. At one extreme is the view promulgated by Hirschman (1993) who believes that the traditional connection with marketing practitioners is costing the discipline academic objectivity so it should be abandoned. Holbrook (1985) is another author who argues the marketing discipline needs to isolate itself from the practitioner. For him the drive is to understand phenomena for the sake of it, rather than to satisfy some criteria based on the usefulness for practitioners. Hackley (2001) is also of the view that the current academic bias towards managerialism is misplaced. 'Presenting marketing as a value neutral, ahistorical kind of managerial technique is very popular, conceptually simple, ideologically seductive, intellectually worthless, educationally damaging, practically naïve and, ultimately, pedagogically paralysing' (Hackley, 2001 p. 1195).

However, another view is that the role of academic research is to help marketing managers make better decisions. For Jacoby (1985), in contrast to Holbrook, the research orientation should be complementary. Other authors in this group see marketing as an applied discipline and consequently take a managerial perspective. 
For them marketing is an activity so marketing studies are about what marketers do, and the effects of their actions. Brown (1996), on commenting upon the proscientists view that mixing with practitioners has tainted the bid for marketing to gain academic respectability, believes that marketing is an activity based discipline. 'Abandoning the connection with practitioners is the thanatic equivalent of academic educationalists attempting to cut themselves off from educators, medical researchers from practitioners of medicine, legal studies from lawyers, the architectural academy from architects, and scholars of nursing from nurses. The very term "marketing" carries connotations of "doing” (Brown, 1996 p. 266). As Wensley (2002) has noted, marketing as a discipline in most universities resides in a business school or similar type of faculty. The majority of marketing studies takes a managerial focus, with the emphasis being marketing management.

Following on from this, Arndt (1980) believes that there are three different meanings to the word 'marketing'. The first is marketing management which is the applied side of marketing with an emphasis on problem solving. The second is marketing science is concerned with marketing as a social process. The third is marketing ideology which has the task of exploring marketing's underpinnings in the capitalist world. The three meanings are inter-related but Arndt (1980) argues that the three meanings have drifted over the last 20 years, but the major concern of marketing academics has been marketing management (Baker, 2000). Notwithstanding the above, the role of marketing in society needs to be put into perspective. 'Clearly marketing, both as an academic discourse and a management practice, was a response to problems regarding the market and consumption with which 20thcentury business practitioners have been confronted' (Knights 1992 p. 522). Saren 
(2000) also suggests that marketing academics need to critique marketing and its influence on consumer society.

The claim that any critique of marketing belongs outside the discipline does not mean that marketing academics should not take into account the wider community, not just practitioners, in their research programmes. As Wensley (2002) notes in relation to the recent anti-globalisation demonstrations, 'In a very real sense, they are about significant challenges to the primacy of consumption and its systematic effects in our marketing-dominated world' (Wensley, 2002 p. 395).

The question then arises that if one takes a managerial perspective, why is there a divide between academics and practitioners? To answer this question it needs to be remembered that each group has different needs, motivations and reward systems. It is interesting to note that Baker \& Holt (2004) believe that managers found marketing departments in their organisation lacked accountability. They ask the question whether it is the teachers, academics, or consultants who are at fault in not making marketing accountable, and whether academics have concentrated too much on generalizability at the cost of specificity.

Ottensen (2004) states three reasons why practitioners do not use marketing academic literature. First, they see academic articles as useless and trivial. In many cases the information is seen as too complex. Second, they may not have the required knowledge to understand the literature. In many cases, the article needs to be translated by an academic into practice friendly literature but do they have the skills to do this? Finally, practitioners are unaware of the information as the journals 
are not disseminated adequately. A corollary to this is that managers are pressurised with regards to the allocation of time, so the reading of academic articles becomes a low priority.

To confound the issue further, practitioners also conduct marketing research but such research is action specific. It is an attempt to gain answers to specific questions, which in turn are linked to specific problems, and as such the task is not about adding to the pool of academic marketing knowledge. According to Cornelissen, (2002) practitioners build their own theories-in-use based on specific information and tempered by their own experience.

It would seem that the importance of relevance is dependent on cultural context as American marketing academics view the role of theory differently from European academics '...this supports the conclusion that Americans tend to view marketing theory as something that belongs in the classroom under the control of academics for academics, while the Europeans tend to see marketing theory as something that is helpful to marketing practitioners dealing with the real world' (Howard, Savins, Howell, \& Ryans, 1991 p. 13).

Given the brief synopsis of the relationship between academic research and practitioner relevance the question arises whether a new paradigm will address this problem. Schön in the 'Reflective Practitioner' (1983) is interested in the way practitioners undertake decision making. He believes that 'technical-rationality', that is the application of scientific theories to instrumental problems, is based on a positivist epistemology and as such, has failed to resolve the dilemma between rigour 
versus relevance that is faced by practitioners. Schön believes that technical-

rationality does not take into account the uniqueness of the situation.

'The dilemma of rigour or relevance may be resolved if we can develop an epistemology of practice which places technical problem solving within a broader context of reflective inquiry, shows how reflection-in-action may be rigorous in its own right, and links the art of practice in uncertainty and uniqueness to the scientist's art of research. We may thereby increase the legitimacy of reflection-in-action and encourage its broader, deeper and more rigorous use' (Schön, 1983 p. 69).

He makes a distinction between reflection-in-action and reflection-on-action. The

former involves taking account of previous experiences, connecting with our

intuition and utilising our theories in use. From this process new understanding is

gained to influence the managers' actions.

'The practitioner allows himself to experience surprise, puzzlement, or confusion in a situation which he finds uncertain or unique. He reflects on the phenomenon before him, and on the prior understandings which have been implicit in his behaviour. He carries out an experiment which serves to generate both a new understanding of the phenomenon and a change in the situation' (Schön, 1983 p. 68).

Schön believes it is not a matter of following a set practice, outlined in a textbook, as every case is unique. It would seem that Schön is drawing upon Artistotle's distinction between phronesis and the practical.

Reflection-on-action is done after the decision has been made, and occurs often when talking with co-workers and supervisors. This is where reflective thought occurs and allows the practitioner to build up a repertoire of images, ideas and examples.

'In this way we engage with a situation. We do not have a full understanding of things before we act, but, hopefully, we can avoid major problems while 'testing the water'. When looking at a situation we are influenced by, and use, what has gone before, what might come, our repertoire, and our frame of reference. We are able to draw upon certain routines. As we work we can 
bring fragments of memories into play and begin to build theories and responses that fit the new situation' (Schön, 1983 p. 70).

It would seem that reflective practice entails praxis. It involves the practitioner reframing the situation to allow for creative practice. However, it is not being suggested that theory is abandoned for practice. Rather, it is a matter of fusing the two so that praxis is developed, thus allowing practitioners to develop in a professional manner. Therefore, the thesis will explore the development of praxis within the postmodern context and determine how the linguistic turn in modern philosophy will affect the academic-practitioner divide.

\section{Theory}

This section explores the issue of theory development for the discipline of marketing management, as a number of authors have claimed that this is an issue for marketing (Brownlie \& Saren, 1992; Razzaque, 1998; Saren, 2000; Burton, 2005). Therefore, what will be the effect of a new approach to marketing based on the linguistic turn in modern philosophy on theory development? However, before this question can answered in Chapter 8, at this stage it is important to understand the notion of theory and theory development, from a logical empiricist point of view.

The logical empiricist argue that the art of theory-building is important as it provides both academics and practitioners with a meta-language, which allows for the clarification of ideas, assumptions, and rules of thumb used in making day to day marketing decisions. An expertise in theory-building allows the practitioner to more quickly understand the world in which they work, enables better predictions, as well 
as embrace new tasks that have increased responsibility. 'The meta-language of theory construction is common to all contexts since it is a way of thinking about thinking' (Zaltman, Lamasters \& Heffring, 1982 p. 20).

However, the problem with the word 'theory' is that is has many different meanings. 'Like so many words that are bandied about, the word theory threatens to become meaningless. Because it referents are so diverse - including everything from minor working hypotheses, through comprehensive but vague and unordered speculations, to axiomatic systems of thought - use of the word often obscures rather than creates understanding' (Sutton and Staw, 1995 p. 371).

Although the word is often used in different contexts with varying meanings, it is worthwhile to define the term 'theory'. The Concise Oxford Dictionary defines theory as 'a supposition or system of ideas explaining something, esp. one based on general principles independent of the particular things to be explained' Thompson, 1995 p. 1446). Given this definition it is important to note that a theory is explaining independent variables. In logical empiricist type of work this relationship is represented by a theoretical framework (O’Leary, 2004).

However, as noted by Sutton and Staw (1995), frameworks in themselves are not theories. What is necessary is for causal links to be shown. Based on the correspondence theory of reality, logical empiricism uses hypotheses to bridge the gap between theory and reality, represented by data. ${ }^{2}$ The difference between theory

\footnotetext{
2 Note: Problems with logical empiricism and the correspondence theory of reality will be discussed in detail in Chapters 3, 4, and 5.
} 
and hypotheses (Baumol, 1957) is that a hypothesis is a question of fact, whereas a theory is an explanation in a systematic manner.

Prediction is a basic tenet of theory (Peter, \& Olson, 1983), so when a theory fails to predict adequately, the theory is brought into question. In this respect a theory is an attempt to answer the question 'why?' 'Theory emphasizes the nature of causal relationships, identifying what comes first as well as the timing of such events. Strong theory, in our view, delves into underlying processes so as to understand the systematic reasons for a particular occurrence or nonoccurrence' (Sutton and Staw, 1995 p. 378).

Therefore, the task of the theorist is to obtain a systematic explanation. Why is theory necessary? Unfortunately, facts do not speak for themselves. It is possible to obtain correlations from different facts but that alone does not provide structured relationships. The task of the theory builder is to obtain a simplified model, which tends to approximate the facts rather than merely describe them.

What counts as good theory? According to Baumol (1957) there are three characteristics of a good theory. The first is that a good theory needs to be simple so that systematic manipulation and analysis can be undertaken to confirm the theory. The second characteristic is that the theory must be a close approximation to the relevant facts. Finally, a good theory must have conclusions that are relatively insensitive to any changes in the theory's assumptions. 
According to Skipper \& Hyman (1990), to develop theory marketing must adopt an argument-centred style of work which relies on words and sentences that fit a framework with premises that lead to a conclusion. Such arguments used to explore the relationship between concepts and theories rely on conceptual analysis and not statistics or arithmetic and so forth. Such an approach is philosophical by nature. 'Marketing scholars, with every theory piece and every think piece they write, are doing philosophy, whether they know it or not. As long as they are doing philosophy, their own intellectual honesty should be driving them either to learn how to philosophize well or to collaborate with trained philosophers' (Skipper \& Hyman, 1990 p. 90).

Nonetheless, it needs to be acknowledged that a theory is developed in a social setting, as a theory reflects the social and historical circumstances at the time it was developed. According to Murray, Evers \& Janda (1995), a theory is made up of superstructure and a substructure. The superstructure involves a set of propositions which may be empirical, interpretive or critical in form. As such they can be analysed by the researcher. On the other hand, the substructure consists of the values, interests, and assumptions of the researcher and is the guiding influence on the superstructure. Substructures are not easily identified but can be inferred with careful analysis.

The socialization process plays a big part in the development of substructures. An important input into this process is the $\mathrm{PhD}$ programmes undertaken by new academics because such programmes set the agenda and also develop the competence for future research. In addition to socialization and cultural diversity 
aspects, the social context also plays a part in the development of substructure. This includes the purposes of the researcher and any significant social changes that occur over time.

How well has marketing fared with theory development over the last fifty years? Over forty years ago Buzzell (1963) wrote that whilst there was a substantial amount of classified marketing knowledge no central theory had been developed. Also there were few accepted principles, and any research results obtained did not allow for future predictions in marketing to be made with any certainty. Leone and Schultz (1980) attempted to capture marketing knowledge but could not find many scientific findings. Whilst they found a great deal of empirical research, most of it was one-off studies without any generalizability. Howard et al (1991) found that there was a general agreement between both American and European academics that there was a paucity of work being done by academics on marketing theory, especially compared with other social sciences. As Gummesson (2001) notes, there are a small number of specific marketing theories with limited scope of application. 'Marketing management is currently a patchwork of fragmented models, assumptions, case stories and checklists on top of a partially obsolete foundation: micro-economics, marketing mix, four Ps, and the marketing of packaged consumer goods' (Gummesson, 2001 p. 29).

Given that marketing to date has been poor in theory development, what is stifling theory development in marketing? According to Hunt (1994), two forces influence the research undertaken in marketing. One is the interests and abilities of marketing academics, and the second is requirements and biases of journal editors and 
reviewers. With the latter group, there is an implied requirement for any claims made in articles to be substantiated with reference to other articles, thereby rejecting any original ideas. Hunt also believes that a lot of the work that is cited to substantiate claims comes from outside the discipline. He sees marketing as an applied discipline, meaning that what is 'applied' are theories borrowed from other disciplines. Whilst theory borrowing is rife in marketing bringing inherent problems, it is an unusual use of the term 'applied'. A more common meaning refers to the fact that marketing is concerned with practical expertise of marketers in the broad business arena. In positivist terms, what is of concern is the relationship between theory and practice in a managerial context.

It would seem that one problem in generating marketing theory is that research is undertaken at a micro-level without any absorption into a larger understanding. 'Too often in marketing we undertake research projects to investigate the relationships between isolated variables and we do not make an attempt to integrate our findings into an overall framework' (Carson \& Coviello, 1996 p. 56). November (2004) uses the analogy of the brick wall, where marketing academics are deemed to be very good at making separate bricks but they are unable to build a wall.

Regrettably the marketing discipline is seen by other academics as being theoretically weak and having not much to offer, as marketing borrows theories from other disciplines. Unfortunately the reverse is not true, that is, not many citations of marketing theory are made by other disciplines (Willmott, 1999). According to some authors the above weakness is compounded by the fact that the marketing discipline has clung to an outmoded philosophy of science. 
'There has been a revolution in the philosophy of science literature in the past twenty years or so. While there are still those who cling to and attempt to shore up weaknesses in the traditional view of science, much of the philosophy of science literature involves approaches which bear little resemblance to logical empiricism. While perhaps not recognised as such, logical empiricism is the dominant philosophical approach employed in marketing and it has come to us in our borrowing of theory construction and research methods from psychology and economics' (Peter, 1982 p. 11).

Theory borrowing in marketing comes from areas such as economics, organisational behaviour, psychology and sociology. Over and above the gatekeeper roles within the discipline as outlined above by Hunt, theory borrowing is very tempting especially with a young discipline as it is a good source for new ideas (Arndt, 1985). Murray et al (1995) also outlines further reasons why theories are borrowed in marketing.

'Theories are borrowed in marketing for a variety of purposes. First, theories constructed in other fields are sometimes tested within the domain of marketing. These types of tests are often done to explore the boundaries of a given theory. For example, the generalizability of the elaboration likelihood model (Petty \& Cacioppo 1980, 1981), was enhanced when it was shown that it applied, not only in situations where people were being persuaded on political and social issues, but also where people were being persuaded on purchase-related issues (Petty, Cacioppo, and Shuman 1983). Second, researchers can use theory borrowing to solve a particular applied problem. For example, the elaboration likelihood model might be used to develop a better advertisement for a low-involvement product. Finally, a theory may be borrowed to enhance understanding of marketing phenomena. This purpose occurs more within the context of discovery than justification' (Murray et al, 1995 p. 96).

Despite the attractions there are some pitfalls in theory borrowing. Whilst this is one way to grow a discipline it is easy to fall into the trap of applying theories indiscriminately without any thought to the applicability to the overall discipline. This in turn, can lead to opportunistic behaviour. An outcome of such approaches leads to marginalisation of the discipline due to research containing mundane and 
trivial findings (Razzaque, 1998). 'Replicability is a mere technical definition of objectivity that does in no way assure the scientificity of the knowledge generated' (Razzaque, 1998 p. 7).

Another danger of theory borrowing from another discipline is that it can be used out of context. Often the theory will be applied to explain different social phenomena but because the context changes, so does the meaning. An example used by Murray et al (1995) is the borrowing from biology of population ecology. This theory concerns the adaptation of a species to the physical environment. The environment is seen as stable and ordered with agents adhering to a strict script. But the theory does not fit easily into the marketing context as it ignores the decision making of the agents where conscious choice is an integral part of the environment.

Critical reflection is needed when borrowing a theory to make sure there is consistency between the original context and the new context. 'In order to consider the full implications of borrowing, the propositions, underlying cognitive interests, and key contextual influences need to be understood. Since the substructure is often hidden, this process involves reflection' (Murray et al, 1995 p. 101). The first step is to interpret the elements of the structure of the theory and understand it in its original context. The next step is to determine what elements would change when the theory is applied to the new field. Here the task is to look for contradictory patterns. If inconsistencies are found it does not mean that the theory cannot be borrowed but rather, assuming a researcher is aware of the problems, care can be taken with the implementation of the theory. 
Notwithstanding the above, theory borrowing in marketing has been indiscriminate and at times, opportunistic, with the result that results become trivial and the marketing discipline becomes marginalised (Razzaque, 1998).

This section has outlined the topic of theory from a logical empiricist point of view. The relationship between theory and hypotheses has been explained and problems associated with theory development within the discipline have been examined, in particular the problems associated with theory borrowing. It is now necessary to explore in more detail the issue of the linguistic turn in modern philosophy and how it affects the generation of marketing knowledge. Once this has been undertaken, the final chapter will return to address the issue of theory development within the marketing discipline.

\section{Structure of this Thesis}

The structure of the thesis is as follows: the next chapter begins with a discussion on Thomas Kuhn's paradigm concept as he was the first person to popularise the concept by applying it to the philosophy of science outlined in his book 'The Structure of Scientific Revolutions' (1962). What he is saying is that a paradigm delineates the key concepts and rules to be followed within a given scientific endeavour. He believes that disciplines change in terms of the key reference points by undergoing a scientific revolution. This means that the disciplines shifts from a normal to a revolutionary science. Burrell and Morgan (1979) have utilised the paradigm concept and developed a framework to describe different research approaches in the area of organisational studies. Arndt (1980) has adopted the 
framework and applied it to the discipline of marketing. However, can sense be made of competing paradigms?

Kuhn argues that paradigms are incommensurable, that is, observations and understandings in one paradigm cannot be meaningfully translated into another paradigm because their basic assumptions cannot be reconciled. However, this is not to say that paradigms cannot be compared and contrasted at an ontological, epistemological and methodological level. What cannot be claimed is that one can evaluate a number of different paradigms and pronounce that one and one only paradigm is the 'right' one. According to Burrell and Morgan each paradigm has ontological, epistemological and methodological constructs that differentiate one from another. However, it will be argued that the Deetz framework should be adopted as it represents the linguistic turn in modern philosophy.

Chapter Three explores the dominant paradigm in marketing, logical empiricism, also known in a general sense as positivism. Such an approach is linked to the socalled scientific method. There are problems with this approach, namely: Frameworks and theories have been borrowed from other disciplines, such as psychology, economics, and sociology which have hampered any attempt at original theory development. Measurement and analysis have been the primary focus, which has meant an over reliance on statistics and quantitative analysis. As noted by Willmott (1999) this is due to a desire to gain academic status by emulating the natural sciences. Consequently much of the marketing literature is informationincreasing but not knowledge-extending. 
Hunt argued that scientific realism should be adopted by marketing as their philosophy of science. This has been challenged by Anderson and others who claim that an interpretivist approach should be used to generate marketing knowledge. Such viewpoints are inadequate as they adopt a foundational view of knowledge.

Finally, a Lakatos framework was applied to the marketing management literature and it is assessed as falling short of Lakatos' criteria to be decreed as a progressive discipline.

The next chapter explores postmodernism as any discussion of the philosophy of science pertaining to marketing needs to take into account this phenomena as it challenges the basic tenets of modernism, namely that there is progress in thought and consequently we learn from the past. Postmodernism also challenges the search for universal theories and the notion that science is value neutral.

Postmodernism provides a framework to understand the dramatic changes taking place in the marketing arena and challenges some of the traditional marketing principles. Furthermore, postmodernism disputes the epistemological foundations of the dominant paradigm of the marketing discipline.

Postmodernism can be viewed from two vantage points. The first is to view it as a cultural analysis and the second is to view it from a philosophical perspective. These two constructs can be understood from two further distinct vantage points, namely an affirmative postmodernist and a strong postmodernist. Four distinct quadrants are explored by analyzing the work of selected authors. Cultural analysis from an 
affirmative postmodernist is seen through the eyes of Jamieson (1984). In comparison to his views, the work of Baudrillard $(1978,1979, \& 1983)$ is explored as he is seen as strong postmodernist. The strong postmodernist philosophical point of view is explained through the eyes of Foucault $(1967,1972,1974, \& 1982)$ and Derrida $(1982,1988)$.

In terms of postmodernism as a cultural analysis both Jameson and Baudrillard offer some insights but from a marketing perspective much of the postmodern cultural analysis comes as no surprise. However, in terms of the aims of this thesis, the philosophical quadrants are of more interest. Both Derrida $(1982,1988)$ and Foucault $(1967,1972,1974, \& 1982)$ have been discussed and in general terms have been found wanting for a number of reasons, but primarily because of their pessimistic outlook. The problem for postmodernism is that by retreating into a culturalist and theory-laden discourse all that is possible is deconstruction of social problems with the result that this is no different from Freyerabend's 'anything goes' position, one that ultimately affirms the existing social order (Delanty, 1997). As a generalisation of their relative positions it would seem that they do not offer any remedy to the problems they present. According to Brown, (1993) it would seem that demolition is the only construction that postmodernism recognises

Chapter Five explores neo-pragmatism with particular reference to the work of Richard Rorty $(1979,1982,1989)$. This philosophical approach can be seen as falling into the fourth quadrant as per the framework outlined in the previous chapter. In other words, it can be seen as taking an affirmative postmodern stance from a philosophical position. 
Pragmatism could be classed as one of the most misunderstood terms within philosophy. Unlike analytical philosophy, it is not based on theoretical notions or about discovering truth. Instead, it is about a way of doing philosophy. What this means is that, at a basic level, what is considered is a course of action and its observable consequences. Furthermore, it is the sum of these consequences that forms the meaning of the action. Therefore, pragmatism is a method for evaluating philosophical problems by working through the practical consequences. This means that theory and action are intertwined, each modifying the other whilst at the same time maintaining there mutual relevance.

Richard Rorty has brought pragmatism into the limelight but other major philosophers have also given credence to the school of thought, namely, Hilary Putnam (1997) and Richard Bernstein (1983). Rorty claims that pragmatism blurs the distinction between objective and subjective as well as fact and value. He leaves room for alternative narratives and promotes intellectual tolerance.

Pragmatism is about a way of doing philosophy. It weaves together theory and action to determine beliefs that clarify meaning and allow action to be seen as applicable for a given situation in a given context. Pragmatism gives rise to the adoption of hermeneutics as ontology and leads into narrative theory.

Chapter Six explores narrative theory as a way to generate marketing knowledge. What is of interest is how language is used to construct meaning. How do marketing managers use stories to give direction to actions within the workplace? 
Narrative theory provides theorists and practitioners with an additional lens to understand marketing management. The term narrative is used to refer to thematic, sequenced interpretations that convey a meaning created by an author for a reader. Story is a type of sensemaking utilised by marketing managers to understand their actions. Narrative highlights the discursive, social nature of marketing management within a cultural and historical context.

Marketing managers use stories to persuade people to understand or to carry out a certain action. In this sense, the authors of the story need to tell a compelling account that others are prepared to buy into and implement. The story that the author selects to tell is one of many possible plots and scenarios. By understanding the stories that are told it is hoped that such interpretations allow new insights and trains of thoughts on the subject matter rather than a testable truth.

Chapter Seven is a case study to exemplify how neo-pragmatism can be used in a marketing context. Air New Zealand was chosen as the company to study as they had significant marketing management decisions that were made and were implemented over the last three years.

Narrative theory is used to gain an understanding of the issues faced by Air New Zealand marketing management and how they implemented the strategy to obtain 'buy-in' from customers. Also important was how they attempted to persuade travel agents to accept the new domestic market proposition as they were still reliant on their services for selling international tickets. 
The final chapter brings together the argument of the thesis and shows how a new research approach can address the problems outlined above, namely the concern surrounding marketing's status as an art or a science; the widening gap between academic research and practitioner relevance; and the lack of theory development in the marketing domain.

\section{Methodology}

The thesis will use a philosophy of science lens to view the generation of knowledge within the marketing arena. Therefore, the methodology is to critique marketing's philosophical foundations and to suggest an alternative research programme by utilising the history of philosophy, with particular emphasis on the philosophy of social science, combined with conceptual analysis. The operative word is 'lens', as the thesis is not a philosophical thesis as such, but utilises reflective discourse and argument to explore marketing's philosophical foundations. The thesis will also draw on literature from related disciplines, for example management studies, to provide a comprehensive perspective as, it is argued, the marketing management discipline has been slow to grasp the trends in postmodern literature.

The philosophy of science lens will be based on three important dimensions. The first dimension is a review and adoption of a paradigmatic framework. With this respect the work of Kuhn (1962), Lakatos (1970), Burrell and Morgan (1979), Arndt (1983) and Deetz (1996) will be reviewed. The second dimension is the linguistic turn in modern philosophy, linked with postmodernism. In particular the work of Jameson (1984), Baudrillard (1978, 1979, 1983, 1988, 1994), Derrida (1988), 
Foucault $(1967,1972,1974)$ and Rorty $(1979,1982,1989)$, will be examined.

Finally, the third dimension, that of praxis, based on Aristotle's concept of phronesis and developed by Gadamer (1975), will be explored.

\section{Conclusion}

The thesis argues that marketing management should adopt the linguistic turn in modern philosophy. The marketing management school was selected for analysis as it is the dominant school and consumer behaviour is seen as a separate discipline. The thesis uses a philosophy of science lens to explore the philosophical underpinnings of the marketing management school. It then explores the linguistic turn in modern philosophy, represented by postmodernism, and explores an exemplary case to show the application of a postmodern research protocol on a marketing management problem.

Three issues were identified as being important to the discipline, namely art versus science; theory development; and relevance, and the task is to determine the effect that the linguistic turn in modern philosophy will have on these issues. 


\section{Chapter 2: Beyond Paradigm Wars}

\section{Introduction}

In Chapter One it was argued that marketing should adopt the linguistic turn in modern philosophy. To justify this argument the philosophical underpinnings of marketing management need to be explored. A good place to start such an exploration is the work of Kuhn (1962) in terms of his work on scientific revolutions. This will be followed by other work regarding the use of the concept of paradigms, firstly by Burrell and Morgan, in relation to their framework published in 'Sociological Paradigms and Organisational Analysis'and secondly by Johan Arndt (1985) in his work on Marketing Theory. After a discussion regarding the benefits and problems of such frameworks, an alternative framework developed by Deetz (1996) is introduced. This framework is based on the use of discourse in research approaches and provides a good framework to explore different research programmes. The notion of incommensurability will then be explored. Central to the problem of incommensurability is the concern surrounding the evaluation of different paradigms. Finally, the role of different scientific research styles is explored. This chapter will then allow an in-depth discussion in Chapter Three of the dominant paradigm in marketing in contrast to interpretivism.

A number of authors have recently made the claim that a new marketing paradigm is needed. Gronroos (1994) states that a 'paradigm shift in marketing is needed if marketing is going to survive as a discipline.' Other authors, such as Buttle (1994) and Brodie (1997) also reiterate the claim that relationship marketing is a new paradigm. However, the debate is not new, as can be seen with reference to Johan 
Arndt's (1985) paper in the Journal of Marketing, and the debate between Hunt and Anderson, which is covered well by Kavanagh (1994).

What paradigms are the contenders for the new marketing? Stephen Brown (1993, 1994, and 1999) has written extensively about post-modernism. Hackley (1998) has written about the use of the social constructionist perspective for qualitative research in marketing. Burton (2001) has shown how critical theory can be used in marketing discourse, and recently Easton (2002) has introduced critical realism as a contender for the marketing science school.

But before a new marketing paradigm can be explored, it is necessary to explore some issues. To begin, it is necessary to understand what is meant by the term 'paradigm'. Also the distinctive ways in which research is undertaken needs to be explored. It is not only a question concerning the methodology that is used in any given research programme but also the assumptions that are behind the methodology. When comparing different research programmes by undertaking reflective discourse the basic requirement is to look for consistency and coherence, and to explore the ontological, epistemological, and methodological premises that have been employed. 


\section{Kuhn}

An appropriate place to examine the concept of paradigm is the work of Thomas

Kuhn (1962), who was instrumental in introducing the concept to the wider academic community. Kuhn's argument is concerned with the development of a unified body of science from a historical point of view.

To describe the different ways of conducting science Kuhn used the term 'paradigm'. Scientific knowledge can be examined and explored within a paradigm, which has its own criteria of scholarliness upheld by its own school of researchers. A paradigm delineates the key concepts and rules to be followed within a given scientific endeavour and also plays a role in guiding observation and experiment. Such ways of conducting scientific research influence the questions that are asked just as much as the answers that are given. Truth, therefore, is a matter of consensus rather than a correspondence with reality as the Positivists would suggest. ${ }^{3}$

Kuhn believed that science was not conducted inductively, from observation to theory, or by falsification, as Popper (1959) had thought, but that science shifted from what he called normal science to revolutionary science. 'In this essay, 'normal science' means research firmly based upon one or more past scientific achievements, achievements that some particular scientific community acknowledges for a time as supplying the foundation for its further practice' (Kuhn, 1962 p. 10). The shift begins when, in the process of conducting normal science, doubts about the assumptions and procedures creep in leading to ad hoc modifications being made.

\footnotetext{
3 Problems with Positivism and the Correspondence Theory of Reality will be discussed in detail in Chapter Three.
} 
According to Kuhn, theories that fit into existing paradigms become part of the extant knowledge. However, those that don't fit are rejected, often with vehemence.

But it would seem that one reason such theories are rejected is not because of the content of the theory but how the theory was derived, that is, the process or methodology. A crisis develops in the normal science when an anomaly is regarded as particularly serious because it strikes at the fundamentals of a paradigm and at the same time persistently rejects attempts by the members of the normal scientific community to remove it. Anomalies are also regarded as serious if they are important with respect to some pressing social need. The number of serious anomalies is a further factor influencing the onset of a crisis. If the modifications are not sufficient to quell the doubts and anomalies, a scientific revolution will develop. A struggle takes place whereby new theory is mediated by the anomalies of the normal science. A rival paradigm will regard different kinds of questions as legitimate or meaningful, and it will also involve different and incompatible standards compared to the normal science.

'All crises begin with the blurring of a paradigm and the consequent loosening of the rules for normal research. ... Or finally, the case that will most concern us here, a crisis may end with the emergence of a new candidate for paradigm and with the ensuing battle over its acceptance' (Kuhn, 1962 p. 84).

This revolution involves a change in terms of the key reference points and can arise due to either intellectual or institutional factors. The former is when the set of guiding principles change because of unexpected results in the normal science. The latter occurs when the wider social mechanisms in which the normal science exists change, therefore affecting the way the normal science is regulated. In other words, the distribution of power in the wider society that keeps the normal science on track changes, thereby forcing a scientific revolution. 
Kuhn observed that the majority of scientists stay within the dominant paradigm as it offers them security. Paradigms have a persuasive influence on science practice, and therefore can only be replaced by a revolutionary process. 'Almost always the men who achieve these fundamental inventions of a new paradigm have been either very young or very new to the field whose paradigm they change' (Kuhn, 1962 p. 90).

According to Phillips (1987), the revolutionary metaphor used by Kuhn is seen as an exaggeration as change within disciplines has often been found to be gradual. Kuhn is explicit how over time there can be divergent programmes operating, with different approaches to scientific problem-solving. However, they are resolved on a temporal dimension because over time, albeit the pace can be slow, researchers depart the 'normal' science and its practices and adopt the revolutionary approach. For a paradigm shift to take place it often requires a new generation of scientists, so the cultural values of the research programme change. Kuhn's study of disciplines in the natural sciences has shown that a single paradigm has eventually dominated. A mature science is governed by a single paradigm, which sets the standards for what is considered to be legitimate work.

'Copernicanism made few converts for almost a century after Copernicus' death. Newton's work was not generally accepted, particularly on the Continent, for more than half a century after the Principia appeared. Priestly never accepted the oxygen theory, nor Lord Kelvin the electromagnetic theory and so on. The difficulties of conversion have often been noted by the scientists themselves. Darwin, in a particularly perceptive passage at the end of his Origin Of Species, wrote: "Although I am fully convinced of the truth of the views given in this volume ... I, by no means expect to convince experienced naturalists whose minds are stocked with a multitude of facts all viewed, during a long course of years, from a point of view directly opposite to mine. ... But I look with confidence to the future - to young and rising naturalists, who will be able to view both sides of the question with impartiality." And Max Planck, surveying his own career in his Scientific Autobiography, sadly remarked that "a new scientific truth does not triumph 
by convincing its opponents and making them see the light, but rather because its opponents eventually die, and a new generation grows up that is familiar with it' (Kuhn, 1962 p. 150).

One criticism levelled at Kuhn by Hutchinson (1976) was that he was too concerned with the development of physics, particularly the scientific story of its development from the sixteenth century onwards. According to Hutchinson, his approach can be viewed as oversimplified historicism, which leads to how science should develop.

Therefore, is Kuhn giving us a descriptive account about scientific activities or a prescriptive account about how science should proceed? But this may not be central to the issue. What is central to the thesis is that there are different paradigms in the social science, which in turn affect our view of the world.

Kuhn characterised science as a social enterprise, carried out by people with beliefs, needs and ambitions, who are part of a scientific community. Any changes in a scientific practice are not only rational but also social-psychological, often involving great leaps of faith. According to Phillips, (1987) Kuhn challenged the traditional view that science was a continuous process, undertaken by a series of tests. Instead, Kuhn believed science was discontinuous in the sense that it operated within a distinct paradigm. Therefore, each paradigm had its own set of rules and criteria for judging the merits of a piece of work. These key concepts and methodological rules are within a paradigm. Consequently, scientific results cannot be critiqued by people working within another paradigm. Also, such an approach leads to relativism, because if there are no overall criteria, any approach can be considered to be good as

Deleted: However, prima facie the notion of incommensurability is a circular argument, as a theory
can only be judged by the set of rules within its own paradigm. any other approach. ${ }^{4}$ Kuhn also claimed that it was not rationally possible to move between paradigms. This is because a scientist working in one paradigm knows and 
understands the concepts and rules particular to that paradigm. One could not switch to another paradigm as they have no way of judging the benefits of the new paradigm. What criteria would they use to evaluate the benefits? (Phillips, 1987).

Unfortunately Kuhn uses the word 'paradigm' in twenty-one different senses (Willmott, 1993), so there is a difficulty in obtaining a clear definition of how the term is being used. Nevertheless, Morgan (1980) has grouped the definitions into three main categories. The first is where the term is used to describe a complete view of reality. The second is where paradigm is used to describe differences in the social sciences schools of thought. Finally, the term is used to describe specific use of instruments used in problem-solving. It is the second view that this thesis is attempting to explore. Does the history of the natural sciences in terms of Kuhn's theory development make sense for marketing? His theory assumes that a sense of progress ascribed to the natural science is desirable and that social sciences must follow the same path. But Kuhn saw that the social sciences were fundamentally different from physics. He termed them proto-sciences and saw them as being more like the arts and philosophy as they had different developmental patterns than the established sciences.

'Immature or proto-sciences are those in which practice does generate testable conclusions but which nonetheless resemble philosophy and the arts rather than the established sciences in their developmental patterns ...The proto-sciences like the arts and philosophy, lack some element which, in the mature sciences permits the more obvious forms of progress. It is not, however, anything that a methodological prescription can provide' (Kuhn 1970 p. 244).

So it may be that Kuhn's Scientific Revolutions is not applicable to marketing per se. However, his work on paradigms heralded a new approach to look at scientific research. According to Cannella \& Paetzold (1994), one of the benefits of Kuhn's 
work is that all knowledge is socially constructed. For science to grow and develop there needs to be competing schools of thought. Paradigm is a useful term as it reminds us of the fallacy of evaluating particular theories without understanding the ontological and epistemological assumptions which are the foundations of such theories. His is a positive history of science where he is interested in problemsolving in an accepted theoretical framework. The distinctive feature of his work is the description of 'scientific revolutions' which signal a sharp break in the development of science. However, such revolutions, where there is a mutual incomprehension between scientists during a period of intellectual crisis, need to be seen as a rhetorical exaggeration as the stated difference is a matter of degree. It would seem that such differences emerge only after a long period of intellectual competition (Blaug, 1976).

It would seem that the word paradigm is being used in marketing literature to describe a new body of knowledge. For example, Achrol (1997) talks about the need of a new paradigm to take into account network organisations. The reader is being exhorted to leave the marketing mix (4Ps) behind and adopt a new way of describing marketing. But such an exhortation begs the question as to what method should be used to describe and explore this new 'paradigm'. So we end up with the issue exposed by Burrell and Morgan, which will be explored in the next section.

However, before the start of the next section it is apposite to quote from Wartofsky (1976) about the impact Kuhn had on philosophers and the philosophy of science.

'There is more history to this story, but its happy and serendipitous culmination came when a bona-fide historian, Thomas Kuhn, with an excellent book on Copernicus to his credit, and with the influences of his colleagues - Leonard Nash and Paul Feyerabend, and of Polanyi as well - 
came out with a blockbuster - The Structure of Scientific Revolutions - an irritating, naïve, confused and provocative work, which nevertheless, brought it all together in one glorious explosion.

The sociology of the reception of The Structure of Scientific Revolutions is an interesting subject in its own right, and I won't try to get into it here. But the results are well known. Anti-positivist, anti-reconstructionist history of science had found a naïve but provocative formulation which shook up the philosophers of science. Small wonder. They knew little history - especially history of philosophy - so it all looked new to them. They were incompetent sociologists, so the sociology of science alleged in Kuhn's work simply had to be accepted enthusiastically or rejected violently on a priori philosophical grounds. What the philosophers could do was talk about paradigms (which they did endlessly). But the effect, one way or another, was to turn many of them to the history of science, and to afford a new legitimation to the philosophical reflection on this history, whether in criticism of or in agreement with Kuhn' (Wartofsky, 1976 p. 729).

\section{Burrell and Morgan}

Burrell and Morgan (1979) in 'Sociological Paradigms and Organisational Analysis'

(Paradigms) stress that the scientist's frame of reference plays an important part in the generation of research. They postulate four key paradigms based upon the metatheoretical assumptions based on the nature of social science and the nature of society. Each of these four paradigms has their own ontological and epistemological beliefs, as well as beliefs about human nature. Following this each paradigm has a preferred methodological approach.

Ontology is concerned with the essence of the phenomena being investigated. Is the reality perceived to be external to the investigator and therefore viewed objectively, or is the reality a product of individual consensus so it is viewed subjectively? This distinction, between objective and subjective, is a debate between realism and nominalism. The realist sees the social world as external, consisting of hard, tangible structures, which exist independently. This view would see the marketing world as 
hard and concrete, just like the natural world. On the other hand, for the nominalist the external world is based on individual cognition where names, concepts, and labels are used to structure reality. 'Names' are an artificial creation and are only tools for describing and making sense of the world as it is perceived to exist.

Epistemology is concerned with how we come to know about the world. Is knowledge acquired or experienced? Basically, there are two lines of approach in understanding the problem surrounding the acquisition of knowledge. The first is that of the positivist tradition, which is an umbrella term to mean that the only authentic knowledge is scientific knowledge. Positivism is interested in regularities and causal relationships in the different elements being investigated. It is based on traditional natural sciences and believes that the growth of knowledge is a cumulative process with new insights added as time passes.

Under the general concept of positivism there are two main groups. The first is that of logical positivism, which stemmed from the Vienna Circle $^{5}$ in the 1920 's, led by Moritz Schlick. In Britain the main proponents were A.J. Ayer and Bertrand Russell. The treatise of the logical positivists was applied to all philosophical problems. They wanted to reduce all knowledge claims to truths of logic and mathematics and confirmed with reference to sense experience. The outcome of this approach was the elimination of metaphysics, aesthetics and ethics. Their epistemology was based on the verifiability criterion of meaning with any knowledge statements being empirically verifiable.

\footnotetext{
5 The Vienna Circle was a group of philosophers, led by Moris Schlick, who believed that there was only one source of knowledge and the most appropriate method to solve philosophical problems was the use of symbolic logic. Two prominent philosophers who were members of the group were Rudolf Carnap and Herbert Feigl.
} 
The second development was that of logical empiricism, which was less rigorous than logical positivism and did not have the same broad agenda, so they ignored metaphysics, aesthetics and ethics. They were more concerned about epistemology and how knowledge could be generated. They believed that knowledge begins with observation from which theories are developed and new evidence obtained to either confirm or deny the proposed theory. Therefore, logical empiricists use the hypothetico-deductive method so any truth statements rest on theory and testability. They tend to rely heavily on statistical analysis so any knowledge statement is supported by probabilistic data. Logical empiricists, like the logical positivists, still believe that knowledge is discovered by using scientific procedures, with a goal to establish explanatory arguments based on causal relationships.

The second line of approach to the acquisition of knowledge is the anti-positivists who are not interested in the search for laws as they see the epistemological investigation as being essentially relativistic. It is the point of view of the individuals who are involved in the research process that is of paramount importance.

Methodology issues fall basically into two camps. The nomothetic approach emphasises research which is based on systematic processes and techniques. Consequently, there tends to be a preoccupation with the analysis of data as a key method. The ideographic approach emphasises first-hand knowledge that is obtained from the phenomenon under investigation. Consequently, the approach relies on personal reports and the analysis of subjective accounts. 
Burrell and Morgan's matrix is based on the subjective/objective dichotomy and the nature of society. In terms of the latter dichotomy, Burrell and Morgan take two opposing points of view. The first is that of the Sociology of Regulation and the opposing construct is based on the Sociology of Radical Change. The two views are based on the beliefs held by sociologists about the nature of society. One view is that of 'regulation' whereby society is seen in terms of unity and cohesiveness. In other words, the glue of society is analysed in an attempt to see what holds it together. They want to identify the pieces that hold society together. The other view is to identify the deep-seated structural constructs which give rise to conflicts and domination in society. This group is concerned with possibilities for change within society. They are interested in exploring what could be the case consequently the concept of domination plays an important part.

Burrell and Morgan use the above two opposing categories and the objective/ subjective dichotomy to build a matrix with the result that there are four fundamentally different paradigms, each of which can be used as a guide to analyse social phenomena (See Figure 1). 


\section{Figure 1 Sociological Paradigms and Organisational Analysis}

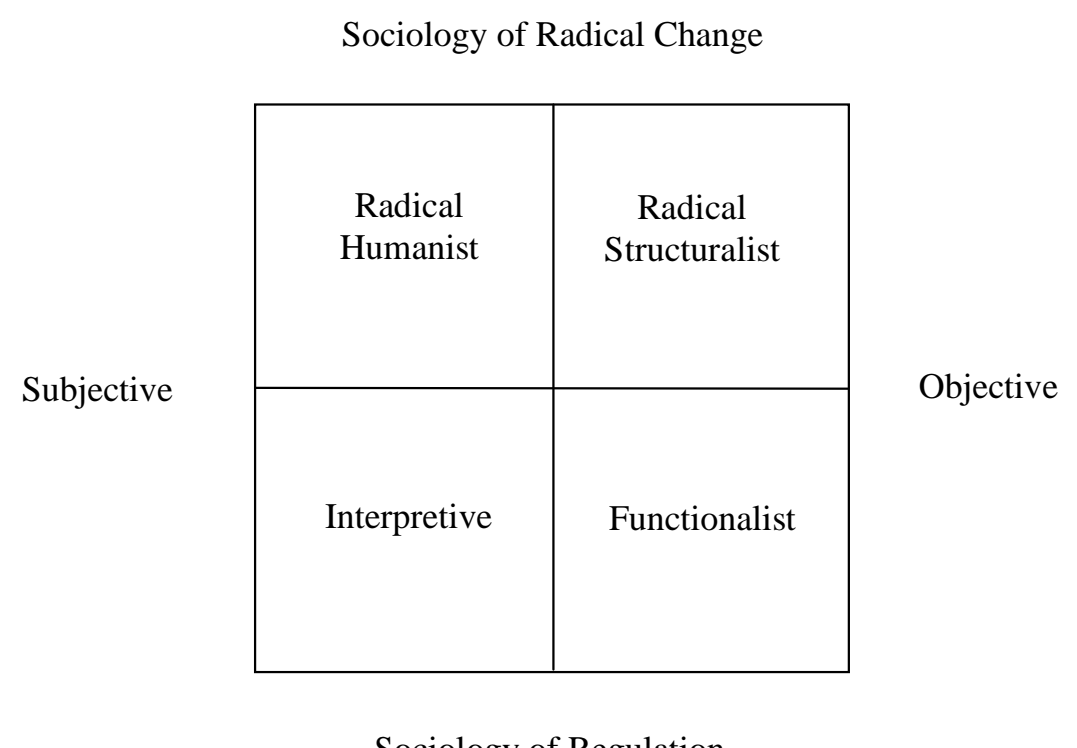

Sociology of Regulation

Source: Burrell and Morgan, 1979

The use of the word 'paradigm' has gained an increasing amount of usage in management and marketing circles but it is often used inappropriately. Kuhn, as has been discussed in this chapter, uses the word 'paradigm' to denote the way scientific practice is carried out at a given time to provide problems and solutions. In other words, one scientific approach is replaced over time by another new approach. Burrell and Morgan, on the other hand, use the term 'paradigm' in a broader sense to mean 'alternative realities', 'frames of reference', or 'universe of discourse'. In other words, Burrell and Morgan believe in multiple approaches to generate knowledge whereas Kuhn argues that one approach is replaced by another. 
Burrell and Morgan explore the basic meta-theoretical assumptions that comprise the underlying constructs of a paradigm. They attempt to diagnose the way that theorising is undertaken, and the manner in which research is conducted by social scientists working in each quadrant of the matrix.

A researcher in the Functionalist Paradigm seeks to find rational explanations to social/societal issues and problems. They assume that the world has relatively concrete structures and relationships which can be identified and measured using methods obtained from the natural sciences. On the whole the research is of a pragmatic nature whereby the analysis produces useful knowledge. Neo-classical economics would be an example of a school of thought operating in this quadrant.

In the Interpretive Paradigm researchers use subjective experience to understand the nature of the social world. They are participants in the research process rather than being just observers of the phenomenon under investigation. 'The interpretive researcher seeks to deconstruct the phenomenological processes through which shared realities are created, sustained and changed' (Hassard, 1991 p. 277). Phenomenology would be an example of a school of thought operating in this quadrant.

Researchers in the third quadrant, Radical Humanist, are like those in the interpretive paradigm in respect that they view the social world as being socially constructed. However, the construction is that of 'pathology of consciousness'. This approach highlights the alienating modes of thought in the modern capitalist world. In 
contrast, the Radical Humanist perspective explores the nature of change, emancipation, deprivation, and potentiality existing in society. They wish to identify the constraints placed on human development. An example of a school of thought operating in this quadrant is French Existentialism.

The final quadrant is that of Radical Structuralism. This group concentrates on structural relationships within a realist social world, comprising of 'facts' which have an existence of their own. However, this reality is made up of tensions and contradictions that lead, in a deterministic sense, to radical change. The construct of change is seen as a basic ingredient of the structure of society.

Whilst Burrell and Morgan and Kuhn are concerned with the production of knowledge, they each use the word 'paradigm' differently. Burrell and Morgan use the term paradigm in a broader sense than Kuhn. They use the term as a classificatory device to structure their thesis, in other words, to outline a map or framework of different types of social theory. 'Burrell and Morgan's 'paradigms' are ideal types of opposing metatheoretical assumptions and not, as they are for Kuhn, actual opposing substantive explanations' (Marsden, 1993 p. 99). Therefore, Burrell and Morgan's paradigms exist simultaneously and in perpetuity. Compared with Kuhn, they insist that the paradigms exist independently and maintain their own identity. Therefore, incommensurability, in some sense of the word, is a permanent feature of their model.

Each paradigm represents an ideology, with prefigured analysis which can be applied to the same phenomena, including marketing. Paradigms are social constructions 
reflecting the values and interests of a group of researchers who are working in a certain domain. Consequently, a paradigm is shared within a reference group or community of scholars.

There are numerous benefits to be gained from using the Burrell and Morgan matrix. According to Jackson and Carter (1991), the matrix saves having to justify an approach every time a new study commences or a report is published. By stating where the research sits in terms of the quadrant, the different ontological, epistemological, and methodological assumptions are implied.

Another role of the model, when undertaking a pluralist approach in a research programme, is to protect the weaker paradigm from the dominant paradigm which could easily take over and force the weaker paradigm to take backstage. This problem can be seen in the marketing context, where the dominant paradigm, based on performance and control in defence of a set of ideological values, makes it hard to gain a unified body of knowledge using alternative paradigms.

Jackson and Carter also claim that scientists can be seen as neutral agents thereby not having to take moral responsibility for the effects of their findings. This may be the case for positivists who have distanced themselves from metaphysical issues, such as ethics, thereby absolving themselves for the consequences of their findings. But, as we have seen with the Paradigms model, knowledge production is fragmented with no single homogeneous body of scientific knowledge. Therefore it is hard to argue that scientists are neutral agents. It has been argued in this chapter that no observation is theory independent. This means that a researcher brings to the 
problem their own pre-conceived ideas, so when making an observation certain phenomenon will be included by the researcher, whilst other phenomena will be excluded. As noted by Arndt, 'Paradigms are not value free and neutral. Rather, paradigms may be viewed as social constructions reflecting the values and interests of the dominant researchers in a science and their reference groups' (Arndt, $1985 \mathrm{p}$. $11)^{6}$

On the other hand there are some issues surrounding Paradigms that need to be discussed. First, do the divisions as outlined in Table 2 require exclusive forms of research programmes or is it possible that a research programme may involve one or more of the dichotomies? But then the question is in what combination? Another question is whether all dichotomies are necessary in a research programme?

In raising these issues, one's attention is drawn to the fact that the dimensions as outlined are based on a dualism, which by definition become mutually exclusive (Weaver and Gioia, 1994). However, it will be argued that in Chapter Five that such dualities are based on Cartesian dualism and as such can be repudiated. In other words, dualisms as outlined are not mutually exclusive and consequently it is possible to work within a combination of different dimensions.

Second, typologies in general are very descriptive, as stated by Jackson and Carter (1993), in replying to criticisms from Hugh Willmott, who believes the Paradigms model is prescriptive. In terms of the Paradigms model being descriptive, it will be argued later in the chapter, where the work of Deetz is discussed, that the Paradigms

\footnotetext{
${ }^{6}$ Note that the subjective nature of research will be explored in more detail in Chapter Three.
} 
model is inadequate as it fails to take into account all research positions, for example, postmodernism. On the other hand, the Paradigms model is not prescriptive in a formal sense but as discussed in this chapter, the model has a political genesis as it was written to warn researchers in organisation studies of the hegemony of positivism. By outlining other modes of research, Paradigms is drawing attention to other possible and equally valid ways of generating knowledge.

\section{Table 2 Common Divisions Affecting Organizational Inquiry}

$\begin{array}{lrrrr}\text { Structure } & \leftarrow & \text { versus } & \rightarrow & \text { Agency } \\ \text { Structural Functionalism } & \leftarrow & \text { versus } & \rightarrow & \text { Interpretivism } \\ \text { Determinism } & \leftarrow & \text { versus } & \rightarrow & \text { Voluntarism } \\ \text { Causation } & \leftarrow & \text { versus } & \rightarrow & \text { Meaning } \\ \text { Holism } & \leftarrow & \text { versus } & \rightarrow & \text { Individualism } \\ \text { Object } & \leftarrow & \text { versus } & \rightarrow & \text { Subject } \\ \text { Description } & \leftarrow & \text { versus } & \rightarrow & \text { Prescription }\end{array}$

Source: Weaver and Gioa 1994

Third, the objective/subjective dichotomy is designed to parallel the fact and value distinction developed by the positivists. Kavanagh (1994) believes the dichotomy reflects the realism-relativism debate. According to Rorty (1979), it would appear that the distinction creates more difficulties than it resolves. The objective/subjective dichotomy needs to be seen as a continuum, with strong and weak positions on that continuum. In this sense, it is not just an either/or phenomenon. Connell and Nord (1996) make the point that the objective/subjective continuum is both logically and 
practically unsound, as there are no ontological positions between the two poles, and many writers do not easily fit into one or the other 'camp'. The regulation-radical change dichotomy is also challenged by Connell and Nord (1996) as they see that researchers are not based on opposing assumptions but more on the interests of the researchers.

Fourth, Paradigms allows the development of paradigms or approaches to research without the influence of a dominant framework. However, it is debatable whether a two-by-two matrix is needed to make sense of the debate. Jackson and Carter (1991) argue that Paradigms allows researchers to adopt an approach without having to always justify the ontological, epistemological, and methodological foundations. However, Paradigms constrains theory development because its constructs are based on polarized conceptions of science and society, as noted above. As Willmott (1993, p. 682) points out, 'Paradigms assumes and strongly endorses a restriction of analysis within the confines of four, mutually exclusive 'ways of seeing'. A downside of accepting different paradigms is that compartmentalisation (Mouzelis, 2000) develops between the approaches, with each claiming a monopoly on truth. Unfortunately, this can lead to barriers being erected between the paradigms resulting in the negation of open-ended communication.

Willmott (1993) has also pointed out that if they had paid closer attention to Kuhn they would have realised that theory development is a process of struggles within existing theories that stimulate interest in alternative theorising. Kuhn recognised that there was discontinuity as well as incommensurability in this 'revolutionary' process. Therefore, we can cast doubt upon Paradigms' mutual exclusivity thesis. 
It would seem that the idea of Paradigms is appealing as a heuristic but this was not the intention of Burrell and Morgan. They believed that research must fit within the structure of the matrix. 'To be located in a particular paradigm is to view the world in a particular way. The four paradigms thus define four views of the social world based upon different meta-theoretical assumptions with regard to the nature of science and society' (Burrell and Morgan, 1979 p. 24). Burrell and Morgan conflate ontology and epistemology. As Kavanagh (1994) points out, the discussion of ontology only takes three paragraphs of a 300-page book. The framework itself is a paradigm in a strict Kuhnian sense. The four quadrants offered by Burrell and Morgan are just puzzle-solving methodologies, rather than a 'paradigm' shift, unlike sceptical post-modernism, which offers a completely new way of looking at issues, and is a paradigm shift in the sense outlined above.

Another interpretation of Paradigms is to see it in a historical context. That is to say that the matrix was developed to overcome the domination of functionalism in organisation studies. However, whilst this may be the case, the confines of Paradigms is a high price to pay to deal a blow to functionalism (Willmott, 1993).

The question can be asked whether Paradigms is a debate between different methodologies or a knowledge-building process with a philosophical orientation. It would seem that, given the above discussion, it is more the former than the latter. But despite all the criticisms, Paradigms has had a positive effect on marketing due to the work of Johan Arndt, and consequently it has allowed room for some limited discussion. 


\section{Arndt}

Johan Arndt (1985) has adopted the Burrell and Morgan (1979) framework and applied it to the marketing discipline, as detailed in Figure 2 below. Within each quadrant is the name of the paradigm and the type of methodologies that can be utilised by marketing researchers working within that particular paradigm. As will be noticed, the labels outside the matrix indicate the metaphors, based on the work of Morgan (1980) that can be used as a means to understand the dynamics of the different paradigms The four quadrants will now be described, followed by a brief description of the metaphors which relate to each quadrant.

For Arndt, the logical empiricism paradigm is basically a methodological approach where measurability is the key. Certain variables and relations are highlighted while excluding other variables that are deemed to be outside the construct being observed. Marketing phenomena are taken to have a concrete, real existence that is independent of the observer. The task of the researcher is to observe regularities in marketing behaviour or practice, with results which are value-free, objective and true.

Researchers in the socio-political quadrant are searching for predictable uniformities in marketing behaviour, again with concrete data that is measurable. However, the emphasis is on conflicts which arise between marketing actors often caused by the scarce allocation of resources.

The Subjective world quadrant is based on an interpretive paradigm. One such school is the social constructionists who believe that social reality is the product of 
subjective and intersubjective experience of the individuals involved in the marketing exchange. Hence, phenomena are understood from the viewpoint of the participant.

The Liberating quadrant describes conflicts and contradictions in the system. Researchers are primarily interested in ways that emancipation can be achieved for the actors in the exchange process. Therefore, there is a need not only to identify the actors but also the goals, interests and power bases of those involved in the marketing exchange.

Metaphors are a useful device to make sense of the world as they link the subjective and objective worlds with the use of symbolic constructs (Morgan, 1980). Language and images are used to view the world but it needs to be remembered that no single metaphor can capture an aspect in its entirety, so they are limited in certain respects. Therefore, using different metaphors to explain the dynamics of a paradigm, that is, using a pluralistic perspective, leads to a more meaningful understanding. 


\section{Figure 2 Paradigms and Metaphors in Marketing}

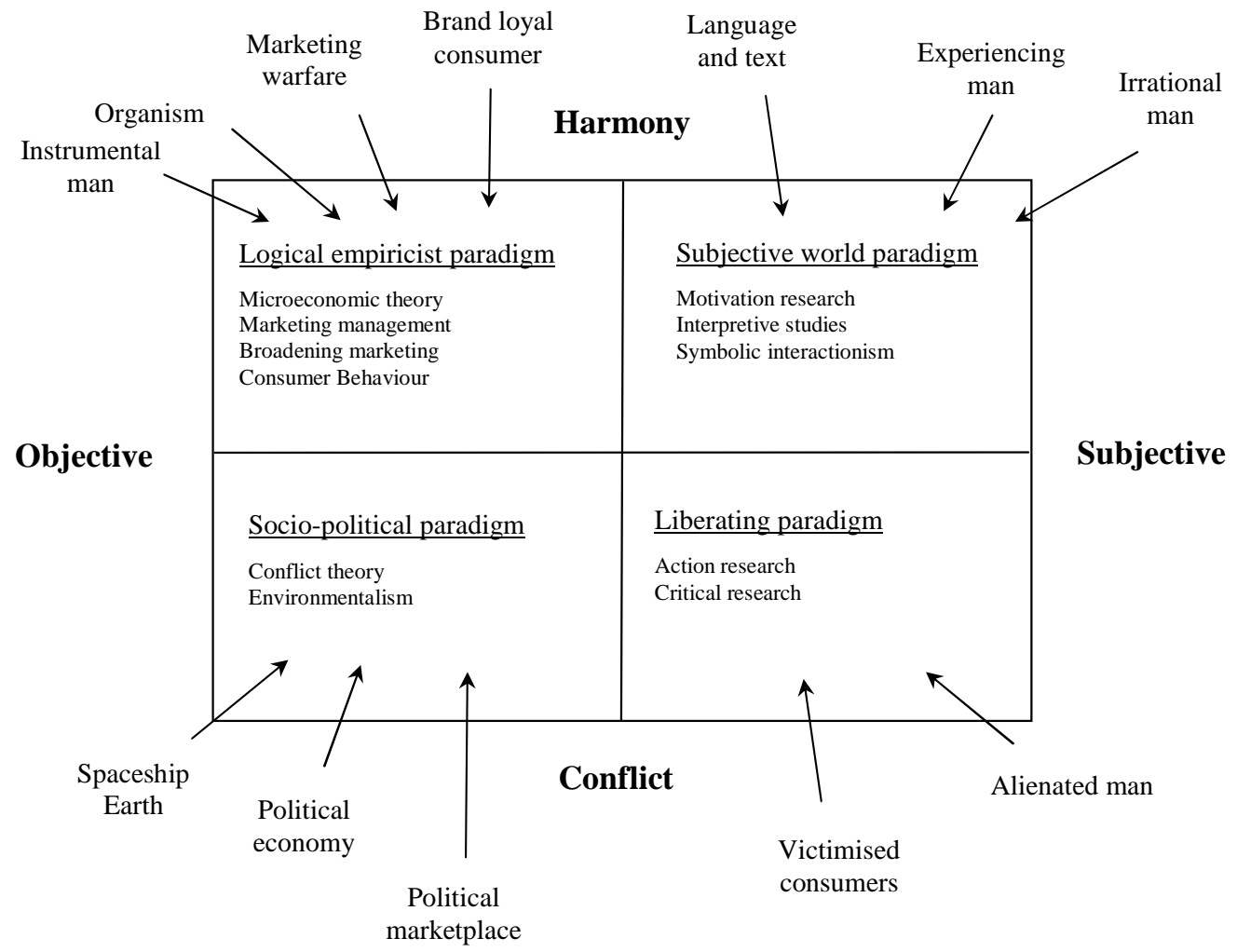

Source: Johan Arndt 1985

For Arndt, the logical empiricist paradigm is underpinned by micro-economics, with its emphasis on supply and demand curves, marginal analysis and utility maximisation, and cost functions. Therefore, one metaphor is that of the 'instrumental man' where the concern is with the development of purposive decision making. An example of a text book utilising this approach is Phillip Kotler's 
'Marketing Management', which uses the 4 p's framework to explore decision making in the marketing context. Another metaphor is that of 'marketing warfare' where military terms are used: for example, campaigns, tactics, strategy, and guerrilla activity. Authors such as Michael Porter (Competitive Strategy) and Ries and Trout (Positioning: the Battle for your Mind) are examples of work using such a metaphor. For example, in 'Competitive Strategy' the emphasis is on the firm gaining a sustainable competitive advantage at the expense of the competition.

The focus of the Socio-political paradigm is the interplay of power and those that wield power. One metaphor is that of the 'political marketplace' where the attempt is to capture the conflict that arises when actors are forced to scramble for scarce resources. An example of this type of research is that by Motiram and Vakulabharanam (2007), who discuss the marketing arrangements for small farmers in developing countries, where resources are scarce. Their research indicates that cooperation between farmer's leads to a more favourable outcome than the adoption of other models, such as a corporate structure.

Another perspective in this paradigm is the spaceship earth metaphor where the emphasis is on the need to obtain a balance in the ecological system. Woodside, Caldwell, and Spicer (2006) used ecological systems theory to explain the thoughts and actions of individuals with respect to decisions in lifestyle, leisure and travel choices. This research is interesting given the current debate in the media regarding carbon foot prints and air miles. 
The interest in the Subjective World paradigm is on the game of words, actions and thoughts of those involved in the marketing exchange. The 'experiencing man' metaphor is evoked to gain insights into the everyday experience of the consumer and also members of an organisation involved in marketing actions. This research approach uses phenomenology to explain consumer behaviour, and the use of semiotics to explain signs and symbols. An example of this type of approach is an article by Harvey and Evans (2001), who report on a project developed for Guinness PLC using semiotics, that is, the study of signs, symbols, words and pictures that create meaning and invoke feelings for consumers. The project developed a protocol for marketers in Guinness to gain an understanding of the reaction of consumers to competitor's advertisements, and then plot their propositions accordingly, thus gaining an insight into the competitive environment.

Another metaphor to explain the Subjective World paradigm is that of language and text, whereby storytelling is used to relate to the consumption of brands. An example of this approach is that by Papadatos (2006), who used stories elicited from customers' of Canada's Air Miles Reward Programme to gain insights into their lives so that the company could incorporate the information in their brand strategies.

The Liberating paradigm is concerned with the alienating role of marketing in society and questioning the value premises of marketing. Therefore the 'alienated man' metaphor is used which assumes man's role in the marketing process is passive, without the means to analyse and assess the process for themselves. An example of work exemplifying this metaphor is that of Davies and Elliott (2006). Using oral history as a research methodology, they interviewed a group of women of 
varying ages to understand the changing role of brands with regard to their shopping experiences over a number of decades. The key finding of the research was that whilst brand choice can be seen as empowering consumers, the responsibility of such decisions was not readily accepted by the consumers, as increased choice was seen as challenging and often confusing. The research found that empowerment is a paradoxical process and the results challenge the linear benefit assumption of classical economic theory.

Another metaphor in this quadrant is that of the 'victimized consumers' who are unable to cope due to their being disadvantaged but at the same time being bombarded with advertising images about 'the good life'. Critical theory is an example of this type of research. Such an approach undertakes a critique of the ideological basis of marketing (Burton, 2001). This involves a critical analysis of the underlying assumptions and beliefs regarding the marketing processes taking into account the historical and socio-economic environment. However, Burton (2001) makes the point that marketing academics have been criticized by academics outside the discipline for not adopting critical perspectives in their work. This is backed up by Saren, who states 'As the convenor of the marketing stream convenor of all five conferences to date, I feel qualified to observe that marketing has lagged some way behind other academic management disciplines in this area, both in volume and visibility' (Saren, 2006 p. 13). However, especially in the United Kingdom and the Continent there is a growing number of academics who are showing an interest in this area. ${ }^{7}$

\footnotetext{
${ }^{7}$ Two books in this area are to be published in 2008, namely 'Critical Marketing: Defining the field' by Michael Saren, Pauline Maclaran, Christina Goulding, Richard Elliott, Avi Shankar, Miriam Caterall; and 'Critical Marketing: Issues in Contemporary Marketing' edited by Mark Tadajewski and Douglas Brownlie.
} 
Unlike the discipline of Organisational Behaviour, the marketing literature unfortunately has not discussed in any depth Arndt's framework and the implications in adopting one research strategy over another. As will be argued in the next chapter, the dominant paradigm has been based on the logical empiricist paradigm. Arndt's article is disappointing in itself as there is no discussion of the different paradigms at a philosophical level. Apart from describing the methodologies that can be used, there is no discussion of the ontological and epistemological assumptions.

\section{Deetz}

One of the major problems with Paradigms is the objective/subjective distinction. As will be explained in greater detail in Chapters Four and Five, the distinction is based on a false dualism.

Notwithstanding the above claim, according to Deetz (1996) the objective/subjective dichotomy has other issues that need to be addressed. First, the objective/subjective labels are not a natural fact but are socially constructed. 'In so-called "objective" research, concepts and methods are held a priori, are unknown projections of researchers' own ways of encountering the world, countering the world as observed without ownership or critical reflection, and are not subject to the "objection" of the outside toward possible alternatively constituted worlds' (Deetz, 1996 p. 193). The interpretivists have better claim to being objective as they bring into account other language games and other ways to approach research thus denying a privileged position. 
Second, the nature of the object/subject split leads to a type of neo-positivism being practised. Such a position is protected by the framework and the same would be said for the interpretivists.

Another criticism is that new approaches, based on the linguistic turn in modern philosophy, such as feminists, critical theorists and postmodernists, are not able to be placed on the objective/subjective continuum.

An additional issue is that the framework has led to an artificial distinction and conflict between quantitative and qualitative types of research. Neo-positivists think that the two types of research represent different ways to collect data and they utilise the concept of triangulation to gain different insights to the same phenomenon. However, such an approach denies the basic conceptual differences that are inherent in each approach. 'The modes of analysis do not work from different points of view on the same thing; they are producing and elaborating in the act of researching different phenomena for different reasons' (Deetz, 1996 p. 194).

In contrast to the Paradigms framework, Deetz (1996) developed a framework based on the linguistic turn in philosophy. The aim was to draw attention to differences and similarities rather than to develop a rigid typology. 
Figure 3 Characteristics of the Local/Emergent-Elite/A Priori Dimension

\section{Local/Emergent}

Elite/A Priori

\begin{tabular}{|l|l|}
\hline Comparative communities & Privileged community \\
\hline Multiple language games & Fixed Language Game \\
\hline Particularistic & Universalistic \\
\hline $\begin{array}{l}\text { Systematic philosophy as } \\
\text { ethnocentric }\end{array}$ & $\begin{array}{l}\text { Grounded in hoped for } \\
\text { systematic philosophy }\end{array}$ \\
\hline Atheoretical & Theory Driven \\
\hline $\begin{array}{l}\text { Situationally or structural } \\
\text { determinism }\end{array}$ & Methodological determinism \\
\hline Nonfoundational & Foundational \\
\hline Local narratives & $\begin{array}{l}\text { Grand narrative of progress and } \\
\text { emancipation }\end{array}$ \\
\hline $\begin{array}{l}\text { Sensuality and meaning as } \\
\text { central concerns }\end{array}$ & $\begin{array}{l}\text { Rationality and truth as central } \\
\text { concerns }\end{array}$ \\
\hline Situated, practical knowledge & $\begin{array}{l}\text { Generalizable, theoretical } \\
\text { knowledge }\end{array}$ \\
\hline $\begin{array}{l}\text { Tends to be feminine in } \\
\text { attitude }\end{array}$ & $\begin{array}{l}\text { Tends to be masculine in } \\
\text { attitude }\end{array}$ \\
\hline Sees the strange & Sees the familiar \\
\hline Proceeds from the other & Proceeds from the self \\
\hline $\begin{array}{l}\text { Ontology of "otherness" over } \\
\text { method }\end{array}$ & $\begin{array}{l}\text { Epistemological and procedural } \\
\text { issues rule over substantive } \\
\text { assumptions }\end{array}$ \\
\hline
\end{tabular}

Source: Deetz, 1996 p. 195

The elite/a priori dimension is based on a privileged language system. Language is

held as a constant and the research programme is theory driven with definitions identified before the process begins. Also there is a tendency to generalise the research findings based on essentialist assumptions. On the other hand, the local/emergent dimension is based on an open language system where research is 
conducted as a first slice of a problem or issue so nothing is definitive and new meanings are generated as the research programme progresses. The research findings are particularistic and the objective is to gain insights, which may or may not be carried over into similar situations. 'The research attends to the feelings, intuitions and multiple forms of rationality of both the researched and researcher rather than using a single logic of objectification or purified rationality' (Deetz, 1996 p. 196).

The continuum has three advantages. First it acknowledges the linguistic dimension of all research approaches. Second, it establishes different kinds of knowledge generation, that is, from codified knowledge to practical knowledge. Finally, the continuum shows the political nature implicit in all research. In other words, knowledge generation is biased towards a community including managerial issues and problems.

The second dimension is similar to the change/regulation of the Paradigms framework but is about the way discourse is viewed, that is, as being unitary or displaying difference (see Figure 4). With the consensus dimension, order is the dominant feature whereas with the dissensus dimension the discourse is about struggle and conflict. Therefore the task of the researcher is to unearth tension that exists within an organisation. 'The mirror gives way to the "lens" as the metaphor noting the shifting analytic attempt to see what could not be seen before and showing the researcher as positioned and active' (Deetz, 1996 p. 197). 


\section{Figure 4 Characteristics of the Consensus-Dissensus Dimension}

Consensus

\begin{tabular}{|l|l|}
\hline Trust & Suspicion \\
\hline $\begin{array}{l}\text { Hegemonic order as natural } \\
\text { state }\end{array}$ & $\begin{array}{l}\text { Conflicts over order as natural } \\
\text { state }\end{array}$ \\
\hline Naturalization of present & $\begin{array}{l}\text { Present order is historicized and } \\
\text { politicized }\end{array}$ \\
\hline $\begin{array}{l}\text { Integration and harmony are } \\
\text { possible }\end{array}$ & $\begin{array}{l}\text { Order indicates domination and } \\
\text { suppressed conflicts }\end{array}$ \\
\hline $\begin{array}{l}\text { Research focuses on } \\
\text { representation }\end{array}$ & $\begin{array}{l}\text { Research focused on challenge } \\
\text { and reconsideration } \\
\text { (representation) }\end{array}$ \\
\hline $\begin{array}{l}\text { Mirror (reflecting) dominant } \\
\text { metaphor }\end{array}$ & $\begin{array}{l}\text { Lens (seeing/reading as) } \\
\text { dominant metaphor }\end{array}$ \\
\hline $\begin{array}{l}\text { Validity central concern } \\
\text { Researcher anonymous and out } \\
\text { of time and space }\end{array}$ & $\begin{array}{l}\text { Insight and praxis central } \\
\text { concern }\end{array}$ \\
\hline pheositioned as abstraction & Theory as opening \\
\hline $\begin{array}{l}\text { Unified science and } \\
\text { triangulation }\end{array}$ & Positional complementarity \\
\hline Science as neutral & Science is political \\
\hline Life is discovery & Life is struggle and creation \\
\hline Autonomous/free agent & Historically/socially situated \\
\hline agent
\end{tabular}

Source: Deetz, 1996 p. 197 
From the above two dimensions four distinct discourses have been developed, as illustrated in Figure 5.

Figure 5 Contrasting Dimensions from the Metatheory of Representational Practices

\section{Relation to Dominant \\ Social Discourse}

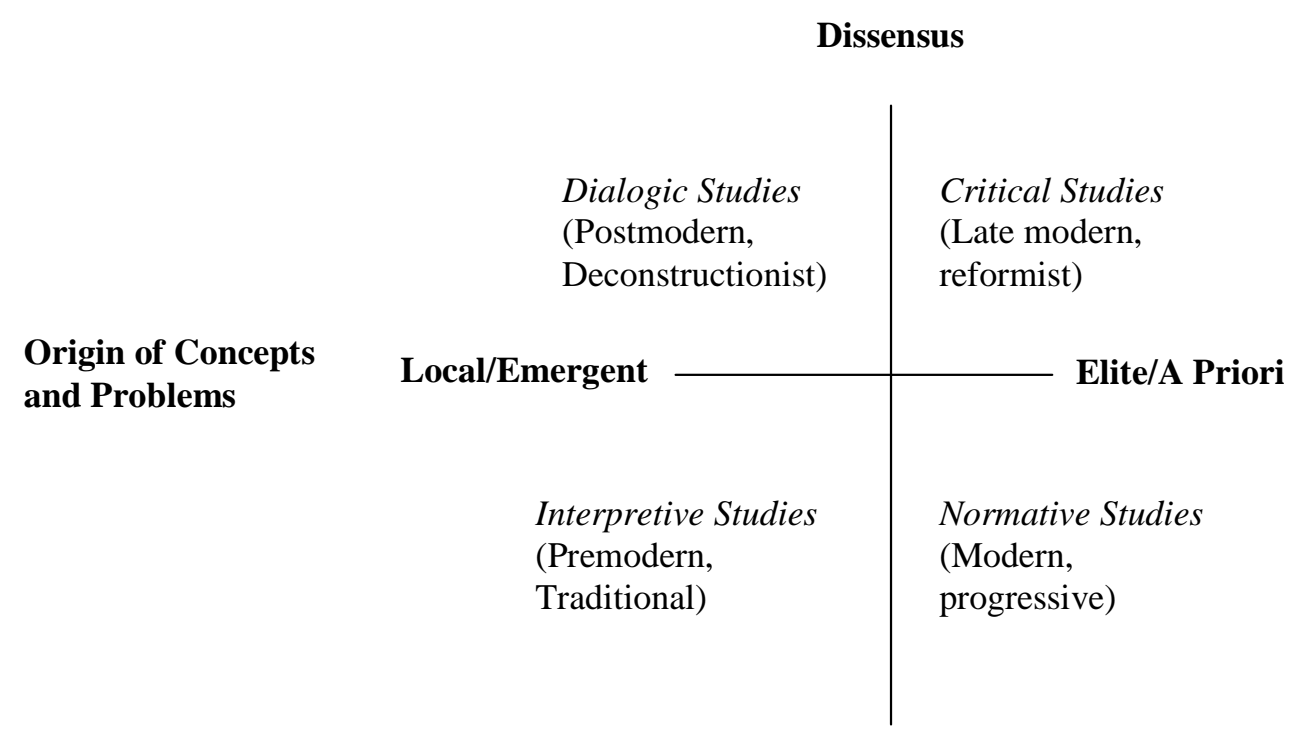

Consensus

Source: Deetz, 1996 p. 198

According to Deetz (1996), it would be unwise to think that the four quadrants represent distinct paradigms for the following reasons. First, the four discourses are not discreet as conflict and strife reside within them. Second, there is no clear demarcation between the four discourses as there is an overlap between them. 'Most 
researchers and teachers do not cluster around a prototype of each but gather at the crossroads, mix metaphors, borrow lines from other discourses, and dodging criticism by co-optation' (Deetz, 1996 p. 199). Finally, working within a discourse does not mean that you are sealed off from work in other areas. Borrowing of insights occurs and struggles between the discourses take place.

It is argued that the Deetz framework, as outlined above, is adopted as the basis to explore a new approach that will generate marketing management discourse based on praxis because it is based on the linguistic turn in modern philosophy.

However, the frameworks as outlined above raise some further issues that need to be discussed. Can researchers work in one or more paradigms at a time? Or can a researcher understand the work being undertaken in a different paradigm than their own modus operandi? In other words, the issue of incommensurability needs to be addressed.

\section{Incommensurability}

The purpose of this section is to explore the problem of incommensurability, which states that there are no objective standards available to determine if one theory is better than another. It is believed that paradigms differ because they embody different ways of describing the world, consequently standards used within a paradigm cannot be justified outside that paradigm. Also, theories created within a specific paradigm as well as the values, interests and cultures that form part of that particular paradigm cannot be judged from a standpoint outside that paradigm. 
Given that the Deetz (1996) framework is to be used to explore a new paradigm for the marketing discipline, the question arises whether it is possible to make sense of competing paradigms? Furthermore, is meaningful debate between paradigms possible? To answer these two questions it is necessary to explore in more detail the problem of incommensurability.

This problem has been at the forefront of management debate over the last twenty years (Jackson \& Carter, 1991; Cannella \& Paetzold, 1994; Hassard, 1991; Pfeffer, 1993; Poropat, 2002; Scherer \& Steinmann, 1999; Schultz \& Hatch, 1996, Weaver \& Gioia, 1994; Willmott, 1993) leading to what has generally been called paradigm wars. However, the problem of incommensurability within the marketing discipline has not lead to a similar debate. Instead, the argument has been between positivism and interpretivism (Davies \& Fitchett, 2005), and carried out by the two main protagonists, Hunt (1983) and Anderson (1983). This important debate within the marketing literature will be discussed in further detail in the Chapter Three. Nonetheless, it needs to be noted that the incommensurability debate has been generated mostly by a desire to select the 'right' paradigm, that is, the one that will lead to some notion of 'truth' ${ }^{8}$ However, other concerns have also arisen.

Pfeffer (1993) argues that incommensurability should be ignored and that the management discipline should pursue an isolationist approach. He believes that a minimal level of consensus is needed to develop knowledge within a given field as the developmental process is on-going and cumulative. By developing a consensus in a field of study, legitimacy is developed as an elite is established who in turn

\footnotetext{
${ }^{8}$ This is an important issue and will be addressed in Chapter Five and Chapter Eight, where a pluralistic approach to marketing will be argued for, and if adopted, the problem of incommensurability will be less of an issue.
} 
become gatekeepers for the discipline, setting and enforcing methodological standards. On the other hand, as Jackson and Carter (1991) argue, if one rejects paradigm incommensurability it is one small step to accepting an authoritative science, which means long term the discipline would stagnate due to the lack of new ideas and concepts.

Nonetheless, there are a number of different meanings of the term 'incommensurability'. Experiential incommensurability (Poropat, 2002) is where one person's experience cannot be directly compared with another person's experience. For example, the taste I obtain from eating an apple will be different from the taste you experience when eating an apple, even if it is the same apple. There is no way we can say, without doubt, that we had the same taste experience. On the whole, individual biases are involved so there are no 'objective' criteria available to make an assessment. This is often the case when wine judges give different scores to the same wine.

However, Kuhn saw the issue of incommensurability as a specific linguistic thesis and so any contradiction is a relation between statements. Incommensurability therefore avoids a contradiction between paradigms. He argues that paradigms are incommensurable, that is, observations and understandings in one paradigm cannot be meaningfully translated into another paradigm because their basic assumptions cannot be reconciled. The case of relativity versus classical mechanics is an example of two incommensurable frameworks. Other examples are the quantum theory versus classical mechanics and the impetus theory versus Newton's mechanics. 'In learning a paradigm the scientist acquires theory, methods and standards together, usually in an inextricable mixture...That observation ... provides 
our first explicit indication of why the choice between competing paradigms regularly raises questions that cannot be resolved by the criteria of normal science ... Like the issue of competing standards, [it] can be answered only in terms of criteria that lie outside of normal science altogether, and it is that recourse to external criteria that most obviously makes paradigm debates revolutionary' (Kuhn, 1962 pp. 109-110).

For Kuhn, commensurability is achieved when the revolutionary science wins the struggle and consequently becomes the 'normal' or dominant science. However, Burrell and Morgan warn against Kuhn's type of commensurability as they are against the domination of a particular paradigm, in their case, particularly functionalism. The difference is that Kuhn sees paradigms as being incommensurable and consequently the new paradigm replaces the old one, whereas Burrell and Morgan see a number of paradigms existing separately over a long period of time.

One of the problems regarding the term 'incommensurability', according to Bernstein (1983), is that there is not a well-defined statement acceptable to both relativists and objectivists which encompasses what the term means. For Bernstein, a distinction needs to be made between incompatibility, incommensurability, and incomparability. Paradigms are incompatible when a logical contradiction exists. If the history of science is viewed from a linear point of view there is the assumption that there is a common logical framework. However, such a view omits the element of conflict and destruction of different theories and paradigms.

'We must avoid the fallacy of thinking that since there is no fixed, determinate rules for distinguishing better from worse interpretations, there is consequently no rational way of making or warranting such practical comparative judgements' (Bernstein, 1983 p. 91). 
In other words, paradigms can be compared and contrasted on rational grounds as long as the premises are made clear, and these premises are based on a practical basis. The notion of a practical basis will be explored in more detail when the concept of praxis is discussed in Chapter Five.

Turning to the problem of incommensurability, Bernstein (1983) makes it clear that there is no permanent neutral observation language that can be used to evaluate rival positions. Kuhn was challenging the dogma of empiricism, which believes that there is something permanent and determinate that remains intact when making comparisons. Kuhn argues that there is no algorithm for theory choice among competing paradigms.

'Kuhn did not introduce the incommensurability thesis in order to call into question the possibility of comparing theories and rationally evaluating them, but to clarify what we are doing when we compare theories ...... for Kuhn rival paradigm theories are logically incompatible (and, therefore, really in conflict with each other); incommensurable (and, therefore, they cannot always be measured against each point-by-point); and comparable (capable of being compared with each other in multiple ways without requiring the assumption that there is or must always be a common, fixed grid by which we measure progress)' (Bernstein, 1983 p. 86).

According to Scherer \& Steinmann (1999) three conditions must be present for paradigms to be considered incommensurable. First there must be a radical difference between the systems of orientation. Second, a conflict must exist between the systems, whereby a choice needs to be made regarding the action to be undertaken to do the research. Finally, pluralism of perspectives is another necessary condition. 
It needs to be noted that paradigms do overlap with respect to observations, concepts and problems. As Willmott (1993 p. 688) has noted, 'new paradigms do not parachute from the skies.' Theories that are thought to be incommensurable must share part of a common world otherwise there cannot be any sense to the idea of scientific change. For theories to clash they must be referring to a similar domain otherwise it would not make sense to say that they clashed. Clashed about what? '... all paradigms are mediated by others ....While Einsteinian physics broke profoundly with Newtonian physics, it none the less had direct continuities with it at the same time: if Protestantism differs in basic ways from Catholicism, the content of the former cannot be fully understood apart from its relation to the latter as critique' (Giddens, 1979 p. 144 emphasis in original).

Within the management and marketing disciplines there have been attempts to overcome the problem presented by incommensurability. This is despite the fact that incommensurability is a defining characteristic of the term 'paradigm'. Davies and Fitchett (2005) in an attempt to overcome what was seen as the constraints of incommensurability, contrasted two studies of the same phenomenon, one using a positivist approach and the other an interpretivist approach. They argue for a more fluid understanding of paradigms and believe that it is possible to bridge the gap between different aspects of research practices. It would seem that the approach is similar to a strategy identified by Schultz and Hatch (1996), termed 'parallel', as different paradigms are used simultaneously, and are treated as being on equal footing. However, whilst it is possible to compare and contrast two research programmes, what is not make clear is how a choice would be made if two studies claimed competing theories.

Another research strategy is the adoption of an integration approach (Willmott, 1993a), which attempts to assess and synthesize a variety of different approaches. 
However, as noted by Burrell and Morgan 'A synthesis is not possible, since in their pure form they [paradigms] are contradictory, being based on at least one set of opposing meta-theoretical assumptions. They are alternatives, in the sense that one can operate in different paradigms sequentially over time, but mutually exclusive, in the sense that one cannot operate in more than one paradigm at any given point in time, since accepting the assumptions of one, we defy the assumptions of all the others' (Burrell and Morgan, 1979 p. 25).

Schultz and Hatch (1996) have identified a sequential strategy, whereby specific paradigms are used complementarily rather than exclusively. The research may start with one paradigm, for example an interpretivist approach by gaining qualitative data, and then move on to a positive/functionalist approach where quantitative data is obtained to explain a causal relationship. Such an approach is linear and unidirectional. However, it needs to be noted that in many cases, the same paradigm is being used but only the methodology is different. In other words, the ontological and epistemological assumptions are the same, so the qualitative approach is justified in positivist terms, in terms of criteria such as validity, reliability and sampling methods employed.

Hassard (1991) proposes a new methodology using multiple paradigm approach based on the four paradigms of Burrell and Morgan. His aim is to attempt to overcome the relativism of Paradigms and 'the lack of a convincing metaparadigmatic alternative' (Parker and McHugh, 1991 p. 451). There a number of problems with the multi-paradigm approach. First, on what basis is the point of comparison determined to assess the mixture of the paradigms being considered? 
Additional reasoning regarding the criteria cannot be based on a multi-paradigm view as this would lead to a circular argument. Second, when two paradigms are in serious conflict and co-existence is not possible due to completely different value positions, the multi-paradigmatic approach is not a possibility.

Another problem is whether it is possible to read the relevant literature of a particular paradigm and then utilise the approach and apply it to a given research problem? If this approach was used to cover a number of different paradigms, as per Hassard, the researcher would need to change their core assumptions. Is this possible given it would entail a change of the researcher's beliefs about the external world, including their political orientation? To achieve this would require the researcher to cleanse themselves of any ethnocentric biases from their 'pre-entry' paradigmatic thinking to avoid any possible contamination.

Hassard's approach, however, may be just the application of different methodological approaches. Could it be that he utilises both a methodological and epistemological approach but not does include any ontological distinctions? If this is the case then such a multi-paradigmatic research agenda can be undertaken within a broad realist ontology, along with a pluralist political philosophy. However, a more important question is why would one want to undertake a study of the same phenomenon using different paradigms? Such an approach may give one insights but if one was to adopt a pluralistic approach then such an understanding can be gained by reading other peoples' research papers. 'It is only through transgressing boundaries that new and exciting forms of knowledge can emerge...' (Parker and McHugh, 1991 p. 455). 
To sum up this section, incommensurability poses a problem if and only if one wants to identify the 'right' approach to obtain 'truth'. Kuhn (1962) has attacked the enlightenment ideals by indicating the discourse for science is no different from the discourse for practical and aesthetic knowledge. Bernstein (1983) has shown that it is possible to compare and evaluate different paradigms, but there is no neutral observation language. In other words, paradigms can be compared and contrasted as long as the premises, based on a practical objective, are made explicit. The next section will discuss the psychological underpinnings of paradigm selection.

\section{Scientific Style}

Within the marketing literature there have been a number of studies that explore how marketing academics undertake their research. One study is by Franc and Mazanec (2005) where they report on an attempt to identify the scientific identity of marketing. This was achieved by conducting a quantitative study of research approaches of marketing scholars, based on the idea that they differ in their beliefs about the role of science in marketing. The study covered four dimensions of marketing, namely purpose (theoretical or pragmatic); Types of problems (broad or focused); ways of solving (quantitative or qualitative); philosophical underpinnings (objective or subjective).

Whilst the authors state that belief systems about science are heterogeneous, they do assume that marketing is a science. Also, they posit philosophical underpinnings being based on the dichotomy between objective and subjective, which is similar to the Paradigms typology. However, after reading the article and noting the 
descriptions of the six identities, which could be argued are not necessarily mutually exclusive, one is left with asking "So what?" It would seem that the article does not help in determining a more appropriate way to generate marketing knowledge.

However, another approach suggests that the type of paradigm that a researcher selects may be influenced by scientific style. Hirschman (1985) outlines four types of scientific style based on the personality types developed by Jung. The two constructs taken from Jung are about our preferences for handling information and our preferences for decision making. Each construct is a continuum with two end points. Therefore when testing for a personality type a candidate will lie somewhere on the continuum. In other words, it is not an extreme, either or situation, but as Jung notes it is not possible to work in both modes at the same time.

Individuals can obtain data either from the senses (Sensing) or by their imagination (Intuiting). The sensing individual will be more interested in details and the specifics of a situation and prefer to work with factual material. The intuiting individual will be more interested in the big picture and tend to concentrate on "possibilities" or hypothetical situations than the current facts.

Individuals tend to have a preference for the way data is handled regarding decisions. The two constructs used in decision making, which are antithetical to one another, are Thinking and Feeling. Thinking is analytical, formal and abstract ways of reasoning, seeking to explain events in logical, technical terms that are independent of human concerns. Feeling on the other hand bases decision making on personal value judgements, with an emphasis on ethical issues pertaining to an individual. 
The following two-by-two matrix is developed from the above descriptions:

Figure $6 \quad$ Four Scientific Styles

Data Acquisition

\begin{tabular}{|c|c|c|}
\hline & Sensing & Intuiting \\
\hline Thinking & $\begin{array}{l}\text { Analytical } \\
\text { Scientist }\end{array}$ & $\begin{array}{c}\text { Conceptual } \\
\text { Theorist }\end{array}$ \\
\hline \multicolumn{3}{|l|}{$\begin{array}{c}\text { Data } \\
\text { Process }\end{array}$} \\
\hline Process & & \\
\hline Feeling & $\begin{array}{l}\text { Particular } \\
\text { Humanist }\end{array}$ & $\begin{array}{c}\text { Conceptual } \\
\text { Humanist }\end{array}$ \\
\hline
\end{tabular}

Source: Adapted from Hirschman, 1985 p. 227

Given that the two constructs as outlined above are positioned at right angles to each other it is possible to obtain four quadrants with distinctive personality traits which can be equated with scientific style. This style is about the way a researcher prefers to undertake research. Descriptions of the four types as outlined in the matrix above are as follows. There are some issues that need to be addressed about the framework before a description of the four quadrants is provided. The framework is not to be seen as a straight-jacket where an individual researcher must fit, ant there will be overlaps with researchers working in both categories. However, the framework does allow for the understanding of knowledge production. Each approach, as outlined 
below, has characteristics which can make unique contributions to research so it cannot be claimed that one way is better than another. Each style provides a valuable way of interpreting reality.

Researchers in the Analytical Science quadrant see science as value-free and independent of an observer's perception. 'The strengths of the analytical scientist are an overarching push toward the rational explanation of phenomena, rigorous adherence to logic in the preparation of hypotheses, and a quest for accuracy, precision, and control in the conduct of research' (Hirschman, 1985 p. 228).

The Conceptual Theorist has a preference for speculative theorising and the development of novel concepts. The researcher in this quadrant views science as a means to identify problems and push the boundaries by generating new ideas that contradict the accepted viewpoint.

The Conceptual Humanist has preferences for utilising the thinking and feeling constructs. They view science as a value-laden endeavour based on personal social and political values. They are usually advocates of a particular theory or scientific position so become personally committed to the cause. If the topic is of no personal interest to them they are inclined to switch off. Their overarching drive is for human development and aesthetic beauty. 'Conceptual, rather than empirical, inquiry modes are favored by the conceptual humanist, and the preferred form of logic is the dialectic' (Hirschman, 1985 p. 232). 
Based on the constructs of sensing and feeling, the researcher in the Particular Humanist quadrant is concerned with conducting research on the basis of personal involvement. They attempt to seek insights into individuals by the use of qualitative methodologies such as case studies, in-depth interviews or participant observation. 'The intent of the researcher using these techniques is to empathetically comprehend the life of another individual or group. To the particular humanist, knowledge derives from personal contact and must be interpreted within the subjective context of both the observer and the observed' (Hirschman, 1985 p. 235).

The above is an interesting means to view the way that research can be conducted. By understanding the underlying constructs of information gathering (sensing and intuiting) and decision making (thinking and feeling) we can obtain insights as to why a research process is adopted. As Hirschman has noted, 'To advocate that consumer researchers become fluent in these additional modes of inquiry does not imply that all consumer researchers should necessarily practice these types of research, but it does imply they should be able to understand the processes underlying these alternative modes of inquiry and be able to comprehend and evaluate competently the results obtained by others' (Hirschman, 1985 p. 238).

We can also see that the framework is similar to Paradigms in the following way:

$\begin{array}{llll}\text { Analytical Scientist } & \leftarrow & \rightarrow & \text { Functionalist } \\ \text { Conceptual Theorist } & \leftarrow & \rightarrow & \text { Radical Humanist } \\ \text { Particular Humanist } & \leftarrow & \rightarrow & \text { Interpretivist } \\ \text { Conceptual Humanist } & \leftarrow & \rightarrow & \text { Radical Structuralist }\end{array}$


Research by Leong, Sheth \& Tan (1994) utilised the work of Hirschmann and conducted a study of academic members of the American Marketing Association. With a response of 269 academics the study explored the relationship between academic style and the type of research preferred by the respondent. 'The main findings of this study indicate that marketing academics most strongly endorse the research values and attitudes of the analytical scientists, but that they appeared to have personality types inconsistent with this style. Indeed, the distribution of personality types among the respondents surveyed suggested that the sensingthinking personality type of analytical scientist was least in evidence' (Leong et al, 1994 p. 21).

Leong's research also indicated that a larger number of senior academics were sensing-thinking types (analytical scientists) than their junior colleagues. It would seem by adopting a research programme academics become institutionlised and over time this affects their preferred research style. Leong also makes the point that doctoral programmes perhaps shape a researcher's style, as the methodology courses and the programmes in general endorse the values and attitudes of the analytical scientist. This may lead to academics being alienated from their preferred personality type by 'instructing them to conduct research in ways inconsistent with their own personal styles and values' (Leong et al, 1994 p. 22).

This section indicates that despite there being a number of different research approaches available, as outlined in the three frameworks outlined in this chapter, the paradigm that is undertaken by an academic will be conditioned by the dominant research programme in the discipline. The $\mathrm{PhD}$ programme, need for tenure, and 
progression through the system determines the research paradigm that is adopted, despite the personality traits of individual academics. It is now apposite that marketing's dominant research programme is examined.

\section{Conclusion}

The thesis argues that marketing management should adopt the linguistic turn in modern philosophy, but to justify this claim the philosophical underpinnings of marketing management need to be explored. With this in mind the work of Thomas Kuhn was explored to understand the dynamics of change within a discipline and also the paradigm concept. The Burrell and Morgan Paradigms framework was introduced, as well as the framework developed by Arndt, as a basis to explore different paradigms. The overriding benefit of the frameworks is that they give a brief overview of the different approaches than can be adopted when undertaking a research programme. However, Deetz shows that there are some serious shortcomings regarding the Burrell and Morgan framework, and by default the Arndt framework, so consequently developed an adapted framework based on the linguistic turn in modern philosophy. It is proposed that the Deetz framework will be used to explore the different paradigms currently being used in the marketing discipline as it is based on the linguistic turn in modern philosophy

The notion of incommensurability was then explained and different research strategies to overcome the problem of incommensurability were postulated. The conclusion was reached that incommensurability only becomes a problem if a foundational approach to knowledge is adopted. This does not involve an 'anything goes' approach but an exhortation for reflective discourse as a means to learn about 
different approaches and determine what may be the best approach to adopt so as to overcome the problems faced by the marketing discipline.

The next chapter is entitled the Dominant Paradigm and explores Scientific Realism and problems associated with empiricism in general. Within the chapter the problems of objectivity will be explored and Imre Lakatos' (1970) 'Methodology of Scientific Programmes' will be utilised to determine how well the dominant paradigm has served the marketing discipline. 


\section{Chapter 3: The Dominant Paradigm}

\section{Introduction}

The aim of this chapter is to review the dominant paradigm in marketing and also the main alternatives that have been published in the literature. Hunt (1994) believes that there is no dominant paradigm in marketing. However, this is contrary to a number of other authors (Arndt, 1985; Razzaque, 1998; Saren, 2000). It would seem that Hunt is restricting his definition of positivism, in respect that he writes mainly about logical positivism, rather than empiricism in general. It is worth remembering that information is not knowledge. Unfortunately a lot of marketing literature is information-increasing but not knowledge-extending (Skipper \& Hyman, 1990). According to Skipper and Hyman (1990), information is a measure of probability and is based on data at a given time. Information contains a degree of surprise and is not based necessarily on truth. For example, a statement containing the information 'The Russians are coming' has an element of surprise and is based on a probability at the time but it is not necessarily true. In marketing information is gathered but it takes a further step to turn information into knowledge. The use of deductive reasoning is a vital step in the process. ${ }^{9}$ By confining marketing research to the positivist framework the production of knowledge is confined to a limited subset of marketing problems. ' ...there have been disparate substantive contributions which have attempted to explain some small part of overall market reality. Also, attempts to develop an overarching, concatenated framework have been few and far between' (Deshpande, 1983 p. 106).

\footnotetext{
${ }^{9}$ For a further explanation of the necessity of using deductive reasoning in marketing refer to Skipper and Hyman (1987) 'Evaluating and Improving Argument-Centered Works in Marketing'.
} 
The method of traditional science is seen as the dispassionate, unbiased testing of hypotheses whereby replication is a paramount criterion for success. Such an approach is criticised as it ignores the social dimensions of knowing. It is an inaccurate description of not only the social world but also the way knowledge is acquired (Zaltman et al, 1982). According to Arndt (1983) 'no general marketing theories come close to satisfying a rigorous theory of science criteria.' However, subfields such as consumer behaviour have fared better but mainly by the process of borrowing theories from other disciplines (Razzaque, 1998; Murray et al, 1995).

The chapter begins by a discussion on empiricism in general, as well as a brief outline of logical positivism and logical empiricism, which sets the stage for an exploration of scientism as the foundation for marketing. After outlining the problems of adopting such an approach, the two major alternatives that are discussed in the literature are outlined, namely scientific realism and relativism. It is argued that the debate between the two research approaches has been reduced to the methodological level and has not furthered the development of theory in the discipline. The chapter concludes by an evaluation of the marketing management research programme using a framework proposed by Lakatos (1970).

\section{Marketing Science}

One of the perennial questions in the marketing discipline is whether it should be seen as an Art or a Science. This begs the question as to what counts as a 'science' or an 'art'? Unfortunately marketing literature has concentrated on the former issue, without any in-depth discussion about how marketing could be perceived as an art (Brown, 1996). Why has marketing upheld the sciences as an ideal when conducting 
research? Marketing has adopted this paradigm due to the applied nature of the discipline and also the perceived needs of academics to become respectable, as well as the needs of corporate executives or marketing practitioners who want "facts that work' (Willmott, 1999). Empiricism is cast as the ideal approach for both the natural and social sciences as its objective stance based on detached observation ensures ‘intersubjective certification' (Johan Arndt, 1985). By adopting the principles of traditional science, namely that it is dispassionate, value-neutral, with the unbiased formulation and testing of hypotheses delivering results that can be easily replicated has meant marketing has concentrated on methodology, whereby the use of quantitative data and analytical tools has become more important than theory development. Consequently the discipline has focused on methods of theory verification with very little predictive value at the expense of theory generation.

However, the depiction of science as outlined above is a very traditional view. It will be argued that the natural sciences have moved on from this view and now encompass a much broader view of science (Delanty, 1997). As noted by Anderson (1983) empiricism has been abandoned by contemporary philosophy for at least the last four decades. It will also be argued that the debate is not about whether marketing is a science or an art but more a question about how marketing knowledge can be built and developed. 'Caught up in this academic version of trivial pursuit, marketing scholars and research practitioners have ignored overwhelming evidence that marketing is not a science!' (Anderson, 1994 p. 14). To substantiate this statement it is necessary to explore the concept of science in more depth. 
Science is a codified knowledge, based on certainty and reliability, so it is different from other systems of belief such as folklore, myth and religion. To obtain scientific knowledge it is necessary to suspend belief, that is, beliefs obtained prior to undertaking the research need to be suspended. This can mean that a researcher can be filled with doubt and uncertainty, which need to be seen as virtues rather than vices.

Science can be viewed from two perspectives. It can be seen as an end product of an activity that contains a body of knowledge, for example physics, or it can be viewed as an activity, that is, a particular type of method that is utilised to obtain knowledge. All sciences are about the acquisition of knowledge but not all knowledge can qualify as scientific knowledge. Scientific knowledge starts from the known to explore the unknown with a recognisable method that has its main constructs the notions of reproduction and verification (Razzaque, 1998).

Buzzell (1963) classified science as containing a systematic body of knowledge with central theories and general principles. '.. a classified and systematized body of knowledge...organised around one or more central theories and a number of general principles...usually expressed in quantitative terms...knowledge which permits the prediction and, under some circumstances, the control of future events' (Buzzell, 1963 p. 33). On the other hand, Hunt (1976) argues for a more liberal definition. First, it must contain a distinct subject matter. Second, there must be some underlying uniformities in the phenomena being studied, and third, the scientific method must be employed. 
A major feature of science is its ability to explain and predict (Chalmers, 1982). To obtain prediction generalizations need to be generated and this necessitates a move from singular statements to universal statements or general laws. To achieve this inductive reasoning is involved as the premises need to be settled by experience. Therefore the role of observation becomes important.

However, it needs to be noted that induction too has its problems. Bertrand Russell tells the story of the inductivist turkey who predicts that each day, based on past experience, he will get fed, but one day the turkey is plucked for dinner! The point is that one cannot use past experience to justify a prediction. Also, one cannot use past applications, which is induction, to justify induction as this leads to a circular argument. In other words, justifying induction on the grounds that it has worked before is itself an inductive argument, which makes the argument circular.

Also, there is the problem as to what counts as a large number of observations, or sufficient number of observations? How many observations are needed to make a statement true? This leads to a retreat to probability. In other words, scientific knowledge represents knowledge that is probably true, with generalisations being probably true as well.

It would seem that naïve inductivism will not help us to build marketing knowledge. To progress it is necessary to explore in more detail the role of observation. To begin, the following two assumptions are made. First, science begins with observation, and second, observation provides a secure base from which knowledge can be derived. What counts as an observation? 
The inductivist assumes that a one-to-one correspondence exists between an object and the image that is received by the retina. However, people do not have identical perceptual experiences therefore the notion of correspondence can be questioned. Also, there is a difference between perceptual experiences and observation statements. They need to be seen as two different things. To move from a perception to an observation statement, some type of theory is used. This means that theory of some kind must precede all observation statements. Observations statements utilise a language, which in turn has theoretical foundations. Consequently, observation statements are as fallible as the theories that they are based upon. They will be as precise as the theoretical conceptual framework that is used to express the observation. Therefore, it would seem that all observations are theory dependent. This means that it is not possible to make an observation without it being dependent on some theory, even if the theory is naïve or not very robust (Chalmers, 1982).

\section{Positivism}

The majority of marketing academics are positivists, in some sense of the word. They base their research on a realist theory by assuming that there is a real world out there to be observed and measured by the use of scientific methods thus gaining objective knowledge about the world. The label 'positivism' refers to a number of different research approaches such as logical positivism, logical empiricism, modern empiricism, foundationalism and objectivism. Ontologically, this group sees reality as being single and existing independently and so can be viewed objectively. Consequently, the phenomena being studied can be measured accurately. The overriding goal is explanation which entails predictions therefore researchers are 
looking for causal links. Understanding only comes after a systematic explanation.

Epistemologically the positivists attempt to generate general abstract laws that can be applied across a wide range of phenomena. To achieve these laws the positivist applies a strict methodology based on a scientific protocol with an explicit research design. This means a step by step approach is undertaken starting with a literature review, followed by a definition of the dependent and independent variables, statement of hypotheses, a demonstration of reliability and generalizability, and finally the establishment of statistical significance. Following such a protocol means that evaluative criteria can be easily applied.

'A positivistic naturalism assumes a natural order that obeys Hume's account of causality. There is no "metaphysical" natural necessity, but there are natural laws revealed through the constant conjunctions of observed events. The implicit ontology is empirical and binds reality to experience: anything that is observable is real, and anything that is real is observable. The epistemology asserts that theories should be appraised by observation and places trust in empiricism as a source of "factual" knowledge unadulterated by values or ulterior motives' (Jackson, 1995 p. 763).

The beginning of this movement can be traced to the Logical Positivists. The positivist method relies solely on observables so consequently avoids metaphysics. For a logical positivist, metaphysics are nonsense statements which are either reducible to empirically testable statements or by an appeal to logic. Also, ethical statements and value judgements were seen as in the same light so were nonsense statements. According to Hunt (1991), the positivists had four main objectives. 'The objectives of the logical positivists were to (1) help science make sense of the indeterministic nature of quantum mechanics, (2) help science avoid another Newtonian debacle, (3) help draw together or "unify" the various scientific disciplines, and (4) effect a rapprochement between science and the discipline of philosophy' (Hunt, 1991 p. 33). 
Logical positivists held that all meaningful statements were either verifiable concerning some sense data or were analytic statements based on mathematics or logic. They maintained a distinction between synthetic statements which were based on empirical data, that is sense data, and analytic statements which were self-evident. They did not believe that concepts were theory dependent, nor the idea that the history of science had scientific revolutions. The positivists thought that probabilistic prediction was fitting for science, so any theories must be seen in the light of calculation instruments for making predictions and nothing more.

In terms of unobservables, for example protons, the logical positivists believed that there were simply constructs introduced to explain how observables behave and therefore did not have an existence of their own.

There have been many criticisms of logical positivism but the main thrust came from Quine (1953) in his essay 'Two Dogmas of Empiricism' where he questioned the analytic/synthetic distinction, which is a central tenet of positivism. Whereas analytic statements are based on meaning, for example, all bachelors are unmarried, synthetic statements are based on fact. However, after analysing the term 'analytic' Quine argues that it is difficult to define so the distinction lacks meaning. Quine also questions the reductionism inherent in positivism, where meaning comes from the logical construction of terms based on experience. The positivists thought they could evaluate the truth value of each statement on its own, whereas Quine argues for a holistic approach because all statements are inter-connected. This being the case no statements are immune from revision, not even analytic statements. 
'The dogma of empiricism survives in the supposition that each statement, taken in isolation from its fellows, can admit of confirmation or infirmation at all. My counter suggestion, issuing essentially from Carnap's doctrine of the physical world in the Aufbau, is that our statements about the external world face the tribunal of sense experience not individually but only as a corporate body' (Quine, 1969 p. 135).

Quine's stance is that of a pragmatist. He believes that people make choices within a framework to suit their endeavours. 'Each man is given a scientific heritage plus a continuing barrage of sensory stimulation; and the considerations which guide him in warping his scientific heritage to fit his continuing sensory promptings are, where rational, pragmatic' (Quine, 1969 p. 139).

Despite the criticism of positivism, as Johan Arndt (1985) has noted, 'Marketing has been dominated by the logical empiricist paradigm stressing rationality, objectivity, and measurement.' Logical empiricism as a research programme emerged from logical positivism where verifiability was substituted with the testability principle. To be cognitively meaningful all statements had to be observationally testable. However, the logical empiricists continued to support Humean scepticism and rejected realism with respect to theories, laws and explanations. They too thought that the concept of 'causality' was a metaphysical concept, and therefore inappropriate.

Over and above Quine's criticism of positivism, Morgan (1983) points out that there are two important challenges to empiricism. One is the belief that a research protocol is objective, in the sense that it is devoid of any social and historical contexts. It is a myth that the researcher is being objective, which will be argued later in the chapter. The second criticism is that one cannot argue for the evaluation 
process to be based on the technical expertise of a particular method, be it positivist, interpretive, or action-oriented. As it was made clear in the previous chapter, the worth of any paradigm has to be justified on criteria that are relevant to the purpose. To build marketing knowledge different methods can be used to gather information so that creative research can be generated. This being the case, then Feyerabend (1988) is right in saying, with reference to methodology, that 'anything goes'. Therefore, the discussion goes beyond method, as there is no foundational approach.

Empiricist ontology limits objects of knowledge only to the observable and consequently, measurable, so concepts such as 'power' and 'social relations' cannot be taken into account (Marsden, 1993). Therefore, any explanation arises only from what is observable, which leads to a theory/practice dichotomy whereby theory is a description of external reality rather than being part of that reality.

'Science is a social and historical enterprise, and its knowledge-products can be affected as much by sociological factors as by purely 'cognitive' or ‘empirical considerations' (Anderson, 1986, p. 156).

According to Kuhn, theories fit into existing paradigms and become part of the extant knowledge, whereas those that don't fit are rejected. But it would seem that one reason such theories are rejected is not because of the content of the theory but how the theory was derived, that is, the process or methodology. Therefore methodology becomes the important yardstick with the consequence that the discipline can be isolated, narrow and unidimensional (Arndt, 1985). Also, such an approach means that the production of knowledge becomes the raison d'etre of the discipline whereby the preoccupation is centred on methodological issues and problems, instead of being more reflective and raising legitimate theoretical or conceptual concerns. 
'The use of social science as a technique for generating knowledge to help sustain, develop, or change society, together with the fact that it is conducted and evaluated within the context of bureaucratic structures, often tends to squeeze out the role of doubt in favour of a productionoriented mentality that emphasizes the importance of achieving significant, useful results. These considerations tend to favour an approach that is basically affirmational, in that the scientist is ultimately concerned with producing findings that add to a stack of knowledge by identifying hypotheses, insights, or explanations that can command some degree of empirical support, however small' (Morgan, 1983 p. 384).

Also one of the limitations of the dominant paradigm within the marketing discipline is that it has become narrow and limiting (Arndt, 1985). This in turn has meant that marketing has become ideological (Saren, 2000), in the sense that it is a doctrine based on managerialism and late capitalism. The problem with such an ideology is that the basic tenet of marketing, namely the marketing concept, is taken as an unquestionable given. It is only recently that marketing academics (Saren, 2000) are starting to question marketing's role in perpetuating consumerism.

\section{Scientism}

The chapter so far has outlined how contemporary marketing is based on what can be viewed as traditional science, which has been called "Story book" science (Zaltman, 1982) or "Fairy tale" science (Mitroff, 1972). Also the philosophical foundations of such an approach, namely empiricism were also outlined and discussed. In addition to the above, the concept of scientism needs to be explored and shown how it relates to the dominant paradigm.

Scientism makes the claim that scientific procedures based on the natural sciences, using their methods and imagery, are applicable to all areas of knowledge generation in the social world. In this respect, scientism can be seen as the pseudo emulation of 
'real' science by the social sciences. Although the term has many meanings, it is about the production of knowledge where disputes are settled by deciding whether the scientific method has been applied appropriately (Willmott, 1999). Therefore, any questions or concerns about the research that has been undertaken are kept at a methodological level. Consequently, assumptions at an ontological and epistemological level are blindly accepted or altogether ignored. 'With few exceptions, the revolution in philosophical thinking about science has gone unnoticed in marketing' (Peter, 1982 p. 11).

By adopting scientism as an approach to conducting research the overall task becomes the development of measurement instruments so as to predict human behaviour, with only a minimal appreciation or exploration of the historical and political context of the 'objects' being studied. This approach is perhaps privileging science in an uncritical fashion.

The belief underlying scientism can be attributed to positivism (Bernstein, 1983). The positivists believe that the natural and social sciences utilise the same methodology and measure independent facts, which exist in a single reality. To make this assertion is to believe there is an ontological continuity between the natural and the social world. For them reality is easily comprehended and contains discrete elements, which can be known and categorised. Therefore, methods used in the natural science such as experiments, quantifications, and hypothesis testing are deemed to be transferable to the sphere of the social sciences. Phenomena are viewed as historical events taking place sequentially in time and space and so causal determinants can be discovered. 
Critics of positivism believe that the same method as used in natural science is inappropriate for the social sciences because, by treating subjects as independent objects, they ignore the fact that the subjects being studied can think and act upon problems under scrutiny, thereby interfering with the data (Perry, Riege \& Brown, 1999), commonly called the 'Hawthorne Effect'.

Notwithstanding the influence of positivism on scientism, conceptions of science or the scientific method - have been idealised in our society resulting in it being granted with a privileged position in the generation of knowledge (Brown, 1992). Rorty (1989) sees scientists as the High Priests of modern society. It is to scientists that society turns to when problems arise in the community that need solving. It is the conception of science as dealing with the hard objective truth that society clings to, with the implicit understanding that there is a correspondence to reality. Consequently, so-called objective truth is accepted as the right explanation.

Yearley (1988) has given three reasons for the dominance of science in our culture. First, science has transformed our understanding of the natural and social worlds by the use of creative problem solving, coupled with technical innovation, leading to new products and new solutions. Second, science has shown considerable flexibility in being able to muster institutional resources with the result that it has been able to enrol allies to gain acceptance and momentum. Finally, science has proved to be very adaptable to a variety of legitimizing roles involved in the political arena and also in terms of economic growth. According to Yearley the above three reasons have led to science having a privileged status in modern society. 
Such idealisation of science in our society can be seen by our idolisation of medical research, engineering, the space programme and technology in general. These 'ideals' are icons of progress and are thought to have expertise which is superior to common sense. The idolisation of the scientific approach has meant that it has become universally accepted as the only approach to knowledge generation, which in turn has led to the domination by the scientific approach to areas outside of the natural sciences, leading to a type of colonisation. An alternative of such a view, as has been outlined, is that of accepting different paradigms whereby the generation of knowledge becomes a matter of subjective taste.

There are a number of problems associated with scientism, that is, the adoption of scientific methods for social science problems. The first is that the social sciences differ with respect to human action and that action can sometimes be influenced by the ideas of social scientists. Also, scientism does not take into account the social construction of such behaviour. It does not make sense to say that a mental thought has an existence on its own. How is it possible to make sense of the social world which involves actors? It would seem that a social activity could not exist independently of a mind or human agency. This is because intentions are involved. It is not just about functions. For example, would a credit card exist without it having an intention, that is for it to being used in a certain way, rather than just a material object, that is, a piece of plastic (Yankelovich, 1991).

Another problem is the narrow focus of scientism. Alfred North Whitehead was a philosopher who embraced the scientific method, but had misgivings about the 
severely limited aspect of reality that science focused its attention upon.

Yankelovich uses the term 'specialised searchlights' to outline the idea of sciences' narrow scope (Yankelovich, 1991). He believed that the adoption of the scientific method has led to specialisation and the generation of knowledge of a narrow, specific area, without taking into account the wider social aspects.

An additional problem with scientism, according to Yankelovich (1991), is that information becomes equivalent to a form of knowledge and opinion does not equal knowledge, consequently when making decisions information is preferred over opinion. Good information is seen as the key to decision making so the more facts the better the decision. When such facts are lacking then judgement is used as a fall back position to fill the vacuum. However, judgement is not a substitute but an independent variable and consequently plays a different role. Information is not reducible to judgement, or vice versa. All large marketing decisions involve both factual and judgemental elements, but good judgement cannot be assumed. Also facts obtained using solely the scientific approach is based on a distorted image of science.

'But philosophy and science itself teach us that the image of science as an objectivist discipline is badly distorted. Of course, facts and the theories that generate them are important to science. But they are not the only modes of knowing what science embraces. The "official" picture of how scientists work and the reality diverge sharply from one another. The great scientists are not advocates of so-called scientism, or objectivism, nor do they practice it' (Yankelovich 1991, p. 202).

In what Yankelovich (1991) calls a society with a 'culture of technical control', where objectivism is the philosophical doctrine ${ }^{10}$, there are some negative aspects,

\footnotetext{
${ }^{10}$ As a legacy of positivism, objectivism is a view that believes there are hard facts out in the world waiting to be discovered. It is seen as the only genuine method of knowing being based on
} 
such as the 'pollution of the planet, widespread alienation, spiritual superficiality and the failure of the community' (Yankelovich, 1991).

Whilst the problems of adopting scientism with the social sciences have been outlined, it is apposite to discuss the issues that are particular in marketing that negate scientism being adopted as a research approach.

According to Willmott (1999) marketing is presented with an unusual paradox. On the one hand marketing is viewed positively in both the public and private sectors, not only for products but also services, as well as including the not-for-profit sector. Over the last two decades the demand for marketing expertise in organisations has risen dramatically. However, according to some authors (Brown, 1995; Willmott, 1999; Hackley, 2001) marketing as an academic discipline is viewed with scepticism and suspicion and has not attained full respect by other disciplines within the commerce faculty, let alone the wider university community.

Marketing has responded by attempting to emulate scientific procedures in order to gain respectability. An additional approach has been to extend the scope of the discipline by researching new areas, such as tourism, leisure sector, and political marketing. This is a type of colonization, which can be seen as academic imperialism (Willmott, 1999). The above two responses have led to a disinclination to reflect more critically about knowledge development in marketing and also issues pertaining to the relation of power.

'Each stratagem for gaining greater recognition and credibility is seen to be problematical and, in some respects, to be counterproductive. A

verifiability and testability. From a philosophical point of view, objectivism is secured on a metaphysic based on a set of arbitrary biases. (Yankelovich 1991) 
scientistic philosophy of science inhibits an appreciation of the relationship between knowledge and power which, arguably, is important for developing a more rounded and coherent self-understanding of marketing discourse and practice; and those who seek to extend the domain of marketing expertise have not recognised the contradictory consequences of this imperialistic project' (Willmott, 1999 p. 207)

Also, scientism is adopted by marketing academics as it is easy and safe from the point of view of publication and career enhancement. If there is not a monopoly of one research programme over all other types, it becomes exceedingly important to be able to justify the research programme being used. However, if marketing is based on the natural sciences it is easy to justify as one adopts a powerful and logical methodology which is task-oriented based on technical expertise with outcomes being measured against design, collection and analysis. But gathering marketing knowledge is much more than being a mere technician.

The question needs to be asked whether it is possible for marketing theories to be developed that would meet scientific standards. The prima facie answer to this is that marketing deals with phenomena which are 'tightly coupled, nonlinear, and dynamic' (Buzzell, 1963) and therefore is unable to meet such standards. Also the marketing world is ontologically different from the world of the physical sciences. Unlike physics, there are no general laws to discover (Mathews, White \& Long, 1999). Different purposes of inquiry require different methods. It is argued that marketing is different from physics as the objects of study, that is, humans are selfconscious, language-using and information-processing animals whereby the researcher can influence the object being studied. 
It is also argued that with marketing research, complexity is ignored to make phenomena measurable. Also, unlike the natural sciences, marketing is value-laden and interactive with the social phenomena being studied which is always related to a given context. Following on from this, the natural sciences make the assumption that the phenomena they are studying are stable over time. As has been stated earlier, marketing activity takes place in a highly dynamic environment consequently conclusions reached at one point in time may not hold in the future. For example, the estimation of coupon redemption rates will vary across brands, and across segments. Therefore, studies in this area, where a controlled experiment was undertaken (Krishna \& Shoemaker, 1992), does not allow for accurate prediction.

\section{Scientific Realism}

As outlined in Chapter Two, there are a number of contenders to replace the methodology of the dominant paradigm in marketing. Hackley (1998) has promoted the social constructionist perspective for qualitative research in marketing, whilst Burton (2001) has discussed the merits of critical theory, and Easton (2002) has introduced critical realism as a replacement methodology. It is interesting to note that the above three approaches have very sparse literature in marketing, and it is also interesting that whilst the new approaches are clearly outlined, there is no philosophy of science justification as to why the approach should be adopted.

Excluding the work on postmodernism, which will be discussed in detail in the next chapter, the big debate in marketing has been between realism and relativism, which can be traced back to the Sophist-Plato debates. As has been shown, an empiricist account of science refuses to acknowledge the reality of non-empirical objects. 
Realism, on the other hand, does accept the existence of non-empirical entities.

According to Bhaskar (1978), positivism does not provide an accurate description of the processes undertaken by natural scientists. But the majority of marketing academics are empiricists in a very general sense. Hackley (2001) believes that the majority of marketing academics practice what may be called naïve realism. They base their research on a realist theory by assuming that there is a real world out there to be observed and measured by the use of scientific methods thus gaining objective knowledge about the world.

Hunt (1991), who may be called the defender of the faith, has attempted to overcome the paucity of philosophy of science discussion in the marketing literature by offering a defence of positivism in general and the need to be objective when undertaking marketing research.

Hunt (1990) argues for scientific realism to be adopted as the philosophy for marketing research and theory. There are several brands of scientific realism. The one being advocated here is where theories model the world in terms of its structure and causal properties, even if the world is not directly observable. Theories of science regarding non-observable entities, such as sub-atomic particles like quarks, do characterise actual properties of real things in the real world. For the Scientific Realist, scientific theories and their objects stand or fall together. They base their beliefs on inferential knowledge looking for causal explanation. In other words, they see the world as being causally explicable (Rescher 1987). Complex theoretical triangulation is used to explain complex entities, but it needs to be remembered that such entities only exist as current science envisions them to exist and at no stage does 
natural science yield a firm, final result. Science therefore needs to be seen as a dynamic process.

When it comes to seeking truth about nature, viz a viz, the laws of nature, it is a never-ending task as nature is cognitively inexhaustible (Rescher, 1987). Therefore there will always be more to learn about nature, so there is no truth with a capital ' $\mathrm{T}$ '. Because any knowledge gained is gained by an interaction of the mind with nature, the questions that are posed are limited by our conceptual abilities. Therefore the structure of the information processing of the mind will impact upon the object being investigated.

However, Scientific Realism has its critics. The relativists claim that traditional empiricism has certain problems (Fletcher, 1996). They argue that science is not value-free or politically neutral, and that data and the task of observation are influenced by the observer's theories. The reliance by marketers on the statistical significance of tests to determine whether the data supports hypotheses is suspect. Can such tests give straight yes or no answers? Is it reasonable to suggest that an effect at the $p=.049$ level is significant whereas a result at the $p=.051$ level is not significant? Also, what counts as an acceptable sample size in obtaining significant test results? The above questions highlight the problems with the statistical approach in marketing as there is no direct link between theory and data.

Also, it needs to be noted that inquiries involving induction only provide best estimates of any given phenomena at any give point in time. Consequently such findings are provisional and tentative and as outlined above, always open to revision 
and even to abandonment. Therefore, as there is no 'definitive truth', Scientific Realism is only a conceptual guide to give direction of an inquiry in terms of the thoughts and efforts required to obtain a result on any given piece of research.

Finally, one of the major criticisms of scientific realism is that it treats all phenomena as the same. 'The desire in contemporary scientific realism to represent all questions as one kind, as, in effect, empirical questions, and all justifications as of one kind, as empirical justifications, is simply another manifestation of the tendency to force a single representation on what is in no sense one unified phenomenon' (Putnam, 1997 p. 195).

Overall Hunt's position seems nothing more than a variation on a theme. In many respects his approach has reinforced realism as a dominant paradigm in marketing rather than changing the way research is undertaken.

\section{Relativism}

The alternative approach to realism is often seen as a relativist approach, which postulates that the world is created by the human mind, that the scientific method is no more reliable than other methods of gaining knowledge and the all epistemic assumptions are based on a social context. Since the mind makes the world, the world cannot serve as an external check on the beliefs held about the world. Human beings cannot transcend their language and culture so consequently it is impossible to obtain an independent view. Therefore the relativist has only an internal coherence as a form of test (Zinkham \& Hirschheim, 1992). 
Relativism is a general term covering a number of research approaches such as phenomenology, symbolic interactionism and hermeneutics. Relativists believe that reality is mentally perceived consequently reality is socially constructed. Therefore they deny that there is only one reality as different individuals or groups can have their own perspective. Hudson and Ozanne (1988) believes that the relativist needs a holistic approach as reality is made up of inter-linking systems which are dependent on each other for their meaning.

The goal of the relativist is to understand behaviour rather than predict it. A distinction needs to be made between explanation and understanding. The role of explanation is to seek causal links in the outside world, whereas with understanding the social world is understood from within the individual so the accent is on meaning rather than causes. With understanding the subjective meanings are all important. A core concept is that of vestehen, where the researcher places themselves in the role of the actor in an attempt to bridge the gap between the inner-world of the individual and the outer-world of actions. Obtaining an 'understanding' is more a process than an end product, so consequently interpretations are never complete. The basis for the understanding is the shared meanings that can be obtained by the active participation in a culture where the language and customs are studied. For the relativist it is this aspect that separates the social sciences from the physical sciences.

According to Hudson \& Ozanne (1988) the positivist holds a generalist view from which generalisations are possible. This is in contrast to the relativist who holds that any observation is at a particular point in time. In other words, observations are contingent on the situation and context. 
Also the relativists view the world as dynamic and ever changing so that it impossible to seek causal links. Unlike the positivists who adopt a determinist view point, the interpretivists adopt a voluntarist view of human behaviour. Research is an emergent process where new information is gained on an ongoing basis. 'The study is allowed to unfold with the assistance of informants. Ideas, meanings, questions, and data-collection techniques are cooperatively developed' (Hudson, 1988 p. 513).

One criticism of the relativist approach is that they rely on empathetic identification where a researcher relives another person's mental images, but such identification cannot be validated. Another criticism is that by focusing on the intentional actions of the individual there is no macroscopic understanding of society, in other words, no big picture of society is developed. A third criticism is that by intruding into the lives of the people being studied they may alter their behaviour which in turn may mean that the research is biased.

Not withstanding the above, in contrast to Hunt (1990), the relativists view truth claims as being based on a belief system within a particular context. Therefore the meaning of phenomena being studied is more important than the measurement of it. Consequently there is an emphasis on the ideologies and values of the social phenomena being studied.

Anderson (1986), one of the chief proponents of relativism takes a critical relativist stance when working in the area of consumer behaviour. Siegel (1988) argues that critical relativism is both internally inconsistent and self-defeating. The problem for 
the critical relativist is that if they utilize standards to evaluate the methods of a research programme they are open to an appeal in assessing what counts as a knowledge claim. 'To criticize and evaluate disciplinary knowledge claims, we must have standards that license those criticisms and evaluations' (Siegel, 1988 p. 131).

In contrast to the above approach is critical pluralism (Siegel, 1988) which allows for different methodologies to be criticized in terms of the purpose of the research but does not allow for ultimate evaluation. In other words, different approaches can be criticized in terms of what they are trying to achieve but it is impossible to categorically state that one approach is better than the other. This allows for alternative approaches to the study of marketing phenomena. Consequently a variety of legitimate perspectives and methodologies in research can be adopted but at the same time allowing for genuine criticality. It is this approach that was argued for in Chapter Two.

It would seem that the positions advocated in the marketing literature by the main proponents of relativism have paid little regard to the philosophical underpinnings of their position. 'Most of the R/C proponents present only a scant discussion of the philosophy without bringing out all its possible ramifications which are important for the doctrine's application in the field of marketing' (Razzaque, 1998 p. 7). In other words, one of the problems faced by relativists is that their methodologies in particular are difficult to put into practice.

Hunt (1992) believes the relativists are forced to become solipsists and that by abandoning the notion of truth they are committed do nihilism. However, the debate 
between the two positions is often thought as being independent and mutually

exclusive so that an either/or position is adopted. But in fact it needs to be seen as a

continuum with different positions being taken along the continuum.

The relativist position has been helped by the debate. With reference to the

discussion in Chapter Two about the hegemony of the functionalist paradigm and

Burrell and Morgan's (1979) reason for developing their framework, which was to

dilute this dominance, Brown notes that Hunt's continued attacks on relativism have

only helped the discussion.

'In his self-appointed role as the high priest of marketing scholarship, Hunt's apparent determination to condemn all marketing heretics to the flames of philosophical perdition merely served to focus attention on, and thereby helped legitimize, the relativist position. Conspiracy theorists might even conclude that the relativists engineered the confrontation, because it is only through conflict with the establishment that challenging groups can generate a sense of internal cohesion and shared purpose and succeed in attracting the support of the disenchanted, dispossessed and disenfranchised' (Brown, 1999 p. 44).

Both Anderson and Hunt did not see metaphysics as being scientific so the focus turned to a discussion of epistemology, which meant a shift from 'what is the world?' to 'how do we know about the world?' This meant the debate is about dichotomies, such as realism vs. idealism; positivism vs. anti-positivism; and empiricism vs. rationalism (Kavanagh, 1994).

However, as Saren (2000) has noted, the positions adopted by Hunt and Anderson have been modified over time and perhaps they are now closer together. This has meant that the debate has moved from the question of theory to the best approach to be adopted to obtain scientific knowledge. At best this has meant some epistemological debate, a lot of methodological considerations, but no ontological 
discussion. Ontology, which is a subset of metaphysics, is interested in understanding 'the very essence of the phenomena under investigation' (Burrell and Morgan, 1979 p. 1). From the above perspective, two opposing positions can be established, namely whether reality can be viewed as external to the perceiver or is dependent on individual consciousness. Therefore the question arises as to what entities one is prepared to posit in their world. This has ramifications for the subject/object debate, as well as questions about identity, which will be explored in Chapters Four and Five.

\section{Culture Theory}

The relativist position has been adopted by many consumer behaviour researchers, who take an interpretive approach to generate knowledge. This has implications for marketing management, not only because of the symbiotic relationship between the two schools, but also because of the wider scope of the discourse. As will be outlined in this section, consumer culture theory places an importance on the wider social context, instead of basis its modus operandi on manageralism (Hackley, 1999).

Shankar \& Patterson (2001), postulate that interpretivist consumer research (ICR) has developed over four distinct stages. The first took the form of a naturalistic inquiry, where the approach was based on a quasi-positivism paradigm. The thrust of this approach has been to legitimise interpretivist consumer research by the use of four criteria, namely credibility (to replace internal validity); dependability (as a substitute for reliability); transferability (to replace external validity); and 
confirmability (as a substitute for objectivity). ${ }^{11}$ According to Kennedy, Goolsby and Arnould (2003), the creditability of respondents can be determined by ascertaining whether the information received is trustworthy, and this can be determined by asking whether the conclusions make sense and whether they are plausible to the informants and stakeholders. This stage of ICR was an attempt to merge interpretive consumer research with positivism so as to gain acceptance within the academic community (Shankar and Patterson, 2001). As a consequence of this exercise the debate about what constituted good research was reduced to methodological issues. ${ }^{12}$

The second phase of Interpretivist Consumer Research $\left(\mathrm{ICR}_{2}\right)$ was centred on philosophical issues, as has been outlined in the preceding sections. In summary, the result of this endeavour was to threaten the strong hold of positivism, especially in the area of consumer behaviour. ' $\mathrm{ICR}_{2}$ and the work that stems from it, however, elevated academic discourse from methodology to the other paradigmal questions of ontology and epistemology and severely threatened the philosophical beliefs of positivists' (Shankar and Patterson, 2001 p. 486). The consequence of this debate was that interpretivists did not feel the need to justify their research approach to positivists, but rather to other interpretivist schools of thought.

The third stage of Interpretivist Consumer Research was based on advancing the empirical work of the different interpretivist schools. Three examples of

\footnotetext{
${ }^{11}$ For an additional insight refer to Hogg \& Maclaren (2008), who outline the approach interpretive authors take to convince their audiences of the soundness of their "theorized storylines". Their study used an analytical framework based on three dimensions, namely authenticity, plausibility and criticality, to analyse examples from consumer research.

${ }^{12}$ Refer to Chapter 2, page 61 regarding the problems with triangulation as a research protocol.
} 
interpretivist schools will now be explored. The first is ethnographic studies, which has the aim of observing behaviour as it happens in real time. This is in contrast to surveys that either asks about past behaviour or future intentions. 'Ethnographers observe everyday events, settings, interactions, conversations, and uses of objects over time and across specific cases' (Arnould and Wallendorf, 1984 p. 486).

According to Arnould and Wallendorf (1994), there are two general methods employed when undertaking an ethnographic study. The first is participant observation, whereby the researcher is allowed to observe complex behaviour over time, and as the observer becomes accepted into the group, he/she is able to gain access to "backstage" areas, where behaviours are rehearsed and scripted. The second method is that of non-participant observation whereby the researcher is not a member of the group being observed so the data collection is obtained from verified reports.

As Arnould and Wallendorf note (1994), unstructured interviews have been utilised in ethnographic studies, typically with individual respondents. The emphasis is on general enquiry rather than a highly specific structure. An example of an ethnographic is Schouten and McAlexander's (1995) study of the subculture of consumption, exploring how brands (in this case Harley Davidson) can be used as a basis for social interaction. The authors studied the interaction of motor cycle owners, identifying the social structure, dominant values, and symbolic behaviour of the bikers, and the relationship between the marketing institution and the subculture. 
According to Kennedy, Goolsby and Arnould (2003), the benefit of ethnographic research is that it allows for the generation of varying perspectives. 'As is typical of extended ethnographic research, the iterative and flexible research design encouraged exploration, enabling the emergence of additional research questions, unexpected data, and a theoretical understanding' Kennedy, Goolsby and Arnould 2003 p. 69.

Phenomenology is another school which has extensive literature. Stern, Thompson and Arnould (1998) use narrative as a means to understand cognitive and affective responses to brands, advertising, and interpersonal exchanges. They utilise freeform, consumer-driven text as an attempt to elicit the informant's perspective, rather than a directive interview that fulfils the expectations of the researcher. In other words, they are interested in the respondent's story without a structure imposed by the interviewer. 'That is, the interview technique facilitates the phenomenological study of lived meaning, defined as higher-order meanings captured by a single experience set in the context of personal history over time' (Stern, Thompson and Arnould, 1998 p. 198). An article by Goulding (2005) compares and contrasts grounded theory, ethnography and phenomenology, and suggests a number of contexts that the approaches could be beneficial within the broader marketing field. As she notes, the use of such methods is now widely accepted in the consumer research area, for example, the work by Thompson, Locander and Pollio (1990) has utilized a phenomenological approach in their study of the consumption experiences of married women, but the use of qualitative methods is not so readily accepted in mainstream marketing, viz. the marketing management school. 
Whilst the above three stages have consumption as their main interest, they are delineated by their methodology. Alongside the above approaches but sitting outside of mainstream consumer behaviour is the topic of consumer culture theory (CCT).

CCT is not a grand theory and does not claim to have any generalizable laws. However, there have been different interpretations regarding the significance of consumer culture in society. 'While there is consensus about the significance of consumer culture, its theoretical interpretation has been a contested terrain. Consumer culture has been interpreted in multifarious ways including consumption as a control mechanism, as environmentally harmful, as giving new meaning to life, and as having an emancipatory potential' Varman and Belk, 2008 p. 229). Consequently, as noted by Varman and Belk (2008), it would appear that consumer culture has multifarious effects and cannot be viewed in simplistic terms.

However, a central theme within CCT is that of consumption, linked with consumerism. Belk (1985), for instance, Belk (1985) explored the relationship between materialism and marketing. He identified in the extant literature on the subject of materialism four major issues, namely the question regarding whether it is a positive or negative trait; whether marketing creates or exacerbates materialism; the issue concerning materialism as egoistic and thereby excluding altruism and sharing behaviours; and the impact of materialism on interpersonal relationships, especially centred around the raising of children as material goods are often used as reinforces of desired behaviour. 
In his study Belk used three materialism scales to measure the constructs of possessiveness, non-generosity and envy, and found that these constructs when combined are correlated with the overall materialism scale. However, he did find that there were generational differences, and found that materialism as a dominant value lessens with age, although the magnitude of difference was not too significant.

Whilst the majority of marketing academic work within consumer behaviour is oriented towards macro-economic theory, cognitive psychology and quantitative modeling, CCT argues that researchers need to take into account the context of the situation, not as an end in itself, but as a means to generate new insights and theoretical constructs. The study of context means that the experiential, social and cultural dimensions need to be taken into account. Following on from this, the consequence is that qualitative methods serve the purpose of such studies, rather than experiments, surveys, or database modelling. However, CCT accepts methodological pluralism, with respect that the best method should be used to understand the data at hand.

'Consumer culture theory is organized around a core set of theoretical questions related to the relationships among consumers' personal and collective identities: the cultures created and embodied in the lived worlds of consumers: underlying experiences, processes and structures: and the nature and dynamics of the sociological categories through and across which these consumer culture dynamics are enacted and inflected' (Arnould and Thompson, 2005 p. 870). 
Arnould and Thompson (2005), caution that the adoption of a managerial perspective imposes a barrier to the investigation of consumption in a wider sociocultural context. They also are concerned that consumer behaviour is seen as a sub-discipline of marketing rather than an autonomous discipline in its own right.

The economic exchange model, used as the basis for the marketing management school, can be contrasted with social exchange. Both concepts have the notion of reciprocity as a key element. However, the economic model is based on the belief that exchange takes place in a rational, objective basis. Social exchange, on the other hand, utilises the concept of symbolic value to underscore the idea of reciprocity. In understanding such exchange, a bond of goodwill and social indebtedness between the two parties is created. The love that we display towards certain consumer items, be it a car or a new coat, can often elicit self-less passion rather than instrumental acquisition based on some notion of materialism. This is explained by Campbell (1987) who argues that consumerism is based on romanticism, that is, the precedence of feelings over reason, rather than utilitarianism. With gift giving, for example, gifts are valued for their symbolic worth rather than their economic value (Belk \& Coon, 1993).

Central to consumer theory is the exploration of symbolic meanings encoded in the marketing arena, such as advertisements and retail settings, which are internalised by consumers to develop their identity and lifestyle goals. Belk (1988) argues that possessions are part of who we are. Basically, we are what we have, that is, our possessions are central to our identity. The relation between possessions and self, helps one to understand the notion of extended self. The concept 'extended self' is 
used by Belk as a metaphor which combines the concept of self (the "me") with the concept of possessions (the "mine"). As these two concepts are fused into one, it becomes difficult to draw a distinction between the two concepts, especially as what is "mine" does not only include material objects but also intangible aspects, such as fame, places, poverty, and groups of people, for example, children.

Understanding this perspective allows marketers to understand the part consumer behaviour plays in understanding human being's existence. 'If seems an inescapable fact of modern life that we learn, define, and remind ourselves of who we are by our possessions' (Belk, 1998 p. 160).

According to Shankar and Patterson (2001) the final stage has seen a challenge by postmodernism to the interpretive consumer research stream that was developed in the first three stages, which has signalled a significant difference as the first three stages were based on a realist ontology, whereas postmodernism takes a relativist position. However, before the topic of postmodernism is explored in the next chapter, it is apposite to evaluate the theory of marketing management by using a Lakatosian framework.

Given the wider scope of consumer behaviour it is argued, along with Belk (1998), that the subject be treated as a separate discipline. This would allow other areas outside of marketing that critique consumerism and marketing's role in promoting western capitalist ideology to be included in the research agenda. Three important works are worth mentioning as examples. First, George Ritzer's (1996) 'The McDonaldization of society: An Investigation into the Changing Character of 
Contemporary Social Life' referring to shopping malls as 'cathedrals of consumption'. Second, Colin Campbell's (1987) book 'The Romantic Ethic and the Spirit of Modern Consumerism' indicates how romanticism is linked with consumerism and that it is through consumption that the 'self' is able to gain expression. The third book is Arlie Hochschild's (1983) 'The Managed Heart: Commercialization of Human Feeling', which is a study of the adaptation of emotions for commercial purposes, for example, airline cabin crew.

This chapter has outlined issues surrounding the dominant paradigm in marketing management, and discussed issues regarding positivism, scientism, scientific realism and relativism, from a modernist perspective. The stages of interpretivist consumer research have been explored and it is argued that the school of consumer behaviour, with a wider scope to include consumerism and consumption, should be treated as a separate discipline. Before the chapter on postmodernism is examined, it is apposite to review the marketing management discipline using a Lakatosian framework.

\section{Lakatos}

Marketing knowledge is based on observations which are formulated in the language of some theory. Consequently, such an observation statement's precision or accuracy will in part be dependent on the precision of the language used to frame the statement. Therefore, marketing knowledge will be based on some theoretical guidelines or paradigm with underlying assumptions. These paradigms build up a body of knowledge based on a research programme. Has marketing spelt out a coherent research programme to guide future research? 
Understanding that an on-going research programme is usually inspired by a theoretical position, it is important that the dynamics of research are grappled with, so that it is possible to evaluate such programmes. Lakatos (1970) provides a way for such an evaluation to tentatively take place. His research programmes have four elements, namely: a hard core, consisting of unchanged, privileged knowledge; a negative heuristic, which forbids knowledge from the core being directly challenged; a protective belt of auxiliary hypotheses that bear the brunt of tests that get adjusted or re-adjusted in an effort to defend the core; and a positive heuristic that guides the production of positive theories within the programme.

However, how can research programmes be evaluated? This presents an intriguing problem. As it has been shown, no observation is theory-independent, so science involves a process of interaction. Berkley (1709) showed that an object gains its objectivity only by being observed, and that the notion of objectivity is a property which stems from the observer. Research can be viewed as an engagement between the researcher and the researched. However, it is possible to study phenomenon in different ways, using different frameworks and frames of reference. Therefore, is it possible to stand outside and evaluate a research programme? This can be difficult as a programme contains certain assumptions and therefore cannot be judged from within the programme, as this would lead to circularity. Consequently, a model is needed to evaluate, in some sense, a research programme. A good place to start is with reflective discourse whereby the merits and significance of different points of view are made explicit. 
It is necessary to explore the distinctive ways research is undertaken. It is not just a question of the methodology that is used in any give programme. It is more than just that, as the assumptions behind the methodology need to be understood. In undertaking a reflective discourse comparing different research programmes the task is to look for consistency and coherence in a research programme.

Lakatos was more interested in research programmes that build a schemata of knowledge, than in assessing the merits or otherwise of individual paradigms. $\mathrm{He}$ took Kuhn's argument a step further, as he believed whole research programmes can be falsified, which occurs when alternatives are available. The history of science shows that progressive programmes replace less adequate ones, but all programmes are important to the progress of science.

Lakatos did not believe that paradigm incommensurability was the issue. For him there was no "instant rationality" but that scientific endeavour was time dependent. A piece of research may be dismissed at one period of time but later on be accepted by the scientific community, and vice versa. He did not believe the issue was about one piece of scientific research nor what paradigm the research belonged to. What was of paramount importance for Lakatos was the programme of scientific research.

Unlike Kuhn, Lakatos did not believe that a revolution was needed for a programme to be replaced, but that a budding research programme could operate alongside the dominant programme. Lakatos attempted to propose a synthesis between Popper's falsificationism and Kuhn's sociology of science. Lakatos called his approach 
'sophisticated falsification', which needs to be compared with 'naïve falsification', an approach that evaluates a scientific theory in isolation (Fawundu, 1991).

For Lakatos, research programmes will be progressive or degenerative depending on whether they succeed in leading or whether they persistently fail to lead to the discovery of novel phenomena.

Scientific Research Programmes (SRP) are theoretically progressive if they produce successive formulations which contain 'excess empirical content' in the sense that they predict some novel, hitherto unexpected fact. The programme becomes empirically progressive if this empirical content is corroborated. A research programme is said to be degenerative if endless ad hoc adjustments are made so as to accommodate new facts as they become available. To make the call in appraising a research programme it needs to be done over a period of time. There is no "instant rationality" in the sense that there is no means to judge current events. Something that may be seen at the present point in time to be of little significance can at a later stage be seen as revolutionary.

Lakatos developed operational criteria for appraising research programmes. These criteria are to be used to determine two sorts of research programmes, what he calls 'intra problemshifts' and 'inter programme problemshifts'. The former problemshifts occur within a research programme whereby modifications are made to the protective belt of auxiliary hypotheses, whereas the latter are types of problemshifts when elements of the hard core are rewritten. If these problemshifts are ad hoc then the research programme is deemed to be degenerative. 
For Lakatos, scientific enquiry was like a game with evolving rules. There are the hard core rules that could not be changed as the game would lose its identity. For instance, if you were to change the game of rugby by not having goal posts and playing with a round ball instead of an oval ball then the game would be completely changed and wouldn't be seen as rugby. However, around the hard core is a protective belt where the rules are expandable, for example, lifting in a line-out, and these rules change over time in light of experience. The change is directed by a "positive heuristic".

Any changes to the core can be seen as either progressive or degenerative. But what counts as 'progressive' or 'degenerative'? There are no operational indicators available (Vasquez, 1997) but the key characteristics can be identified. As a research programme increasingly takes on ad hoc explanations without adding any new theoretical facts, the programme can be deemed as being degenerative.

If it is not possible to modify the hard core a new research programme needs to be launched. Lakatos, along with Popper and Feyerabend, and against Kuhn, agree that science must contain competing research programmes (Musgrave, 1978).

The protective belt contains the flexible parts of the scientific research programme whereby the hard core is combined with auxiliary assumptions to form specific testable theories. Just because the hard core is at risk does not necessarily mean the demise of the programme as the job of the protective belt is to dissipate any attacks. If the protective belt fails then the research programme is in serious difficulty. 
Lakatos distinguishes three ad hoc types. The first is where the theory depicts no novel consequences. The second is where the theory has not been corroborated. The third is where the theory is obtained from its predecessor through a modification of the auxiliary hypotheses that are not in agreement with the positive heuristic of the programme.

Following on from the above, inter-programme problemshifts must satisfy first and second ad hoc types, whereas intra-programme problemshifts must satisfy the third ad hoc type.

There is considerable debate regarding the criteria that need to be used to decide what would count as a novel act. Elman (1997) believes that novel acts can be grouped in to four categories. The first is what could be called 'strict temporal novelty'. The second developed by Lakatos is that of the novelty of a new interpretation. The third category developed by Worral (1978) from a formulation by Zahor and Lakatos is that of heuristic novelty. The final category is Musgrave's novelty of background theory. Elman prefers the third category. 'One can't use the same fact twice: once in the construction of a theory and then again in its support' (Elman \&Elman, 1997 p. 924).

The role of theory is to depict the organisation of a research sphere. It follows then that a sphere can be organised in an infinite number of ways, leading to endless different theories. Positivists believe that reality can be directly observed so there is no need for theory. There is a direct correspondence between what is observed and 
reality. A modified version of positivism is that theory is one step back from reality, can be obtained by induction and is relatively easy to test.

But facts are theory-laden, therefore, theory is not a mirror of reality but an instrument used to explain a certain part of reality. Consequently, the truth statement depicting a reality can never be measured. For Lakatos all theories are born false, which is essentially a Hegelian view. As all theories are incomplete they are inherently fallible, consequently they are false. This means that false theories only increase knowledge. A programme is seen to be progressive if it leads to new knowledge.

Lakatos rejected Popper's view of theory falsification. (Blaug, 1976) Popper advocated a bold commitment to falsifiable predictions whereby theories are abandoned if they fail to survive refutations made against their predictions. Such an approach involves a continuous revolution as all theories are always open to rejection and/or modifications. This is a normative methodology as it prescribes sound practice in science.

There are two problems with falsification. The first is that if one is to prove something true, then you need to prove something is false. 'Among natural scientists, falsification is a little used method' (Waltz, 1997 p. 914). The second is that theories are evaluated with a ceteris paribus clause implied so it can never be proved that the theory holds. This means that theories are mere idealisations. 
Lakatos argued against Popper (Vasquez, 1997) as he believed that no single theory could be falsified as it was always possible to add auxiliary propositions. A set of theories share assumptions about the way the world is viewed. Therefore, one theory being replaced within a paradigm, in an incremental fashion, does not lead to a rejection of the fundamental assumptions.

Lakatos' approach is a compromise between the 'aggressive methodology' proposed by Popper and the 'defensive methodology' postulated by Kuhn. (Blaug, 1976) Lakatos is not about laying down standard procedures for scientific research but he is concerned with the logical appraisal of a normative problem regarding the criteria for assessing scientific progress.

According to Lakatos, the merit of a theory should be judged by the degree that it influences the birth and growth of a research programme. For Lakatos a theory can only be rejected in the context of the entire programme. However, this does not mean that a theory would not be rejected on the basis of Popper's falsification. The status of the theory within the programme would need to be taken into account before a decision could be made.

What Lakatos has done, following on from Popper, with the notion of heuristic power is to take the falsificationist account of what it is to develop a theory and to defend it against criticism (Musgrave, 1978). Lakatos's approach provides a methodology to evaluate a programme as a whole, and therefore compare one programme with another research programme. But the evaluation does not give advice to individual researchers about what programme to work on. It may be that 
some scientists should continue to work on a programme in an attempt to try and improve it, and on the other hand, it may be wise for some scientists to switch depending on the idiosyncratic factors the individual may face. Advice regarding a research programme can only be given to the research community as a whole. However, it would seem strange for an academic community to persist wholesale with a degenerating programme.

However, despite the above misgivings, it is beneficial to see if Lakatos's methodology can be applied to marketing. As an example, Blaug quotes the work of Latsis as an example of the framework being applied to classical economics.

"Latsis argues convincingly that theories of perfect and imperfect competition may be considered together as forming part of the same neoclassical research programme in business behaviour with one indentifiable 'hard core', one 'protective belt', and one 'positive heuristic'. The 'hard core' is made up of (1) profit-maximisation, (2) perfect knowledge, (3) independence of decisions, and (4) perfect markets. The 'protective belt' includes several auxiliary assumptions: (1) product homogeneity, (2) large numbers, and (3) free entry and exit'. The 'positive heuristic' consists of 'the analysis of equilibrium conditions as well as comparative statics' (Blaug, 1976, p. 175).

If the hard core comprises tenets concerning buyer behaviour, seller capabilities, the institutions to support such capabilities and the environment in which they operate, as Leong (1985) suggests, it would seem that the protective belt is hard to define, especially if it comprises competing theories, for example, information processing models compared to behaviourism. Also, middle range theories are hard to define due to the amount of theory borrowing that takes place in the marketing discipline. As Bradley (1999) has noted with regard to neo-classical economics, perhaps marketing is characterised more as a set of techniques for handling data than a 
cohesive research paradigm. He then goes on to say that marketing is not a science in the Lakatosian sense.

Whilst it is difficult to apply Lakatos framework rigidly to marketing's dominant research programme, there is doubt whether it could be called a progressive research programme. The arguments outlined in this chapter would support the view that marketing management will never develop a progressive research programme. However, as will be argued later in the thesis, the idea of there being a dominant paradigm in marketing management in a foundational sense, that is, there is a 'right' paradigm with its own specific language, will be argued against. To pursue this line of argument the topic of postmodernism will be explored in-depth in the next chapter.

\section{Conclusion}

The purpose of this chapter has been to explore the dominant paradigm as used by the major school in marketing, namely the marketing management school. The emphasis in most marketing text books is that of marketing management which has a central tenet of profit maximisation by manipulating the marketing mix, comprising product, place, promotion and price. This is commonly referred to as the four 'Ps" of marketing. The philosophical framework utilised is primarily empiricism with a heavy reliance on the so-called scientific method. However this has raised a number of concerns. First, it can be seen that social factors are linked to the production of all scientific knowledge, therefore it can hardly be called 'objective'. Also, research in any paradigm is motivated by the social milieu of the researchers, which means that academics primarily practice conformist behaviour. This is reinforced by the 
external gatekeepers. Another point is that $\mathrm{PhD}$ programmes typically have methodology courses on how to conduct research based on statistical programmes with validity, reliability and bias being the primary concern, without any concern about the philosophy of science issues. Consequently such candidates graduate with a narrow set of internalised criteria regarding 'acceptable' research and become easily institutionalised into their new academic appointment.

The chapter also indicated that any alternative to the current dominant paradigm, be it scientific realism or a form of relativism, is locked in at a methodological level and denies the opportunity for any theory building to take place. Also, theory borrowing does not provide great opportunities for marketing to develop as a discipline. The chapter also suggested that theory generation cannot be developed in marketing with an emphasis on empirical research, data analysis, and quantitative modelling.

After applying the Lakatos framework for evaluating marketing management there is doubt whether it can be claimed to be a progressive research programme. This means that marketing may not necessarily abandon the research programme but it is necessary to cast the net further and evaluate frameworks which are not based on a modernist conception, with its inherent dichotomies. The next chapter, Understanding Postmodernism, explores the topic of postmodernism and suggests that further exploration is needed before a framework is found which will allow marketing management knowledge generation, based on the linguistic turn in philosophy, to take place. 


\section{Chapter 4: Understanding Postmodernism}

\section{Introduction}

In Chapter One it was claimed that the marketing discipline should adopt the linguistic turn in modern philosophy. To justify this claim it was necessary to explore the philosophical underpinnings of marketing management. Chapter Two reviewed the paradigm concept and Kuhn's ideas regarding the way research changes direction within a discipline. Burrell and Morgan's framework was explored as an overview of the different paradigms available to researchers. However, the Deetz framework was favoured as it was based on discourse and allowed for other types of research programmes to take place. This was followed by a discussion of the problems of paradigm incommensurability. Chapter Three discussed the dominant paradigm in marketing, namely logical empiricism. Pitfalls of this approach were highlighted. Using an evaluative framework developed by Lakatos, it was seen that the discipline cannot be categorised as undertaking a progressive research programme.

Therefore, the purpose of this chapter is to explore the topic of postmodernism and determine whether it leads to an alternative research approach that will generate marketing management discourse based on the linguistic turn in philosophy.

Postmodernism is a extremely broad topic. From a philosophical perspective, it can be seen that Wittgenstein laid the foundation for relativism and post-modernism. According to Kavanagh (1994), postmodernism is an attempt to move away from anthropocentrism and the rationality of modernity. Whilst it can be claimed that it 
has failed to provide a more attractive alternative (Kavanagh, 1994; Brown, 1995) it haunts the social sciences in general but provides endless challenges, particularly for the field of marketing. It would appear that postmodernism is very close to poststructuralism so what is written about the former is equally applicable to the latter.

Postmodernism heralds a new philosophy which is an attack on positivism and calls for the complete abandonment of the entire intellectual culture of modernity and in particular Marxism (Delanty, 1997). The central ideas of postmodernism are that society should be interpreted as a text and the deconstruction of agency involves a shift in emphasis from structure to culture. Also due to its anti-foundational stance involving cultural relativism and plurality, there is no correct view.

Following on from this, the marketing discipline is in an intellectual crisis, as indicated in chapter one and this can be linked with postmodernism as per a crisis of representation whereby rationality, truth, and progress have been challenged. So will post-modernism deliver a basis for marketing knowledge to be built? To determine the answer to this question we first must explore postmodernism in more detail.

\section{Defining Postmodernism}

What is postmodernism? This is a hard question to answer as contributions to the literature about postmodernism come from many different disciplines. Consequently the terminology and perspectives are very varied (Firat, Dholakia, \& Venkatesh 1995). It has been suggested by Kilduff \& Mehra (1997) that for every postmodernist there is a postmodernism. Given this situation, problems with the definition of 'postmodernity' arise as the term can mean different things depending 
on the context in which the term is being used. Some see the term as an epoch, a state of mind, a condition or a school of thought (Schatzki, 1993).

In addition to the above problems, Rosenau (1992) claims that there are two types of postmodernists. The first are sceptical postmodernists who view the world as doomed, haunted by the immediacy of death, and the impossibility of truth, hence they view life as meaningless. The reaction to this angst ${ }^{13}$ is to emphasize gaiety whilst waiting for catastrophe to happen, so consequently there is an emphasis on play. The other type of postmodernist is that of the affirmative who base their beliefs on optimism. They are oriented towards process based on philosophical and ontological intellectual practice. This thesis is concerned with the affirmative type of postmodernism as it allows the possibility of building marketing knowledge. This will be explored in more detail in the next chapter.

However, further distinctions need to be made. Brown (1999) outlines three sorts of postmodernism. The first sort can be seen in the areas of art/design and architecture whereby it is asserted that there is no artistic orthodoxy or single dominant style to follow but a smorgasbord of choice. This leads to an eclectic ironic style, where different periods are interwoven into a creation or construction. The outcome of this trend is that high and low cultures are fused.

The second type of postmodernism is that of postmodernity whereby social and economic development are explored. Within this type certain trends can be observed such as the decline of organised religion, the demise of the nuclear family, the

\footnotetext{
13 The influence of Heidegger and Nietzsche on postmodern thought will be explored in the latter part of the chapter when discussing the work of Foucault
} 
proliferation of media and the increase of communication technologies. Also within this category is the rise of mass customization whereby niche markets are supplied by service-driven company cultures. This type of postmodernism is concerned with cultural analysis.

The third emphasis of postmodernism is that shared with post-structural thinkers with a common theme of textuality, narrative and discourse, and language. This type of postmodernism is exploring issues from a philosophical perspective that, in part, has arisen from the second type, that is, cultural analysis. This thesis is primarily concerned with the latter two types as they both pertain to the marketing discipline and thereby gives some clear insight to understanding the perimeters of marketing knowledge.

But before the area of cultural analysis and the philosophical implications of postmodernism can be explored it is necessary to make some general observations, and in particular understand the meaning of modernity, which postmodernism is rallying against.

\section{Modernity}

Postmodernism can be seen as an internal critique of modernity (Dallmayr, 1993).

The task is to reveal the inner complexity and ambiguity of modern consciousness and rationality. However, the meaning of postmodernism is predicated on the meaning of modernism so consequently to understand postmodernism it is necessary to understand modernism (Graham \& Doherty, 1992). 
There are different terms that refer to the modern period, each with its own meaning. The first is modernity which has an economic orientation and is used to refer to the transformation of life under capitalism, especially with reference to nineteenth century and twentieth century development of urbanisation due to the growth of cities and metropolises. Another term is that of modernisation, which refers to technological innovations in society. This in turn impacts upon the term of modernism, which is concerned with social developments in society and in particular, movements in arts, culture, and philosophy.

Modern development has been periodized into four segments (Graham \& Doherty, 1992). The first segment is that of the Enlightenment, which can be called the 'Age of Reason'. During this time the authority of the state and church was being undermined. Philosophers such as Locke, Pascal, and Descartes promulgated the ideas of rationality and science and that the notion of social progress of society was an important tenet. Rousseau (Flew, 1971) wrote about the increase in freedom and liberty due to an increase in knowledge gained by the populace. Overall, during this period there was a general feeling of optimism.

The second segment is post- 1850 whereby it was claimed that there were many ways to discover truth. Overall, during this period, the underlying theme was that of aestheticism built on both a scientific and cultural endeavour. Philosophers, artists, and scientists prominent during this period were Nietzsche, Marx, Lenin, Weber, Joyce, Manet, Einstein, and Picasso. Unlike the previous period, the general theme was one of scepticism and pessimism, especially with regard to industrial 
capitalism's constraint on the ideals of individual liberty and quality of life in general.

The third segment is that of the heroic interwar period. The main theme of this period was the search for a 'myth' to overcome the wounds of war and as a means to move towards progress and achievement. However, the need for a 'hero' led to the rise of Mussolini and also Stalin, whereby societal realism was underpinned by cultural and artistic endeavours leading to totalitarian regimes.

The final segment is the of the high modernism post-1945 period with the worldwide dominance of the United States of America, international capitalism, and global markets. The theme of the enlightenment with its appended feeling of optimism was revived based on an underlying faith of an authoritarian elite such as planners, architects, intellectuals who were employed in the service of maintaining the status quo. Consequently, modernism was usurped by the establishment, comprising international corporations and institutionalised power. Such a shift in modernism has meant that the thrust of the movement has been one of acceptance rather than a force to gain change. Modernism has ended-up being anti-modernist.

According to Graham \& Doherty(1992), one strand that runs through the four periods is that of tension between, on the one hand, change and mobility and on the other hand, the search for immutable and eternal truth within an ordered reality. The above tension explains the experimentation that took place in this period. However, within the last period the ordered reality has been realised with the 'American Dream' in the shape of western liberal democracy. Positivism has been the favoured 
methodology in social science with the seemingly 'objective' application of the scientific method, although there have been some dissenting voices such as structuralism, realism, behaviourism, and humanism. But more poignantly, all the above approaches have one theme in common and that is the search for underlying order in social science by way of a master narrative to embrace a universalistic understanding of society.

Within the modern period, the role of science is seen as value-neutral (Hollinger, 1994). This means the scientist is not involved in any moral or ethical issues regarding the value of their work to society. This is based on the 'is-ought' distinction, which comes from Hume who stated that no statement of value can be logically derived from factual premises. Therefore a number of dichotomies arise from this distinction, which the positivists built upon, such as the fact versus value distinction, the descriptive versus prescriptive dichotomy.

It needs to be remembered that one of the underlying premises of modernism is the ethos derived from the era of the enlightenment. The enlightenment period came to fruition in the $18^{\text {th }}$ century but it has considerable internal diversity. Nonetheless, there are some common themes, as follows: There is an emphasis on reason, so the concept of rationality is important. Second, empiricism as an epistemology is postulated, so there is an emphasis on observed, empirical facts. Third, is that the idea of the scientific method is put on a pedestal with its concomitant drive to isolate truth and to expand knowledge. Following on from the above is the idea of universalism, whereby general laws can be derived from reason and science. However, scepticism plays an important part as it asserted that all knowledge claims 
should be scrutinized. Finally, the concept of secularism is important so that all religious dogma and superstition is eliminated. All the above central themes are based on a core idea of progress.

In summary modernism can be characterised as having the following features. First, there is progress in thought and consequently we learn from the past. Second, there is a search for universal theories. Third, there is a tendency towards professionalism where there is a separation of disciplines. Also there is increasing specialization in intellectual and functional labour. Fourth, there is a split between normative and positive analysis, and finally, there is the general use of mathematical expressions, based on axiomatic and reductionist reasoning.

Do the modern and post-modern periods overlap or are they quite distinctive? Is postmodernism a logical extension to modernity's enlightenment period where the social sciences were heading in a positivist direction? Certainly, postmodernism challenges modern values, such as career, office, individual responsibility, bureaucracy, liberal democracy, tolerance, humanism, neutral procedures, impersonal rules, and rationality. Kilduff (1997) makes the important point that postmodernism is not anti-modernism, in the sense of negating all modern science's achievements. Nor is it about formulating an alternative set of assumptions, as was described in chapter three, but rather making the claim that it is impossible to have any universal underpinnings of knowledge. This means that there are no rigid boundaries so the art/ science debate in marketing disappears. For the postmodernist truth gives way to tentativeness (Rosenau, 1992). It is not a matter of improving or 
perfecting the social sciences but more about exposing the underlying assumptions and undermining social sciences' foundational claims.

Postmodernism questions the values and constructs that dominate modernity, and believe that concepts such as self, society, community, reason, values, and history need to be critiqued without nostalgia or regret or the utopian values underlying modernist thinking.

'In terms of processes, modernism is more interested in continuities, progressions, stable order, and harmony. Postmodernism considers these processes to be illusory and fictional and argues that the micropractices of everyday life, discontinuities, pluralities, chaos, instabilities, constant changes, fluidities, and paradoxes better define the human condition' (Firat \& Venkatesh, 1995 p. 243).

However, postmodernism needs to be put into a historical perspective. Postmodern conditions did not suddenly appear as many of the concepts have existed within modernity over a long period of time. It is part of a wider group of ideas and emphases that have been propounded by late $18^{\text {th }}$ century thinkers up to the present day (Jones, Natter \& Schatzki, 1993). Basically, such thinkers have been conservative with a stress on concreteness, local particularity and diversity. The objective was to seek an understanding of the norms in society by studying their embeddedness in actual history. This can be contrasted with rationalism with its emphasis on progress and change. Notwithstanding the above, romanticism also has been a ghost haunting contemporary accounts of postmodernism. What postmodernism has done is to accentuate these conditions and draw them together under one umbrella. 
If the underlying forces of postmodernism have been present in modernity for some time, what led to the current insurgence of postmodernism? A number of factors can be attributed to the occurrence (Firat, 1995). At a philosophical level there has been the challenge to foundational knowledge and the growing acceptance of relativism, especially as a result of the work of Wittgenstein in 'Philosophical Investigations' where he postulated the ideas of language games and forms of life. This has led to a challenge to moral certitude whereby values have been reduced to contextual situations. According to Schatzki (1993) the way many thinkers have negotiated this turbulent water is to rely on theory and argument. However, postmodernism asks that intellectuals disregard this comfort blanket and instead of seeking cognitive defence just get on acting and judging with what they believe. Therefore, this means that there is a general disregard of theory, that is, general, abstract ideas, which can guide thinking.

The basic theme of modernity is that science triumphs over superstition. However, the role science plays in society has been attacked for a number of reasons (Rosenau, 1992). First, modernism has been accused of not producing the dramatic results, mostly due to inflated expectations and unfulfilled promises. Another related and contributing factor is the abuse and misuse of modern science especially by subjective political policy which has relied on discreet 'scientific facts' to push a certain course of action. A third factor is the gap between modern science working in theory and how it is undertaken in practice. The postmodernists charge that it has not lived up to its formal standards. A further charge is that modern science is unable to remedy the major problems facing the twenty-first century, such as hunger, poverty, pollution and global warming. Also, postmodernists have argued that 
science has ignored the metaphysical dimension of human existence, which leads to the accusation that modern science has nothing to say about ethical issues.

Therefore, the above reasons have contributed to the reaction against the failures of scientific social science to generate knowledge which is a coherent solution to understanding societal problems.

In summary, it is postulated that science and technology has been privileged by modernism at the expense of cultural analysis and symbolic representation. Such a limited view of the consumer as a cognitive agent has been at the expense of other conceptions, for example, the emotive drivers of consumption.

\section{Herding Postmodernism}

As has been noted, postmodernism is a wide and diverse topic so the question arises as to how sense can be made of it so that it addresses the issue regarding the gap between academic research and practitioner activity? In short, can any sense be made out of the topic of postmodernism? Best and Kellner (1991, p. 2) have stated that 'There is no unified postmodern theory, or even a coherent set of positions'. What is needed is a framework whereby certain dimensions are made explicit and themes, based on selected authors, are explored. In developing such a framework it is acknowledged that in a certain sense such an approach could be seen as being antipostmodernism.

'Clearly, communication, knowledge and language are dependent on distinctions and the classificatory schemes or typologies that are their social science counterparts. It is only when distinctions are transformed from heuristic devices into reified ontological realities that they become dualistic. What has come to be defined as the problem of dualism occurs when polarized distinctions are combined with an 'episteme representation' 
wherein what is distinguished as 'this' or 'that' is reified as an ontological reality rather than merely a provisional, subjectively significant, and hence contestable, ordering of 'things'. Dualistic theorizing, then, commits the fallacy of misplaced concreteness since it believes that the distinctions made as part of ordering 'reality' or organizing the world are accurate or true representations or a reality beyond, and as if it were independent of, the theorist.' (Knights, 1997 p. 3)

Goulding (2003) too sees the distinction between the types of postmodernism as being based on a modern approach. 'Nonetheless, although there are clearly two different perspectives on the nature of the postmodern experience, to juxtapose them simply as opposites would be to adopt a modernist strategy of classification into an "either" "or". Conversely, maybe a more realistic focus would be to look for both' (Goulding, 2003 p. 154).

However, distinctions need to be made, especially as the topic is so vast. As noted by Parker (1992), although the questions of post-modernity and postmodernism are interrelated, they need to seen as analytically distinct. 'To collapse them, as some authors are in danger of doing, will lead to confusion on both counts' (Parker, 1992 p. 2). Therefore, the framework needs to be seen as a guide only. It is not definitive, and certainly, the authors overlap in many respects. An analogy can be used to understand the framework. If you think of postmodernism being a large paddock, the question arises as to where in the paddock certain postmodernists tend to graze? By understanding such a framework, it is possible to make sense of postmodernism and select one quadrant to develop ideas which will allow us to answer the central question of the thesis. 


\begin{tabular}{c|c|c|}
\multicolumn{1}{c}{} & \multicolumn{1}{c}{ AFFIRMATIVE } & \multicolumn{1}{c}{ STRONG } \\
\cline { 2 - 3 } $\begin{array}{c}\text { CULTURAL } \\
\text { ANALYSIS }\end{array}$ & Post-modernity & End of history \\
& Jameson & Baudrillard \\
\cline { 2 - 3 } $\begin{array}{c}\text { PHILOSOPHICAL } \\
\text { ANALYSIS }\end{array}$ & Pluralism & Nihilism \\
\cline { 2 - 3 } & Rorty & $\begin{array}{l}\text { Foucault } \\
\text { Derrida }\end{array}$ \\
\hline
\end{tabular}

The first dimension employed by the framework is that made by Rosenau (1992), that is, the difference between affirmative and strong postmodernism, as mentioned earlier. Strong postmodernism ${ }^{14}$ is a 'pessimistic, negative and gloomy' belief regarding the possibilities of there ever being a legitimate social science (Rosenau, 1992 p. 15). The claim is made that all interpretations are valid and that the search for causality is a waste of time. Therefore, what is left is the critique of existing work.

Affirmative postmodernism ${ }^{15}$, on the other hand, retains the possibility of making discriminations between different interpretations. Therefore, a social science is possible that 'embraces and does not exclude the world, reality, history' (Derrida,

\footnotetext{
${ }^{14}$ Termed sceptical postmodernism by Rosenau, and critical postmodernism by Firat

${ }^{15}$ Termed affirmative postmodernism by Rosenau, and celebratory postmodernism by Firat
} 
1988 p. 137). The affirmative postmodernist demands rigorous standards of enquiry when undertaking radical interpretations.

According to O'Shaughnessy et al (2002), the French postmodernists are extreme in their views and can be equated with strong postmodernism. On the other hand, the affirmative postmodernists are less dogmatic and sceptical about rationality and are associated with American thinking.

The other dimension of the framework is that of cultural analysis versus philosophical analysis. Brown (1999) makes a distinction between three types of postmodernism, namely art/architecture, social/economic, and philosophy, as outlined in the previous section 'defining postmodernism'. Schatzki (1993) also makes a distinction between philosophical postmodernity and social history. In this case, social history is taken to mean the same as cultural analysis. Cova and Elliot (2008) argue that a distinction needs to be made between researchers who are interested, on the one hand, in post-modernity, that is , the study of the shift from modernity and understanding how the world is changing, and on the other hand, an interest in the postmodern turn, which involves a specific philosophical perspective, with ontological and epistemological assumptions. Parker (1992) too makes the distinction between post-modernity as a historical periodization and postmodernity as a theoretical perspective, which is epistemological in nature as it addresses the problem of how we know about the world.

Therefore the cultural analysis perspective is concerned with changes in the mode of production within capitalism, where there has been a shift from production to 
consumption and an expansion of felt consumer needs. This has meant that appearances have become important with an emphasis on style, surfaces, and spectacle. A consequence of this shift has been the erosion of the boundaries between high and low cultures (Lutz, 1993). Of special significance for marketing discourse, what both Brown $(1994,1999)$ and Firat et al (1995) have emphasised, is the key concepts of fragmentation, dedifferentiation, hyper-reality, and pastiche.

The second dimension is that of a philosophical perspective. Postmodernism has implications for not only what is researched but how it is researched. In other words, the ontological, epistemological and methodological issues need to be teased out with reference to strong versus weak positions.

It will be noticed that certain authors have been selected to represent each quadrant. This is not to say that they totally represent the quadrant, but more that each author can be used to exemplify the main thrust of the quadrant. For instance, Foucault has a lot to say about the philosophical implications of his postmodern stance, but this does not mean that he also has something to say about cultural analysis. Why adopt this approach? First, it allows us to make sense of the postmodern dilemma, rather than give a superficial overview of postmodernism and second, by comparing and contrasting the selected authors it is anticipated that a greater grasp of the subject will be achieved, thus allowing further development of the thesis.

The choice of authors was justified as follows. Jameson ,Foucault, Derrida, Baudrillard were chosen due to their work being sited by Brown (1993,1994, 1995, 1995a, 1996) and Firat and Venkatesh (1995). Also, Hassard (1994) bases his critique of postmodernism using three leading postmodern writers as examples, 
namely Jean Baudrillard, Jean-Francois Lyotard, and Jacques Derrida, and O’Shaughnessy et al (2002) cites Jacques Derrida, Richard Rorty and Michael Foucault as three influential postmodern writers and mentions that the term 'postmodernism' was coined by Frederic Jameson.

\section{Jameson}

The purpose of this section is to explore Jameson's ideas regarding postmodernism.

The section will begin by stating the arguments why Jameson should be viewed as an affirmative postmodernist, fitting into Quadrant One of the framework. This will be followed by an explanation of Jameson's belief that postmodernism is a completely new epoch. Three important key concepts will then be explained, namely pastiche, fragmentation, and schizophrenia.

Frederic Jameson can be viewed as one of the foremost contemporary Marxist cultural critics (Best, 1991). Over the last 30 years he has developed a neo-Marxist theoretical perspective ${ }^{16}$ and has been involved in many theoretical debates in the area of cultural and literary studies, including such diverse topics as the novel, the video, fairytales and postmodernism.

\footnotetext{
${ }^{16}$ Many Marxists have adopted a postmodern stance due to the authoritarian and dogmatic traditional Marxist approach. Two groups who are attracted to postmodernism are post-Marxists and neoMarxists but they have different views on Marx's concepts and they disagree regarding the appropriateness of applying certain Marxist concepts to postmodernism. The post-Marxists give up the original axioms of Marx due to the changed contemporary scene whereas the neo-Marxists retain the core terminology but change concepts at the margin, for example, Jameson's concept of time and space (see below).
} 
Why is Jameson an affirmative postmodernist? Firstly, he is sympathetic to the author ${ }^{17}$ and also to the importance of agency that is critical to his neo-marxist beliefs (Rosenau, 1992). He sees the possibility for new forms of networks in a postmodern society which would allow political practice to build a new socialist society. This stance makes him open to the criticism of adopting a meta-narrative, which is complete anathema for most postmodernists. Also, the role of rationality still plays a part. 'This insistence on the need for a vision of the whole is tied to an essential faith in the powers of critical reason' (McGowan, 1991 p. 148).

Jameson introduces the plea for a meta-narrative by utilizing the concept of cognitive mapping. Shields (1992) has noted that the tenor of Jameson's 1984 article, 'Postmodernism, or the Cultural Logic of Late Capitalism', shifts from the fragmentation of American culture to the spatial terminology of cognitive maps. 'The political form of postmodernism, if there ever is any, will have as its vocation the invention and projection of a global cognitive mapping, on a social as well as a spatial scale' (Jameson, 1984 p. 92).

Shield's argues that the notion of cognitive mapping is synonymous with functionalist concepts, especially those introduced by Parsons, whereby a systematic social structure is posited. This can be seen when Jameson suggests that for

\footnotetext{
${ }^{17}$ With postmodernism the role of the author is diminished, whereas the reader and the text itself gain importance (Rosenau, 1992). Postmodernists see the reader as not being passive but involved in the interpretation of the text, taking responsibility for what they read. As it is not a question of gaining 'truth' or 'knowledge', the reader is seen as reading for pleasure so as to gain new insights.

In the conventional, modern sense the author is the arbiter of meaning. The role of the author in this case is that of legislator, in the sense that the author has expertise which s/he has privileged access to the truth, reason, and scientific knowledge. It is a mistake to give the modern author the final word as we do not know about the relationship between the author and the text. The postmodernists see authorship as being, based on a platform of power, because any document that is written has some responsibility assigned to it so therefore it has a legal connotation whereby blame can be apportioned.
} 
individuals to overcome alienation in a fragmented, postmodern world the solution 'involves the practical reconquest of a sense of place, and the construction or reconstruction of an articulated ensemble which can be retained in memory and which the individual subject can map and remap along the moments of mobile, alternative trajectories' (Jameson, 1984 p. 90). The idea is that individuals can develop their own cognitive map of what they deem to be important entities so that the fragmented world makes sense.

However, it needs to be asked as to the type of map Jameson is alluding to if there are no fixed points? If his view of postmodernism is accepted, then different directions obtained from a map are dependent on the viewpoint of the map reader. This renders such a map, with different viewpoints, unreadable. Unfortunately by invoking such a map Jameson is 'giving rise to the ironic, functionalistic overtones of his description of the postmodern political project' (Shields, 1992 p. 53). It is for this reason that I argue that Jameson is an affirmative postmodernist. For Shields, an affirmative postmodernist is one who presents tools to discuss contemporary social issues (Shields, 1992 p. 50). Whilst Rosenau (1992, p. 69) implies that Jameson is a skeptic, she places Jameson firmly in the affirmative camp due to his sympathetic stance to the author and the importance of agency with respect to his political agenda. Brown (1995 p. 99) sees him as an affirmative postmodernist, whereas Firat (1995 p. 244) places Jameson as a skeptic in the sense that he advocates a radical break from modernism, but he does admit that Jameson wants a return to moral utopianism. It is Jameson's implied belief of 'hope' that makes him a candidate for an affirmative postmodernist. 
According to Grahams and Doherty (1992), Jameson uses the term 'postmodernism' as a consumer stage of capitalism, rather than the more widely accepted notion of production being the basis of modernism. For Jameson consumerism is a mode of production, where cultural production performs special functions and takes a specific form. It is important to note that he is not implying that postmodernism is homogeneous or that it is another stage in the evolutionary stage of society. Postmodernism is not another extension, or variation of a theme, of modernism but is a distinct phase on its own. 'It is essential to grasp 'postmodernism' as not a style, but rather as a cultural dominant: a conception which allows for the presence and coexistence of a range of very different, yet subordinate features' (Jameson, 1984 p. $56)$.

Jameson claims that there is a sharp discontinuity with modernism and that the two epochs are quite separate and different. "All the straws in the wind seem to confirm the widespread feeling that 'modern times are now over' and that some fundamental divide, some basic coupure, or qualitative leap, now separates us decisively from what used to be the new world of the early or mid-twentieth century, of triumphant modernism and the revolt against positivism and Victorian or Third Republic bourgeois culture' (Jameson, 1988 p. 17).

Therefore, for Jameson, postmodernism as an entity is not part of a development, nor can it be seen as a form of progress in the modernist sense. Consequently the underlying assumption presented by postmodernism is that there is no linear history. All that can be celebrated is the here and now. Emancipation, either in a liberal sense or a Marxist position is unobtainable, which for some critics is untenable. 'The 
abandonment of any conception of an ideal form of society can thus be represented as politically disenabling, an implication which worries many, particularly on the political left' (Graham et al, 1992 p. 17).

Jameson argues that since the 1960's there has been a new era whereby the production of culture is integrated into commodity production (Harvey, 1995). This is evidenced by the product life cycle becoming reduced over time. In other words, the cycle rate has increased. This can be seen in diverse items from running shoes to motor cars. The production of culture "has become integrated into commodity production generally: the frantic urgency of producing fresh waves of ever more novel seeming goods (from clothes to airplanes), at ever greater rates of turnover, now assigns an increasingly essential structural function to aesthetic innovation and experimentation' (Jameson, 1984 p. 64). ${ }^{18}$ This has led to a change in consumer habits and a new role for aesthetic definitions.

Jameson believes that culture has been commodified, and consequently has become what could be described as 'shallow'. According to Harvey (1995), Jameson makes an emphatic claim about the 'depthlessness' of cultural production. There is a concern for appearances, surfaces and instant impact but with no sustaining power over time. In other words, postmodernism appears to be shallow, or in the words of Andy Warhol, 'deeply superficial'.

As has been noted, Jameson believes that fashion, life cycles, and in turn beliefs, have rapidly increased but without any real transformation taking place. Whilst these

${ }^{18}$ See A.G. Ramos (1981) The New Science of Organization, Chapter 5. 
changes are taking place, it is at a global level rather than a national level. ${ }^{19}$ Such changes have allowed flexible manufacturing and the easy flow of capital resources. Jameson sees that such rapid change at a superficial level, on the surface, leads to the opposite, that is, statis.

Following on from this theme, art has been commodified by the market (Harvey, 1995). Jameson notes how big corporations have become major patrons of the arts, mainly due to the scope that such patronage allows re sponsorship, media coverage, and client entertainment. For example, Meridian Energy sponsors the New Zealand Ballet. Also, another factor has been the buying of up and coming artists' works with the intention of acquiring capital gain.

The role of museums is also criticised. The heritage industry has commercialised history with nothing more than a shallow screen. Their role has been blurred by being, on the one hand, a place for education, and on the other hand, a place for entertainment, for example the Museum of New Zealand Te Papa Tongarewa. Many museums are now competing with theme parks, and in some cases contain theme parks. Consequently there is a shallow link between the present and the past by the use of popular images and simulacra with that history. Jameson's thesis is that postmodernism is nothing more than the cultural logic of late capitalism (Jameson, 1984).

A central term coined by Jameson is that of 'pastiche'. The term refers to a juxtaposition of unrelated ideas, consumer experiences, and historical moments. The

19 Klein re the globalisation of markets 
use of past styles and symbols are combined in a collage so that symbolic codes are mixed. This can be easily identified in fashion and architecture. For instance, a recent fashion style is a frock, a symbol of the ' 50 s, being worn over a pair of jeans, a symbol of baby boomers.

'Indeed, it is arguable that, despite the undeniable importance of dedifferentiation, hyperreality and the others, pastiche is the defining feature of postmodernism. Call it what you will - irony, parody, imitation, medley, quotation, self-referentiality, double coding, in-jokes, the knowing wink, tongue planted permanently in cheek, a refusal to take things seriously, not even taking things seriously - but all of these are characteristic of the pasticheur and nowhere is the pasticheur more prevalent than in marketing' (Brown, 1995 p. 119).

Another important term for Jameson is that of fragmentation. Postmodernists believe that society is disintegrating with respect to the old order. Such trends can be identified by the disestablishment of hierarchies, for example the distinction between high and low culture, and the implied elitism with the distinction between training and education. This in turn leads to a blurring of entities such as philosophy and literature, and science and religion, consequently such dichotomies are rejected. As Goulding (2003) notes, fragmentation in the marketing domain takes many forms. There is the fragmentation of markets into smaller segments, accompanied by the increase of the number of products to serve those segments, as well as the fragmentation of media, as witnessed by the increase of television channels, and the growth of the internet and associated digital media.

One central theme is the notion of 'death of the subject'. For Jameson, there is no place where critical discussion can occur. Pastiche has led to motifs, styles, and themes being recycled with the consequence that there now exists a 'society of the 
spectacle'. This in turn leads to schizophrenia, a term used by Jameson as a metaphor to explain the delusion shrouding contemporary culture. Jameson uses the term to describe a linguistic disorder caused by a break in the signifying chain due to the subject being confronted by a parade of changing images. This fits in with postmodernism with an emphasis on the signifier rather than the signified, and with surface appearances rather than substance. Fragmentation is linked to schizophrenia with the outcome that it stops coherent thinking. This can be linked to increased product cycle rates where the future becomes disconnected from the past, therefore making it hard to maintain any sense of continuity.

The purpose of this section has been to explore the work of Frederick Jameson as an example of an affirmative postmodernist who is interested in cultural analysis. Jameson claims that postmodernism is a separate epoch from modernism, where individuals make sense of their alienation through the process of cognitive mapping. He also utilises three core concepts to what he calls the logic of late capitalism, namely pastiche, fragmentation, and schizophrenia. Whilst Jameson is interested in building a new society, he recognises that society is far from utopia. McGowan (1991) sees his description of postmodernism to be that of despair.

In contrast to Jameson description of postmodernism is that of Baudrillard, who can be viewed as a strong postmodernist, with an interest in cultural studies. The next section will explore Baudrillard's views, which will then be contrasted with Jameson's view. 


\section{Baudrillard}

In contrast to Jameson, the work of Baudrillard will now be explored. This section will begin with a justification as to why Baudrillard has been chosen as a strong postmodernist, and will be followed by an account of four central themes found in his work, namely, communication and the role of media; the concept of simulacra; the notion of representation; and the place of history in the postmodern world.

Baudrillard is viewed as the high priest of postmodernism (Best, 1991; Brown, 1995, O'Shaughnessy, 2002) He was chosen as the representative author from Quadrant Two, that is, someone whose work can be viewed as being concerned with cultural analysis from a strong postmodern perspective, for two reasons. First, he is viewed as the most advanced theorist of society and the media in the postmodern period to date (Best, 1991). That is not to say that Baudrillard ignores any philosophical implications. However, his main thrust has been a critique of society, concentrating on the effect of modern communications.

Second, he is a strong postmodernist as the take-out from his analysis is that of pessimism and gloom. Instead of offering a solution to counter the impact of communications in society, he suggests that the public does not respond. 'The only way of subverting the universe of the media, the simulated world of hyper-real media objects, is through the fatal strategy of silence or passivity. Rather than complaining about the alienating impact of the media, the 'way out' is simply not to respond at all. This solution is an utterly pessimistic one. It compounds rather than reduces the problem of alienation and reification' (Schweitzer, 1991 p. 38). 
Baudrillard begins with a neo-Marxist framework to explore the notion that production has been replaced by consumption as the guiding principle of social theory. However, he came to the conclusion that Marxist theory was unable to conceptualise the importance of language, signs, and communication in general. $\mathrm{He}$ criticises Marxist theorists for trying to search for meaning below the surface. In doing so he positions himself as being more concerned with the surface, focusing on the here and now.

In his exploration of the impact of communication on society, Baudrillard comes to the conclusion that images have become commodities. He believes that Marxism is outdated with its central theme of commodity production because capitalism is now concerned with the production of signs, images, and sign systems, so much so he proclaimed that the USA is a society of the spectacle. Harvey, (1992) notes that in 'L'Amerique' Braudrillard refers to the USA as a giant screen. 'Baudrillard carries the works of Kafka, Orwell, and McLuhan to their logical conclusions. If 'the social' ever existed as an object of representation, study, or collective action, it no longer does, or else it has been transformed into an object for manipulation' (Hollinger, 1994 p. 128).

He argues that as the output of production has been replaced by models, spectacles, and simulation, this in turn has meant that people have less of a relationship to an external reality. Taking an extreme position, Baudrillard believes that image has replaced direct experience. Central to this notion of image is the role of communication. It is communication that has taken over from production. The implication of this is that there is more information but less meaning. For example, 
is economic performance measured by GDP or a strong exchange rate? What is the relationship between such variables? The result of this deluge of communication sounds bites is that there is a collapse of modern culture giving rise to postmodernism.

'In Baudrillard writings, the masses, the silent majorities, passively consume commodities, television, sports, politics, mass-produced simulations to such an extent that traditional politics and class struggle become obsolete. This is the era of consumer culture, and consumer culture for Baudrillard, is effectively a postmodern culture: traditional distinctions and hierarchies have collapsed, polyculturalism is acknowledged; kitsch, the popular and difference are celebrated' (Sarup, 1993 p. 166).

Baudrillard has been a central figure in the critique of the media. For Baudrillard (1978), television has become dominant in households. The consequence of this is that signs, images and simulations become reality, or in some cases, 'more real than real'. Advertising invades public space, which in turn means that there is a lack of private space. This can be witnessed in the Liverpool down town shopping mall where there is a giant screen playing 'Eastenders' to shoppers on a Sunday afternoon. ${ }^{20}$ Intimate details of lives are exploited by the media, whether they be

\footnotetext{
20 'The mall offers the previously unexperienced luxury of strolling between stores which freely offer their temptations without so much interference or glare from a display window. The central mall, a combination of rue de la Paix and the Champs-Elysees is adorned by fountains and artificial trees. Kiosks and benches are completely indifferent to seasonal changes and bad weather...... Here we are at the heart of consumption as the total organisation of everyday life as a complete homogenisation..... Work, leisure routine and culture, all previously dispersed, separate and more or less irreducible activities that produced anxiety and complexity in our real life....have finally become mixed, massaged, climate controlled and domesticated into the simple activity of perpetual shopping. All these activities have finally become desexed into a single hermaphrodite ambiance of style.' (Baudrillard 1988 p34)
} 
celebrities or people taking part in 'reality TV' programmes, for example, Big

Brother. $^{21}$

More and more information is presented but with less meaning. As a coping mechanism to overcome information overload, viewers take more note of the signifiers and ignore the signified. This can be seen in the recent battle for the seven pm news slot where the signifiers, that is, Susan Woods, Paul Holmes and John Campbell battle for ratings. The content, that is the signified, pales in importance. All meaning and interpretation disappears with the consequence that the public are unable to critique rationally.

Baudrillard takes the distinction made by McCluhan (1994) regarding hot and cool events. He believes that the media take hot events, for example, sport and turn them into cool events. For Baudrillard, going to an actual game at the stadium he writes, 'Do not believe that it is a matter of the same game: one is hot, the other cool - one is a contest where affect, challenge, mise en scene, and spectacle are present, whereas the other is tactile, modulated, modulated (visions in flash-back, replays, close-ups or overhead views, various angles, etc.): a televised sports event is above all a televised event, just as the Holocaust or the Vietnam war are televised events which one can hardly make distinctions' (Baudrillard, 1979 p. 220).

The consequence of the media turning a hot event into a cool event is that meaning is neutralised and the audience is involved in a one-dimensional experience involving

\footnotetext{
${ }^{21}$ Big Brother is a reality television show, which originated in the Netherlands in 1999. The name is derived from the novel by George Orwell. The show involves a group of people who live in a house and have to face certain challenges. Over time members of the group are evicted, so eventually there is a winner. The show has been produced over five regions, namely UK, Africa, Balkans, Arab States, Pacific and Scandanavia.
} 
passive absorption rather than an active processing of meaning. For Baudrillard, all television programmes are 'cool' events.

Therefore, the media pander to the masses, reproducing their taste, fantasies and way of life so that the public would rather have spectacle, entertainment and escape instead of producing meaning for them.

Baudrillard writes about the 'precession of simulacra', that is, changing relations between, on the one hand, signifying what is 'real' and representing the 'imaginary'. This notion of the simulacra can be used to understand the contemporary cultural situation. Baudrillard believes that there are four stages. The first is the metaphor of the mirror. There is a reflection from the real empirical world to our senses. This view of reality is the foundation of scientific methods such as verificationism and falsificationism.

The second stage, developed in the nineteenth century, is the metaphor of the mask where it is postulated that it impossible to gain a good reflection as our senses are being blocked therefore they need unmasking. This led to the development of structuralism and can be seen in the work of Marx and Freud, where insights are found beneath the empirical world. This type of thinking is the basis for Critical Theory in the twentieth century (Jones et al, 1993).

The third stage is that of simulacra. Developed in the late twentieth century, there has been the substitution of signs or representations to stand for an object, for 
example, logos that sum up brand associations. ${ }^{22}$ A simulacrum could be a copy of something that has been lost or maybe it did not exist in the first place. It blurs distinctions between real and imaginary, signifier and signified, so consequently there is no true or false.

The fourth and final stage is where everything is pure simulacra, with no relation to reality. However, Baudrillard believes that the fourth stage has not yet been reached and that culture is still locked into the third stage. One important concept in this stage is that of hyper-reality. This is where there are no boundaries, fact and fiction are fused and the theory practice distinction disappears. In such a state, nostalgia becomes an important force, which can be seen in the rise of retro-marketing. Also, links to the past, where artefacts are made to look authentic are prominent, for example, the Guinness factory tour in Dublin. Other examples of hyper-reality are theme parks and computer games, where reality takes over. Baudrillard cites Disneyland as an example of hyper-reality. He argues that by acknowledging its artificiality it makes it more authentic.

Following on from the above critique of media, Baudrillard uses the term 'implosion' to describe the effect of simulacra and media on society. Whereas the modern world, that is, the western industrial world, was marked by the expansion of national boundaries, products, science and technology, termed 'explosion', the postmodern world is implosive in the sense that they fall in on themselves due to the images and information that saturate society. Separate spheres disappear and divisions between

\footnotetext{
${ }^{22}$ Re the importance of brand image being nothing more than a sum of its associations, both positive and negative. The logo is a reflection of brand image.
} 
social classes and political ideologies disintegrate. In this sense society implodes into a continuous, undifferentiated barrage of simulacra.

Representation is another important concept, which can take many forms (Rosenau, 1992). It can be in the form of delegation, for instance, a representative in parliament. It can be in the form of a resemblance, for example, a portrait of the Queen or landscape. Following on from this a representation can take the form of a replication as in a photograph, as well as repetition, such as a writer putting their thoughts on paper. Substitution is another form of representation, for instance, a lawyer representing a client, and finally, duplication, where a photocopy represents the original.

A representation, for example, Renee Magritte's 'ceci n'est pas une pipe', refers to some other item, which in turn is a representation of something else, so ultimately all representations refer to other representations, so nothing is authentic with the consequence that the true and false distinction fades. As it can be seen, the concept of 'representation' implies that there is something out there which is valid with its own meaning based on some notion of truth.

Language has only referential status with the use of words, images, meanings and symbols. Therefore there is no direct relationship to the real world. Any reference is only symbolic. Following on from this, all representations are mediated by language so therefore are not directly reality related. 
Representation assumes an ability to reproduce and duplicate an external reality and in doing so implies that things exist in the real world which consists of knowledge, universality, and containing truth.

Finally, history and time holds a special place in Baudrillards theory of society. He rejects history for its own sake and relegates its role to the side-line in the scheme of human affairs. In particular he rejects the idea of causal progress (Baudrillard, 1983). He also rejects the idea that concepts should have logocentric status, that is, man's status is privileged at the expense of other beings or subjects (man as the centre of the universe). Humanism is also rejected as it is seen as being a logocentric metanarrative based on an internally fixed frame of reference.

Following on from the above, linear time is rejected. 'Linear time is viewed as offensively technical, rational, scientific, and hierarchical' (Rosenau, 1992 p. 68). It removes joy from life because it is tied up with the notion of work, that is, modernist notions of production. Time is a human creation so is therefore arbitrary, scientific and rational. For Baudrillard, time can be shaped, unlike the modern idea that sees time as linear or cyclical. In other words, it has a certain shape, but for Baudrillard time can be compressed, twisted, distorted, or even discarded. The end cannot take place because everything has already happened so history has run out of steam and nothing new can occur. ${ }^{23}$ Consequently retrospection is the only alternative, which gives rise to the importance of nostalgia.

\footnotetext{
23 'We are in the process of wiping out the entire twentieth century, effacing all the signs of the Cold War one by one, perhaps even all trace of the Second World War and all of the political or ideological revolutions of the twentieth century ..... At the rate we are going we shall soon be back at the Holy Roman Empire. And perhaps this is the illumination this fin de siecle offers and the true meaning of that controversial formula "the end of history". The fact is that, in a sort of enthusiastic work of mourning, we are in the process of retracting all the significant events of this century, or whitewashing
} 
According to Brown (1995) there are two responses to Baudrillard's position. One can either push the limits of the system by increasing consumption, or become completely apathetic and treat life as a joke. As will be discussed in the next section, there is no distinction between what is real and what is an illusion. Theories of modernity no longer apply to images, media or consumerism, so things will selfdestruct. With ironic cynicism, Baudrillard hopes that the process of decay will speed up with the consequence that the system destroys itself.

In summary, this section has shown that Baudrillard is a strong postmodernist with a sceptical view of culture. He believes that the output of production is images, with increasing sound bites but with less meaning. Through the process of simulacra, reality is merged with models, spectacles and simulations. By exploring the concept of representation, Baudrillard claims that all representations refer to other representations, consequently nothing is authentic. Finally, he rejects the notion of linear progressive history as he views this as a modernist concept, linked with the notion of production. From a cultural perspective, Baudrillard see no future, with the system ultimately self-destructing.

it, as if everything that had taken place (revolutions, the division of the world, exterminations, the violent transnationality of states, nuclear cliffhanging) - in short, history in its modern phase - were merely a hopeless imbroglio, and everyone had set about undoing that history with the same enthusiasm that had gone into making it. Restoration, regression, rehabilitation, revival of the old frontiers, of the old differences, of particularities, of religions - and even resipiscence in the sphere of morals. It seems that all the signs of liberation achieved over a century are fading and will in the end perhaps be snuffed out one by one: we are engaged in a gigantic process of revisionism - not an ideological revisionism but a revisionism of history itself, and we seem to be in a hurry to finish it before the end of the century, secretly hoping perhaps to be able to start again from scratch in the new millennium.' (Braudrillard, 1994 pp 32-33) 


\section{Derrida}

The last two sections have explored two postmodern authors who have written extensively about postmodernism from a cultural analysis perspective. It has been argued that Jameson is an affirmative postmodernist as he does possess some hope for the emancipation of society. Baudrillard on the other hand has some interesting comments to make about the influence of modern communications on the culture of the western world, but ultimately he can be viewed as a strong postmodernist as his 'end of history' point of view offers no hope for emancipation. Rather, he postulates a nihilist, 'roll over and surrender' approach.

This now takes the chapter to another important dimension of the framework, that is, postmodernism from a philosophical perspective. Two authors, Derrida and Foucault have been chosen to represent the Nihilism quadrant primarily because of their influence on the theory of social science (Brown, 1993,1994, 1995, 1995a, 1996; Firat and Venkatesh, 1995; and Hassard, 1994), and also because they have different points of view.

Derrida is a strong postmodernist because he does not provide any direction for future research. He provides no way forward from the ying and yang of deconstructionism. Unpacking dichotomies so as to find and locate different meanings may give some insights, but at the end of the day further consideration needs to be given to knowledge production.

In exploring the work of Derrida four themes, central to his philosophical position, have been selected. The first theme is the role of language, which leads to the 
second theme, that of difference and the concept of play. The third theme is the notion of deconstruction, with the final theme being the role of the author and the importance of text.

Language plays an important part in Derrida's analysis. For Derrida, language does not simply reflect reality but helps to constitute it. Language shapes our experiences and our judgements. Language works by means of transference from one kind of reality to another and is thus essentially metaphorical. Metaphor allows proliferation of meaning. Metaphors are one of the ways in which many kinds of discourse are structured and consequently powerfully influence how we conceive things, for example, 'time is money'.

This means that language has a rhetorical status, therefore literature should not be seen as a poor relation to philosophy. Derrida believes that literature should be on an equal footing with philosophy (McGowan, 1991).

Derrida was concerned about the instability of language (Brown, 1995). A distinction can be made between the signifier, that is, a word, sound or image, and the signified, which is the referent of the sound or the mental object that is evoked. For Derrida, this relationship is completely arbitrary and based on convention. Therefore, this relationship is by no means stable, consequently meaning becomes difficult to determine. This leads to the concept of differance.

This key concept for Derrida can be described as a form of self-reference where terms contain their own opposites thereby making it difficult to comprehend singular 
meanings. By spelling the word difference with an $a$, Derrida combines the two senses of the French word differer, that is, to differ with regard to space and to differ with regard to time. The idea behind this is that there is never a fully present reality. Rather, the world is continually deferred both in time and space. This affects both the signified and the signifier. 'The signified concept is never present in and of itself, in a sufficient presence that would refer only to itself. Essentially and lawfully, every concept inscribed in a chain or in a system within which it refers to the other, to other concepts, by means of the systematic play of differences' (Derrida, $1982 \mathrm{p}$. 11). Therefore, differance always runs ahead of us, consequently it cannot be located in any specific place and is not something that can be grasped in the present.

'The argument that Derrida advances can be said to be essentially against the idea of a fully present reality which we normally consider the world to be, directly and unitarily available to our understanding, and what is posited instead is a world that is continually deferred, postponed in space and time' (Cooper, 1986 p. 312).

McGowan (1991) characterizes Derrida's position as negative mysticism. He places difference outside philosophy and outside language, but that does not mean that we can get outside the system. According to Derrida our knowledge of differance comes from within. It makes all thought possible, that is, it puts thought into play. Play involves the liberation of the signifier from its dependence on the signified. Therefore, there are an infinite number of possibilities within the finite system of language.

The use of the word play does not mean playful in the sense of games, but play in the sense of movement in a machine (McGowan, 1991). For instance, play in the axle of a bike allows the wheel to rotate. Not enough play and the wheel seizes; too much 
play means the wheel is unstable. Play in this sense allows for movement, and if too much play, instability.

Derrida also introduces the term 'sous rature'. This is where the word is printed and then it is crossed-out. What this is conveying is that there is not a one to one correspondence between the signifier (the image) and the signified (the concept). If a sign is read its meaning is not immediately clear to us, which means that language is not stable. Brown suggest that the term 'marketing' should be treated accordingly, Imarketing, as its meaning is unclear.

This leads to the next theme, namely the concept of deconstruction, which is both post-phenomenological and post-structural. The approach of deconstruction is to read a text and show how it has failed due to inconsistencies and paradoxes contained within the text.

People have been given to think in binary terms, such as good-evil, man-woman, mind-matter, true-false and in doing so a hierarchical order is set up implying one is better than the other. Once an oppositional pair has been set up a choice needs to be made. Derrida places them in tensional play with one another so that there is an endless play of thought and language around them.

Every text can be deconstructed, that is, shown to move toward play. But what does this mean? McGowan (1991) states that Derrida must resist simply preferring one binary opposite at the expense of the other, that is, reversing hierarchies, for example, by adopting a feminist stance as opposed to a masculine one. 
Derrida wants an endless oscillation between terms. This ultimately leads to an impasse between thesis and anti-thesis. In fact, Derrida's own thought swings between the construction of identities and deconstruction, for example, when play reigns supreme.

Is the reading of a text the final court of appeal? Derrida's famous remark "There is nothing outside the text" is saying that all human life is textual in a broad sense and that a sign is not a referent to some immediate being or object. 'For Derrida, there are no lost origins, no natural guideposts, no determinate meanings - only the infinite play of signifiers in texts' (Hollinger, 1994 p. 96).

The role of the author is diminished, whereas the reader and the text itself gain importance (Rosenau, 1992). Derrida sees the reader as not being passive but involved in the interpretation of the text, taking responsibility for what they read. As it is not a question of gaining 'truth' or 'knowledge', the reader is seen as reading for pleasure so as to gain new insights.

As Rosenau (1992) notes, in the conventional, modern sense the author is the arbiter of meaning. The role of the author in this case is that of legislator, in the sense that the author has expertise which s/he has privileged access to the truth, reason, and scientific knowledge. It is a mistake to give the modern author the final word as we do not know about the relationship between the author and the text. Derrida sees authorship as a bourgeois phenomenon, based on a platform of power, because any 
document that is written has some responsibility assigned to it so therefore it has a

legal connotation whereby blame can be apportioned.

It is interesting to note that authorship in time may disappear as is already apparent by the use of ghost writers for books, speech writers for politicians, screenwriters for television and movie scripts, and copy writers for advertisements. The role of such writers has meant that there is no responsibility at an individual level.

Derrida wants to reduce the author's authority. He denies that the author wholly accounts for a texts meaning. The reader is seen as an interpreter, rather than a passive, absorber of an authoritative viewpoint. The reader as interpreter sketches out various options thereby making knowledge relative to a community. However, it needs to be noted that for Derrida, there is not one interpretation that is better than another.

With regard to texts, no two are alike and with a second reading the text is never identical. A postmodern text generates interpretations as it is not a passive text with intended meanings but more 'readerly' text.

'The text has become the object and the task becomes the disentangling, or deconstruction, of the free play of differance in the discourses that inhabit the writing. No one discourse is given privilege over another and analysis becomes celebration as the old assumptions of categorical priorities fall away' (Graham \& Doherty, 1992 p. 5).

Derrida uses the notion of intertextuality where every text is related to every other text, so consequently they impact upon each other. The meaning of a text resides not in the production of a text but in the reception of the text. 
If everything is a text then the world becomes global and inclusive thereby rendering everything to be of little value. This means that there are an infinite number of interpretations of any scenario and each interpretation is valid (Rosenau, 1992). This means that it is possible to have both 'official' and 'non-official' realities.

Hollinger (1994) notes that if text is interrelated with all other texts it means that there is nothing beyond language to confer meaning on the text, consequently the locus of meaning becomes culture itself as encoded in language. However, the problem is that if one can only interpret from the text then how are intentions accounted for in describing individual action? Another problem, posed by Hollinger (1994), is the determination of confirmation procedures for testing an interpretation.

It has been argued that Derrida ethically condemns the monolithic version of western philosophy because it suppresses the difference of 'others', that is, the weak, the poor, the marginalised. His strategy to disrupt this monolith so as to radically challenge its imposition by introducing the concept of differance.

Unfortunately, Derrida's approach is basically pessimistic. 'Derrida offers few concrete reasons for hope' (McGowan, 1991 p. 110). He is unable to say how the notion of differance is translatable into a programme of political action. To work within the system using deconstruction is only to highlight a play that was always there, albeit hidden most of the time. If one was to move outside the system one would need to embrace mysticism, as language is synonymous with thought.

Critics of Derrida argue that one needs to know an author's intention (O'Shaughnessy, (2002). If the author is not included in the relevant interpretation 
how does one know the purpose of the author? This can be seen especially in the area of market research, where often interpretation is required to understand the researcher's (author) intention regarding the question.

Also, as (O'Shaughnessy, (2002) queries, the text and author are mutually interdependent as many interpretations are based on beliefs about the author. But for Derrida it is about the meaning of a sign as a signifier, thereby focusing on symbolic meaning rather than referential meaning. Therefore there is a shift from what is said to how it is said. However, one questions how easy it is to separate referential meaning from symbolic meaning.

There are further arguments against deconstruction: First, it would seem that texts only refer to other texts, which in turn leads to a web of inter-related texts, commonly called intertextuality. Therefore there is no final text, and by such a reference system it leads to an infinite regress. It is not about there being 'no truth' but rather Derrida questions the nature of truth. Following on from this, it would seem that meaning becomes undecidable. Second, there appears to be only interpretation but this is done within a language so one is trapped within the concepts and traditions of western metaphysics. Derrida offers no way out of this dilemma.

Stern (1989) argues that because deconstruction leads to meaninglessness, it has received a lot of criticism, but she notes that deconstruction may be useful in the area of consumer research by questioning the assumptions behind normally useful terms. 'Deconstruction is perhaps best viewed as the most recent source of potential insights 
form literary criticism to inspire or provoke consumer researchers studying meaning, values, and pleasures in the marketplace' Stern, 1989 p. 326).

The task of this section was to outline the work of Derrida as a strong postmodernist, with the aim of deciding whether such an approach could be used as the basis for a new research approach for marketing management based on the linguistic turn in philosophy. The conclusion is that, although this central theme of deconstruction has merit in certain marketing spheres, for example, consumer behaviour (as noted above), it does not provide a basis for a research programme for the marketing management school. As Goulding (2003) notes, sceptical postmodernists view the task of research as a futile exercise.

\section{Foucault}

To contrast the ideas of Derrida, it is apposite to explore briefly the work of Foucault and to determine whether he can provide a basis for a new research programme for marketing management.

Is Foucault a strong postmodernist? Rosenau (1992), Brown (1995), Firat (1995) and Kilduff (1997) place Foucault as a strong postmodernist or the equivalent. ${ }^{24}$ Also, O’Shaughnessy (2002) sees Foucault as a strong postmodernist, coming from the French tradition. Whilst Schatzki (1993) claims that Foucault is neither an anarchist, as he maintains that no system is better than any other system, nor a

\footnotetext{
${ }^{24}$ Firat (1995) uses the term 'critical postmodernism'.
} 
nihilist, ${ }^{25}$ as he believes in individual or group struggles at the local level, based on liberty, autonomy and growth without any form of subjection to others, he does not offer any way to evaluate one struggle against another. Therefore it is posited that Foucault is a strong postmodernist.

Foucault is a historian who breaks the past from the present (Sarup, 1993). Because the past is seen as foreign, the present is relativised. He uses a Nietzschean tactic of critique to look for difference. His method is to start with the present and go back in time until difference is found. Then the task is to proceed forward, tracing the transformation of the situation, noting the connections as well as the discontinuities.

According to Knights (1992), Foucault's extensive writing can be broken down into three distinct periods, namely that of the archaeological period, where he concentrates on the role of language and determining a set of rules and procedures for making statements true; the genealogical period where he explores the concept of power, especially in relation to knowledge?]; and the ethical period where he was particularly interested in the ways individuals subjectify themselves through discourse and practices. His focus of study during this period was on the domain of sexuality. In terms of the thesis, it is the first two periods which will be explored in this section, as they have the potential to impact on the marketing discipline.

Language has an important part to play in Foucault's analysis. Structuralism sees all relations as linguistic, symbolic, and discursive whereas Foucault shifts the emphasis from linguistic determination to a view that individuals are limited by power.

\footnotetext{
${ }^{25}$ The term nihilism means the denial of the possibility of any affirmations and a rejection of the very prospect of knowledge altogether.
} 
All spheres of life are subject to investigation, regulation and surveillance by means of discourse. Discourses are 'language games' in which the power is circulated. There is no pure subjectivity outside these systems of thought. Discourses can be compared to Kuhn's paradigms in so much that they structure the field of knowledge.

In other words discourse can be seen as modes of knowing, comprising a set of linguistic rules which allow some types of thought but not others. Therefore, discourses determine what counts as knowledge in any given situation, whether it be to determine madness, sickness, or truth. Discourse shapes our desires, thoughts, and our identity. Following on from this, it can be seen that the individual is a construct of language.

As Sarup (1993) notes, Foucault is against ideology as he thinks that ideology stands in opposition to something, and by taking such a position it is positing a truth statement. But for Foucault, truth statements are produced in discourses, which are neither true nor false. Also truth statements presuppose a human subject based on classical philosophy, which Foucault opposes. Truth is relative for Foucault so it is impossible to make claims about different historical periods or state claims about the status of different theories. So what position is Foucault writing from? What status is to be ascribed to Foucault's writing?

In the second period Foucault was interested in the strategies and tactics used in exercising power over people. He undertook this study from a historical perspective (Knights, 1992). In studying the institutional practices of places of internment, for 
example, hospitals and prisons, he found the institutions had embedded within them methods for separating the sick from the healthy, the mad from the insane, and the criminals from the law-abiding people. 'In short, these disciplines and institutions exercise power through the 'normalizing' procedures of exclusion and surveillance whereby 'deviants' (e.g. the sick, paupers, criminals) are physically segregated and the 'incompetent' are marginalised through hierarchical distinction' (Knights, 1992 p. 517).

He argues that knowledge gives power over others, and this power therefore allows one to define others. Consequently, knowledge is not about liberation but a mode of surveillance and regulation. It is necessary to somehow transgress the limits of these power relations.

'Following Nietzsche, Foucault argues that knowledge and power are always intertwined in specific ways. Various systems of discourse, and disciplines such as psychiatry, medicine, psychoanalysis, law, and penology, define certain concepts, such as normality, rationality, insanity, and madness in tandem with practices and institutions (asylums, prisons, hospitals, or sexual codes) that together form a disciplinary society in which a historically contingent and specific set of knowledge/power relations becomes hegemonic and thus dangerous' (Hollinger, 1994 p. 128).

For McGowan (1991), genealogy entails a new description of the intellectual.

Foucault labels the traditional theorist as a "universal intellectual", no matter what the discipline, whether the person works in psychology, economics or marketing. Such a person can delineate the social forces working on society and suggest changes, consequently making sure mistakes are, at best, not made or at least, are corrected (McGowan, 1991). When Foucault analysed the reign of experts in postindustrial society, he came to the conclusion that every production of knowledge serves the interest of some power. "The general, theoretical knowledge produced in 
medicine, economics, psychiatry, anthropology, and the other human sciences since their establishment at the dawn of modernity is part of the power of the social institutions that have grown up around these disciplines; the market, colonial administrations, the university' (McGowan, 1991 p. 125).

In contrast to the universal intellectual, Foucault proposes the specific intellectual whose work is associated with a particular social struggle, in a particular situation with a particular goal in mind. Therefore the specific intellectuals would make no attempt to generalise their conclusions. He is not suggesting the specific intellectual would be aloof from the struggle, acting as some kind of consultant. Rather, he wants the intellectual input to be motivated by the involvement with the struggle.

'There is no power that is exercised without a series of aims and objectives. But this does not mean that it results from the choice or decision of an individual subject; ... the rationality of power is characterized by tactics that are often quite explicit at the restricted level where they are inscribed (the local cynicism of power). Tactics which, becoming connected to one another, attracting and propagating one another, but finding their base of support and their condition elsewhere, end by forming comprehensive systems; the logic is perfectly clear, the aims decipherable, and yet it is often the case that no one is there to have invented them, and few who can be said to have formulated them' (Foucault, 1980 p. 95).

Whilst Nietzsche identified the power relation as an important issue, he did not confine his interest just to political theory. Marx on the other hand, saw power only in terms of the production relation whereas Foucault was interested in the relation between power and knowledge. Power can be viewed from a negative perspective, with reference to a sovereign state, with respect to be able to say no, therefore there is a concern about transgressions. 
Foucault, on the other hand, was interested in the strategic use of power. In this respect he viewed power not as a commodity that can be either seized or acquired, but rather as a part of a network of people where power is applied. For Foucault, power produces reality and knowledge engenders power. It is important to note that power is involved in all social relations. In fact, the individual is constituted by power. Also power is not to be seen as solely negative; it can have positive benefits as well. Foucault sees power etched in the practices of everyday life (Brown, 1995)

There are a number of criticisms of Foucault. Sarup (1993) argues that Foucault does not believe in any ontology or epistemology so the question arises as to how one is to evaluate one local opinion against another? Also, Sarup argues that Foucault postulates the concept of panopticon, which is basically the anonymous centralization of power. But it would seem that power is a metaphysical principle. It is hard to understand what power operates against. There is no emphasis or encouragement to resistance or struggle. Also, there is no constant subject in history so history is uncontrolled and directionless.

Schatzki (1993) also argues that the idea of discourse is obscure as it varies from being a product of language to being part of the social structure, and suggests that everything is a social construction and that there is no reality outside the construction. Another criticism from Schatzki is that Foucault concentrates too much on modernity in terms of technologies and institutions of control and ignores other institutions that have a more emancipatory function. Also, it is argued that Foucault does not specify criteria for deciding what is a right or wrong use of power 
as he believed that it was up to the individual to decide which power relations to either accept or oppose.

This section has outlined the work of Foucault, concentrating on the use of language and also how power is related to knowledge. Whilst there are problems with Foucault's approach, as has been indicated, he does provide some basis for consideration in the marketing context. This is especially the case when viewing the relationships that are developed in the distribution chain. For example, Kasabov (2004) uses a Foucauldian approach to understand shifts in power resulting from technology. He outlines how help lines and direct marketing seek to control and regulate the consumer, and how the consumer reacts to such control.

Notwithstanding the above, the distribution channel is only one aspect of marketing, and given the problems that arise with a Foucauldian approach, it is argued that such a sceptical position does not lend itself to the building of a research programme for marketing management based on the linguistic turn in philosophy.

\section{Conclusion}

The purpose of this chapter has been to explore the vast topic of postmodernism as it is a topic with extremely diverse views on the subject. The framework that was developed as a heuristic allowed for a dissection of the postmodern topic.

In terms of postmodernism as a cultural analysis both Jameson and Baudrillard offer some insights. The question whether postmodernism is a new epoch or an extension of modernism it is too early to say, especially as modernism has had 400 years to 
develop whereas postmodernism is a recent phenomena, being critiqued from the 1960's onwards.

Whilst Baudrillard on final analysis may be seen as too extreme he does identify some cultural tendencies that are worth noting from a marketing perspective. The consumer society is at the heart of postmodernism and terms such as 'retail therapy' and 'shop till you drop' are common sayings (Brown, 1993). Following on from this, it can be claimed that marketing as a discipline is the cornerstone of consumerism. As noted by Firat (1995), marketing is postmodern in practice, at least in the western world and consumers show all the traits of living in a postmodern culture. The consumer has become immersed as an object into the world of objects rather than be divorced from the object as the modernist would have it. The consumer has become a link in the continual production and reproduction of symbolic meaning and images.

And from a marketing perspective much of the postmodern cultural analysis comes as no surprise.

'Baudrillard's suggestion that image is all, that illusion is more important than reality, that fact and fiction are indistinguishable, that image is reality, may well have been a revolution in Marxian thinking, but it is unlikely to disconcert too many marketing academics and practitioners. It is a commonplace, arguably the most basic commonplace, of the contemporary marketing world view, where products are routinely developed to match existing images - cf. Calvin Klein, Ralph Lauren - rather than the other way around' (Brown, 1995 pp. 167-168).

In terms of the aims of this thesis, the philosophical quadrants as presented in Figure 7 (page 138) are of more interest. Both Derrida and Foucault have been discussed and in general terms have been found wanting for a number of reasons, but primarily 
because of their negative or skeptical outlook. As noted by Delanty, (1997) the problem for postmodernism is that by retreating into a culturalist discourse all that is possible is deconstruction of social problems with the result that this is no different from Freyerabend's 'anything goes' position, one that ultimately affirms the existing social order. As indicated by Brown (1993) it would seem that demolition is the only construction that postmodernism recognises.

This chapter has explored the linguistic turn in modern philosophy by exploring the topic of postmodernism. Two dimensions were explored, that of cultural analysis and that of philosophical analysis. Within the strong postmodern quadrant two philosophers were explored, namely Derrida and Foucault, and it was found their philosophies were too nihilistic to build a research programme based on the linguistic turn in modern philosophy. The next chapter will explore in detail the topic of Neopragmatism based on the work of Richard Rorty, and show how it can be linked to praxis. 


\section{Chapter 5: Neo-Pragmatism}

\section{Introduction}

The purpose of the preceding chapter was to explore and make sense of the vast topic of postmodernism. A framework was developed to achieve this purpose by making a distinction between strong and affirmative postmodernism on the one hand, and postmodernism viewed from the standpoint of cultural analysis compared with postmodernism being seen in terms of philosophical analysis on the other hand.

Three quadrants were explored with the work of selected authors explored in each quadrant. The Post-modernity quadrant was explored with reference to Jameson, whereas Baudrillard was used to explain the End of History quadrant. The cultural analysis aspect of postmodernism is important to understand as marketing is the archetypical exemplar of postmodernism (Brown, 1993). Two authors, Foucault and Derrida were used to explore the third quadrant, Nihilism. However, for the purpose of this thesis, that is, exploring the philosophy of science to find a new approach for the generation of marketing knowledge based on the linguistic turn in philosophy, the work of Derrida and Foucault was found to be too narrow and too negative. The task now is to explore an example from the fourth quadrant, namely Pluralism, by exploring Neo-Pragmatism, which too, is based on the linguistic turn in modern philosophy. In fact it is Rorty who coined the phrase 'the linguistic turn in modern philosophy' (Putnam, 1997).

Neo-pragmatism, based on the work of Richard Rorty, was selected to represent the pluralism quadrant as he is a postmodernist who has adopted the linguistic turn in his 
work, and by utilising the work of Gadamer, is able to use hermeneutics to develop the concept of praxis. The thesis argues that neo-pragmatism will allow an alternative research approach to generate marketing knowledge based on the linguistic turn in philosophy, not in an absolute sense, but in effort to promote understanding and insights. Therefore, it is necessary to show why Rorty is an affirmative postmodernist and explore his philosophical position. Rorty has been an extensive writer but his influential book, 'Philosophy and the Mirror of Nature' will be the main source used to extrapolate his philosophical position.

\section{Affirmative Postmodernist}

To explore the Pluralism quadrant it is necessary to first explain why Richard Rorty $(1979,1982,1989)$ is seen as a philosopher who is an affirmative postmodernist.

'The affirmative postmodernists, in their attempt to acknowledge and return a subject to the text, entertain the notion of truth, but a truth as personal and communityspecific. Thus they embrace a form of epistemic relativism in which value choices and representational politic becomes possible' (Carr, 2002 p. 12). Rorty fits the description outlined above as he bridges Continental and Anglo-American philosophical traditions by bringing Derrida, Lyotard, Habermas and Foucault into his thinking. However, the three main philosophers who have influenced his thinking the most are Wittgenstein, Heidegger and Dewey, (1971) who share the idea of historicism but were also interested in practical action. Rorty uses the methods of analytical philosophy to deconstruct the analytic project.

In terms of his postmodern stance, Rorty claims that his philosophy is a complete break from modernism. But in terms of the basic principles of Western thinking, 
Rorty assumes an essential continuity from modernity to postmodern thinking (Dupre, 1993). Whilst not wanting to throw out rationality completely, reliable knowledge is based on the rules of the community rather than some divine rules of truth. What legitimises truth is the praxis of communication. This makes the search for metanarratives superfluous as a search beyond them for legitimation is pointless. Following on from the above, Rorty does not want to dismiss logocentrism altogether. It is one thing to dispense with logocentric metaphysics that searches for some type of essential or foundational truth, but the rationality of speech needs to be preserved. In comparison to Derrida, it is this aspect of Rorty's writings that make him an affirmative postmodernist.

However, it is necessary to explore in more depth so as to understand what Rorty is saying and to determine how his work could lead to a new approach to generate marketing knowledge. To achieve this goal it is helpful to understand his work in 'Philosophy and the Mirror of Nature' at distinct but interrelated levels. Bernstein (1983) claims that Rorty has two streams of consciousness within his writing. One is a metacritique and the other is a rhetorical defense of pragmatism, which states as a bottom line that philosophy is not constructive as there are no foundations for it to build on. Rorty, like Heidegger (1983), Wittgenstein (1974), Foucault and Derrida, wants to end philosophy. Unlike the positivists who put science on a pedestal, Rorty sees the poet and literary critics as the new cultural heroes.

The following themes will be developed and discussed with regards to neopragmatism. The first theme will explore Rorty's critique of the history of philosophy where he traces the influence of Plato and Socrates on western 
philosophy but also shows the impact of Cartesian Dualism, Kant, and then finally his three heroes, namely Dewy, Heidegger and Wittgenstein. The next theme will explore his neo-pragmatism with an emphasis on his ideas of truth, metaphysics and epistemology. The next section will explore the role of Hermeneutics as a means to generate knowledge in relation to rationality. This is in contrast to analytical philosophy where the aim is to establish foundational knowledge based on Cartesian dualism. Praxis is a theme that follows on from hermeneutics, where understanding, interpretation and application are integrated. The last two themes in the chapter outline Rorty's defense of relativism and his view of knowledge generation in the social sciences from a neo-pragmatic point of view.

\section{Analytical Philosophy}

To explore neo-pragmatism in detail it is first necessary to examine Rorty's view of analytical philosophy. His main argument is that analytical philosophy has extolled the virtues of scientific knowledge based on a foundational positivism-empiricism at the expense of other forms of knowledge creation, such as poetry and art.

To understand his position it is necessary to place it in a historical context. According to Dupre (1993) the Greek tradition developed an ontological synthesis which gave culture a coherence regarding its integration with reality. 'When this synthesis began to unravel in the fifteenth century, language and reality, power and dependence, immanence and transcendence separated into oppositional poles. Metaphysics, the traditional reflection upon this unity, came to be replaced by an epistemological search for "foundations" of each of these fragments' (Dupre, $1993 \mathrm{p}$. 294). When thinkers in the twentieth century came to grips with the situation 
different philosophical movements arose, such as logical positivism, existentialism, structuralism, and deconstructionism in an attempt to articulate a consistent position.

However, the above movements were undertaken in a modernist perspective. One basic tenet of modernism is that it views the world in a mechanistic way, in the sense that the world was perceived to be structured and integrated so that cause and effect could be measured. This view meant that positivism-empiricism has flourished, at the expense of other philosophical movements. Positivism-empiricism holds the view that problems are easy to identify and that there are clear-cut solutions. Such a view is dependent on the notion that there is an objective "observer" of phenomena that exists out there in the world. The researcher working within this paradigm is attempting to find out how the system works and identify the true nature of the order of things. According to Rorty (1982) the positivists want to search for the final vocabulary, which is the anchor to all other vocabularies. This can be related back to the Cartesian quest for certainty.

This quest was an attempt to escape from history to find non-historical conditions about nature and the existence of man. In this sense the ambition was to ascertain the foundation of knowledge by determining the basis of knowledge claims and the understanding of the mind. It can be claimed that Locke (1971) was the first philosopher to start this quest by making the distinction between mind and body and stating that the mind was a separate entity. Descartes (1971) carried on the quest by postulating that the mind was a mental substance. It was Descartes who can be credited with what Bernstein (1983) terms the Cartesian Anxiety, a construct that attempts to use reason to find the foundation of knowledge, for example, mind-body 
dualism. Descartes' 'Meditations' portrays a journal of the social being on a quest using meditative reflection to deepen his/her understanding of human finitude. What does it really mean to be limited, finite creatures? Descartes is looking for a fixed point, a stable rock, as a base for human knowledge. This fixed point is not only at a metaphysical and epistemological level but also at a spiritual level. However, it was Kant (1971) that positioned philosophy as a foundational subject, in the sense that it underwrites or challenges the claims of other disciplines. ${ }^{26}$

The claim that philosophy is a foundational subject needs to be seen in connection with the downfall of religious dominance in western society. Rorty believes that the theologians used to be the moral custodians but they were replaced by philosophers. However, scientists took over from the theologians but as they became remote from society, the role of moral custodian has been taken over by poets and novelists and not philosophy, which lost its privileged position in society. In fact, analytic philosophy became more remote from main stream culture so people turned to Oriental Philosophy and books such as 'Zen and the Art of Motorcycle Maintenance', (Pirsig, 1970) as well as dabbling in Indian mystic cults. As Machan,

\footnotetext{
26 A dateline of the historical progression of the main philosophers discussed in this chapter may be helpful:

John Locke 1632-1704 Considered to be the first British empiricist, influencing the development of epistemology.

Rene Descartes 1596-1650 French philosopher who played a significant par in the scientific revolution. He was a major figure in continental rationalism.

Immanuel Kant 1724-1804 German philosopher who was an influential figure of the Enlightenment. His major work was the Critique of Pure Reason, contributing to ideas regarding metaphysics and epistemology.

Martin Heidegger 1889-1976 His work Being and Time is considered to be one of the most influential philosophical books of the twentieth century.

Ludwig Wittgenstein 1889-1951 Worked primarily in logic, the philosophy of mind and the philosophy of language. Both his books, Tractatus Logico-Philosophus and Philosophical Investigations, are highly regarded and influential in philosophy as well as humanities and social sciences.
} 
(1996) notes, in the late 1960s and early 1970s when the western world was in need

of direction and advice, academic philosophy had no advice to offer.

'One way to see how analytic philosophy fits within the traditional CartesianKantian pattern is to see traditional philosophy as an attempt to escape from history - an attempt to find nonhistorical conditions of any possible historical development. From this perspective, the common message of Wittgenstein, Dewey, and Heidegger is a historicist one. Each of the three reminds us that investigations of the foundations of knowledge or morality or language or society may be simply apologetics, attempts to eternalize a certain contemporary language-game, social practice, or self-image' (Rorty 1979, p. 9).

As has been stated, there have been three main philosophers who have influenced

Rorty, namely, Wittgenstein (1974), Dewey (1971), and Heidegger (1983). Each of

these philosophers played a part in bringing to an end the 'Cartesian - Lockean-

Kantean’ philosophical tradition.

'Each tried, in his early years, to find a new way of making philosophy "foundational" - a new way of formulating an ultimate context for thought. Wittgenstein tried to construct a new theory of representation which would have nothing to do with mentalism, Heidegger to construct a new set of philosophical categories which would have nothing to do with science, epistemology, or the Cartesian quest for certainty, and Dewey to construct a naturalized version of Hegel's vision of history. Each of the three came to see his earlier effort as self-deceptive, as an attempt to retain a certain conception of philosophy after the notions needed to flesh out that conception (the seventeenth-century notions of knowledge and mind) had been discarded. Each of the three, in his later work, broke free of the Kantian conception of philosophy as foundational, and spent his time warning us against those very temptations to which he himself had once succumbed. Thus their later work is therapeutic rather than constructive, edifying rather than systematic, designed to make the reader question his own motives for philosophizing' (Rorty, 1979 pp. 5-6).

Wittgenstein, Heidegger and Dewey abandoned a theory of representation as they believed that the mind was not a special area of study. In rejecting the raison d'être of traditional philosophy they presented a new terrain to explore. 
Rorty explores this new terrain by first of all rejecting the notion of essentialism. What is meant by the term 'essentialism' is the task of determining a finite list of characteristics that can be used to ascertain whether something belongs to a defined group. In other words, what would count for something to be an ' $\mathrm{x}$ ', for example, truth or morality? Rossiter's (2001) article 'What is Marketing Knowledge? Stage I: forms of marketing knowledge' is an example of essentialism. His approach is that of an empiricist, based on a form of realism. Rossiter (2001) draws a distinction between declarative knowledge and procedural knowledge, with the development of the former being the goal of the marketing discipline. He attempts to synthesize marketing knowledge into four distinct categories, namely marketing concepts, structural frameworks, strategic principles, and research principles.

However, for Rorty, the attempt to find a finite of characteristics is based on the Cartesian desire to establish the foundation of knowledge. According to Rorty (1982), analytical philosophers want to search for the final vocabulary, which would bet he anchor for all other vocabularies. This can be related back to the Cartesian quest for certainty. But for Rorty there is no god's eye view to allow adjudication between competing knowledge claims, therefore there is no correspondence between our knowledge and the world. Instead there is only a specific situated perspective based on the restraint of habit and context. Following on from this, specific norms and justifiable ends of enquiry are the foundation for rationality within a community of language users. 
This relates to the notion of paradigms. Competing paradigms consequently determine fundamentally different objects of enquiry. This means that Rorty's idea of objectivity is based on his notion of 'final vocabularies', in other words, a paradigm, which in turn leads to the notion of objectivism, whereby an ahistorical, permanent framework can be used to determine the nature of rationality, truth, and reality. Rorty challenges this view and rejects the objective/subjective dichotomy as it is designed to parallel the fact and value distinction developed by the positivists. According to Rorty (1989) it would appear that the distinction creates more difficulties than it resolves.

Therefore it follows, for Rorty, that any talk about metaphysics is illegitimate and foolish. He believes that it is necessary to return to Socrates and learn to listen, talk and weigh the consequences of what is discussed. This activity involves moral virtues and has nothing to do with epistemological or metaphysical quests.

In this section Rorty's argument against analytical philosophy has been explored. In summary, Rorty argues against Cartesian dualism and its quest to find foundational knowledge. In particular he is against the notion of essentialism as there are no ahistorical characteristics of any knowledge claims. In other words, there is no final vocabulary.

\section{Neo-Pragmatism}

Rorty believes that a different view of philosophy is needed as modern philosophy fails to come to grips with aspects of people's lives that really matter. Philosophy has lost its prime position as many of the topics and issues that used to be its 
province have been taken over by other disciplines or specialised sciences. Rather than having a determining role in other disciplines, Rorty believes philosophy should play a subservient role. It should be an educated conversation that people can take part in.

Therefore, Rorty wants to incorporate the linguistic turn and postmodernism into his exposition on the history of philosophy. Rorty's different view of philosophy is based on neo-pragmatism. Whilst Richard Rorty has brought neo-pragmatism into the limelight other major philosophers have also given credence to the school of thought, namely, Hilary Putnam and Richard Bernstein, both American philosophers.

Unlike analytical philosophy, it is not based on theoretical notions or about discovering truth. Instead, it is about a way of doing philosophy. However, classical pragmatism, as distinct from neo-pragmatism, was popularised in early nineteenth century by Charles Sanders Pierce, William James and John Dewey. The earliest notions of classical pragmatism were based on the view concerning respect for others and that conversations were more important for developing beliefs than the search for truth, which often involved dogma.

However, 'pragmatism' could be classed as one of the most misunderstood terms within philosophy. The everyday use of the term 'pragmatism' has been taken to mean a person who is practical minded and has no recourse to theory. A pragmatic approach is where the emphasis is on the practical consequences of an action. This is in contrast to the philosophical use of the term, where a person is willing to listen to 
many different versions of the "truth" so that problems can, in some sense of the word, be solved.

Building on Classical Pragmatism, especially the work of James, Rorty takes a sociohistorical approach and rejects the notion that it is possible ever to know the answers to the classical philosophical questions. Therefore he is not interested in questions such as the relationship between language and reality, or what being qua being might encompass, or what counts to be a human being. He believes that gaining a broad overarching understanding of the way the world is constituted, especially about the nature of reality, is beyond our reach. This theme, that is, a distrust of human reason, is apparent in most postmodern writing. For Rorty, what counts as rational is dependent on different intellectual communities.

According to Rorty (1989) there are two senses of rationality. The first is when rationality is equated with method in the sense that criteria are determined in advance and the task is to work methodically through to a solution. Law and medicine are good examples of this approach but natural science is the quintessential example. However, another sense of rationality can be taken to mean 'sane' or 'reasonable'. For someone to be rational in this sense is to be able to discuss any topic which arises without resorting to dogma, ideology, or self-righteousness. Rorty claims that it is this second sense of rationality that needs to be adopted. 'We should avoid the idea that there is some special virtue in knowing in advance what criteria you are going to satisfy, in having standards by which to measure progress' (Rorty, 1989 p. 9). 
Consequently, it is not a matter of justifying knowledge in terms of the relationship between the subject and reality as knowledge generation is a social phenomenon. So there is no court of appeal or something stable that can be consulted for final arbitration but only the community to which you belong. What is needed is coherence to norms and standards of particular rationalities. Consequently true or false statements are dependent on whether they confirm or contradict the rules of the community.

Therefore, the task is to take conversations seriously without asking for 'rational consensus' or to posit the inquiry as a means of finding the so-called 'truth'. It weaves together theory and action to determine beliefs that clarify meaning and allow action to be seen as applicable for a given situation in a given context. This means that theory and action are intertwined, each modifying the other whilst at the same time maintaining their mutual relevance. Rorty believes that we must accept the radical contingency of social practice that defines what we are, so the task is to develop a coping strategy by opening up dialogue and conversation. Therefore, a practical-moral vision drives his work.

As discussed in the previous section, Rorty blurs the distinction between objective and subjective as well as fact and value. Instead, he leaves room for alternative narratives and promotes intellectual tolerance. What this means is that, at a basic level, what is considered is a course of action and its observable consequences. Furthermore, it is the sum of these consequences that forms the meaning of the action. Therefore, pragmatism is a method for evaluating philosophical problems by 
working through the practical consequences. Creation and construction is privileged over discovery and objective description.

In summary, neo-pragmatism rejects foundational knowledge but retains a notion of rationality, which is in contrast to strong postmodernists. However, Rorty's rationality is based on particular situations. Therefore, what is important are particular actions and their consequences.

\section{Hermeneutics}

The previous section outlined how Rorty rejects analytical philosophy and rejects the need for epistemology, as it is concerned with finding the foundations of knowledge. By rejecting epistemology Rorty collapses theory and practice, and argues for a philosophical approach based on practical reason and its consequences. However, if epistemology is replaced, the question arises as to what will take its place. Rorty adopts hermeneutics as the basis for understanding the world.

Hermeneutics is concerned with not only finding meaning in actions, be it an individual or an organisation, but also in developing understanding. Hermeneutics involves the interpretation of not only overt signs such as conversation and texts, but also non-lingual expressions, for example, body language, so that an understanding of tacit knowledge is gained (Gummesson, 2003). The art of textual interpretation has its origins in jurisprudence and theology. When interpreting complex constructs, such as symbolic representations, the relative situational and historical context needs to be taken into account. However, the researcher's own historical and situational 
context must also be acknowledged as it influences any translation or interpretation (Steffy \& Grimes, 1986).

Also, hermeneutics opposes scientism (Bernstein, 1983). As has been noted, scientism is a modernistic penchant to view the natural sciences as a base for all areas of experience and enquiry, and is normative to all knowledge generation. The overarching goal is to find ahistorical concepts that apply in all possible worlds. But hermeneutics challenges the proposition that scientific inquiry is the only means of knowledge generation.

According to Carr, (2002) there are three basic types of hermeneutic approaches, as follows. The first is methodological hermeneutics, which claims that the social sciences require a different methodology from the natural sciences consequently it is impossible to 'objectify' the social world. There is a difference between knowledge derived from explanation and knowledge derived from understanding, with the latter requiring different methods. An example of this type of hermeneutics is work by Hatch and Rubin (2006), whereby they develop a hermeneutic theory of branding, which allows them to trace how a brands meaning changes over time. Such an understanding allows managers to increase the brand's potential.

The second type, critical hermeneutics, is associated with the work of Habermas (1977), where he claims that method and ontology are not sufficient in themselves to explain social behaviour. Habermas made a critique of Gadamer, based on a Hegelian-Marxist tradition, as he believed it lacked an explicit critical function. He was also sceptical of the universalistic claims made by hermeneutics. He argues that 
an adequate social science theory must involve an interpretative or hermeneutical dimension. From his point of view what is needed is a theory of communication competence as social interactions are mediated through language and is a means to determine ideology and types of domination and repression. 'Against Gadamer, who intended to contrast scientific method with the hermeneutical phenomenon, Habermas argued for the necessity of a dialectical synthesis of empirical-analytic science and hermeneutics into a critical theory that has a practical intent and is governed by an emancipatory cognitive interest' (Bernstein, 1983 p. 43).

Habermas wanted to include the notions of work and power, as well as Gadamer's notions of language and communication, as part of a social theory. In developing his social theory Habermas attempted to create a transcendental standpoint. This is in contrast to Rorty who claims that a permanent framework is not needed.

The third type is that of ontological hermeneutics is where understanding is seen as part of being in the world. In other words, ontological hermeneutics refers to the nature of human beings, where one is always in a mode of attempting to understand (Arnold and Fischer, 1994). One of the early philosophers to draw attention to the ontological significance of hermeneutics was Heidegger. He claimed that hermeneutics was universally applicable. Heidegger believed that people were 'thrown' into the world and are always working towards their future and in doing so, human beings are a species who understand and interpret. Therefore it is necessary to understand 'understanding' as it pervades all our activities. 
Heidegger ${ }^{27}$ captured the word 'Dasein' to articulate what being in the world meant. Hermeneutics is an interpretation of Dasein as words show something beyond themselves, namely 'being' that needs to be comprehended. Richard Rorty (1979), following Gadamer (1975), bases his neo-pragmatism on ontological hermeneutics.

Within ontological hermeneutics there are three stages, namely preunderstanding, understanding and application (Bernstein 1983). Preunderstanding is about the knowledge that is brought to the research project before the enquiry begins, therefore, the researcher must acknowledge that they are a historically produced entity with certain biases. Understanding arises from the result of the research so the context and situation of the data collection must be considered. Hermeneutics is not trying to explain how the world operates but is attempting to grasp an understanding of social problems and explain how they can be solved. In this view, the individual constitutes what is in the world. Understanding is pre-judged, in other words, social actors have prejudices that they bring prior to understanding any problem. So knowledge does not grow but people understand differently. 'Hermeneutic understanding is in part self-understanding, self-reflection, and self-development' (Arnold and Fischer, 1994 p. 59). The openness of the future means that it is impossible to know for certain. Over time actors try to make sense of what has been experienced. 'We understand differently but remain uncertain. Learning does not remove uncertainty about what will happen in the future, nor does it remove doubt about what has happened as the past is always a matter of interpretation' (Addleson, 1996 p. 34). The final stage, application, involves practical judgment but in doing so

\footnotetext{
27 Heidegger was interested in an analysis of being [Sein] and found that it was not exclusively logical or on the other hand consisting of cognitive matter, unlike the position taken by analytic philosophy. From Heidegger's perspective, being needs to be analysed through an existential analysis of human being [Dasein]. By ontological reflection one is confronted by the issue as to what it is to be distinctively human.
} 
the researcher needs to think about the relationship between theory and history, in so much as any theory is contextually based (Steffy \& Grimes, 1986).

For Gadamer, interpretation is involved in hermeneutics and the notion of interpretation is based on some beliefs about ourselves. Human beings are able to pre-conceive issues. Such prejudgement is based on the idea that we are beings engaged in interpretative understanding. The recognition of our prejudices enables us to understand and hermeneutical understanding is constitutive of what we are in the process of becoming. For hermeneutics, there is no distinction or difference between understanding and interpretation.

The above discussion raises a question about the concept of prejudice. For Gadamer, prejudice needs to be seen as 'prejudgement' rather than something that is negative, unfounded and false. All knowledge involves prejudgements and as such, these must be tested. There are two basic types of prejudices, namely blind and enabling, and they can be determined by art, texts, and traditions (Bernstein, 1983).

Prejudices give rise to the notion of "belongingness", which is like having an affinity with something. Humans are always influenced by our being 'thrown' into our world. ${ }^{28}$ This links to the issue regarding traditions. It is traditions which shape us and come alive when freely appropriated. All understanding is projective and is based on three important fields, namely tradition, now, and the future.

\footnotetext{
${ }^{28}$ The notion of 'thrown' comes from Heidegger in 'Being in Time'. He makes the point that as humans we are thrown into the world and act as Das Man, meaning inauthentic existence until we are confronting by our angst where we have the opportunity to confront our future and become authentic individuals.
} 
According to Bernstein (1983), two important concepts arise from the above discussion. First is the concept of the effective-historical consciousness and the second is the fusion of horizons. The former relates to prejudices and how they are linked with tradition, whereas the latter is about the ranges of vision that is possible, which is dependent on one's standpoint. Such a vantage point is finite and limited but it is possible to move beyond it. This means that one is never bound to one standpoint as it is always possible to move to another vantage point. Fusion happens when one tries to understand another horizon. When this happens one becomes both endorsed as an individual and enriched, but at the same time testing one's prejudices.

As has been intimated in the above sections, the concept of understanding is important for Gadamer (Bernstein, 1983). He believes that nothing is, in principle, beyond understanding. It is the basic mode of being, having ontological status and also being a universal concept. There is a link between understanding and language. It is through language that we articulate or comprehend our understanding. Language is the medium in which we live so is not just an instrument that is used. Following on from the above, Gadamer's aim is to expose the false dichotomy of thinking in terms of objectivism and relativism and show how our views are distorted when this dichotomy is imposed on our thinking.

As a starting place to articulate his line of reasoning, Gadamer concerned himself with the subjectivism of aesthetics. Specifically he questioned the notion that there was no truth in art. Why did art and beauty have nothing to do with knowledge and truth? To put the issue in its historical context, it is necessary to begin with Kant's 
concern with the topic of aesthetics. He wanted to develop the same understanding for aesthetics that he had developed with his work on a priori knowledge and morality. By using the notion of aesthetic consciousness he was concerned about reflective judgement and to ascertain the a priori foundations for aesthetic judgements. He noted that such judgements were grounded in human subjectivity but that does not mean that they are relative to the individual. In other words, taste, for example, is communal (Bernstein, 1983).

However, ironically for Kant, grounding aesthetics in human subjectivity lead to aesthetics being part of personal preferences. Gadamer, on the other hand, wants to overcome this bias. To achieve this ambition, he uses the concept of play and gives it an ontological status. He makes the observation that when one is involved with play one loses oneself. One becomes involved and absorbed. In the same respect, one becomes involved with art. There is a dynamic interaction between the piece of art and the perceiver so that one shares in it. The interpreter is not detached but the work of art makes a claim. Does this lead to relativism? Gadamer would say 'no' as he rejects such dichotomies (Bernstein, 1983).

An important concept within hermeneutics is that of the hermeneutical circle, which claims that in order to 'understand' one must 'foreunderstand', and this involves anticipation and contextualization. One can only know what one is prepared to know. Therefore, the hermeneutical circle is about understanding the whole of the text by the parts of the text. There is a movement back and forth between the parts and the whole. In other words to understand an issue or problem one goes from local 
detail to global structure. One is able to validate the circle, that is, the whole, by an appeal to the parts ((Bernstein, 1983).

This approach is in contrast to the positivists who want to break out of the circle and find a touchstone so that it can be determined which or what readings of a text are correct. But the hermeneutics position argues that there can be no empirical verification based on 'superficial' data.

Given the above outline of hermeneutics, it is now appropriate to explore how Rorty uses it to develop his thesis. He sees hermeneutics as an attempt to put aside the idea that man has an essence, and that it is possible to mirror that essence through knowledge of the world with a master vocabulary which allows commensuration of all discourses.

Hermeneutics is a fact about people, rather than some enquiry as to what constitutes truth. It is a process of redescribing ourselves and this is achieved when reading, writing and talking take place. Education and self-formation supplants 'knowledge' as the goal of thinking. 'From the educational, as opposed to the epistemological or the technological, point of view, the way things are said is more important that the possession of truths' (Rorty, 1979 p. 359).

Rorty uses his term 'edification' as the project to find better, more fruitful discourse. Therefore, abnormal discourse is an aid to become new beings. Consequently, the search for objective knowledge is only one project among many rather than the only project. The assumption that there is only the one project can hinder the project of 
edification. Science does not have a privileged attachment to reality which makes it more than just a set of descriptions. Therefore, science is on the same footing as poets, novelists and artists.

Rorty makes the claim that there are two sorts of philosophers. Systematic philosophers believe that knowledge is based on justified, true belief but for edifying philosophers such an approach leads to conformity. 'The mainstream philosophers are the philosophers I shall call "systematic", and the peripheral ones are those I shall call "edifying". These peripheral, pragmatic philosophers are sceptical primarily about systematic philosophy, about the whole project of universal commensuration' (Rorty, 1979 p. 367). Rorty cites Dewey, Wittgenstein and Heidegger as being examples of edifying philosophers.

\section{Praxis}

Given that the main thrust of hermeneutics is understanding, it is now necessary to see how the concept relates to Praxis. Understanding is linked with interpretation and application and the three terms are integrated, forming the basis of phronesis. Knowledge, therefore, is not detached from the observer but is a constituted part of his/her praxis. According to Aristotle (1970), in the Nichomachean Ethics, there are three intellectual virtues. The first, episteme, is scientific knowledge, which consists of deduction from basic principles. 'Episteme is highly self-contained because it is deployed mainly in theoretical discourses themselves. Although episteme obviously is not as self-contained activity, it aims to remove as many concrete empirical referents as possible in order to obtain the status of general truth' (Greenwood and Levin, 2005 p. 50). This means that episteme is closely linked to theory 
development. The second virtue is techne, which can be considered as craft knowledge, in other words, how to make things. The task is to make better designs for living, thus increasing human happiness. The third virtue, phronesis, is practical wisdom which involves knowing what is good for human beings in general and applying such knowledge to particular situations. According to Tsoukas and Cummings (1997), Aristotle views phronesis as the highest intellectual virtue.

Praxis is concerned with activity and based on Aristotle's distinction between praxis and phronesis, where the former is about action in a particular situation and the latter is about morals dispositions, which directs praxis (Bernstein, 1983).Therefore, praxis involves the interaction of judgement and action.

According to Bernstein (1983), praxis allows for the mediation between the universal and the particular, involving practical judgement which involves deliberation and choice. Praxis involves choice and judgement about practical issues in concrete situations. This leads to the notion of informal action, which is the ability to coherently explain and understand situations where judgements are made. In summary, we are dialogical beings, always in conversation with either ourselves or other beings, concerned with the process of understanding.

Tsoukas and Cummings (1997), outline three reasons as to why practical wisdom important? First, marketing decisions change over time, therefore new problems will require flexible and imaginative responses. Applying rules and guidelines that were useful in the past does not guarantee success in the future. Second, practical matters are indeterminate and ambiguous, so the solution will depend on one's purpose. 
Finally, often each decision is unique so the decision maker needs to treat the case accordingly. Therefore, each act is dependent on a given context. 'The actor needs to make sense of the context to enable appropriate actions' (Greenwood and Levin, 2005 p. 51). Consequently, praxis, that is, practical judgement, does not occur independently, but is an integral part of a given situation.

\section{Relativism}

The chapter so far has outlined Rorty's criticism of analytical philosophy, and in its place postulated neo-pragmatism, whereby practical reasoning and its consequences are of importance. By adopting hermeneutics, Rorty is able to develop the notion that understanding is central to our being human, and the notion of praxis is an important concept regarding how human beings should conduct themselves. However, one criticism of Rorty's position is that of relativism.

However, it is necessary to note that relativism is different from subjectivism. The former utilises pluralist approaches and does not necessarily have to be subjective, whereas the latter concept relies on transcendental philosophy with a priori postulates. For the relativist there is no overarching metalanguage. Therefore it is impossible to evaluate or adjudicate between competing concepts or paradigms. There are no universal standards that are applicable in all possible worlds. However, it can be claimed that relativism itself may be true or false, consequently it is impossible to claim relativism without undermining it as it is a foundational statement. How can it be possible to escape this paradox? The solution from a pragmatist point of view is not to make the claim in the first place. The dichotomy 
makes sense only if the Cartesian dilemma makes sense. What would it mean not to accept it, and move beyond it?

In defence of his position Rorty makes the point that relativism is not that any view is as good as another. Obviously this is absurd when taken to its logical conclusion. The second form of relativism is where 'true' is an equivocal term having as many meanings as there are contexts of justification. According to Rorty this is a 'wrongheaded view'. The third type is that of the ethnocentric view, where nothing can be said about truth or reality apart from descriptions of the familiar procedures of justification which a given society uses in an area of enquiry. His view is that relativism is about culture, purpose or intuitions being supported conversationally.

"Relativism" is the view that every belief on a certain topic, or perhaps any topic, is as good as every other. No one holds this view. Except for the occasional cooperative freshman, one cannot find anybody who says that two incompatible opinions on an important topic are equally good. The philosophers who get called "relativists" are those who say that the grounds for choosing between opinions are less algorithmic than had been thought. ...So the real issue is not between people who think one view is as good as another and people who do not. It is between those who think our culture or purpose, or intuitions cannot be supported except conversationally, and people who still hope for other sorts of support' (Rorty, 1980 p. 736).

Rorty reduces objectivity to solidarity, therefore metaphysics and ontology are not needed to explain the relation between beliefs and knowledge as required by a correspondence theory of knowledge. 'From a pragmatist point of view, to say that which is rational for us now to believe may not be true, is simply to say that somebody may come up with a better idea' (Rorty, 1989 p. 12).

According to Bernstein (1983), the goal of inquiry is to ascertain the application and usefulness of beliefs, rather than trying to determine truth. Therefore a variety of 
voices adding to a narrative give rise to vitality and meaning. This involves a tolerance for all viewpoints and truth then is a matter of belief. This means that in evaluating beliefs it becomes a matter of moral values rather than specific criteria as to what would count as knowledge.

Therefore, it can be seen that Rorty accepts that his position is based on a form of relativism, but if there is no foundational knowledge, such a position is the only tenable one.

\section{Social Sciences}

Rorty's position now leads to a discussion of science and the social sciences. Terms such as 'science', 'rationality', 'objectivity', and 'truth' are all intertwined. Rorty (1989) sees scientists as the High Priests of modern society. It is to scientists that people turn when they have problems in their lives that need solving. It is the conception of science as dealing with the hard objective truth that people cling to, where it is assumed there is a correspondence to reality. Objective truth is based on reason so must be accepted as the right explanation. But it can be seen that Rorty rejects the notion of a correspondence theory of knowledge and the notion of objective truth.

From a historical perspective the development of theory within science meant that choices had to be made between what counted as science and what did not count as science. This choice poses an interesting dilemma for Cartesian thinking. The issue is whether there are clear and explicit criteria for demarcating science from nonscience? Based on an either/or dualism, the question demanded from Cartesian 
thinkers is providing a philosophy of science that is able to determine fixed criteria. The choice facing Cartesian thinkers is to choose between objectivism and relativism. However, Kuhn challenged this type of thinking. He is basically saying that there are no fixed criteria (Lovitt, 1997).

Rorty notes that quantitative analysis uses hard data to match conceptual frameworks and so consequently is based on an empiricist dogma. The idea of data and concepts linking up to give sense to a theory of knowledge is unnecessary and should be dispensed with. Knowledge is generated through a process of edifying, pragmatic discourse based on various disciplines, where judgement is suspended, novel ideas are discussed and the ultimate choice of one approach over another is based on expediency.

However, he does note that science is a useful method of inquiry and provides ends that work but it should not be seen as the only vocabulary that can be used to generate knowledge. The scientific method should not denigrate other vocabularies that are useful in pursing different ends. He believes that science is only a vocabulary for coping, but it is a trap to think it is the only one as it is too limiting in terms of knowledge generation.

What Rorty is saying is that science as a community is a model of human solidarity. Therefore he is not debunking science as a method but only its place as the highpriest of our culture. However, Rorty notes that given the social acceptability of the scientific approach, other disciplines attempt to adopt the same 'scientific procedures' to gain respectability. He comes to the conclusion that the social 
sciences would be better off not to emulate the natural sciences as they are interested in predicting and controlling the behaviour of things, whereas the social sciences may be better to develop understanding rather than explanation.

Rorty suggests that certain argumentative stalemates should be set aside. Attempting to obtain theoretical consensus and closure is misguided. What is needed instead is inventive new views and critique of practice. 'To the extent that the Western philosophical tradition has been dominated by the privileging of such rhetorical values as argument, rationality, consensus, and power, it is necessary to circumvent, or set aside, such a tradition to escape its spell' (Horne, 1993 p. 170).

Therefore knowledge generation takes on a new hue. 'It is by the use of circumvention of argument that Rorty emphasises images, metaphors and neologisms as a basis of edifying conversation' (Horne, 1993 p. 171). This approach is based on several key concepts. First, as mentioned, edifying philosophy is promulgated instead of systematic philosophy. To attain this dimension the driving force is figurative rather than epistemological so that philosophical writing involves irony, satire, and aphorisms. Such an approach is achieved through conversation rather than argumentation, so solidarity is more important than consensus. What we may believe now may not be true in the future as somebody may come up with a better idea. We are continually reweaving a web of beliefs rather than the application of criteria to causes. Criteria change in the same way some beliefs change so therefore all criterions are open for revision. By understanding Kuhn, the story of progress can be traced with a certain direction but it does not mean that a goal is out there waiting for us. As Rorty notes 'the end of human activity is not rest but rather richer and 
better human activity' (Rorty, 1989 p. 14). Finally, alternative vocabularies need to be generated so that there is a process of redescription. According to Horne (1993) this is a key concept as it is a means of self-creation as well as a means to generate political debate and obtain social change.

The notion of redescription is based on the work of Dewey, Heidegger and Wittgenstein. 'They hammer away at the holistic point that words take their meanings from other words rather than by virtue of their representative character, and the corollary that vocabularies acquire their privileges from the men who use them rather than from their transparency to the real' (Rorty, 1979 p. 368). It can be seen that Rorty adopts an ethnocentric view. He interprets this as 'is simply to work by our own lights. The defence of ethnocentrism is simply that there are no other lights to work by' (Rorty, 1989 p. 11).

\section{Conclusion}

This chapter has explored the work of Richard Rorty, an affirmative postmodern philosopher who is an example of a writer that fits into Quadrant Four. The question now arises as to whether neo-pragmatism provides a platform for the development of a new research approach for the marketing management school based on the linguistic turn in philosophy? The answer to this question is yes, for the following reasons.

First, neo-pragmatism is an affirmative postmodern thesis in the sense that it does endorse reason. It is reason that is used to enable discourse and understanding. This is in contrast to positivist- empiricism that seeks truth as the ultimate goal and the 
nihilistic philosophers in Quadrant Three who see reason, that is, rationality, as a modern phenomenon. Rorty wants to preserve a form of rationality, but not in a foundational sense, but as a means of enabling discourse regarding particular actions and their consequences, so as to develop understanding.

Following on from the above, Rorty makes the point that truth is attainable in the sense that it relates to a specific context and historical epoch. Following Wittgenstein's form of language, Rorty holds that truth belongs to the group who are undertaking a particular language game at the time. Therefore there is no God's eye view of the world where truth is the ultimate goal in a theory of knowledge.

This leads to an important issue for marketing. That is, Rorty's anti-foundational stance. Rorty applauds many voices and many ways of viewing issues. Therefore this allows for the generation of new perspectives based on abnormal discourse. What he means by abnormal discourse is that such talk is incommensurate and therefore is creative and challenging. What Rorty means by the term 'incommensurate', following on from the discussion in Chapter 2 on incommensurability, is a new approach to a topic using new language which challenges the existing form of language. This for Rorty is exciting as it allows for new ideas to be discussed and on-going conversation to take place.

An important issue as an outcome of ontological hermeneutics is the emphasis on praxis. The notion of practical wisdom has relevance for the area of marketing management, with its emphasis on choice and decision making. 
Finally neo-pragmatism has an emphasis on discourse. This allows for different voices to be heard and new understandings to be voiced. It is through such edifying processes that new knowledge can be generated. There is no one correct way to generate knowledge under neo-pragmatism. It is more a discussion on different perspectives and the adoption of the most expedient solution given the context and history of the situation.

The above constructs can be used as a basis to move forward to develop a different approach to generate marketing knowledge. The next chapter will develop Narrative Theory, based on hermeneutics, as an interesting way to view marketing issues. It needs to be remembered that in developing a new research approach, it is not being claimed that it is the only 'right' way to conduct marketing research. What is being put forward is an alternative approach to generate marketing knowledge, not in an absolute sense, but in an effort to promote discussion and understanding. 


\section{Chapter 6: Narrative Theory}

\section{Introduction}

The argument of this theoretical thesis is that marketing management should adopt the linguistic turn in modern philosophy. The thesis has justified this claim by discussing problems regarding logical empiricism and issues surrounding postmodernism. It was then argued the marketing management would benefit by adopting the affirmative postmodern perspective of Richard Rorty to provide a basis to develop a new way of generating marketing knowledge. After reviewing his work, four main points were made. First, he emphasises the linguistic turn within philosophy. Second, he rejects Cartesian dualism and following on from this adopts an anti-foundational approach to the philosophy of science. Third, Rorty claims that a concept of truth is dependent on specific situations and historical epoch, and finally he is concerned with praxis and how it can be developed. This latter point highlights the importance of discourse and context when undertaking marketing research.

The question arises as to the reason narrative theory was chosen as a methodology? It needs to be remembered that Rorty does not believe that science is in a privileged position in terms of knowledge-generation. He believes that other forms of inquiry, such as literature and films, are equally valid ways to gain knowledge. But this is not the only justification for the adoption of narrative theory as a methodology. Another important reason is the narrative form is an excellent way for researchers to understand practical thinking. ${ }^{29}$ 'Narrative thinking involves the building of a

\footnotetext{
29 This is not to say that there are other methodologies that could be adopted for undertaking practical thinking, for example, discourse theory. However, narrative theory allows the story teller to
} 
convincing story which attempts to show the coherence between the actions of the individuals involved in a particular situation and the meaning of the situation for them' (Tsoukas \& Cummings, 1997). Also, narrative theory allows for multiple voices to be heard and complex situations understood. A further reason why narrative theory is appropriate is because hermeneutics began as a study of texts (Paton, 2002), and narrative theory extends the idea of text to incorporate in-depth interviews and stories based on life history. Hermeneutics, with its emphasis on interpretation and context (Patton, 2002) is part and parcel of narrative theory.

Narrative theory is a method that addresses the above points. In other words, neopragmatism provides the ontological and epistemological framework, with narrative theory flowing on as a method. Narrative theory postulates that people's understandings and experiences are understood through stories. Therefore this chapter will explore narrative theory as a method to explore issues in marketing management. The chapter will begin by exploring issues surrounding narrative theory, which will then be followed by outlining the core constructs of narrative theory. The chapter will explore postmodern narrative theory and outline eight ways to analyse narratives. Finally, the chapter will explore the link between narratives and sensemaking within organisations.

\section{Background}

Narratology is the theory and systematic study of narrative (Currie, 1998). It began in the early twentieth century by the structuralists and Russian formalists. In the late

explain the interaction between their actions as an individual and their meaning of the situation for them. 
1980 's there was a rebirth of narrative theory and the scope of the discipline was widened to include film, advertisements, songs, comic strips and even jokes (Fulford, 1999). At the same time other academic areas outside of literary criticism, such as sociology, psychology, economics and philosophy were also utilising narrative theory. Currie (1998) cites three main thrusts for the widening scope of narrative theory. The first thrust was that of diversification, which saw the transition from poetics to politics. The second thrust was that of deconstruction, where scientific authority was challenged and dismissed as the only basis for knowledge, and new methods for unmasking ideology in society were developed, for example, the approach to binary opposition where one view is privileged over another. The final thrust was that of politicalisation of narrative theory with an emphasis on complexity rather than coherence.

The study of narrative continues to grow and is found in a greater range of disciplines from philosophy to law and the social sciences in general (Richardson, 2000). The question is, however, about the relationship between narrative theory and the social sciences. Are they not two mutually exclusive activities? Social scientists discover whereas writers create; social scientists observe reality whereas writers invent different realities. However, the similarities between the two acts are striking. 'On closer examination, we find that social scientists often do what writers do: they create rather than discover, they focus on the unique and individual, and they use illusion and rhetoric in an effort to make their case. Similarly, writers often act like social scientists: they test ideas against evidence, they generalise, they pose testable questions about the social world, and they try to remain faithful to details of external experience' (Phillips, 1995 p. 627). 
Therefore, it is not that there are two distinct communities but two loose communities who have the same goal in mind, that is, both are attempting to model the world, only separated by the conventional techniques of their respective activities. It is not that there is a gap between the two activities, where one finishes and the other starts.

'Increasing the diversity of the ways we approach the world of organizations increases the complexity of what we find there: each perspective reveals a new set of social objects and a new set of possible relations' (Phillips, 1995 p. 628). Therefore, opening up marketing discourse to narrative theory allows the discipline to move in new directions and new facets can be explored. However, it needs to be remembered from a philosophy of science point of view that what has been postulated is an antifoundational view, so any new directions are not bringing us closer to a final representation of the world. Rather, each new view shows a new aspect of marketing, adding a new perspective to the discipline. 'Many different viewpoints can be included in the text, each represented by a character. No final sense of closure needs to be created and none is implied by the presence of the omnipotent author of traditional research reports' (Phillips, 1995 p. 629). However, the trade-off is that this gives rise to doubt, uncertainty, and paradox which is less of an issue than with traditional social science approaches. 


\section{Work to Date}

Much of the work on narrative theory within the social sciences has been in the areas of anthropology and sociology. However, over the last ten years there has started to be some interest in applying it to management studies. This section reviews the main contributions in the management and marketing area.

Brown (1998) uses narrative theory as a method to understand actions and events within an organisation. He explores a group's interpretation of an IT implementation solution and by understanding the stories that were told it was possible to see how members of a group gained understanding and obtained meaning of an event. The study was able to identify how individuals gave legitimation to their ideas in their attempt to make adjustments to a new IT environment.

Schwartzman (1984) studied the stories that were told in a dysfunctional community health centre. She classified three types of stories, namely those about meetings, stories about individuals and stories about the organisation.

Boje (1995) uses postmodern analysis to explore a storytelling organisation, namely Disneyland, to resituate excluded stories and voices in comparison to the dominant legend of the organisation, which is portrayed as happy and profitable. The aim was to explore the organisation by analysing the use of language, including texts and discourse. By using a discursive metaphor to analyse a contextualised network, he moved away from the usual mechanistic and organic metaphors. This in turn means that the understanding of events depends on the locality, the sequence of the story and the effect on the characters telling the story. 
Beech \& McCalman (1997) explored the narratives used by teams in two high-tech organisations. The use of symbols and stories was found to reinforce better buy-in by members and strengthen the team culture. However, narrative analysis can also be used to monitor discord within the teams and the level of commitment of its members.

Boje (1991) undertook a participant observation study in an office supply company. He was able to listen to stories told by employees as they went about their business on a day-to-day basis, consequently capturing what was happening in the natural context of the situation. This is in contrast to self-report retrospective stories that are told outside of the context. Boje was able to observe how stories are told to make sense of events and change within the company, as well as gain political advantage. The study supports the view that an organisation is bound together by a system of story-telling and such stories become an inherent part of the company culture.

Bush, Harris \& Bush (1997) suggest that the narrative paradigm as developed by Fisher (1984) ${ }^{30}$ be used to explore ethical dilemmas in the service industry. The benefit of this approach is that situational and individual characteristics can be taken into account whereas the empirical/rational perspective has not had much impact in developing ethical research in the marketing arena.

A seminal paper, published by Barry and Elmes (1997) used narrative theory to explore strategic management as a form of fiction. They were interested in the way

30 See page 196 for a further description of Fisher's Narrative Paradigm 
that stakeholders use language to create direction and to motivate actions. They believe that narrative is a means to capture the diversity and complexity of strategic discourse. It also highlights how strategy in organisations is a discursive practice, linked to cultural and historical contexts. For Barry and Elmes, narrative provides an opportunity to open up new trains of thought and offer a different perspective on strategic thinking. If story telling is the main method people use to make sense of their situations, the task of the strategist is to tell a story that is convincing so the stakeholders buy-in to the strategy. The context and how the story is told is important. 'How employees and managers engage in strategic story making - what they borrow and reject from mainstream thinkers and how they make sense of the process as they go - has implications for understanding both what makes a strategic genre credible and fresh and how it does or does not help individuals to navigate the murky waters of postindustrial organizational life' (Barry and Elmes, 1997 p. 447).

Boje et al (1999) used stories to analyse the power relationships between members of a choir. The interesting point about this research is the methodology that was employed as they used students to conduct the interviews and their stories about the interview process were also included in the research, along with participants' stories. The participants also had a chance to comment as a group on the transcripts of the stories that the researchers had told. The hope of Boje et al was to be more reflective about their writing and not to fall into a hegemonic trap. They were able to capture polyphonic voices that were fragmented and did not fit into the whole picture without narrative editing. 'The postmodern turn is to leave the allegory in its fragments and be content with the differences. The report of our stories is a muddy and murky process. Being self-reflexive is a process of continually discovering that we, as 
writers in this endeavour, are shaping the stories and character of our subject' (Boje et al, 1999 p. 18).

Shankar and Goulding (2001) outline narrative theory as a possible interpretive method for consumer research. 'We are proposing that consumption experiences, and other marketing related phenomena, can be interpreted narratively' (Shankar \& Goulding, 2001 p. 9). The authors go on to say that the method lends itself to understanding consumer service experiences. However, the article is very light in terms of the method to be used in analysing a story. Also, their article posits narrative theory as an interesting new approach as part of a smorgasbord of interpretive methods but does not indicate any reasons why the method should be adopted at either an epistemological or ontological level.

\section{Story versus Narrative}

From the above it is clear that the distinction between story and narrative is unclear.

Often the terms are used interchangeably. According to Boje (2001), narrative is positioned as superior to story. Stories are based on actions or experiences undertaken by an individual or group of people about an event that can be real or imaginary (Boje et al, 1999). They can be seen as the special glue binding symbols, meanings and values, which are seen as the core attributes of culture (Myrsiades, 1987).

According to Myrsiades (1987) myths are a subset of stories. Myth and stories can be used to promote and reinforce dominant ideologies. In other words, stories are not value neutral (Boyce, 1996). Myrsiades (1987) makes the point that the roles of 
stories in an organization are both symbolic and also create order. 'More to the point, myths and stories function as communications of shared systems of meanings that allow organizations, as speech communities, to make sense of their environment' (Myrsiades, 1987 p. 90).

Narrative, on the other hand, is the retelling of a story by another person (Anderson, 1998, p. 169) and in doing so provides structure and interpretation of a story.

Therefore, a narrative approach to marketing management not only means collecting and interpreting stories but also applying the tools of literary analysis to those stories. However, before the structure of narratives is examined in more detail, it is important that the role of stories in an organisation is understood.

\section{Role of Stories}

Stories in the organisation can act as both substantive and processual symbols in the sense that they send messages to staff about acceptable actions and attitudes. Stories can be seen as a process of explaining. They provide answers to the question 'why?' They provide a basis for indicating the way 'business is done around here'. In other words, stories become a justification for norms and desired behaviour.

They play an important part in socialization of new members, generating commitment from staff, gaining influence of the values and norms within the organisation, and developing meaning and understanding by staff (Boyce, 1996). In a storytelling organisation collective stories are told, which involves a process of retelling and reshaping the stories so over time the insiders and outsiders get the story straight. Examples of stories in an organisation can be horror stories 
concerning the worst client in company history, or war stories based on winning an account from the competition. Whilst all organisations are storytelling it becomes a matter of where on the continuum they sit. At one extreme is a tightly controlled organisation where all stories are subordinated into the grand narrative, which then becomes the official company story. At the other extreme is the multiplicity of stories, storytellers and performances.

It needs to be remembered a story can involve an oral or written performance about a past event as well as an anticipated event. The event being told is an interpretation of the person's experience, past or future. As a story is a personal rendition of the experience it means that there is a plurivocity, in other words, multiple meanings can be voiced, which lends to participants being able to create their own interpretation of the experience. Therefore, the question then is to determine the criteria to judge a story. 'Each structural element of the story must stand up to scrutiny: subject, object, relationships between subject and object. Ultimately, the question is raised whether all of these together constitute a coherent and therefore field of action. By referring to the structure of subject, object, relationships, and field, the unfamiliar can become familiar because it can be assigned to structural categories already mutually understood between two subjects, each belonging to different worlds' (Hummel, 1991 p. 40).

Also, stories are used by people to make sense of their world, and in undertaking this task, discourse between members is central. As a means of motivation, getting to grips with a situation, and solving a marketing problem stories are used. Stories can 
take the form of recalled experiences, case studies, or purely descriptive studies, e.g. "When I worked in the fmcg business we solved the problem this way."

To motivate staff Hummel (1991) admits that there are many types of stories that managers can utilise but he outlines two main types, namely, the engaging anecdote and the biographical anecdote. The former invites the listener to become involved in the story by taking an active part, whereas the latter is about a recalled experience where the listeners are invited to broaden their own experience along with a new meaning.

People don't just tell stories for the sake of telling stories. Stories are used to 'enact' an account of either themselves or the community in which they belong, whether it is a work, recreational group, family or friends. However, from a researcher's point of view it is necessary to understand that there are different types of stories within an organisation, over and above the two mentioned by Hummel outlined above. One way to contrast stories in an organisation is to make a distinction between how the stories are obtained and who the stories are about.

Therefore, in situ stories are obtained through participant observation. Boje (1991) believes that stories obtained this way are more realistic as they take into account their natural performance context in terms of how the story was introduced, the reaction of the listeners and how subsequent dialogue is modified. In contrast to this there are stories that are elicited. In this case the researcher is asking the story-teller to be retrospective. 
In terms of whom the stories are about there are two general categories. The story may be about an individual in the organisation or it may be about the collective, which could be about the organisation as a whole or part of the organisation, for example, the sales team. From the above categories a matrix has been developed to describe types of stories (see Figure 8).

Figure $8 \quad$ Types of Stories

\begin{tabular}{l|c|c|}
\multicolumn{1}{c|}{ Individual } & \multicolumn{1}{c|}{ Collective } \\
\cline { 2 - 3 } In Situ & $\begin{array}{c}\text { Quadrant One } \\
\text { Gossip/chat }\end{array}$ & $\begin{array}{c}\text { Quadrant Two } \\
\text { Meetings/Discussion }\end{array}$ \\
\cline { 2 - 3 } Elicited & Quadrant Three \\
Reference & Quadrant Four \\
& $\begin{array}{c}\text { Grand narrative/ } \\
\text { Personal perspective }\end{array}$ \\
\cline { 2 - 3 } & \\
\hline
\end{tabular}

Therefore, in Quadrant One the stories tend to be centred around gossip, but in some cases it could be a directive from the manager. Such stories tend to take place informally, round the coffee machine, in the corridors or impromptu meetings around a person's desk. In Quadrant Two the stories are about the organisation and tend to take place in meetings and other formal get togethers. Often the stories that are told are used to make a point or indicate a desirable outcome. In Quadrant Three the story is elicited about a certain individual. It may take the form of a reference or it 
may be an attempt to ask for a justification why certain behaviour or action was undertaken. In Quadrant Four the stories are elicited from the organisation either as the official view, for example, a media release from corporate affairs, or a personal view of the organisation. 'There is an official discourse and there are many marginalised discourses in every organization' (Boje, 1995 p. 1009). As will be outlined in the next chapter, this thesis is interested in stories generated in Quadrant Four because managers interpret their world through story telling, which is a valid means for producing and accumulating knowledge.

However, as Boje et al (1999) note, research about stories can be perilous as researchers can be tempted to emphasise one version of a story over others and ignore some stories altogether. Stories gained in situ pose a problem in this regard as the best story may be overshadowed by the person who has the most power to make their story heard. Writing a narrative is always a matter of choice and selective requisition.

\section{Narrative Theory}

Stories are told firsthand whereas a narrative is the story told by another person. Whilst a story may ramble and provide a snapshot of an event, a narration involves a plot and a structure. For example, Joseph Conrad's (1986) 'Lord Jim' is an example of a story that is narrated by Marlow who puts the puzzle together with a structure and a plot.

Fisher (1985) developed a construct based on what he called a narrative paradigm.

However, it is more like a metaparadigm as it can be shared across various 
disciplines. Based on a notion of narrative rationality, Fisher believed that stories were based on two constructs. The first he termed probability, where the story is judged to the degree it hangs together and is free from contradiction. The second construct he termed fidelity, which questioned the logic of the story and its values. He saw that human communication functions as a way to make decisions and as a basis for action in constraining and determining behaviour. This is in contrast to the structuralists who were looking for meaning through linguistic constructions.

Whilst the above is helpful, it is necessary to explore narrative in greater detail by determining the essential features and how they are structured. In terms of their features, Pentland (1999) describes five essential elements. First, there needs to be a sequence in time, with a beginning, middle and end. Underlying narrative structures are stories or fabula. They reflect the deep structure of the narrative and are used to explain the surface structure. The event sequence is part of the fabula of a story. Second, the focus is upon either someone or something. There is often a protagonist, and frequently, an antagonist. The actors are part of the fabula and provide a thread to the story. Third, the narrative belongs to someone which means there is an identifiable voice with a specific point of view. 'Every story is told from a particular point of view, with a particular narrative voice, which is not regarded as part of the deep structure' (Pentland, 1999 p. 714). Four, narrative involve an evaluative frame of reference. Within the narrative there are implicit or explicit standards from which judgements can be made about the characters. Five, usually there is more than just the bare events. Such indicators are used to note time, place, attributes of characters. Whilst they do not advance the plot, they are significant in the interpretation of the 
event, e.g. whether the scene being narrated is that of an airline or an office supply company.

Characters are part of deep structure as they add cohesion to the story and a thread of continuity and meaning. The actors define the unit of analysis in a study. 'We cannot simply replace one role with another; because the identity of who performs an action can be a relevant part of a story (consider the difference between murder and suicide, for example)' (Pentland, 1999 p. 714).

When looking at the structure of a narrative the issue of credibility arises.

Sometimes a narrative goes beyond conventions where the story involves something incredulous, for example, the telling of a fantasy. Therefore credibility is not driven by facts but the forestructure of narrative conventions. According to Gergen (2001) there are six conventions to be upheld for a narrative to be intelligible.

The first is that a valued endpoint needs to be established. The event or action to be explained must have an outcome, which in itself is value ridden regarding good or bad, for example, narrowly escaped death. Therefore within the story there needs to be embedded an evaluative framework, explicit or implied, to judge the characters and actions. The second convention is that an endpoint dictates the events to be included. Obviously some accounts will make the events more vivid and/or more convincing thereby allowing the reader to suspend their disbelief. Therefore the narrative demands, in the sense of what gets included or left out, have ontological consequences with respect to what constitutes reality and what exists within that reality. 
The third convention concerns the ordering of events. Usually the story is told with a linear, temporal sequence but it is not necessary to tell the events in the order they occurred, as it will depend on the effect the narrator is trying to make, for example, in a murder mystery where events are revealed to put people off the scent. It also needs to be noted that the order of events may change over history as the narrative is retold.

The fourth convention is that the narrative must have a stability of identity. In other words, a character cannot be a villain then a hero in an unpredictable manner. Any changes in the identity of the character need to be explained. This can be done, for instance, by the use of war, famine or economic crises which have the effect of making the character change their ways.

The fifth convention is that some notion of explanation for the outcome is woven into the narrative tissue. This could loosely be called a causal linkage but this is not assuming a universal connection. Causal linkages assumed in a story are always dependent on a historical and cultural context. Finally, the narrative must have some demarcation lines to signal the beginning and end of the story. Therefore the story is framed by various rule governed devices, for example, the role of laughter or poignant pause. In conclusion, it can be seen that the use of conventions leads to coherence and direction in a narrative. 


\section{Postmodern Narrative Theory}

The above descriptions of narrative theory are based on a modernist view point and have been developed from the structuralists and formalists who were searching for regularities and underlying laws that were deemed to exist in the format of the story (Myrsiades, 1987). The Russian Formalists made a distinction between fabula, being the story, and sujet, representing the plot (Walsh, 2001). However, with postmodern narrative theory this distinction collapses as it is argued that one cannot master a story without reference to the plot. Any story involves a plot of some kind. The idea of fabula was an attempt to see narrative information as the bare facts, with an undistorted representation. However, fabula is linked with a point of view, and as such, is never innocent. 'Fabula is always relative to and contingent upon both a given sujet and a specific act of interpretation' (Walsh, 2001 p. 605). Therefore the distinction between fabula and sujet is rejected by the postmodernists as being based on a false dichotomy.

The question arises as to the difference between modern and postmodern narrative theory. Boje (2001) uses the term 'antenarrative' to describe postmodern narrative. What is important is that many voices are taken into account in a narrative in a fragmented, non-linear way. Antenarrative is never ending, whereas narrative attempts to ring-fence a story, making it coherent, sequential, and complete. 'Stories are antenarrative when told without the proper plot sequence and mediated coherence preferred in narrative theory. These are stories that are two unconstructed and fragmented to be captured by retrospective sensemaking' (Boje, 2001 p. 3). 
Therefore narrative and antenarrative can be combined so that a multi-voiced methodology is adopted. However, antenarrative is not to be confused with antinarrative, where the latter term is used to explain a story which is told in the present without plot or closure.

According to Boje (2001) there are five dimensions of antenarrative. The first involves speculation before a plot is determined and the outcome is imposed by narratology. The second draws attention to the ambiguity of sensemaking because narrative is retrospective. The third dimension draws attention to the flow of storytelling and admits to the plurivocality of stories where the focus is on the experience of the story-telling life. The fourth dimension involves the Tamara ${ }^{31}$ of storytelling regarding the flow of experience. '...the meaning of events depends on the locality, the prior sequence of stories and the transformation of characters in the wandering discourses' (Boje, 2001 p. 4). Finally, antenarrative is before the story is reified into an agreed plot, therefore the narrative is still in a state of flux. Postmodernism makes coherence problematic, due especially to the notions of fragmentation and simulation. The researcher can only obtain bits and pieces in an attempt to work out the puzzle but is unable to complete the whole picture, therefore finding coherence is difficult to obtain, unlike a modernist account which demands coherence.

A postmodern narrative is different from a case study where someone else's story is narrated and an attempt is made to make sense of it, usually with a moral or business

\footnotetext{
31 Tamara Land, mentioned by Boje (1995) with reference to his analysis of Disneyland, is a long running play in Los Angeles where the play is staged in many rooms and the audience gets to follow an actor from room to room. Depending on who you follow will determine the story you will hear. 'Instead of remaining stationary, viewing a single stage, the audience fragments into small groups that chase characters from one room to the next, from one floor to the next, even going into bedrooms, kitchens, and other chambers to chase and co-create the stories that interest them the most. If there are a dozen stages and a dozen storytellers, the number of story lines an audience could trace as it chases the wandering discourses of Tamara is 12 factorial $(479,001,600)^{\prime}$ Boje, 1995 p. 998).
} 
principle being evoked. Many business case studies, for example, the Harvard case series, take this position and are elitist in nature (Boje, 2001), in the sense that the case is written from the position of the CEO, who is portrayed as being the saviour or hero of the organisation.

\section{Narrative Analysis}

When it comes to narrative analysis Richardson (2000) believes that there are four basic types of narrative method. The first looks at the sequence of events as a defining feature, the second looks for causal links, the third takes the view that any statement of an action or event is by definition a form of narrative, and the fourth believes that narrative is simply a way of reading a text. The first two types tend to represent the majority of narrative analysis. However, postmodern narrative analysis takes on a new form. Boje (2001) in 'Narrative Methods for Organizational and Communication Research' outlines eight methods for conducting narrative research, namely deconstruction, grand narrative analysis, microstoria, story network analysis, intertextuality, causality analysis, plot analysis and theme analysis. It is apposite that a brief description is outlined of the first seven methods, with the last method, theme analysis, explored in more detail as it is an appropriate method to use when attempting to understand praxis, as it utilises all the other narrative methods, such as plot analysis and microstoria.

According to Boje (2001) the essence of deconstruction is antenarrative. Based on the work of Derrida (refer to Chapter Five) it is argued that deconstruction is not formula-driven where certain steps and procedures are vigorously followed. The main aim of deconstruction is to decentre authoritative narratives as no story is 
ideologically neutral due to it being imbued with social and political values. This does not mean that one voice should be replaced with another, for example, from masculine to feminine, but to show how different voices change and ultimately disintegrate a story, in the sense that the story will lack cohesion.

Despite Derrida's stance regarding a strict method, Boje (2001) outlines eight steps to conduct deconstruction analysis. Briefly, these are as follows. First, search for any words that have a dichotomous meaning within the story. Second, read the story to determine the hierarchy that is implied. Third, look for other voices not represented in the story. Fourth, search for the other side of the story. For example you may want to look at the union's voice compared to management's voice. Fifth, change the plot. In other words, if it is a comedy then change the story to a satire. Sixth, look for exceptions in the story that make the story absurd. Seventh, look between the lines to determine what has not been said. In other words, fill in the blanks that the narrator assumes the reader knows. Finally, rewrite or re-author the story beyond any one voice or singular point of view so that a new balance of views are represented.

The final step is the most important as it removes domination and hierarchy contained in the original story. However, as each story reaches stage eight the process can begin again so that the new resituated story can be deconstructed, and so on and so forth. The main aim of the process is not to deconstruct a story for its own sake but to look at a story from different perspectives so as to gain a more insightful understanding of an event. 
The second type of narrative analysis outlined by Boje (2001) is that of Grand Narrative, where the story is based on a macro view of the event. Such stories are told at the expense of other minor stories that resist the main thrust of the plot. A grand narrative can be seen as a metanarrative that contains a system of truth and by taking a dominant position it marginalises other discourses. Boje (2001), using Lyotard's original typology, outlines ten grand narratives (refer Table 3). Note that postmodernism has been added as a grand narrative but not all grand narratives should be seen in a negative light as they can be resituated to show different beneficial aspects. However, the main point is that grand narratives take a privileged position and from that hegemony develops where one voice dominates.

\section{Table $3 \quad$ Ten Grand Narratives of Enlightenment Knowledge}

1. Logical positivism

2. Imperial politics

3. German idealism

4. Critical enlightenment

5. Self-management

6. Marxism

7. Frankfurt School

8. Cybernetic systems theory

9. Post-industrial capitalism

10. Postmodern condition

Source: Boje 2001 
Grand narrative can be based on three major constructs, namely essentialism, universalism and foundationalism. Some grand narratives appeal to the essential nature or essence of an idea. Where universalism is involved the objective is to find laws or principles which leads to totalising truths, where narrative differences are glossed over. The effect of this is to lead to the one and only story being told, for example, the American Dream based on the principle that everybody has the same opportunity to realise their own wealth. Finally, foundationalism is the search for the foundation of knowledge, based on the idea that there is only one truth. For example, Kant's quest for the basic components of moral reasoning.

The important point is to be aware of the underlying constructs of the grand narratives but this does not necessarily mean that they must be rejected. Instead the task is to resituate them and to re-story them so that minority voices are heard and new perspectives are obtained.

Another approach to narrative analysis is that of microstoria, which is interested in stories of minority groups or the little people. By its focus it tends to be antenarrative and researchers in this area are very sceptical of grand narratives and the enlightenment project. For them they are interested in local ways of knowing so that there are multi-centred rather than one official voice. Their approach is to use archival evidence obtained from secondary data and reconstruct a situation about everyday life.

In contrast to deconstruction, microstorians believe that history deals with real subject matter which is outside the text, existing in social and natural reality. 
Therefore there is a belief that there is such a thing as the right reading.

Microstorians want to limit their research to a real time and place. 'In sum, the ontological approach of microstoria is one in which there is knowledge that is specific to time and place, that can be read in the material remains of stories recorded in various archives and diaries. What is antenarrative is the focus on entering the webs of storied relationships and meanings, the stories and counter-stories of that time' (Boje, 2001 p. 49).

Unfortunately microstoria needs a macro social context to give the local stories meaning and without this they can be charged with methodological irrationalism and trivialisation. On the other hand, microstoria presents another view counter to the mainstream elite stories centred on CEO's, managers and owners of an organisation, at the expense of secretaries, factory workers and cleaners.

Another method outlined by Boje (2001) is that of Story Network Analysis where the task is to organise and categorise stories into narrative maps, using nodes to represent people and lines to represent the relationships that exist between people. What is of interest from an antenarrative perspective is the living relationships that link the people. However, basically this is a structuralist approach to story. 'Human processes of meaning construction and embedded social historical dynamics in the construction and transformation of networks can become reified as "object analysis" in structuralist analysis. Reification is forgetting that a structural object-relation display has subjective meaning and social construction processes beyond the map in the context' (Boje 2001 p. 64.) 
The fifth method of analysis outlined by Boje (2001) is that of Intertextuality where the task is to look at the links within a story which means that there is no homogeneous story but a patchwork of small different stories, dependent on each other. In organisations, especially within marketing departments, new texts are added everyday and each text is inter-related. Therefore, a story is not a closed narrative which can easily be compared with other texts. Instead, stories can be understood in two ways. First is by what Boje calls 'the heterogenous stitch and weave of utterances of a text' (Boje, 2001 p. 74). The second is by seeing the text as an ongoing dynamic network of production, distribution and consumption.

The metaphor that best describes this type of analysis is that of the carnival. This can be exemplified by two streams regarding the production of texts. The first is horizontal where there is a chain of events which take place in a linear fashion. Hyperlinks from one text to another are made by way of references, footnotes, etc. The second stream is vertical which has non-linear time scales and links are made by way of contempories, genres, discourses and styles. 'The methodological task is to unravel a text's intertextual network of attributed and unattributed links to other texts' (Boje, 2001 p. 92).

In general narratives attempt to answer the question why? The task is to look for plausible explanations by identifying a cause and effect. But as Nietzsche asks, which comes first? Is it the effect so one looks for a cause or is it the other way round? Therefore, causality is an invention based on a fiction and linear causation is a convenient fiction. In standard narrative analysis a content analysis is undertaken to identify causal assertions which can then be mapped. However, searching for 
causal links is not easy. 'The causal field is messy and often unfathomable and acts of narration camouflage that antenarrative fabric of complex organizations' (Boje, 2001 p. 107).

It is further complicated by multiple interpretations of an event. However, as the emphasis is on the construction of meaning there can be no best explanation. In line with an anti-foundational approach the idea of a correct explanation does not make sense. Therefore attempting to find a strict logical cause and effect is pointless. At best with causal analysis, it is possible to sketch some tentative explanations to the question why.

The most common method of narrative theory is that of plot analysis. A plot is the structure that binds the story together. A plot therefore is a structure of events. However, whilst a narrative has a plot, it is not intrinsic to the event but is imposed by the author (Brown, 1998).

According to Currie (1998), plot analysis comes from Aristotle who developed the classic plot typology of comedy, tragedy, romantic and ironic or satirical. With comedy the plot is about the triumph over the dark forces of evil with the final outcome being harmony between conflicting parties. It is possible to get a combination of plots such as a romantic/comedy. An example of this is the film 'As Good As It Gets' where the relationship between Melvin Udall, played by Jack Nicholson, and Carol Connelly, played by Helen Hunt, sours and in fact goes downhill markedly then improves towards the end of the movie. Tragedy, on the other hand, is where the hero is defeated by experiences that he endures, but the 
audience is left with hope that liberation, the eternal dream, is possible. With romance the heroine gains ascendancy over her miserable existence and the hero is redeemed and hence liberated by achieving a quest, for example, slaying a dragon. 'Pride and Prejudice' would be a good example of a romantic plot. Finally, the satire is the opposite of romance where the heroine is captive in her world and is surrounded by darkness or a void with little hope of harmony. Whilst it can be argued that all types of plot have a moral overtone, it is the satire that is used poignantly to make explicit man's folly, hubris and immortality.

A narrative plot can be seen as a series of actions which give the rationale and expectations for those actions. Narrative theory stresses the role of language in discourse and our everyday encounter with the world. According to Gergen (2001) there are three rudimentary forms of narrative, with the first being a stability narrative where the trajectory of the story essentially remains unchanged in relation to the outcome. The second form is that of a progressive narrative where the movement is along an evaluative dimension with the outcome being positive or successful. The third form is that of a regressive narrative where the movement is decremental in the sense that the end of the narrative is worse than when it started.

What needs to be determined with plot analysis is the links that hold events in a story together, so it is more than just a chronology of those events. This means that the structure of the plot is important. From an antenarrative point of view the issue is who controls the plot and how they are presented in a social system implied by the story. 
Theme analysis is the final method outlined by Boje (2001). It is the most important method as it brings together all the other methods that have been outlined. The task of theme analysis is to categorise a story into different themes. There are two primary ways that this can be achieved. The first is to use a deductive approach whereby the themes are applied from the outside. In other words, the themes are imposed upon the story from a theoretical structure. For example, the marketing mix can be imposed upon a case to determine how a given marketing strategy was implemented. Such an approach according to Boje (2001) is termed an etic taxonomy. The second way to categorise a story is using an inductive approach whereby the themes of the story emerge from the transcripts of the interviewed. The term emic is used to refer to the way the storyteller sorts their story as they tell it. Therefore the themes arise from inside the story. However, it needs to be noted that these are not mutually exclusive methods as both approaches can be used to examine a story.

Basically the task of theme analysis is to search for patterns. These patterns can then be mapped on to a paradigm worksheet as outlined below (see Figure 9). The purpose of the chart is to capture complex set of themes and counter-themes. The objective is to contrast the themes of a narrative rather than accept the one-sided dichotomy presented in the narrative. The first contrast is between a monophonic voice, for example, the company spiel which is single-voiced and contains a high level of coherence, and polyphonic voices, for example, the views of different participants within the company which may be at odds or show some variance to the single voice. The second contrast is between the types of knowledge utilised within the story. It may be scientific knowledge evidenced by rational discourse based on 
theoretical constructs, or it may be aesthetic knowledge evidenced by creative

discourse based more on hunches or intuition. As will be noted, the above contrasts lead to four distinctive themes that can be analysed, with antenarrative as a fifth cell linking them together.

\section{Figure 9 Basic Narrative Themes}

\begin{tabular}{|c|c|c|}
\hline $\begin{array}{l}\text { Key } \\
\text { - Etic } \\
\text { - Emic } \\
\text { Both } \\
\text { Antenarrative }\end{array}$ & \multicolumn{2}{|l|}{$\begin{array}{l}\text { Monophonic } \\
\text { Polyphonic } \\
\text { narrative } \\
\text { narrative }\end{array}$} \\
\hline \multirow{3}{*}{ 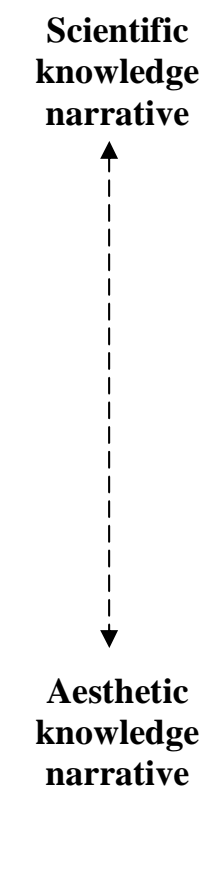 } & $\begin{array}{l}\text { BUREAUCRATIC } \\
\text { - Hierarchy } \\
\text { - Red tape } \\
\text { - Functional } \\
\text { - Stuck in tradition }\end{array}$ & $\begin{array}{l}\text { CHAOS } \\
\text { * Chaos (emic/etic) } \\
\text { - Complexity } \\
\text { Adaptive systems } \\
\text { Edge of chaos }\end{array}$ \\
\hline & \multicolumn{2}{|c|}{$\begin{array}{l}\quad \text { ANTENARRATIVE } \\
>\text { Between the boxes } \\
>\text { Flows between cells } \\
>\text { Intertextual } \\
>\text { Polysemous } \\
>\text { Multi-layered and embedded } \\
>\text { Story networking behaviours } \\
>\text { Excess not in this taxonomy }\end{array}$} \\
\hline & $\begin{array}{l}\text { QUEST } \\
\text { - Call (individual/organization) } \\
\text { - Journey (individual/organization) } \\
\text { - Return (individual/organization) } \\
\text { * Reorganization adventures }\end{array}$ & $\begin{array}{l}\text { POSTMODERN } \\
\text { - Post-industrial } \\
\text { - Post-Fordist } \\
\text { - Postmodern }\end{array}$ \\
\hline
\end{tabular}

Source: Boje 2001 p. 124

Themes that will display qualities of the bureaucratic typology will have discourse that emphasises functionality, division of labour, red tape and bounded rationality 
with reference to theoretical constructs. Such narratives are optimistic, holding hope for the organisation with progress being the benchmark evidenced through talk of Porter's five forces, SWOT analysis, Total Quality Management, Service Quality or the implementation of a Balanced Scorecard. However, it needs to be acknowledged that bureaucratic organisations also have a dysfunctional side to them, as noted by Weber (1930) when he talks about the iron cage of rationality, and also by Ritzer (2000) where he discusses the 'irrationality of rationality'.

The chaos narrative displays chaos and complexity and is in contrast to the bureaucratic typology. Such a narrative will talk about the organisation being dynamic and having a very lose structure. There will be evidence that the organisation is out of control and disorder prevails. This is in contrast to the mechanistic systems of the bureaucratic type organisation.

Quest narrative is similar to a plot analysis but follows a standard drama type model where the hero embarks on a journey of discovery, goes through many trials and tribulations and in doing so returns triumphant transformed personally or his social milieu is transformed, usually for the better. Such narratives are single-voiced but with many characters. This is a popular culture type of category and is used in movies and television dramas, for example Star Wars and Lord of the Rings. Stories about organisations often follow this format and are usually based on a strong CEO who has led the organisation from a loss situation to become a profitable enterprise. Herb Kelleher, the CEO of Southwest Airlines has many narratives written about his success based on this format. 
A postmodern narrative is one where there are many voices telling fragmented pieces of the story without referring to the grand narrative. Such narratives would be localised without looking for any generalisations and truths. From a research point of view, it is possible that a respondent would refer to the four themes when telling their story. In fact the drama of the story would be enhanced by the conflict which arises from the juxtaposing themes.

In the middle of the diagram is antenarrative which is the link between the four cells. Often this constitutes what is not said, that is, what has been left out of the narrative. Such an analysis would give additional insights over and above the themes contained in the four cells.

This section has outlined eight ways to analyse narratives based on a postmodern perspective. From the point of view of this thesis, the favoured approach is that of theme analysis as it builds on the first seven approaches and allows for the stories of marketing managers to be explored using etic and emic frameworks. In addition to theme analysis, marketing managers' narratives can also be examined in terms of sensemaking. This will now be explored.

\section{Sensemaking in Organisations}

According to Boje (1991) storytelling is the preferred method of sensemaking in organisations. Over time the stories get refined and polished. Also the story is modified according to the audience. For example, whilst the executive team hears one version of the story (the team member was dysfunctional due to problems at home so she was asked to reconsider her position), the line staff will hear a modified 
version (she was underperforming) whereas the external stakeholders will only hear a snippet (she got fired). Such stories serve as precedents for decision-making and justifying actions and serve as the main stay of institutional memory for the organisation. Therefore, everyone has their own story, modified, moulded and adopted over time to suit different purposes, that is, the need of the audience. 'Both teller and listener are sending cues to manage how much of the story is told, how much is left to the imagination, and what interpretation is applied. This also is management of sense making' (Boje, 1991 p. 124).

Sensemaking can be used within organisations as a means to manage the shared beliefs of employees. For example, Allbright (1999) argues that sensemaking can be used as a competitive advantage, alongside the logic of planning and control, and that of task pursuit.

Over and above stories being used as a management technique to manage the organisational culture, they are utilised by individuals to make sense of their world, whether it be work, leisure or personal activities and also utilised in an informal, collective sense, where stories are shared as group members to either make sense of the past or put future actions into a perspective. Narrative approaches '..illuminate how multiple, ongoing narratives contribute to sense making and to its outcomes: decisions and actions' (O'Connor, 1997 p. 397).

People encode problems into narratives, which allow them to put these problems into perspective and make sense of them. Often people do not initially make sense of a problem so they will retell the story in an effort to put the issue at rest. You will hear 
phrases like, "I cannot understand why she did this!" or "It doesn't make sense to me. You think she would have..." Such phrases are used in a story as a means to make sense and gain an understanding.

Sensemaking, according to Weick (1995), is mainly due to the ability of agents to think in narrative terms. The ability to look back retrospectively allows a person to construct an understanding of events and consequently give it meaning. This involves the person invoking explicit and implicit mental processes to be able to construct and frame so as to have a perspective of an event or situation. 'To talk about sensemaking is to talk about reality as an ongoing accomplishment that takes form when people make retrospective sense of the situations in which they find themselves and their creations. There is a strong reflexive quality to this process' (Weick, 1995 p. 15).

According to Weick (1995) there are seven distinguishing factors to sensemaking. The first is related to identity-making. The second factor is retrospection as it is believed that knowledge of an action comes after it has been undertaken. The third factor involves cognitive bracketing. The fourth factor is to acknowledge that sensemaking is social as the conduct of others impinges on a person's story. Another factor is that sensemaking is ongoing over a period of time. Stories are revisited and reshaped to fit either new information or new contexts. The sixth factor is that sensemaking is extracted by cues. The final factor is that plausibility is more important than accuracy.

'If accuracy is nice but not necessary for sensemaking, then what is necessary? The answer is, something that preserves plausibility and 
coherence, something that is reasonable and memorable, something that embodies past experience and expectations, something which resonates with other people, something that can be constructed retrospectively but also can be used prospectively, something that captures both feeling and thought, something that allows for embellishment to fit current oddities, something that is fun to contrast. In short, what is necessary in sensemaking is a good story' (Weick, 1995 p. 60).

Magala (1997) has made a number of criticisms about sensemaking. First, he asks what constitutes sense. It is not clear that Weick has articulated a clear construct for this concept. Second, Weick does not describe how sensemaking operates. He takes a constructivist view of sensemaking, based on cognitive psychology, but does not articulate a psychological foundation. Finally, Magdala questions the position of emotions in Weick's framework.

Notwithstanding the above criticisms, sensemaking does provide a rich context for decision analysis and how executives give meaning to their situation. Magala (1997) also notes that sensemaking repositions the role of the agent within organisational decision making. As noted by Craig Lees, 'Knowledge about the mechanics of thinking, information processing, problem-solving techniques, and memory processes is substantial. Knowledge about the factors that influence how individuals in organizations interpret data, set out reasoning and evaluation, and use stored knowledge to select and shape incoming information is limited (Craig-Lees, $2001 \mathrm{p}$. $515)$.

However, as mentioned above, Weick bases his view of sensemaking on ac cognitive psychology (Craig-Lees, 2001; Hopkinson, 2001). Such a position sees a story as a realistic account of an event. This explanation sees a simple relationship between language and meaning. 'The objective in cognitivist research is to identify and reproduce the sense-making frames of the researched community or to discover how 
their system of categorization operates (Hopkinson, 2001 p. 425). Following on from this, narrative is seen as a representation of cognitive reality.

In contrast to the above approach to sensemaking is that based on social constructionism, where sensemaking is seen as a discursive practice, providing understanding of the world. On this basis reality is situated, fragile and negotiated. 'Sense is therefore dialogic or negotiated at both the individual and at the organizational levels' (Hopkinson, 2001 p. 425). In other words, reality is communicated through language.

This is in contrast to the dominant logic in marketing management has been to conduct single person, one time-only surveys to collect data (Woodside, 2001). Unfortunately this method gains information from only one person so the data gained tends to be shallow and based on inaccurate views. Data gathered by looking at the sensemaking of marketing personnel, based on a narrative theory methodology allows for the understanding of praxis within an organisation.

As has been outlined in Chapter Three, empiricism sees behaviour as objective and verifiable, and as such, exists independently of a participant's understanding. Sensemaking challenges this view. From the point of view social constructionism, sensemaking is a discursive practice, negotiated at both the individual and the organisational level. Positioning is an important concept in sensemaking as it is linked to the social embeddedness of discursive identity as an individual articulates a story in relation to others and also in comparison to others (Hopkinson, 2001). 
Therefore the author of the narrative is important because such a person selects certain relevant (to them) elements and brings them together in a plot.

As sensemaking is discursive it allows a researcher to understand how marketing actions were undertaken. In other words, sensemaking gives a justification why past marketing management decisions were implemented. Conversely, sensemaking allows a researcher to understand how future marketing decisions are likely to be made.

Whilst this thesis adopts social constructionism's view of sensemaking, in line with the linguistic turn in philosophy, certain distinctions need to be made regarding stories and sensemaking. First, one of the criticisms of story methods relying on interviews is that they ignore performance behaviour (Boje, 1991). Such interviews do not relate the actual behaviour at the time of the participants as they went about their business on a day-to-day basis. In other words, whilst they may be of interest and serve a purpose, such research does not convey what was happening in the natural context of the situation. Boje suggests that stories told in an interview are different from stories told on a day-to-day basis to reinforce behaviour, set goals, and make a point of why one action is better than another. However, stories told of past events is still a legitimate way to gather information.

Another important point is that there is a symbiotic relationship between narrative theory and sensemaking. On the one hand, sensemaking uses narrative theory, via stories, to understand how people make sense of their situation. On the other hand, sensemaking is used as a mechanism to elicit stories so that narrative theory can be 
applied, by a number of methods as outlined in this chapter. This thesis will utilise the second sense, where sensemaking is used as a cue to elicit stories s o that an understanding of praxis can be obtained. As has been explored in this section, everybody utilises stories to make sense of the problems and issues they face on an everyday basis, so they are readily available as a means to understand praxis. It is this application that will be explored in the next chapter.

\section{Conclusion}

The purpose of this chapter was to explore narrative theory as a method to build a new perspective of marketing management knowledge based on the linguistic turn in modern philosophy. Stories as a means of obtaining knowledge was outlined and a matrix developed to distinguish different types of stories. The difference between stories and narrative theory was examined, followed by a consideration of the structure and features of narrative theory. Postmodern narrative theory was then explored. Eight types of postmodern narrative theory analysis were explained and it was deemed that theme analysis could be usefully employed as a way to generate marketing management knowledge. This analysis can be further enhanced by linking narrative theory with sensemaking in organisations.

The next chapter will utilise the paradigm framework outlined in Figure 9 to explore a marketing management decision, namely the domestic Express class launched by Air New Zealand. A grand narrative will be developed from information gained through secondary sources. Juxtaposed to this will be the narratives of three key decision makers involved in the launch. From this different themes will be analysed and it will be shown how praxis takes place in an organisational setting. 


\section{Chapter 7: Exemplary Case Study - Air New Zealand Domestic Express}

\section{Introduction}

The thesis argues for the marketing management discipline to adopt the linguistic turn in modern philosophy, and in particular, adopt neo-pragmatism as a research programme. This would mean that the discipline rejects Cartesian dualism, and consequently adopts an anti-foundational stance as well as rejecting essentialism. In its place is edifying philosophy based on the ontological belief that human beings are dialogical, concerned with the development of understanding. This leads to the concept of praxis and how it can be understood in an organisational setting. Chapter Six outlined narrative theory as a means to explore praxis. Narrative theory was adopted as a methodology because it is an appropriate way to understand practical thinking, allows for multiple voices to be heard, overcomes the privileged position of the scientific approach to knowledge generation, and allows for hermeneutics to be used as the ontological base for the methodology. Therefore, the task of this chapter is to apply narrative theory as a methodology to an organisational strategic marketing problem.

\section{Objective}

The main aim of the case study is to exemplify a neo-pragmatism research protocol as the thesis has argued for the marketing management school to adopt the linguistic turn in philosophy. In turn, it has been argued that neo-pragmatism should be utilised as a research programme. As noted above, the task is to develop edifying philosophy, with the central theme being that of praxis, that is, practical judgement 
linked with action. Therefore, the research question that needs to be addressed is as

follows: How can the concept of praxis, that is, judgement and action, be understood in the context of a strategic marketing decision.

\section{Background Literature}

The purpose of this section is to give a background to the literature concerning judgement, keeping in mind the research question is to explore praxis, that is, the interaction of judgement and action, in the context of a strategic marketing problem.

Within marketing there has been a great deal of literature concerning decision making (Wilson and Woodside , 2001) based on different information processing models. Also, there has been papers based on decision support systems. For example, Lilien, Rangaswamy and Matanovich (1998), show how marketing engineering, based on spreadsheet decision models, would help the consistency of decisions and facilitate the impact of different variables. Another example is that of Bruggen, Smidts and Wirenga (1998), who claim that marketers are being confronted with an increasing amount of information so would increase their effectiveness of marketing decision making by utilising a marketing decision support system (MDDS). However, they state that the marketers who would benefit the most from a MDSS are low analytical decision makers and also those operating under low time pressure.

Alongside the issue of decision making is that of marketing planning, because often marketing decisions are made within the context of the marketing plan (Greenley, Hooley and Saunders, 2002). Kotler claims that the marketing plan is central to the 
marketing activity and is important for the co-ordination of activities. Such plans are based on a notion of rationality which decisions need to adhere to. As Ardley (2006) notes, marketing decision making takes a prescriptive approach and is based on a rational technical premise. Brownlie \& Spender (1995) argue that marketing plans, where detailed plans are developed and measured, are not reflected in practice. Part of the problem is that marketing managers have to deal with a lot of ambiguity and uncertainty in the decision making. Also, As noted by Murray, O'Driscoll and Torres (2002), a prescriptive literature prevails in marketing with a universal view where one-size fits all.

Following on from the above, another area within the general area of decision making is that of managerial competencies. Gilmore (1997) notes that marketing management competencies are related to their level of decision making. Along the same theme, Rose, Moore and Staelin (2000) explore the issue of marketing decision making on the effects on firm performance, taking into account the decision's complexity.

Whilst the above themes are central to how decisions are made within an organisational context, the question arises as to how individuals make decisions. One approach is that presented by by Wierenga and van Bruggen (1997), who use a cognitive approach to explore the decision making processes of managers. They argue that there are four types of distinct activities involved in marketing decision making. The first is optimizing where the managers searches for the best outcome. The second is that of reasoning, where the manager uses a mental model to understand the problem. The third activity involves the manager analogizing by 
searching for similar problems and outcomes in the past to make sense of the present problem. The manager may delve into their own experiences but also utilise cases that have arisen from either companies operating in the same category or other industries. The fourth activity is creating, which involves the manager developing novel and effective ideas to solve the problem. The first two activities are termed analytical and the last two activities are termed associative.

However, the four modes are not mutually exclusive in the sense that only one activity can be used to solve a problem. In other words, a marketing decision maker can use different modes to solve the one problem. However, Wierenga and van Bruggen believe that there is a dominant mode based on what the decision maker uses the most. Also, it is important to note that the activities are not stages that a decision maker goes through, unlike Piaget's theory of cognitive development or Kohlberg's model of moral reasoning, which are both based on a cognitive psychology.

Wierenga and van Bruggen note that the utilisation of the above modes will be hampered by the characteristics of the decision maker and the context of the decision making, where time constraints, market dynamics, and organisational culture will influence the decision making process.

The authors note that there has been very little empirical work undertaken in the way marketing managers make decisions in different markets and under different circumstances. 'It is surprising to see, in a field in which so much is known about consumer decision making, how little research has been done on how marketing 
managers actually do make decisions (Wierenga and van Bruggen, 1997 p. 34

Emphasis in original).

However, the above discussion of the literature has focused on marketing managers' decision making, and has not been about judgement. The cognitive model does not explicitly discuss the concept of judgement it can be seen that the associative types, that is, analogizing and creativity, would involve judgement. Nonetheless, decision making is not the same as judgement. As discussed in Chapter Three, judgement is an independent variable and differs from decision making based on analysis as it comes into play when the decision maker is faced with uncertainty in the situation being confronted. From a postmodern perspective, an ambiguous situation is socially constructed. 'Ambiguity does not exist somewhere in the world out there; it is the constant companion of social actors (marketing managers in this case) in their attempts to makes sense of the world' (Brownlie and Spender, 1995 p. 41). Therefore, judgement comes into play when there is insufficient information, due to either incomplete analysis or too much complexity, created by competing demands. This can be summed up by the general term 'uncertainty'.

According to Brownlie and Spender (1995) there are four types uncertainty. The first is where uncertainty is caused by incompleteness of information, meaning that one is ignorant of all the details. The second type of uncertainty is caused by indeterminacy, with respect that the interests of other actors in the organisation need to be taken into account. The third is where uncertainty is caused by a dissonance between theoretical models and the situation which is being confronted, resulting in the model being disregarded. Finally, uncertainty is caused by incommensurability, 
that is, knowledge of the situation has not been integrated into a conceptual model or framework, so the decision maker has trouble making sense of the situation. 'So, in the context of strategic decision making, the exercising of judgement is a collective activity that is informed by, and derives legitimacy from, the judgements of individuals and groups' (Brownlie and Spender, 1995 p. 412). However, Brownlie and Spender, (1995) believe that analysis and judgement support each other.

However, it is when there is ambiguity and the decision is complex judgement comes into play.

To explore the issue of judgement in more detail, Brownlie and Spender (1995) utilise a Strategic Balance Matrix (see Figure 10).

Figure 10 Strategic Balance Matrix

\begin{tabular}{l|c|c|}
\multicolumn{1}{c}{} & $\begin{array}{c}\text { Analysis } \\
\text { (perfections and } \\
\text { certainties) }\end{array}$ & \multicolumn{1}{c}{$\begin{array}{c}\text { Judgement } \\
\text { (imperfections and } \\
\text { uncertainties) }\end{array}$} \\
\cline { 2 - 3 } $\begin{array}{c}\text { Resources } \\
\text { (our costs) }\end{array}$ & B & A \\
\cline { 2 - 2 } $\begin{array}{c}\text { Markets } \\
\text { (other's values }\end{array}$ & C & \\
\cline { 2 - 3 } & & \\
& & \\
& & \\
& & \\
\end{tabular}

Source: Brownlie and Spender, 1995 p. 46 
The vertical dimension of the matrix distinguishes knowledge of assets from knowledge of opportunities. Knowledge of assets refers to a firm's resources within the organisation, whereas knowledge of opportunities refers to the market environment outside the organisation. The horizontal axis, on the other hand, refers to areas of high uncertainty in contrast with areas of low uncertainty. As can be seen it is in the area of high uncertainty where judgement comes to the fore. This is in line with the comments made in Chapter Three where it was stated that when information is not forthcoming, judgement is used as a fall back position, but it needs to be acknowledge that judgement is not a substitute but an independent variable.

Following on from this, good judgement cannot be assumed.

'Each cell implies the application of different kinds of managerial skills: cell A is the province of the creative and visionary; cell $\mathrm{B}$ of the bureaucrat, able to devote careful attention to detail and organization; cell $\mathrm{C}$ is similar to cell $\mathrm{B}$, but it involves affairs outside the firm and is the traditional domain of the salesman and marketer; and cell D is the province of the negotiator who can deal comfortably with those who have power over the firm's affairs' (Brownlie and Spender, 1995 p. 46)

In a large organisation there could be a role for each of the quadrants and the task of the leader is to create a cohesive culture which acknowledges each person's contribution to the task at hand.

In summary, the degree of judgement will vary depending on the informational context and the complexity of the situation. Whilst the above discussion implies the role of human agency, what is missing is the part action plays in the development of praxis. Therefore, the task of the exemplary case study is to explore how judgement and actions are carried out by participants in an organisation when confronted with a complex problem. 


\section{Methodology}

In undertaking this case study, the following method was utilised. First a grand narrative was written on the introduction of the Air New Zealand domestic express service based on information gained from secondary sources, namely newspapers and business magazines. The next step was to analyse the grand narrative in terms of the underlying constructs. Three interviews were conducted with personnel from the organisation who were involved in the decision and implementation of the strategy. Plot summaries were then developed and a plot analysis of their stories was undertaken. This was followed by theme analysis of their stories. Finally the stories were mapped on a grid using an adaptation of Boje's narrative theme framework, as described in the preceding chapter.

The criteria for selecting a suitable company to use as a case study were determined by a number of factors. First, the company had to have a problem that needed a number of people that were involved in the decision making and implementation of the marketing strategy. This was necessary so that different perspectives could be gained from the participants.

The second factor that needed to be taken into account was the need of the problem to have marketing implications. It was not so much that the problem per se needed to be a marketing issue but more that the ramifications involved marketing resolution. In other words, marketing management decisions needed to be made by the company. 
The third factor was that information about the problem was available in the public arena, so that an objective grand narrative could be developed. On a day to day basis there are a number of marketing decisions that companies make. However, most of them are not made available through secondary sources such as newspapers and business magazines.

To meet the above criteria, Air New Zealand was selected as an organisation and the particular issue investigated was the launch of their domestic express service, in November 2002.

Initial contact was made with a senior executive in the organisation and three people were identified as having key input into the launch of the new service. The three participants were invited to participate in the research and a letter was sent outlining the background to the interview (see Appendix A). A prompt sheet was developed to assist in the interview (see Appendix B).

The participants were given the following verbal instruction by the interviewer: "I have written a realist account of the introduction of Express Class in the domestic market and framed it in terms of the marketing mix. The information for this case was gained from secondary sources, e.g. newspaper and magazine articles.

In contrast to this I am interested in your story ... your perspective on the issues surrounding the development of the strategy and the implementation of domestic Express. Casting your mind back, how did you make sense of such an important change in direction for the airline?"32

\footnotetext{
${ }^{32}$ It is important that certain ethical issues are acknowledged in this research. A research proposal was submitted to the Victoria University Ethics Committee for approval, which was duly granted.
} 
A broad question was used as Chase notes, 'It may be easy to articulate a broad open question that will invite a personal narrative' (Chase, 2005 p. 662). However, such an approach means that the resulting story cannot be known, predicted or prepared in advance.

According to Chase (2005), the interviewer can adopt a number of voices. The first is that of an authoritative voice, where the interviewer asserts their voice on the story, as they often have a different interest from that of the storyteller. In other words, they have their own agenda. This type of voice is open to criticism, according to Chase (2005), as the interviewer's stance is at the expense of the storyteller. The second type of voice is supportive, where the story-teller's voice is pushed into the limelight. The researcher makes decisions at the end of the interview process which part of the story to include but do not, according to Chase (2005), dwell on the interpretation. 'At its best, however, this narrative strategy aims not for establishing authenticity but rather for creating a self-reflective and respectful distance between researcher's and narrator's voices' (Chase, 2005 p. 665). The supportive voice was adopted in this case because it was important to hear the different voices to gain a multi-voiced perspective. 'Furthermore, when researchers treat narration as actively creative and the narrator's voice as particular, they move away from questions about the factual nature of the narrator's statements. Instead, they highlight the versions of self, reality, and experience that the storyteller produces through the telling. Although narrators are accountable for their stories, narrative researchers treat credibility and believability as something that storytellers

Due to the nature of the research it was important that the participants could not be identified. They were assured that confidentiality would be maintained and any information presented in the thesis would not be attributable to any one individual. The participants were given the opportunity to read the relevant section in the case study before the thesis was submitted for examination. 
accomplish' (Chase, 2005 p. 657). In other words, the stories were believable because they made sense.

It is acknowledged that the storyteller's performances are produced in a particular setting for a particular audience, for a particular response, and shaped by interaction with the audience. In this case, the respondents knew that this was a University $\mathrm{PhD}$ study so the story would have been slanted a particular way, compared to say, a newspaper interview, as the respondents knew they would get access to the transcripts and also the case, once it was written, and have the opportunity to sign off on both documents.

In terms of data treatment, each respondent was interviewed during office hours and they were met on Air New Zealand premises. The interviews were recorded on a digital recorder and then downloaded on to an office computer. The interviews were then transcribed to create Microsoft word document. The transcripts were then coded using the following system: The respondents were labelled A, B, and C respectively. The pages were numbered and each line was counted starting at one at the top of each page. Therefore quotes can be traced back to the transcript by the following reference system: A (respondent) number (page)/line number. For example, $\mathrm{B} 3 / 12$ indicates the quote came from respondent $\mathrm{B}$ and the quote is from page 3 , line 12 of the transcript. In terms of the selection of themes an inductive approach was used. As O'Leary (2004) notes, 'many researchers will look for concepts to emerge inductively from their data without any preconceived notions' (O’Leary, 2004 p. 197). However, a 
deductive approach was used once the themes were analysed in terms of the categories of optimising, reasoning, analogising and creativity. Interestingly, in each of the respondent's stories, there were no grand narratives. It is assumed that this was because the respondents knew that I had written a realist account to the background to their stories, so there was no need for them to reiterate the material.

Whilst the original theme selection was inductive, it is not being claimed that all possibilities have been exhausted. Nor is it being claimed that there has been no reflexivity involved in the process. The stories that were told were constitute the material which could be used to understand judgements and processes involved in action and their consequences, rather than as an attempt to ground the stories as some foundational truth statement. Ultimately what is required is material to enable edifying philosophy to take place.

The next section outlines the Grand Narrative, followed by an analysis of the marketing constructs. The structure of the Grand Narrative begins by outlining a background to Air New Zealand's crisis. This is followed by a description of the various options open to the airline to ensure its survival. A new CEO is appointed and the selection of a new business model for the New Zealand domestic service is outlined. The model, Express Class, is described using the extended marketing mix (price, product, place, promotion, physical evidence, process and personnel) as a framework. Finally, the success of the new operation is described. 


\section{Grand Narrative}

Air New Zealand had come a long way from its former days as Tasman Empire Airways Limited (TEAL) - operating flying boats to Australia and around the Pacific - and National Airways Corporation (NAC), providing domestic air services in New

Zealand. The airline was in a position to grow substantially so the strategic decision was made in 1996 to buy $50 \%$ of Ansett Australia (Ansett) as a means for Air New Zealand to gain access to the Australian market. This move was necessary after the Keating government in Australia revoked an "open sky" agreement that would let Air New Zealand fly on Australian domestic routes.

In June 2000 Singapore Airlines (SIA) made a bid for the other half of Ansett owned by News Corp, offering to pay A $\$ 500$ million, but Air New Zealand had the rights of first refusal so decided to take up the offer instead. Air New Zealand had three directors on the Ansett board but they did not have access to detailed information about the operation of the airline. Whilst Air New Zealand needed to only match the SIA offer, they ended up paying a $40 \%$ premium.

Unfortunately they were forced into a hasty decision without doing due diligence.

One reason for this was due to the actions of the cornerstone shareholder Brierley Investments Limited (BIL). The chairman of BIL, who was also the chairman of Air New Zealand, saw the opportunity to reduce BIL's share in the airline by selling $25 \%$ to SIA, thus leaving BIL with a $30 \%$ equity share.

However, once Air New Zealand had ownership of Ansett the acquisition did not look so positive. For the six month period to December 2000 Air New Zealand 
reported only a $\$ 3.8$ million profit, compared to $\$ 127.2$ million for the six month period the year before. The Chairman, Sir Selwyn Cushing, stated in March 2001 that the January and February figures for the year showed further deterioration in profitability. The poor performance of the company was attributed to intense competition in the Australian market with new low cost entrants, Impulse and Virgin Blue, initiating massive price cuts, as well as the falling Australian dollar putting pressure on costs.

By April 2001 Ansett faced a crisis. Ten Boeing 767 jets, said to be the oldest of their type outside North America, representing 25\% of Ansett's capacity, were grounded by the Australian Civil Aviation Safety Authority (CASA). The authority also demanded that Ansett prove by May 4 that all Ansett planes were safe, otherwise the company would lose it operating license. Unfortunately the grounding took place over the Easter holiday weekend leaving thousands of passengers stranded or facing long delays.

CASA blamed management instability for the maintenance problems of the ten aircraft. It was reported that the grounding of the Boeing 767 aircraft cost $\$ 8$ million in lost revenue, aircraft leasing and other expenses, with market share dropping by $10 \%$.

To make matters worse, all was not happy in the Board Room. The Chairman was due to retire in November but under the Company's Term of reference he needed to be replaced by a New Zealander. The Singaporean and Australian members on the Board wanted him to be replaced by Dr Rose, a New Zealander living in Australia, 
and they wanted to move the head office to Melbourne. Also, there was certain disquiet within the Board about the handling of the grounding of the Boeing 767 Ansett fleet.

Another problem was SIA's reluctance to back the purchase of cut-price rival Virgin Blue, a move designed to preserve market share. They didn't believe Virgin Blue could survive in Australia but when they come to the same conclusion as the rest of the board members, six months later, it was too late as Virgin Blue was doing so well it rejected Air New Zealand's offer.

In the meantime things were getting worse for Air New Zealand. By May 2001 there was a $\$ 700$ million-plus short term hole in its balance sheet. The company's debt equity ratio was at around 59\% leaving little room to take on additional debt. A deeply discounted rights issue was needed to inject some cash into the airline, but the company could not sell its shares offshore. Singapore Airlines, who already had a $25 \%$ stake, were eager to gain greater control but strict ownership provisions in the multilateral air service agreement prohibited further foreign ownership.

Although Air New Zealand was making a loss the company still paid an imputed dividend. BIL, based in Singapore paid only 15\% withholding tax while local investors paid either $33 \%$ or $39 \%$ tax on the 9 cents per share dividend.

\section{Options}

Ansett Australia was losing \$1.6 million a day so decisions needed to be made. There were a number of options open to the board. The first was to allow SIA to 
raise its stake to $49 \%$ but this would only raise $\$ 466$ million which was well short of the $\$ 2$ billion required to replace Ansett's ageing fleet and would also require government approval. The second was to sell Ansett Australia to SIA but this contravened the Australian ownership rules which deemed SIA's 25\% share in Air New Zealand by de facto meant that they already held $25 \%$ of Ansett.

BIL was not happy as both options would mean it ended up with a diluted share holding, which was undesirable, given that they wanted to exit the industry. To compound matters, they did not want to put more money into a rights issue. Under the ownership rules every dollar obtained from the overseas market had to be matched by a dollar from the local market, and with BIL not willing to invest further the market was too small to raise the necessary cash.

The Labour-led coalition government took a long time to make up its mind whether to allow SIA to increase its share holding to $49 \%$. The consequence of this indecision meant that the airline's shares plunged dramatically. Originally SIA offered to pay $\$ 1.31$ for each additional share issued but as time went on and it learnt of the situation with Ansett, they dropped their offer to 67 cents, then withdrew their offer completely. Australian Prime Minister John Howard was also involved in the talks, being especially concerned with whether or not SIA should be allowed to buy out Ansett.

By September 2001, following a significant downtown in performance leading to unsustainable levels of losses, Ansett was placed into Voluntary Administration. Air New Zealand reported a $\$ 1.43$ billion annual loss after writing down $\$ 1.32$ billion 
from its Ansett investment. Air New Zealand was itself on the verge of bankruptcy. On the 4th of October 2001 the Air New Zealand Board, its major shareholders and the New Zealand Government announced a new proposal which provided a capital injection of $\$ 885$ million from the New Zealand Government giving it $83 \%$ of the shares which meant BIL dropped from $30 \%$ to $5.5 \%$ and SIA reduced its share from $25 \%$ to $4.5 \%$.

\section{Airline Industry}

It needs to be remembered that Air New Zealand was not the only airline having problems at this time. September 112001 put the world airline industry in turmoil. Hardest hit were the United States carriers as they suffered a drop in travellers of up to $20 \%$, but airlines around the world suffered a loss of patronage. They responded by cutting fares, laying off employees, reducing airport facilities and eliminating meals on some flights.

However, to add to the strain, the airline industry had been undergoing major structural change over the last decade. There were four main drivers of this change. The first was the changing nature of domestic and international airline regulations. For Air New Zealand, this was evidenced by the November 2000 open skies agreement between Australia and New Zealand, where amongst other things; any authorised airline was able to operate domestic services in Australia and New Zealand. The second driver was the emergence of global competition between airline alliances. As a consequence of the first driver, deregulation of airline markets put pressure on airlines to seek cost reductions and to look for efficiencies. Alliances provided a means to seek efficiencies through seamless travel and marketing 
initiatives as well as price and schedule coordination. It was also recognised that global alliances had the potential to involve partners in joint purchasing of fuel, catering services, and possibly aircraft. It was also possible to seek rationalisation of ground handling staff. Air New Zealand became a member of the Star Alliance, the biggest airline alliance operating at the time, as they realised joining an alliance would provide cost benefits as well as consumer benefits.

The third driver was the growth of Value Based Airlines (VBAs), which brought new competition into the market place. No-frills, point to point airline operators, pioneered by Southwest Airlines in the United States, and easyJet and Rynair in Europe were a new threat to the traditional airline operators as they operate on a low cost strategy. This is achieved by offering a reduced range of services, for example, no pre-booked seating, a single class cabin, with food and drink paid for by the passengers on the flight, as well as flying point to point on short haul routes, and utilising the same type of planes. For instance Southwest Airlines only uses Boeing 737 aircraft. Additional savings are gained by not providing baggage transfers to other airlines. These airline operators also use a ticketless booking system and rely on the internet for sales. VBAs were able to cherry pick the profitable routes so putting pressure on the traditional airlines, which were unable to cross subsidise from the other routes within their networks not flown by the VBAs to compensate their high cost structure. The response of the traditional airlines was to have their own low-cost subsidiary. For example, Air New Zealand had Freedom Air, operating within New Zealand and on the trans-Tasman route as direct competition to the new entrant Kiwi Air operating from a base in Hamilton. Another example is Air Canada who developed Jazz and Tango as low-cost subsidiaries. At this stage it was 
uncertain that the traditional airlines had the ability to manage two very different types of operations. The real fear was that they may very well cannibalise their own sales.

The fourth structural driver has been the rationalisation of ticket distribution, as it is typically the fourth largest expense after labour, fuel and aircraft. Cost reductions can be achieved when airlines use their own internet sites and call centres. On a world wide basis this has lead to travel agents receiving less in commission, consequently their place in the value chain was modified significantly.

\section{A New Leader}

By October 2001 there was a reshuffle of the top executive team. Gary Toomey, the Chief Executive who was formerly the Financial Director at Qantas, resigned and more than half of the top executive team left, including the Chief Financial Officer, the Group Finance Vice-President, and the General Manager of Operations.

In November a new board member, John Palmer, was announced as chairman, and in February 2002 Ralph Norris was appointed as Managing Director and Chief Executive of the newly recapitalised organisation. Having spent over 30 years at the ASB Bank - the last 10 as CEO - this was a change of industry, but one where he could continue his enthusiasm for embracing technology in business. Although on the Board of Air New Zealand throughout the Ansett debacle but having retired as a CEO due to ill health, he saw the opportunity to put his knowledge into turning the airline around. 
"From my experience as an independent director of the company, I know the scope of the problems that have to be overcome, and I look forward to leading Air New Zealand management and staff to produce the solutions [that] are needed to rebuild the core strengths of the company and develop new competitive advantages", he said (Air New Zealand Media Release 13 February 2002).

From the start of his term as CEO, Ralph Norris had two issues that needed to be addressed. One was the latest financial forecast and the other was the staff climate survey. The financial forecast identified a $\$ 40$ million negative movement in the profitability figures from the forecast year ending 2002 and the culture of the organisation was poor. Only $29 \%$ of staff had seen fit to partake in the survey, and $90 \%$ of them had no confidence in management or whether the company had a strategy going forward.

\section{A New Business Model}

Air New Zealand operated three core services, each serving customers with different needs. The domestic service, the most profitable, operated solely in New Zealand, whereas the Pacific service operated in Australia and Pacific Island Flights, and the Long Haul operation consisted of flights over five hours. The decision was made to start restructuring the domestic service by implementing a new business model.

Extensive research revealed that customers did not value all of the in-flight frills that came with Air New Zealand's full service domestic offering and expressed their willingness to trade off some of these for lower fares. As already noted, globally, carriers like Southwest, easyJet and Ryanair had successfully introduced 'no-frills' flights, seemingly under the mantra of "charge only for what you deliver, and only 
deliver what you charge for'. But Air New Zealand was committed to remaining with the Star Alliance and providing seamless travel around the world, so was not prepared to trade off everything in this manner. Therefore it was not just a matter of copying some other airlines 'best practice' (See Appendix C). The new model was a hybrid of other airline models but put together to suit the Air New Zealand operation.

The strategy was to increase primary demand for the domestic service, that is, increase the rate of purchase by existing customers (get them to fly more often) and increase the number of new users. In other words, target people who normally wouldn't travel by air but instead use their car, bus, train or inter-island ferry.

"Our aim is to encourage people to fly, with fares that are strongly competitive with rail and car travel. Our customers told us cheaper fares were their priority - they also told us they were willing to trade off business class and full meal service in return for such dramatic price reductions." (Ralph Norris Air New Zealand Media Release 1 November 2002).

Air New Zealand had a competitive advantage in the domestic market due to the frequency of flights on the main trunk route between Auckland, Wellington and Christchurch, and also its extensive network throughout New Zealand with its subsidiaries Eagle Airways, Mount Cook Airlines, and Air Nelson.

The above competitive advantage was enhanced by the collapse of Qantas New Zealand in April 2001, leaving thousands of domestic travellers seeking alternative flight arrangements. Qantas New Zealand was formerly Ansett New Zealand which 
was a fully owned subsidiary of Ansett Australia between 1987 and 2000. When Air New Zealand bought Ansett Australia, Ansett New Zealand was sold in order to comply with regulatory requirements. The new company, Tasman Pacific Airlines, operated as a Qantas franchise under the Qantas New Zealand brand.

\section{Express Class}

Radically different from the then current service, the new product was called domestic Express class. A useful framework to understand the new service is the extended marketing mix, namely product, price, place, promotion, process, physical evidence and personnel. 
Product:

The tangible aspects of the service offering were changed as follows. The business class seats were removed from the Boeing 737 fleet thereby making a one class service, consequently increasing the number of seats from 122 to 136 . This meant that available seats on the domestic network overall increased by $12 \%$. Full meals and drink selections were replaced with a biscuit, tea, coffee, water and a give-away plastic cup. Also, newspapers were no longer offered, but the inflight magazine was retained. In contrast to Value Based Airlines airport lounges were retained and upgraded as customer research had clearly indicated these aspects of travel were still valued.

Air New Zealand added a $10^{\text {th }}$ Boeing 737 to expand the main trunk schedule to cope with the anticipated growth in passengers. The extra plane came from the subsidiary Freedom Air which was now focusing on the Queensland market as it was thought it was positioned too close to the new domestic Express. Another ATR aircraft was ordered for Mount Cook Airline, a subsidiary of Air New Zealand, to increase the services between Christchurch, Rotorua, Queenstown and Dunedin, and Air New Zealand took over the 'Whisper Jets', BAe 146s, from the failed Qantas New Zealand operation.

\section{Price:}

With the express service fares were lowered and simplified with the removal of advanced purchase or minimum stay conditions. The airline provided just everyday one-way low fares whether passengers travelled during a peak business flight, the school holidays or mid-week in the middle of the day. On the main trunk route fares 
dropped on average by $28 \%$ with the cheapest flights on offer dropping by $50 \%$. According to an Air New Zealand press release, the large reduction in airfares on the main trunk routes was possible due to the operation of the Boeing 737-300 fleet as capacity could be increased more easily and costs could be lowered. However, across the domestic network fares dropped by an average of approximately $10 \%$. Frequent flyers could continue to accrue Airpoints on qualifying fares,

With the strategy of encouraging more people to fly more often, the promise was for twice as many fares to be offered in the lowest price bracket. Three different types of fares were offered in the new fare structure. The Smart Saver fares were nonrefundable and non-changeable, consequently it was a "use it or lose it" fare. Frequent flyer airpoints were not available on this fare. Flexi Saver fares were the mid-range fares with the opportunity to accrue frequent flyer airpoints. The fares were non-refundable, but changes to your booking were possible by paying a change penalty plus service fees and any difference in fare price if the original fare was not available. Changes could be made at anytime before the day of departure, after which it became a "use it or lose it" fare. The final fare structure were Flexi fares which gave the passenger complete flexibility as they could be changed right up until departure, with no change penalty to pay, although the difference in cost of the new fare, if applicable, would be incurred and a service fee would be charged.

To give some idea of the price difference between the three structures, comparable fares from Auckland to Wellington one-way would be $\$ 89$ for a Smart Saver, $\$ 169$ for a Flexi-Saver, and \$256 for a Flexi fare. 
Air New Zealand calculated that the top 500 companies in New Zealand would save up to a combined total of $\$ 40$ million in airfares annually, whereas the small and medium sized enterprises were expected to save $26 \%$ on average annually. However, as part of the low cost strategy discounts to large companies were withdrawn but incentives for sales targets were retained for certain large companies.

\section{Distribution:}

To offer travel at as low a cost as possible, Air New Zealand separated the airfare from the cost of distribution and offered its lowest fares online, as this is the channel with the lowest costs. In other words, passengers were able to gain a lower fare, within any of the schedules, by booking on the internet. However, if a passenger was to book a domestic fare through a travel agent there would be a $\$ 10$ surcharge for the use of the Global Distribution System. Additionally the Air NZ service centre would charge a $\$ 15$ service fee, per one way journey, no matter how long the flight, to recuperate the extra cost of providing personalised service. The travel agents were at liberty to charge their own service fees. Also, commissions to travel agents for selling Express service fares were cut from 5\% to zero but incentives for reaching sales targets were retained.

From only $4 \%$ of domestic online sales before Express Class, it was hoped for a target of $70 \%$ of domestic sales to be made via the internet was set.

\section{Promotion:}

The communication strategy for promoting the Express service identified four main audiences that needed to be targeted with specific messages. The first was Air New 
Zealand staff. Apart from a staff newsletter outlining the change, more than 3,500 employees from around the world participated in a series of group workshops to understand and embrace the strategy. During the workshops the new brand positioning statement 'Being there is everything' was revealed (refer Appendix D).

The second audience was that of the general public. Two new advertisements were created on the theme 'Being there is everything'. (See Appendix D). One advertisement featured a businessman trying to make contact by phone with a fisherman in Southland but after repeated unsuccessful attempts he decides to fly south and greet the fisherman on his boat. The second advertisement showed two grandparents on a flight to meet their new grandchild.

The third audience were travel agents. This involved a two prong strategy. The first part was to talk face to face with senior executives at the different travel houses explaining the strategy in depth, and the second part was to utilise Air New Zealand's existing sales staff to communicate the changes directly to travel agents in their area

The final audience was large public and private organisations. Letters were sent to organisations outlining the new strategy and indicating that existing contracts were no longer going to be honoured. This was backed up by corporate sales representatives visiting their clients, and with the help of modelled data, showing how the organisation would be better off under the Express service. 


\section{Process:}

The drive for internet sales meant that Air New Zealand spent considerable time and effort ensuring that their IT systems and internet interface could cope with the increased load. Also, the anticipated additional passengers could have resulted in increased costs of extra staff at airports, but again technology had a key part to play. Following closely on from the launch of the Express service Air New Zealand introduced self-service check-in kiosks in main domestic airports (refer Appendix E) allowing customers travelling with electronic tickets to print their own boarding pass. Customers were also able to use the kiosk to select their seats and indicate if they had bags to check in.

\section{Physical Evidence:}

Physical evidence in the service environment is an important means to convey service expectations to customers. Following on from the introduction of the kiosks, the check-in area at Wellington and Christchurch airports had to be re-organised with specific counters, along with allocated queue areas, for four different types of customers. One counter was for passengers with international connections, as they could be checked-in for their connecting flights. The second group of counters were for domestic passengers who needed or wanted to check-in with an airline representative. This was necessary for passengers who did not have an electronic ticket. A third counter was allocated to Koru members and gold card members (gained through frequent mileage points) and the last group of counters were for passengers who had obtained their boarding pass from the kiosk but needed to check their bags for the flight. In theory, the idea for this counter was that there would not be a queue as there were a number of counters and the process was quick. Auckland 
domestic terminal only had three distinct groups of counters as there was no need for an international connection counter.

Personnel:

The most significant change to personnel was that the number of cabin crew on the Boeing 737 aircraft was reduced from four to three members. This was made possible as the cabin crew's duties had changed with respect to the meal deliveries. All the crew needed to do with Express service was to hand out pre-packed cups (with sugar, milk, napkin, biscuit and spoon packed inside) and water, and pour tea or coffee. Previously the service involved the delivery of a complimentary meal and bar service.

\section{Success}

November 2003 marked the first year anniversary of the Express Class service. The growth in domestic travel had been phenomenal, producing a record for the company. Over 6.1 million passengers were carried, which represented an increase of 1.1 million passengers (a $22 \%$ increase). This meant that the passenger load factor averaged $75 \%$, which represented a 8 percentage point rise. Bookings on the Air New Zealand website had risen to approximately $40 \%$ and the web kiosks had risen to a monthly uptake in November to $50 \%$ in the main centres.

The results three years after the introduction of the Express service showed that compound growth was approximately $40 \%$. Primary demand had been stimulated by increasing the Passenger Load Factor from $67.4 \%$ to $76.2 \%$. This comprised an increase of capacity, that is, Available Seat per Kilometre (ASK) from 3,638 million 
to 4,281 million, and an increase in traffic, that is Revenue Passenger per Kilometre (RPK) from 2,453 million to 3,264 million.

By November 2005 online bookings were well over 50\% and self check-in at peak times has grown to $60 \%$. With positive customer feedback, domestic market share was up by $6 \%$ from $74 \%$ to $80 \%$.

In summary, Air New Zealand knew it had to be bold and innovative in order to survive. In 2005 Air New Zealand won the prestigious Phoenix Award from Air Transport World magazine. The award recognises an airline that has achieved 'a commercial rebirth through a life-changing transformation'.

\section{Analysis}

The above narrative can be classified as a romance. The evil forces are that of Brierley Investments (BIL), Singapore Airlines (SIA) and to a lesser extent the New Zealand government. These forces are overcome by a new team at Air New Zealand who successfully reposition the domestic service. Was there a hero? The new CEO, Ralph Norris, is mentioned and the last paragraph indicates the he left the organisation in a sound financial footing. In this respect, the narrative is that of a Quest.

In terms of Gergen's plot analysis it can be seen that the narrative starts with a regressive plot but then takes the form of a progressive story. The first section of the story is about the financial collapse, whereas the second section outlines the 
turnaround strategy, leading to the conclusion, which represents the final progressive part of the narrative.

Three underlying structures of the grand narrative have been identified. The first underlying structure is the realist position taken by the author. The author has taken secondary data and written a narrative based on an objective view, written in a style that implies the story is based on fact, and therefore is true. The author has taken a position of authority and written in a clinical, straight-forward descriptive style. This style of writing can be witnessed in Harvard business cases, where a problem or issue is presented and the CEO is often presented as a hero, from which students are expected to learn from such a case.

The second underlying structure is that of rational economics. What is meant by the term rational economics is that the narrative is based on the assumption of rational behaviour and the function of a business is to maximise profit, and thereby increase shareholder wealth. This is evidenced in the first section, which indicates how different players have helped erode the share capital and profitability of Air New Zealand, and the conclusion summarises the success of the repositioning in financial terms.

By adopting the above approach certain issues have been left out of the narrative. One important issue is that of the customer. This is interesting as the topic of the case has marketing implications but the basic tenet of the marketing concept is ignored. Other voices have also been ignored, for instance, that of staff. The repositioning had implications for staff at the check-in counters and cabin crew, as 
well as sales staff that had to renegotiate contracts with travel agents and large corporate accounts. Also, the need for retaining a national carrier was implied but not debated at the time of the near collapse. This is an issue that has national strategic implications but the debate was not publicly aired.

A third underlying structure is that of the marketing mix $^{33}$, which has been imposed by the author. Whilst this is a useful framework to analyse marketing phenomena, it does put a certain constraint on the narrative.

In summary, the Grand Narrative is a monovoiced story based on secondary data obtained from newspapers and magazines, which adopts a realist, objective standpoint with a bias towards presenting financial data. From a postmodern perspective, one can begin to ask what information has been omitted. Would other voices offer different stories, or at least stories with a different perspective? The next task of the case analysis is to examine stories from three respondents who were involved in the decision making surrounding the launch of the domestic Express. In terms of plot analysis the three respondents' stories are that of a drama based on romance as each story has a good ending. Using Gergen's typology it is a progressive story.

\section{Respondent A}

Plot summary

\footnotetext{
33. The standard marketing mix is a framework that can be used by marketers to make decisions that will affect the relationship between the firm and the customer. The mix, commonly called the ' 4 P's of marketing', comprises price, product, place (distribution) and promotion (communication). This has been extended by McGrath (1986) to accommodate service marketing by adding an additional $3 \mathrm{P}$ 's, namely process, people and physical evidence.
} 
The viewpoint of Respondent A was the communication of the new proposition. The task was to assure the New Zealand public that Air New Zealand was now fully functional and that they had a new offer that was to the publics benefit. The task was to move from a rational appeal based on price and airplanes to an emotional appeal which was centred on the customer. Therefore the proposition was developed that concentrated not on the flying experience but about the outcome of that experience. In other words, the proposition was about 'being there', whether it be for business, family, sport, or recreation. To achieve acceptance of the message internal communication was deemed just as important as external communication, so the Board and staff, especially cabin crew as they had dealings with the public on a daily basis, had to be convinced of the benefits of the new communication strategy.

\section{Themes}

Respondent A's narrative was centred on the communication strategy that was adopted to launch the new domestic express. The introductory part of the narrative was used to establish the need for the repositioning, which can best be summed up as a barrier to entry. 'Now we had a real sense of urgency around February-March because we had a very clear indication that Virgin Blue were about to launch a domestic operation within New Zealand and clearly saw the opportunity to launch a domestic operation particularly on our trunk route Auckland-Wellington which to us has always been a jewel in the crown of the way that we manage our domestic network. So we had to act incredibly quickly and we had to act as much on the emotions of our customers as on the rational benefit to our customer' (A2/4). 
In resurrecting the airline and making sure it was profitable in the future, there was a need to protect the most profitable part of the business, namely the domestic network, and in particular the main trunk route between Auckland, Wellington and Christchurch. These particular routes were the most profitable with a large capacity so could easily be cherry-picked by a new entrant.

The narrative has one major theme, that of creating and implementing a communication proposition. There are two subsidiary themes, namely internal marketing and market research. The major theme will now be explored in detail.

\section{Creating and implementing a communication proposition}

The main communication problem was to get the message across to the New Zealand public about the new low fares whilst acknowledging the customer has to give something up in return. 'The main driver was an awareness that strategically we were about to take something away from customers and not be immediately obvious in the way that were trying to give something back. So what we were giving back was a significant price reduction and the ability for customers to do whatever they wanted to do with the extra cash that they would normally have paid for the airfare. What we were taking away would have more emotive value' (A1/3).

However, getting the customer to a point where they would embrace the strategy was difficult as the company did not want the customer to think they were paying the price for the company's financial failure. 'We had to be very careful in the way that we positioned what we were doing with domestic Express so that it didn't look like a 
dumbing down, and it didn't look like a punitive measure to punish the customer for our financial difficulties in the previous year' (A1/14).

The task, therefore, was to move the customer from a rational appeal to an emotional appeal. 'So we had to think very clearly about how simply this could be communicated. If we communicated this purely as a rational business methodology, it would fail because the customer simply wouldn't connect and the customer wouldn't respond in the way we wanted them to' A2/11). A two pronged communication strategy was developed with the above point in mind. Stage I was a brief campaign, which involved a one-off press advertisement and a television advertisement called 'Runway', which ran for three weeks. 'So those two ads were deliberately short term, one-off bursts and were almost apologetic in tones that said to the New Zealand public that everything that had gone on before saying please bear with us and put this all behind you. And without those ads we could never have moved the positioning. We had to speak as a business talking to its public first, before we could then move on to the consumer position and it was the consumer positioning where we took a big leap of faith in saying this is not an airline talking to its customer this is simply a pure customer positioning around 'Being there is everything' (A3/5).

The Runway advertisement allowed the business to move their key proposition. 'So we had moved away from the airline talking like a business to one that was saying this is about the customer and this is about a customer proposition but we couldn't have reached this second stage if we hadn't gone through that first almost mea culpa 
phase of almost saying we got it wrong, we're determined to get it right and this is what it now means for you' (A3/8).

The second prong of the strategy, Stage II, was to talk about the new domestic Express service by using an emotional appeal. This involved creative decision making as it involved a new way for the company to talk to customers. Therefore the advertising agency was briefed about the new communications strategy. 'Now every single piece of marketing activity we had every featured had always featured an airplane - it was almost a standard pack shot as the hero, that was the core and a lot of emotive branding of the airline in the past and we said, we are going to strip all of that out and we are encouraging you as the agency to think of taking a proposition to market that doesn't feature an airplane, doesn't feature our product at all. And the agency took a long time - firstly to come to terms with the fact that we were going to make all of these changes to domestic, secondly that we were going to make all these changes by the $31^{\text {st }}$ of October, so that within six months we would completely, radically have rethought the domestic product and reduce fares by $30 \%$. And the third challenge for them was to say that we are going to do this without talking about ourselves as an airline, but we were going to talk about ourselves purely as a service provider and not as an airline. So it took them about a month to get over that - the fact that we were actually going to follow up on that and to genuinely do it' (A3/21).

The development of the advertising slogan 'Being there is everything' involved creative input. 'And it was the account planner who said that the whole proposition needs to revolve around the customer and he came up with the line 'It's actually all about being there'. It's not about the airline getting you there, it's not about...it has 
to be first person. It's not about us being clever driving you there, it's all about you being clever getting to the destination, getting there more cheaply, most cost effectively and more often than ever before', and he turned the whole proposition around to a customer proposition from a business proposition' (A4/13).

This process took time and but finally the creative proposition clicked. 'So the reason I' $m$ coming onto this, and I think this is pretty critical is that we took it absolutely right back to basics and this was the defining moment. When we drew this chart on a whiteboard we developed 'Being there is everything' within 15 minutes of drawing this chart. Somebody drew this on a board and we instantly went 'now I understand where we are heading' (A5/15).

An analogy was used to obtain the necessary emotional image. The model we used was a really powerful model to me at the time - and frankly there's only so much you can reinvent from an advertising perspective that the model that I tried to capture was one of...I had only arrived in New Zealand in January 2002 and I sat for the first month taking up the job and one of the first things you have to do is try to immerse yourself in what's happening in advertising at that time and I watched reel after reel after reel of ads, and there was only one ad that I thought was any good at all in the country at the time. It was a Telecom ad - it was a girl in a dingy London flat, quite depressed, getting emails from her mother of the pohutukawa tree that she used to play on as a kid on Cheltenham beach. I sat and watched this ad and thought bugger me that is brilliant, absolutely brilliant because it isn't about technology, it isn't about how great email is, it's about someone sitting in South London missing the colours of the Pohutukawa tree. And I thought, that's where we need to be. Because 
what I loved was the balance between the rational and the emotional and they were sending out loads of rational messages' (A6/18).

This type of thinking allowed the advertising agency to develop a story board that was about enabling people to get to a destination for emotional reasons. 'So we kept thinking, and once the planner had said get from A to B, go and see somebody, go and see something, the creative then said - it was a guy called Michael O'Sullivan who is now a creative director at MC - brilliant, genius guy - and he was basically saying, what is my equivalent of the girl in the London flat? What was this clever piece of kit that suddenly transforms a grey surrounding to a really bright surrounding. The first ad that came off the back of that and is still far the strongest ad because it was just the simplest was the idea of the cynical old grandfather who can't hug a photograph. That was literally how the conversation went, what about the old bugger who doesn't get emotional about seeing pictures of his first grandchild and the first time he actually picks up his grandchild he bursts into tears' (A7/12). 'You know, to me it was just one of the purest creative processes that I've ever been involved with, because the proposition was so simple and so clear that building pictures off the back of it was actually a piece of cake and the biggest challenge was actually stopping them coming' (A7/21).

The importance of linking the rational and emotional messages is underscored by the following quote: 'We were very much driven by the girl in the London flat, in saying that could we ever produce an ad that delivers that message - that domestic flights are really that cheap - as cleanly as that ad did. And in some ways I think we probably did it better, but that was the inspiration and it was all about this link 
between emotional and rational that we hadn't understood, that we were so busy surviving that we couldn't see that we had been spending all our time in the rational territory and doing really dull price led advertising. So we said take yourselves out of it, we won't show a plane, we won't show a price, we won't even tell people how they got there. All the creatives would allow me to do - because I was the typical client saying 'got to have an airline, got to have an airline' was to say we will give you 3 seconds in 60 seconds to say how an airline made a difference. And there is a moment where the grandmother is crying her eyes out on the flight, and the flight attendant comes over and gives her a box of tissues and I thought that was brilliant because it was an airline that actually got you there and that's all I wanted to be able to say - that somehow the airline made a difference, made it a better journey' $(\mathrm{A} 8 / 15)$

From this one advertisement three other advertisements were produced around the same story board idea for different target audiences. 'And we produced these three ads that cut across these ages but operated on the same principle, that until you get on a flight, nothing actually happens' (A10/16).

\section{Internal Marketing}

A subsidiary theme to the main theme of developing a communication proposition is that of internal marketing. Whilst it is essential to communicate the new product to the general public, it is also important to get staff support to the new product positioning and the advertising campaign. 'The hardest part was more internal than external. It was actually getting people in the company across the fact that we were about to spend money that we didn't have - we had lost $\$ 1.8$ billion' (A9/17). 
Two important internal groups needed to be taken into consideration. The first was that of the board of directors who wanted the advertising campaign to be more aggressive about the importance of New Zealand having a national carrier. 'The board wanted us to go out and say Air New Zealand, we're back and we're in your face and your New Zealand national icon and I had to push back internally against all of that and say no, what we are going to show is customers. That was the hardest bit.' (A10/3) Winning the approval of the board of directors was essential for funding. However, winning the support of the second group, that is, the general staff was important because of their daily contact with the customers. This was especially true of cabin crew and corporate sales staff. Initially the general staff were sceptical of the communication proposition as they could not understand why only customers were being shown. 'Again, they were saying why aren't we out there hammering home price, why aren't we hammering home competitive advantage, why are we simply portraying customers?' (A10/8) However, once they had seen the ads and the community at large were positive about the product offering they came around to seeing the value of the proposition.

The biggest group of staff who had the most difficulty with the advertising campaign were the engineers and pilots who wanted a pack shot, that is, a picture of the aircraft in New Zealand colours. 'It was pretty hard and it was pretty bold to try and get that understood by engineers and pilots who want to see more aircraft in those ads' (A10/20). This is understandable as they conceive travel, that is, the aircraft experience as being the most important rather than simply 'being there'. For them this was a huge conceptual mind change. 
Due to the radical changes that needed to be embraced by Air New Zealand personnel, it was important to obtain staff support. The impetus for this was the holding of an internal road show, called 'Changing Our Future' (COF), which was attended by 800 staff. 'It was an event in mid-July 2002 and we had produced the Runway ad - the prelude to all of these Being there is Everything ads and we had pulled 800 staff together at a venue near the airport and I said that somebody had produced this 60 second ad, it had cost us a quarter of a million to make, which in advertising circles is nothing to produce, and we wanted to show this ad to demonstrate that we are moving forward, that we have got momentum, we have simplicity, that sort of thing. I showed them those values and I said that we've got pride, we've got quality, we've got passion and we now need to show the New Zealand public that we've got momentum and we're capable of simplifying' (A11/11). After the advertisement was shown it was noticed that half the audience were in tears, which was an emotional response to the enormity of the situation.

The road show was seen as a turning point for the staff. It made them realise the magnitude of the problem and the need for them to accept the challenge to turn the airline around from being severely in debt to being profitable with ongoing positive cash flows. 'The very first session was a two-day session and the first day was spent entirely on saying 'this is where we are, we know you are angry about it, get stuff off your chest. You've got Chief Executive Ralph Norris who is new to you. He is here, and is going to be here for the two days, you've got Chief Operating Officer Andrew Miller, he's here. We've got all of the senior managers of his team, you can rant and rave, tell us how bad things are and we can tell you how dire the operating 
environment is. We can tell you that not only has Ansett gone bust, that Swiss Air has gone bust, Sabina has gone bust, all the American carriers are in Chapter 11. We can tell you how grim the situation is. We'll spend the first day doing that, but we are going to spend the second day with the new management team, and we are all new and absolutely bloody committed doing something about it. So a new ad position will help but it's not the answer. The answer really is a commitment to service, the answer is a commitment to lower prices, the answer is making some difficult choices like taking meals and business class off domestically, and the answer is having a joint belief that we are actually going to be strong enough to trade our way out of the current operating difficulties. And that was quite cathartic, you know was partly a huge release for the staff there, but partly also in taking a different position in the marketplace and losing this corporate arrogance. And we kept saying to people "We're not the largest airline in New Zealand, we're the $36^{\text {th }}$ largest airline in the world". Completely different mindset, and not a mindset that New Zealanders were used to thinking about their airline' (A13/17).

\section{$\underline{\text { Market Research }}$}

Another subsidiary theme of Respondent A's narrative was that of market research. The amount of market research can be best summed up by the following quote. 'We didn't do a huge amount. We did a lot on the product. We didn't do a lot on the campaign' (A15/5). In terms of gaining information about the product, the issue was centered upon the question whether passengers would be prepared to give up a certain amount of in-flight service for a price reduction. 
The airline had received feedback about their inability to relate to customers. 'We got very clear views from customers that we were coming across as we were trying to project pride and what people were getting out, what the perceived experience was nothing to do with pride it was alienation and the actual experience of dealing with our cabin crew, of dealing with check in processes was actually arrogant, inconsistent and impersonal' (A4/20).

'Your staff are rude, you're arrogant and you've actually lost touch with the travelling public, so don't give me any of that expensive marketing message because it's bullshit. Give me a message, give me a proposition that I can actually buy into' $(\mathrm{A} 4 / 4)$.

The majority of the market research utilized focus groups. 'We had to take people through by that step by step process. We had to tell them first what we were going to take away, we had to tell them second what we were going to replace it with and then say to them, if you were having to make that trade-off, would you be happy to make that trade-off? And again, that was quite a rational process but had to be pulled through to emotion, of saying 'so will you fly more often as a consequence?' $(\mathrm{A} 15 / 15)$.

An interesting aspect of the market research was that focus groups were also undertaken with staff. 'I think we probably did as many focus groups with our own staff as with the customers. So we actually took as many flight attendants and pilots through those focus groups. Because what we realised fairly early on was that flight attendants actually spend far longer with our customers than anyone in the sales force 
so in terms of getting an assessment of what customers would or wouldn't accept. They are a much, much better barometer than anyone in sales. Because they actually live and breathe the customer, they're accountable. You know, it's them that the customer shouts at, not necessarily the sales force' (A16/1).

Whilst some market research was conducted on the product there was little carried out on the advertising campaign. What was interesting to note was the use of informal feedback. In terms of the advertisements' cut-through and penetration the barbecue test was used. 'And it was only I think when their friends - you know the BBQ test - when friends were saying to them 'I saw your ad last night and it really struck a chord with me and it really resonated' (A10/10). 'People talk about the 3 degrees of separation. In a small market, you don't need to do much market research. We never once researched this campaign after it aired. Never once tested it, because I never needed to. We were getting so much response, so much feedback there was never any point testing it' (A21/4).

\section{Respondent B}

\section{$\underline{\text { Plot Summary }}$}

Narrative B's plot is about pricing and revenue management. The narrative tells how the airline needed to come to grips with some hard data and do the necessary analysis before making the changes centred on price reductions. Whilst the direction was to maintain the domestic airline's profitability there was a need to establish a perceived fare reduction in the market place. The narrative outlines how this was achieved by adopting a different model of pricing and implementing a modified revenue management system. Two themes were apparent in this narrative. The first is 
centred on the pricing issue and the other theme was about taking the project on as a personal crusade. These two themes will now be explored in detail.

\section{Pricing}

The main theme of Respondent B's narrative was on the topic of pricing. Air New Zealand, following the demise of Ansett Australia and financial crisis that followed, needed to look at different models of airline operation. 'I guess this sudden exposure to low cost carriers and their cost models, their pricing models and their distribution models, and just the way they turned a lot of the industry down-under on its head, and certainly a number of people in Air New Zealand, including myself, went through some pretty gory times. And I guess it kind of illustrated for many of us how vulnerable our organisation is, or was' (B1/6).

In particular, the worry was that Virgin Blue, a low-cost carrier that had started-up in Australia with good results, might enter the New Zealand market. 'So there was a long process internally within the company looking at what our options were to address that situation. And that really started a whole bunch of different scenarios that people looked at. But it was the genesis, if you like, for Express, recognising that we needed to do something different, and address the sort of competitive cost/price issues that a low cost carrier could have done in New Zealand' (B2/8).

When undertaking a price review it is important to understand the most profitable price segments. 'Without a shadow of a doubt, the reason that Air New Zealand makes money domestically is because of business people. It's not because of the leisure customer who buys a $\$ 50$ fare going to Christchurch’ (B5/10). 
However, it is not about dropping prices across the board. What Respondent B realised was that the pricing issue was a segmentation issue. 'But dropping prices, doing it carefully, targeting the right market segments and managing the process, maybe might get us to breakeven. And all the other things have to fall into place as well in terms of cost savings to actually make it the right thing to do' (B5/22).

Within Air New Zealand the pricing team was separate from the revenue management team. Whilst the pricing team sets the selling price for different routes, noting that each flight has a range of prices, commonly called buckets. The pricing team takes into account relative route pricing and the pricing of competition. The revenue management team on the other hand is interested in the yield that is produced from each flight. This is measured simplistically by Revenue per Available Seat Kilometre multiplied by load factor, and then divided by Cost per Available Seat Kilometre. Inventory for each flight, that is the number of seats allocated to each bucket, is used to control yield. Therefore, to adopt a pricing strategy for domestic Express there was a need to do some pricing and revenue management analysis.

'So I went away and did some analysis and number crunching, forgetting the way that revenue management was done at the time which was all very much black box, highly sophisticated, expensive systems with very complex optimisation algorithms and so forth and sort of throw that aside for a moment and go back to the old way of doing things which was very much like low cost carriers which is quite prescriptive, quite targeted, to say we want to sell this much of this much and that much of that 
much on this day, that day, this month, that month, this flight, that flight and try and build a relatively unsophisticated, not optimised, simple management process that said we are prepared to sub-optimise for longer term benefit in terms of trying to win customers over that and I guess we coined the phrase at the time a term called 'bias to volume' if you like so that you have a revenue target and you can get it be either squeezing people or you can get it by making more seats available and having a bias to volume and we sort of coined that phrase about making sure that people feel comfortable about their purchase decision and therefore buy more often which is a slightly different take on the revenue management process. And by doing that I guess we made sort of a paradigm shift almost from the old way of doing pricing and revenue management to a new way of doing pricing and revenue management which said 'we will un-encumber our pricing systems away from the old way of very restrictive fares with round-trips and Saturday stays and advance purchases' and you know in the old way for someone to get a cheap flight someone had to jump through all sorts of hurdles and so forth to actually get it' (B7/13).

This analysis involved calculations on elasticity as well as growth in the market. 'A lot of price elasticity work around the different segments of the market, a lot of work to understand what the growth forecasts were going to be like and how we were going to manage that and how the revenue was going to change etc etc' (B8/16).

The low-cost model was analysed and the company took advantage of talking to an employee of Easyjet to get an understanding of their pricing strategy. 'Their customer segmentation was simply a time-related one. Depending on when you book, you get a different price. So that's well understood. We did have a meeting 
with Ray Webster of EasyJet - a former Air New Zealand guy - who simply reiterated all of that, but the fundamental piece of information that he gave to us as a business that allowed us to make the next step was getting management and marketing comfortable with fact that guess what, their lead-in price, they didn't actually sell very much of' (B9/17).

Initially the thought was that the airline would adopt a pricing strategy where there were a lot of cheap fares and few high fares as per the following model:

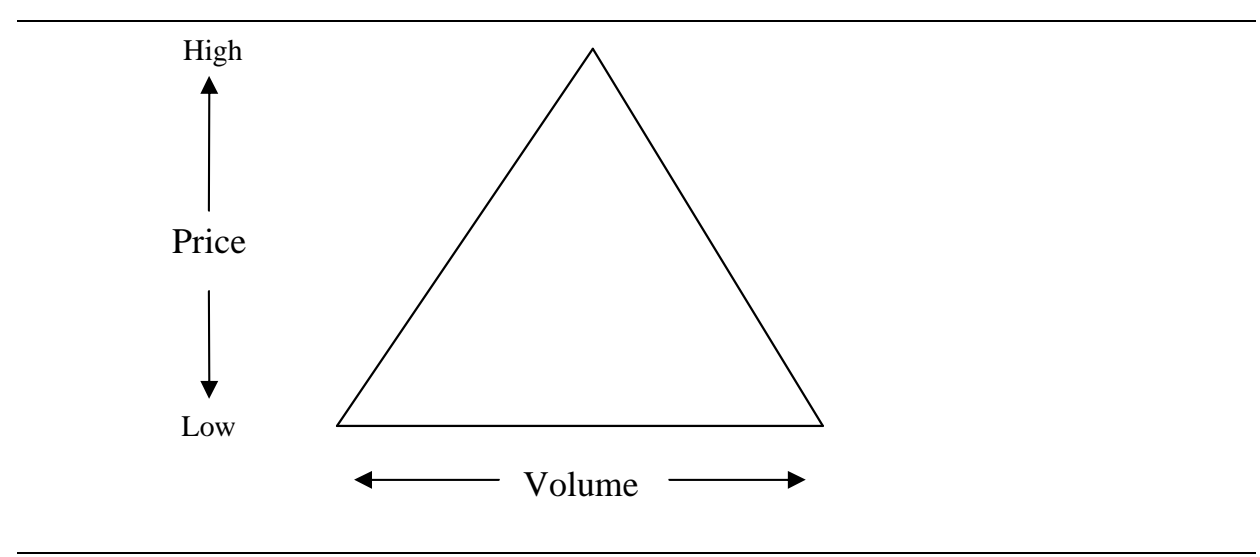

'This structure is a disaster and this is what Freedom do today in fact. And it's a disaster for them because they basically sell nothing but low fares' (B11/21). However, Air New Zealand learnt that there was a better way to approach the pricing model. 'The pricing would be like that - very little at high and loads at low. What Ray actually showed us what that their pricing diamond was like this' (B10/9). 


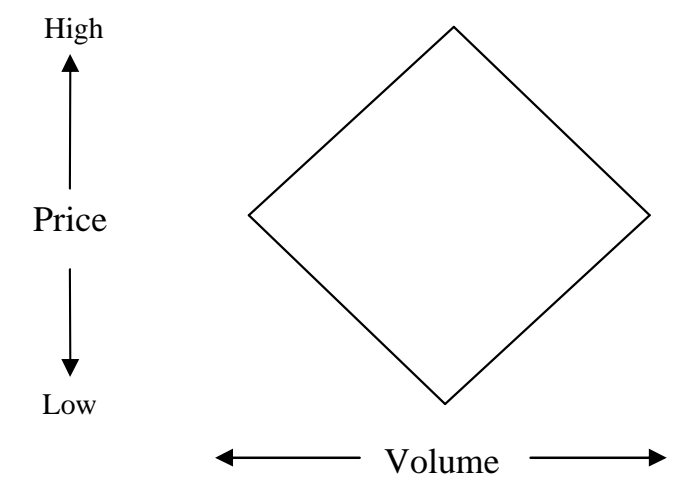

This is compared with the traditional model, which is still used by some airlines.

'Lots of people still do this. It says these guys at the top, they are price inelastic, they don't care what they pay they just want a ticket so that they can go tomorrow, do a day trip or whatever. They don't like paying loads of money, but they can. These guys down here don't like to pay loads of money but give them a price they can and you can sell loads of it provided these [high] guys aren't actually diluting down' (B11/16). 


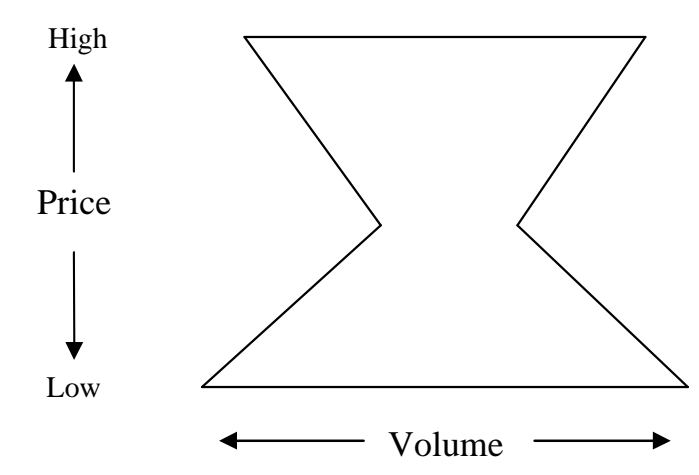

Finally the pricing model was developed and put forward for adoption. 'This is to say that we will be prescriptive and still a little restrictive in terms of our rules to make sure we don't get too many people buying the low fare. We will have a price, and we had a lot of discussion with legal about how much we would need to make available to satisfy fair trading conditions etc so you know there was lots of talk about whenever we have a low fare out there we need to make sure we have at least $5 \%$ of seats available. It doesn't sound like much, but 5\% of the Air New Zealand network is a huge amount of seats. So generally speaking people can still get cheap prices and you can focus on days of the week, times of day and if you are going on a Saturday evening there are masses of those seats available and if you go on a Monday morning there's none. That kind of thing. And that allowed us to conceptually move to a different level about what we wanted to do with the pricing model and the revenue management model' (B12/3). 
However, by adopting this new approach, the old revenue management system had to be modified. 'The expensive part of PROS is all these complex optimising algorithm systems, the less expensive part of it is a tool, or work bench, which allows you to prescribe certain sorts of things. Now, in the old way of revenue management, $90 \%$ of it is the black box, and $10 \%$ is the toolbox where the analyst adjusts things. In the new way of doing it, that flipped around completely to $90 \%$ manual intervention, $10 \%$ optimisation' (B13/17).

Once the system was modified the pricing and revenue teams were able to do some projections. 'Then taking that customer set and because of this kind of pricing segmentation we were doing, segmenting them out into various categories of priceelastic leisure customers down the bottom, price-inelastic business customers at the top and effectively using our old pricing segmentation model as a customer segmentation model on the premise that most of the people buying the really high fares were inelastic. Some of them were elastic, but were forced to buy those fares because there was no other option. But $95 \%$ of them were price-inelastic. So using that as a proxy, trying to create a customer segmentation from that which would allow me to map different levels of elasticity, different price discounting models to drive different outcomes and sort of going through an iterative process of trying to understand what we wanted to deliver - in terms of a discount - how we wanted to manage that in terms of the number of people we were going to sell at it, and how that was going to affect different levels of customer within that model. To a large extent it relied on my intuition about all of that and I have to say, I felt decidedly uncomfortable for a long time about my projections because there was a lot of lead 
time from announcing it to getting the effect in from the yield in the overall revenue etc and whether or not we were going to go from being a very profitable domestic business to losing our shirts' (B15/22).

The constraining factor in developing a new pricing strategy was that there was a directive from the CEO that the domestic service had to remain profitable. 'Ralph was absolutely adamant throughout all of this that we were not going to reduce the profitability of the domestic operation' (B19/13). The difficulty in managing the process was that board had expectations that seemed to be in conflict. 'It's almost like they wanted their cake and they wanted to eat it. They wanted to discount and wanted to give everybody the fare they wanted but they also wanted to give the shareholders exactly the same return as they were getting. It was a nightmare conceptually to get that analysis and that together' (B19/17).

\section{$\underline{\text { Crusade }}$}

The underlying theme of Narrative B is what could be called a crusade. From the perspective of Respondent $\mathrm{B}$, he wanted to become involved in the project and take ownership of the problems surrounding pricing. He had returned from working with Ansett Australia and their was a strong personal motivation to learn from the experience. 'And you know, the experience taught me a lot and certainly taught the organisation a lot as well, and I guess for a long period of time, post-Ansett, there was an inquisition going on within Air New Zealand as to what went wrong, and why, and most particularly how we could protect ourselves from that happening in our own backyard' (B1/6). 
At the time there were different projects being undertaken by Air New Zealand looking at their service offering. 'And there was a long bunch of projects at quite a high level, at a very high level, that was moving down the path of all sorts of weird, wonderful and crazy ideas about what Air New Zealand was going to do and become. I came back and saw some of this going on, and got quite nervous or anxious about some of the things that were being talked about, including for example, turning the whole of domestic New Zealand into Freedom [Air], which would have been an unmitigated disaster for the company' (B2/11).

The decision to get involved was the belief that a pricing strategy was being developed by senior management without them having a good grasp of the cause and effect consequences that would impinge across the business if certain scenarios were adopted. 'And so when these high level projects are going on and you're not involved in them, it gets quite disconcerting for our area to think that someone is making broad assumptions about pricing, or broad assumptions about revenue management, broad assumptions about competitive market share, capacity share, distribution impact and all that kind of stuff without engaging our area' (B3/4).

Therefore, with this going on Respondent B decided to get involved. 'And I managed to insert myself in those projects because I was deeply worried about what people were going to assume, particularly around my area at the time - pricing - but also about the revenue management areas, the network planning areas, and effectively the kind of analysis and research about what might happen if they took certain courses of action. So I got myself engaged in it to keep an eye on those 
things and you know at the start of all of this, the premise was that Air New Zealand needed to become a low cost carrier domestically' (B3/15).

The first big challenge was to get people involved in the project to re-think the implications of adopting a low-cost model. 'So it was a bit of challenge at the start to try to get people off the page of low cost carriers' (B4/12). This education role meant that staff had to see the complexity of the pricing decision. 'So I felt I had a significant contribution to make there in terms of making sure that we were able to deliver on that promise, whilst also, I mean, there was a certain naivety in the company at the time that we just drop prices and everything would be okay' (B5/2).

Following on from this the next step was to develop a pricing strategy that would be acceptable to those involved in the project. 'So I had that responsibility to take that expectation of what the company wanted to achieve and what the public wanted to see and actually convert that into something that was going to work for the company' (B5/17). In doing this there was a need to steer a path between meeting the expectations from senior management who were viewing the situation idealistically and the staff in revenue management who were committed to the traditional way of calculating a price strategy. 'On the one hand you had the idealistic senior management types who perhaps didn't understand the detail of how things worked the cause-effect if you like - and on the other hand you had the established mentality within my peers that what we were doing was the right way to do it. There are still people in my organisation that felt we needed to stick to the old way of doing things. And that meant the old fare structures, the old revenue management principles and 
that this is the way that other full service airlines do it and we need to stick to our guns' (B6/4).

In an attempt to satisfy both parties Respondent B attempted to blend the two approaches and find a middle ground. 'I was caught in the middle between trying to fulfil different sets of criteria - and so I guess to a fault - I went in initially with a proposition of a fare structure that was kind of a blend of the two processes, where we had some cheap fares, relatively unrestrictive one-way type fares, and then a whole bunch of quite structured restricted fares that fitted the old revenue management practises of the past' (B6/12).

Working through the issues Respondent B came to the realisation that he needed to come up with a new approach. 'Now, I won't say that I had an epiphany or anything like that in the process, but after much to-ing and fro-ing and debating about what was best, it finally clicked in my mind that instead of looking at it solely from a pricing perspective, which was my mandate, I needed to take it to the next step and say actually our revenue generation as a business is driven by both the pricing set, and the revenue management set. So what I needed to come up with was a new way of doing both' (B7/4).

Once he had developed the basis of the new pricing strategy it was necessary to sell the idea to the other participants, which involved talking through the issues with the revenue management team, as well as market planning, so they could see that they needed to do things differently. 'So it was quite a lengthy process to try and get both sides onside' (B8/25). 
The personal crusade took commitment and determination but paid off in the end. The company relied on the Respondent's analysis and judgement, trusting in his ability to find the right solution. 'But it worked and it was a bit of a leap of faith from the company in a lot of ways and I guess people trusted my analysis more than maybe they should have done - I don't know' (B16/17).

\section{Respondent C}

\section{Plot Summary}

The narrative for Respondent $\mathrm{C}$ began with an introduction concerning the need for the airline to reappraise its situation in light of the financial crisis and the problems with Ansett Australia. It was noted that there was a changing of the guard with a new Chairman of the Board, a new CEO and management team. The narrative then concentrated on two issues concerning the launch of domestic Express. The first issue was an outline of the political nature of the decision and the second was about the changes in the ticket distribution. An interesting point was the role of the CEO who acted as facilitator by listening to the different arguments but then making the final decision. The narrative concludes by stating that whilst the domestic Express is successful it now needs to be modified to accommodate new customer perceptions.

The two themes in the narrative, namely the political nature of the decision making and the distribution issue will now be explored in more detail. 


\section{$\underline{\text { Political }}$}

The momentum for change was that the Government had approved the recapitalisation of the company which meant the company had to think clearly about future plans. 'And then in due course, Ralph coming in to be the boss. So we were at a time where, I would say, it was 'backs against the wall' and it was a fight for survival situation. Because we had been saved by the recapitalisation, but I think it was pretty evident to everybody on the management side that that was a one-off saviour, and we now had to re-engineer the business and rebuild it into a sustainable model - because there wasn't going to be a second bail-out! - and we had to produce a five-year business plan for the government to justify the bail-out' (C1/10).

However, in deciding the plans it became evident that there were two factions, one representing a more traditional approach and the other pushing for a more radical approach based on a low cost carrier model. 'At the time, of course, there was conflicting views within the company as to what the strategy should be. And I guess you could say that there was a power struggle to a degree, in that we had Andrew Miller who was heading up part of the company, including Strategy \& Marketing, and then we had Norm Thompson heading up very much the Sales side of the company. And the two of them initially had differing views on the strategy and Ralph had to be the arbitrator on many occasions on how the strategy would work. And we probably had a lot of conflict in the early stages, which in some ways you could say "old airline thinking" versus "new airline thinking". There was a group of people who had been around the company for a long period of time who were saying, “this won't work or that won't work or we've got to do it this way or we've got to be very traditional" etc and then we had another group who were being very proactive 
and progressive, saying "we've got to try this", "we've got to do this" etc. So to get the concepts of the Express idea through internally was very challenging because there were some major changes that people who had been in the organisation for some time were very reluctant to accept' (C1/17).

This conflict also had ramifications concerning the brand positioning of Air New Zealand. 'And we also had this real conflict, I think, between the brand and the new product because the Air New Zealand brand had always been positioned as being at the quality end of things, full service etc and then here we were bringing in this new product which was a much lower spec than what we had, but we still had this Air New Zealand brand positioning out there' (C2/22).

Given this conflict there were people in the organisation who were disengaged and to some extent alienated. This was primarily because a new board and new senior management team had taken over and was more concerned with the future of the organisation than past successes. 'That we had to look to the future and not to the past. I think there was a bunch a people over that period had become alienated. I've worked a long time at this company, and there were a lot of people who had put a long time into this company, a lot of energy and emotional involvement, who felt a bit aggrieved that what they had done wasn't recognised. With the new management saying the past was dead and most of it was wrong so we are now moving to the future. So I think a lot of people got alienated with that' (C5/8).

Also fuelling the conflict was the insights many employees saw regarding the implementation of the strategy. On the one hand the reasons for change were clear 
and there was a general understanding of the model as it was presented but there was a hesitancy that the company could be throwing out the baby with the bathwater.

‘And over time, many of Norm's group could clearly see why we had to change, but also felt that some of the change was too radical. Probably again because they were the people who had to implement it and that was really challenging for them' $(\mathrm{C} 3 / 16)$.

In the end it was the CEO who was able to settle the conflict and facilitate decisions. 'And I saw Ralph as MD being very much an arbitrator. He was a good leader in that he was able to diplomatically manage the two camps and then come down and make what he thought were the right decisions, without getting too much into the conflict himself' (C4/12). 'He very much sat back out of the day to day tussle and then made the final calls' $(\mathrm{C} 4 / 17)$.

\section{Distribution}

The second theme in Respondent C's narrative was that of distribution. One of the bigger issues that needed to be decided regarding domestic Express was the issue regarding changes in ticket distribution. First, the decision to increase internet sales as this distribution channel required a lot of development. 'And of course there was some pretty new stuff, like distribution via the internet, where Air New Zealand had only really been scratching the surface on that, and Express required a huge leap to improve the internet distribution and develop off the lower fares and so on' $(\mathrm{C} 2 / 13)$. 
Second, and perhaps the biggest debate, was centred on the commission paid to travel agents. What was of concern was the impact the decision not to pay commission for domestic travel would have on international travel revenue, which relied primarily on travel agents bookings. 'For example, the decision to go to zero commission for travel agents was a mammoth internal debate because we had traditionally distributed the product through travel agents, and many people were not convinced that the internet was ready to take the channel shift that we needed, and that the customer wasn't ready to move away from travel agents to a direct position. And also because we were reducing the travel agents' income domestically we would get a backlash on the international services' (C3/19).

The issue was further compounded by the fact that Air New Zealand had set itself up in direct competition to travel agents by having 0800 telephone sales, travel centres (shops), and now the internet. 'The net result was that most travel agents earned less money from Air New Zealand and at the same time we set ourselves up in very direct competition, because we had a pricing advantage on the internet' $(\mathrm{C} 6 / 13)$.

The outcome with the above changes meant that the relationship with travel agents changed. 'So the relationship between Air New Zealand and travel agents is now very business-like, whereas before a lot of it was based on genuine friendship and genuine relationship - now it is very business-like and Air New Zealand has made no secret of it. I think one of the great things with this whole change of strategy was our transparency. We didn't go out there and bullshit anybody, we told them as it was. And that was a new thing for Air New Zealand because unfortunately we had a reputation, and rightly so, that we always only told $3 / 4$ of the story and there was a $1 / 4$ 
that we never quite told people. Whereas we suddenly had this new strategy under Ralph of being absolutely transparent. And the travel community found that a bit hard at first, to think that we could be telling the whole story, but they ended up accepting it' (C7/17).

However, the other area within distribution was the question of rebates for corporate customers. 'Then the second part was the corporate rebates where our corporate position in simple terms had been structured around the more money you spent with Air New Zealand the better deal you got. So people like Telecom and Fonterra got rebates, whereas the small guy didn't. And under our new model, it was 'one size fits all' - whether you were a twice a year traveller or 20,000 sectors. Our sales team did a fabulous job in getting out to the corporate customers and getting them to understand the new fare model, and that they way it worked they would be better off. We had to place certain guarantees with some companies but it all worked out okay. But it was pretty hard to convince a Procurement Manager at a big organisation who had dealt with Air New Zealand for 10 years and had always enjoyed a 30\% rebate that he would no longer get a rebate, but he would be better off. It was quite hard to get them across the line on that. So that was a big challenge for our sales team and we had the two prongs of it out there to convince people that earning less was better for them' $(\mathrm{C} 6 / 21)$.

Whilst the above two issues in distribution required a big change for Air New Zealand the overall outcome was satisfactory as internet sales increased above expectation, the travel agents reorganised their respective businesses and started to 
charge service fees, and the large corporate organisations welcomed the new low fares.

\section{Framework}

The case has used sensemaking to explore narratives to understand a complex marketing decision. A grand narrative was written using material from secondary data. In contrast, three different narratives were obtained from individuals involved in the marketing decision, and the themes from these narratives were explored. Seven themes in total were analysed. In Chapter Six a framework was introduced that allowed for a placement of themes on a paradigm grid. This framework has been adopted to explore marketing decision making (see Figure 10).

The $\mathrm{x}$ axis of the framework is split been monophonic and polyphonic. The case outlined in this chapter started with a Grand Narrative, which was monophonic. To counter that position, three alternative voices were sought. The y axis is based on Wierenga and Van Bruggen (1997) decision making activity. The conceptual dimension comprises the optimisation activity and the mental modelling activity, whereas the associative dimension encompasses the two activities of analogising and creating. 
Figure $11 \quad$ Narrative Themes applied to Air New Zealand

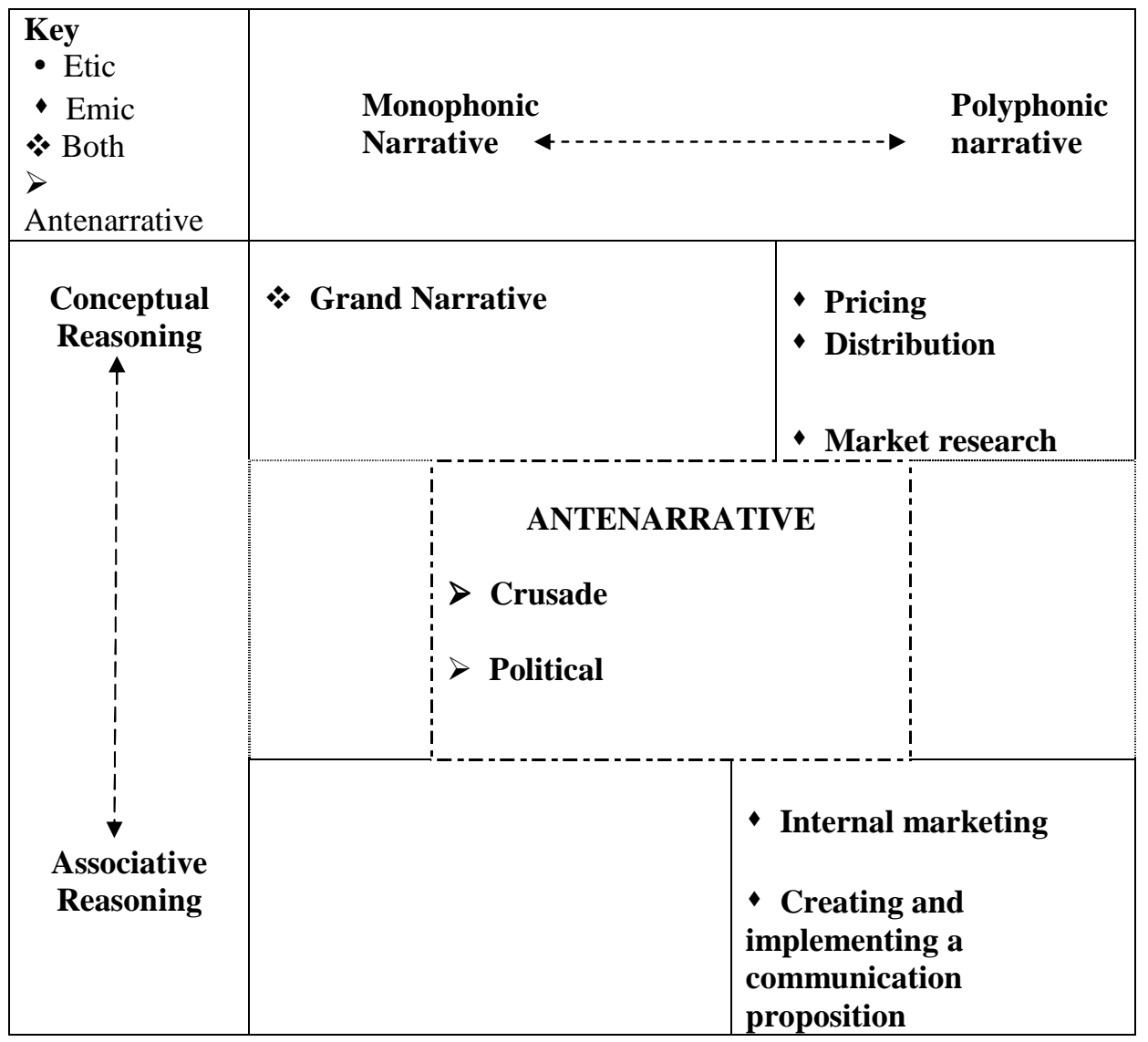

Source: Adapted from Boje, 2001

The Grand Narrative is monophonic and is based on a conceptual type of activity, whereas the seven themes vary in terms of their dominant reasoning pattern. The theme 'creating and implementing a communication pattern' primarily involved associative type reasoning. Analogising was evident in terms of adapting other television commercials, and creative reasoning was evident in terms of determining a story board. The 'internal marketing' theme was bordering between conceptual 
modelling and associative type reasoning whereas the 'market research' theme involved conceptual reasoning.

The theme that involved optimisation type activity was that of 'pricing'. The use of models and quantitative analysis of data was evident in the narrative. 'Distribution' was a theme that used conceptual type reasoning, with a hint of associative type activity to make sense of the information.

However, two themes, namely 'crusade' and 'political' stand outside of the framework and as such can be seen as antenarrative. They show another side to the Grand Narrative and pose questions about agendas being worked out in the organisation. This is contrary to the rational model posited by Wierenga and Van Bruggen (1997). The implications of this will be explored in the next chapter.

The framework, as outlined above, is a good visual stimulus to compare the different themes with the Grand Narrative. The implications inherent in the framework will be discussed in detail in the next chapter.

\section{Evaluation}

The question arises as to the trustworthiness of the respondent's narratives. According to Denzin and Lincoln (2005), trustworthiness equates with validity, but before the matter of evaluation criteria can be addressed, certain issues need to be clarified. 
First, it needs to be remembered that this is a postmodern narrative, consequently the evaluation criteria will be different from positivist or modern interpretivist criteria. Second, the question of trustworthiness takes on a different hue as foundational knowledge is not being sought. Third, the stories depend very much on the context that they are given.

Having raised these three issues, it is now necessary to address the issue about evaluation criteria. Below is a table outlining the evaluation criteria of positivist versus interpretivist research. Whilst it is not necessary to explain each dichotomy in detail, it is important to show how each of the interpretivist's criteria can either be matched or adapted to account for neo-pragmatism, which is based on affirmative postmodernism.

\section{Table 4 Research Evaluation Criteria}

Positivist Indicators

Objectivity

Reliability

Validity

Generalisability

Reproducibility $\underline{\text { Interpretivist Indicators }}$

Subjectivity

Dependability

Authenticity

Transferability

Auditability

Source: O’Leary 2004 
The question of subjectivity addresses the third issue raised above. Stories depend upon the context they are given, as noted in the methodology section. Therefore, the subjectivity of the respondent's stories has been acknowledged and as foundational knowledge is not being sought, subjectivity does not pose as problem, especially as the goal of the research is to show praxis in action, with the intent that edifying philosophy can take place.

The case study acknowledges that reliability is not possible, but the documentation contained in the methodology section has been handled systematically and is welldocumented so dependability has been obtained, but in light of the qualifications made in the methodology section.

Authenticity requires that there is some truth value to the case. Whilst the case openly acknowledges multiple truths, and that each story is authentic only in the sense that it contains narrative logic (Hopkinson.2001). In other words, the storytellers were believable and the elements related in plausible ways so the story made sense.

Certainly the case findings are not generalizable, nor was that the intent, given the anti-foundational stance of neo-pragmatism. However, the issues raised by the case are transferable to some other contexts. Lincoln and Guba (1985) have suggested that the term transferability is used fro qualitative research, rather than the term generalisability. The issue of transferability is important as neo-pragmatism is antifoundational and refuses to seek for essential characteristics, so the question arises what can be learnt. According to O'Leary (2004), transferability is an indicator of 
applicability. Can the lessons from the case be applied to different settings? For this to happen, a highly detailed description of the research context must be given so those reading the account can decide upon the applicability. It is important to note that the responsibility is on the reader to decide the transferability of the case.

The final indicator is that of auditability. Whilst a description of the process by which the case material has been obtained has been given, it is possible to audit the case. However, given the neo-pragmatism research protocol it is questionable whether this is necessary s the goal is not to find grounded truth, but to provide material for discussion to take place, with the intent of developing understanding.

\section{Conclusion}

This chapter has used a case study based on the launch of Air New Zealand's domestic Express to exemplify the use of an affirmative postmodern perspective. In particular, the use of narrative theory linked to organizational sensemaking was used to explore the complex decision making used to substantiate the launch. Initially a grand narrative was developed which showed the decision as being functional and rational. However, other voices with different narratives were used to counter the grand narrative. Each narrative had its own perspective and providing individual themes, namely creating and implementing a communication proposition; internal marketing; market research; pricing; crusade; distribution and political. Each of the themes were explored and placed on a framework to compare and contrast. The task of the next chapter is to discuss the implication of this case in light of the overall thesis. 


\section{Chapter 8: Conclusion - Contribution of a New Discourse}

\section{Introduction}

The thesis has reviewed the philosophical underpinnings of the marketing management school and has argued that the discipline should adopt the linguistic turn in modern philosophy, which involves a postmodern stance. Upon reviewing the topic of postmodernism it has been argued that the discipline should adopt neopragmatism as a research programme. The thesis then outlined a case, using narrative theory, based on Air New Zealand, as an example of the research protocol in action.

Neo-pragmatism argues that there is no final vocabulary and rejects Cartesian dualism. In its place is hermeneutics as an ontology and epistemology, with the aim of understanding praxis. Such an understanding would allow for practical action, based on edifying philosophy. Therefore the purpose of this chapter is to explore the contribution of neo-pragmatism to the marketing management discipline.

The first section of this chapter outlines the ramifications of the linguistic turn in philosophy for the marketing discipline, especially the role of paradigms. The second section explores the implications for marketing research followed by the implications for the education of marketing management. The issue of marketing theory in light of the new approach is the examined, by a discussion of the art versus science debate. The next section examines the issue of relevance and relates it to the concept of praxis. The exemplary case is discussed and the issue regarding marketing praxis is examined. Finally, the chapter discusses the limitations of the 
research, the issue of reflexivity, and future research, followed by concluding remarks.

\section{New Paradigm}

Over the past two decades there has been a call for marketing to adopt a new paradigm. Within this timeframe there has been discussion as to why such a paradigm shift is needed, and different methodologies have been put forward as possible candidates for this new position (Hackley, 1998; Burton, 2001; Easton, 2002).

The drive for a new paradigm is based on two broad issues. The first is the changing emphasis within marketing from a transactional focus to the building of relationships (Buttle, 1994; Gronroos, 1995). Also there has been an interest in other types of marketing organisations, for example, network marketing (Achrol, 1997). Other forces have been the growth of the knowledge economy and the drive towards mass customization (Day, 1999). The second issue is the growing uneasiness within the marketing discipline that the present way of conducting research has led to disappointing results (Brownlie \& Saren, 1992; Brown, 1995; Saren, 2000; November, 2004). However, postmodernism has challenged the dominant logic of marketing (Brown, 1994; Firat \& Venkatesh, 1995) as outlined in Chapter Four. It is this challenge that this thesis has addressed, by exploring the philosophical underpinnings of marketing management and arguing for the adoption of the linguistic turn in modern philosophy. 
However, the concept of paradigm needs to be explored as it is used extensively in the literature. It would seem that in the marketing literature the word 'paradigm' is being used in two very different ways. The first use of the word is taken to mean framework. What is argued in this case is that the framework of the marketing mix (commonly referred to as the 4P's of marketing) is outdated due to changes in the marketing arena as outlined above. Whist this certainly appears to be the case, it is argued that marketing, like most social sciences, attempts to make sense of current practices, and as marketing is a dynamic, amorphous type of field, new perspectives are needed to accommodate new phenomena, for example, the growth in internet marketing. Therefore, this use of the term 'paradigm' is not contentious. In fact, the creation of frameworks and new perspectives within a discipline, whether it be arts, sciences, or social sciences, is a basic raison d'etre. For example, philosophy has a long lineage of philosophers who have argued for new perspectives, from Socrates, Plato, Aristotle to Descartes, Hobbs, Locke, to Hegel, Nietzsche, and Wittgenstein. Within the management literature it is easy to see the impact and influence that frameworks such as Porter's Five Forces, and Kaplan and Norton's Balanced Score Card have had on both the discipline and practitioners.

However, another use of the word 'paradigm' is that employed by Kuhn, and utilized by Burrell and Morgan, Arndt, and Deetz as outlined in Chapter Two. In this sense the word is used to explain the philosophical underpinnings of a discipline. As noted by Burrell and Morgan there are three underlying structures, namely that of ontology, epistemology and methodology. 
Whilst there has been within the marketing literature an abundance of articles espousing the benefits of different methodologies, there has been scant debate regarding the ontological and epistemological dimensions. In particular there has been a reluctance to address the implications of the linguistic turn in modern philosophy. In exploring the impact of the linguistic turn in modern philosophy on the social sciences, and in particular the marketing discipline, the thesis has explored postmodernism and in particular the work of Richard Rorty. From this analysis it is claimed that the marketing discipline does not need to adopt a new paradigm per se, but adopt a new philosophy of social science, that is, the linguistic turn in modern philosophy.

Adopting a new philosophy of social science will result in the adoption of a new research approach. The point needs to be made that the new philosophy of social science, whilst having distinctive ontological and epistemological foundations, may well utilize different methodologies.

The linguistic turn in modern philosophy challenges the logical empiricist view of the world as outlined in Chapter Two and Three. Three main themes arise from the linguistic turn. The first is that any view of reality is dependent on language. The linguistic turn rejects Cartesian dualism and the correspondence theory of truth. The second is the anti-foundational aspect of knowledge, which means that there are many ways to make knowledge claims, consequently there is no truth in an absolute sense. The third theme is anti-essentialism, with respect that there is no finite list of descriptions to determine the criteria of whether something belongs to a group. 
Therefore, what is the implication of the above themes for the paradigm debate?

Whilst the paradigms model insists that there are no privileged positions in terms of research approach, it is constrained by Cartesian dualism. On the other hand, the Deetz framework, 'Contrasting Dimensions from the Meta-theory of Representational Practices', as outlined in Chapter Two, is a framework that is an improvement on the Paradigms as it takes into account the linguistic turn in philosophy, replaces the objective/subjective dichotomy as it is based on a false dualism, and allows for new approaches such as critical theorists, feminists and postmodernists.

Following the work of Deetz, it would seem advisable to discontinue the use of the word paradigm as it has many interpretations and can be misleading. Instead it is proposed that the word discourse is used. Following on from above, therefore, it is postulated that different discourses serve different purposes. The dialogic studies discourse as outlined by Deetz was selected to explore in detail as it took into account postmodern thought and allowed for the development of praxis. Following the work of Schön on the Reflective Practitioner, the thesis has sought a way to understand practical wisdom as displayed in the Marketing Management School. By adopting the work of Richard Rorty, the philosophy of neo-pragmatism was utilised as an ontology and epistemology to understand praxis.

Differences between the scientific approach and neo-pragmatism can be seen by the constructs outlined in Table Five. The thesis has argued against logical empiricism as an approach for the marketing discipline for the following reasons. The first is that technique and measurement take centre stage, at the expense of context and 
wider implications. As such, logical empiricism leads to a narrow form of knowledge, with the development of experts who know only a specialised area of a research domain. Another criticism is that scientific approach has become privileged in society at the expense of other forms of knowledge. Finally, marketing, like other social sciences, is seen as dynamic, involving human actors, and unlike the natural sciences, is not stable over time.

Pluralism, on the other hand, leads to other forms of knowledge generation, such as art, literature and music, allowing for different perspectives and insights.

\begin{tabular}{l|l|l}
\hline \multicolumn{1}{c}{ Table 5 } & \multicolumn{1}{c}{ Comparison of Research Approaches } \\
Mode & \multicolumn{1}{c}{ Logical Empiricism } & \multicolumn{1}{c}{ Neo-Pragmatism } \\
Orientation & Cartentism & Pluralism \\
Ontology & Logical empiricism & Hermeneutics \\
Epistemology & $\begin{array}{l}\text { Empistemological Behaviourism } \\
\text { testing }\end{array}$ & Narrative \\
Methodology & Objective Reality & Beliefs based on evidence \\
Truth & Theory & Meaning \\
Knowledge & Prescriptive & Praxis \\
Orientation & Ahistorical & Historical \\
& General & Particular
\end{tabular}


The thesis also supports Rorty's arguments against Cartesian Dualism on the grounds that it is based on a false mind/body distinction. The attempt to establish foundational knowledge by the use of mental constructs, that is, the use of reason, is challenged by Rorty, who claims that knowledge is community based and that there are no fixed criteria to determine science from non-science. Rorty offers Hermeneutics as an ontology, as outlined in Chapter Five.

In terms of an epistemology, the scientific mode in marketing utilizes logical empiricism, which was discussed in Chapter Three. This is in contrast to Rorty's approach, whereby he diminishes the importance of epistemology in the analytic philosophical tradition, where epistemology is seen as the essence of philosophy. Instead Rorty adopts hermeneutics whereby knowledge is a matter of social practice. Therefore, knowledge becomes a matter of understanding social practice where beliefs are justified.

In contrast to logical empiricism which uses hypothesis testing as its methodology, narrative theory has been offered as an alternative methodology. Whilst this is an excellent method to highlight how other forms of knowledge can be generated, another benefit is that it is an appropriate method to understand practical thinking. It is through stories that marketing managers' praxis can be understood. However, it needs to be stated clearly that it is not being claimed that this is the only method that could be used under neo-pragmatism. Discourse Analysis is another choice, as is Grounded Theory. In many respects the choice of methodology is not an issue, as in line with Feyerabend's claim, when it comes to method 'anything goes'. Therefore, it is possible to utilise statistical modelling if it gives rise to greater understanding, 
but not as a claim to foundational knowledge. Unfortunately in marketing the debate has been at the methodological level and divisions made between quantitative studies and qualitative studies, but it can be seen that this distinction is too superficial.

The thesis has argued that the word 'paradigm' is misleading as it is based on Cartesian dualism so should be rejected. In its place the word 'discourse' should be used. The thesis argues for the adoption of neo-pragmatism with an accent on understanding praxis. What does this research programme mean in practice? The next section outlines the implications for academic marketing management research and also for the teaching of the discipline.

\section{Implications for Research}

Given that the main thrust of research for the marketing management discipline is that of praxis, based on neo-pragmatism, the question needs to be addressed regarding the implications for research.

The first implication is that science is seen as being no different from other forms of knowledge generation, such as art, poetry or music. This means that praxis can be understood from a number of sources, not just research based on the evaluation criteria outlined in chapter seven. For instance, a novel may give insights to understanding about markets, exchanges, and marketing relationships that can be useful for purposeful dialogue.

The second point is that the anti-foundational position outlined in chapter five, means that research is not about seeking the truth, in the sense of grounded knowledge. The 
logical-empiricist approach has been about finding the right methodology by concentrating on technique. In contrast, neo-pragmatism is not concerned about methodology but gaining insights which are useful.

The third issue concerns essentialism. Adopting neo-pragmatism as a research protocol means that research will not be about finding the characteristics of marketing terms, for example, what would count for something to be called 'services marketing'.

Finally, neo-pragmatism is concerned with finding material for edifying philosophy to take place, so that understanding of praxis ensues. Such an understanding means that insights into practical actions is obtained, not in a grounded sense, but in the sense that what might work. With edifying philosophy the accent is on discussion to create new ideas and concepts. Therefore the marketing management academic will look at responses to marketing dilemmas to gain insights and topics for discussion. This means that case studies and other means of gathering insights into such dilemmas, such as discourse theory, narrative theory and grounded theory, will be utilised. The main focus is investigating what happens in the market place, for example, what marketing managers do to gain topics for edifying philosophy, rather than do research to find what they should do on a prescriptive basis.

\section{Implications for Teaching}

Given the above discussion, the task of teaching marketing management will be the exploring of cases and ideas, but context will be the most important issue. In chapter one, different topics were outlined within the marketing management school, for 
example, services marketing, not-for-profit marketing, but each with different contexts. To explore different contexts the teaching will need to give examples and seek dialogue with the students to contextualise the information. Discussing critical success factors for an industry is one way to lead such a discussion so that students understand contextual setting.

Another important change is the move away from prescriptive models. As has been cited in the previous chapter, marketing management has used planning models with an emphasis on one size fitting all occasions. The task of the teacher is not to prescribe what the students need to do, but for the students to develop their own praxis. The important outcome for the student is not to understand how to use a prescribed model, but to develop their own dialogue and gain understanding. Therefore, in class it will be about contextualising the issue and discussing alternatives, but not with any definitive answer grounded in foundational knowledge.

The above approach as outlined will not be too difficult for many marketing teachers as they are already doing it to a certain degree. For example, many teachers already use case studies, and the standard text book, for example, Kotler and Keller (2006), Marketing Management textbook has examples and cases for students to discuss.

\section{Theory}

The purpose of this topic is to explore the effect of neo-pragmatism as a research protocol on the topic of marketing theory. A number of authors (Brownlie, 1992; Razzaque, 1998; Saren, 2000; Burton, 2005)) have argued that marketing lacks theory, which is to the detriment of the discipline. Theory, it is argued, is a 
framework or a set of guiding principles that focus a research agenda or is used by managers as a frame of reference to make decisions. The marketing discipline has not developed theory to a large extent but there are a number of discreet examples, such as the product life cycle. As outlined in Chapter One, there are a number of reasons why marketing has not developed theory. One reason is the problem of theory borrowing, which is most evident in the area of consumer behaviour. The main issue is that often theories borrowed are used out of context and applied inappropriately.

Another reason why there has been a lack of theory development is due to the dominant paradigm in marketing. Logical empiricism, which has evolved from logical positivism, has used the correspondence theory of reality to underpin the conception of truth and in particular theoretical frameworks. Such an approach uses theory to explore the relationships between frameworks. Therefore hypothesis testing is central to this approach. Hypothesis development is achieved by determining a gap in the literature and testing discreet variables without any integration into a theoretical framework. The consequence of this is that marketing researchers have concentrated on methodology, paying scant regard to the ontological and epistemological foundations of their research agenda, with the consequence that there is an over-emphasis on technique.

The above discussion raises two questions. First, does the marketing discipline need to develop theory? Second, are there different types of theory? 
Marketing needs to develop theory for two main reasons. First, theory development is a guide to researchers in a given field. A theory allows the researcher to investigate their work, and in doing so allows them to see it as part of a bigger whole. However, the marketing discipline has relied on theory borrowing with often scant regard for developing its own theory. This has been at the expense of the discipline in terms of its status as a subject and its scope. Due to the lack of theory, marketing is seen by other academic disciplines as lacking credibility and acceptance as a legitimate academic discipline (Willmott, 1999). This is reflected in the allocation of research grants by higher education authorities.

Also it is argued that due to marketing's lack of theory, the scope of the discipline is being eroded by other disciplines such as Information Systems (internet marketing), Operations Management (services marketing), and Accounting Management (pricing).

The second reason for developing marketing theory is that practitioners use theory to understand the marketing world and to make better decisions. This leads to a discussion about the different types of theory. As outlined in Chapter One, a distinction can be made between theories 'of' marketing, where a grand theory is developed to cover all of marketing, and theories 'in' marketing, where theory building is undertaken at a specific areas, for example, pricing.

In contrast to the above is the distinction made between prescriptive theory and practical theory (as outlined in Chapter One), where the former is based on ungrounded, rationalistic scholarship and is concerned with applying theory to 
practice with the aim of discovering law-like generalizations. The latter is concerned with intentional activities as these are seen as the foundation of practical theory. The above constructs can be used to develop the following matrix.

Figure 12 Marketing Theories

\begin{tabular}{c|c|c|}
\multicolumn{1}{c}{} & \multicolumn{1}{c}{ Prescriptive } & Practical \\
\cline { 2 - 3 } Of Marketing & Generalisations & Game Theory \\
\cline { 2 - 3 } In Marketing & $\begin{array}{c}\text { Specific } \\
\text { Outcomes }\end{array}$ & $\begin{array}{c}\text { Reflective } \\
\text { Practitioner }\end{array}$ \\
\hline
\end{tabular}

The above matrix can be linked to the distinction made by Hollis (1994) between explanation versus understanding on the one hand, and holism, where the agent is part of a larger system, versus individual, where structures are known through individual action, on the other.

The main argument of this thesis is based on affirmative post-modernism in that there can be no workable Grand Theory. This means that the pursuit of a overarching theory of marketing would be in vain. The question is, given that theory is important as a guide to researchers and as a guide for practitioners to understand the world, how can the process of theory construction be taken seriously. 
Whereas from the logical empiricist point of view, the function of theories is to determine their truth value, the pragmatic implications of theory are still important for the affirmative postmodernist. For the neo-pragmatist, frameworks and concepts are important at the practical level, where dialogue is used to reach agreement regarding the application of praxis, that is, theory-in-use.

'For under modernism, the proper theory should be fortified with years of research, and its application undertaken by yet another culture (the practitioners). In the postmodern context, the primary ingredient of theory is not its data base but its intelligibility, and the very communication of this intelligibility already establishes grounds for its utility. Theory and practice are inseparable' (Gergen, 1992 p. 217).

Therefore, the logical empiricists see theory and practice as independent, based on a dichotomy where the objective is to gain truth statements about two or more constructs. The neo-pragmatists on the other hand see theory and practice as intertwined and the value of theory is based on its utility, that is, theory is something that works, and truth value is of no concern.

In summary, what is argued is that given Rorty's emphasis on praxis and knowledge being generated at the level of the particular situation, the Reflective Practitioner's quadrant is an alternative place to generate knowledge about praxis. Marketing has the opportunity to develop theory at the level of the practitioner relating to individual actions in the workplace. 


\section{Art versus Science}

What will be the effect of neo-pragmatism research approach on the art/science debate? The thesis has presented three themes that would indicate that the problem can be resolved.

The first theme is the claim that the art/ science debate is based on a false dichotomy. The second theme is that marketing is not a scientific discipline, and the third theme is that there are different kinds of knowledge, not just scientific knowledge.

However, before discussing each theme in turn it would be apposite to briefly outline the debate.

At this stage it needs to be reiterated that the thesis is interested in the generation of knowledge in the marketing management school. To understand the dynamics of the decision making by marketing managers research can be conducted which will generate concepts, theories and frameworks. The thought is such research will enable marketing managers to make more informed decisions.

In Chapter One it was outlined that over the last 50 years attention has mainly been given to what would count as a science, with little regard paid to what would constitute an art. Brown (1996) outlined three stages in the history of marketing concerning the debate, stating that the last stage is the anti-science era, representing challenges by postmodernism. The work of Schön can be added to the debate as he claims that 'technical rationality', the epistemology of positivism, is an inadequate methodology to understand practical problems. 
The issue regarding either/or as a dualist proposition was an attempt to find criteria for the foundation of knowledge. The dualism covered the objective/subjective debate, where the former was viewed as being an independent overseer, with knowledge corresponding to reality so it was possible to obtain objective truth. Following this, the debate centred on stating explicit criteria for something to count as a science.

Neo-pragmatism rejects Cartesian dualism, stating that a distinction between beliefs and knowledge cannot be maintained. Consequently there is no objective truth. Therefore it is not a question of science or art, but an acceptance of both dimensions as a means to generate marketing knowledge. This leads to a discussion of the second theme, namely marketing as a scientific discipline.

For Rorty, scientific enquiry is based on an empiricist dogma. The idea that data is linked to a theory of knowledge is unnecessary. Therefore foundational knowledge based on objective truth is rejected. Pragmatism also rejects scientism, that is, social sciences such as marketing utilising scientific procedures as if they are natural sciences. For pragmatism it is not about predicting and controlling the behaviour of subjects but the gaining of meaning and understanding. However, it needs to be noted that Rorty is not against the scientific method per se. It can be a useful method of inquiry but should not be seen as the only vocabulary used to generate knowledge.

This therefore leads to the third theme. For neo-pragmatism there are many forms of knowledge. If there is no distinction between knowledge and beliefs, then scientific knowledge, that is knowledge gained from using a scientific procedure, is no 
different from poetry or art. Therefore, no one voice is privileged and any knowledge is based on a particular situation. There can be no general theory, as outlined in the previous section, but only knowledge in a particular context. Such knowledge is dependent on the language game being used and the participants using edifying discourse to arrive at some sort of agreement.

When the above three themes are taken into account the problem of whether marketing is an art or a science disappears. It has been argued that marketing is not a science in the strict application of the natural sciences, but at a particular level scientific procedures are equally applicable as other forms of knowledge, such as poetry, art of literature.

\section{Relevance}

The central concern of the thesis has been about the marketing management school adopting the linguistic turn in modern philosophy. Therefore, the central question to this section is to ascertain the affect a research programme based on neo-pragmatism would have on the gap between marketing academics and practitioners.

The thesis has argued, endorsing the work of Schön, that the logical empiricism practised by mainstream marketing academics has not helped practitioners. Furthermore, it can be argued that the dominant paradigm has marginalised marketing practitioners in the eyes of their colleagues. Neo-pragmatism on the other hand is concerned with praxis. By providing an ontology and epistemology based on hermeneutics, coupled with narrative theory as a methodology, neo- pragmatism 
provides an insight into practitioner judgement, which can be linked to Schön's reflection-on-action.

However, there appears to be two issues that need to be made explicit in the debate about relevance. The first is the gap between academic research as published in academic journals and the apparent lack of practitioner interest in such journals. In other words, the question can be asked as to the reasons why practitioners do not read academic journals. In light of the above, especially taking into account the work of Schön, and the problems with the dominant paradigm as outlined in Chapter Two, it would seem that the work is inappropriate to the audience. Whilst it may be necessary to have journals that have as a primary audience other marketing academics, in an effort to provide some help and guidance at a professional level, what is needed are a number of journals which emphasise praxis, thereby giving practitioners something of interest. Such journals need to be given high status by academics so that it becomes an inherent part of the reward system.

Whilst it is acknowledged that there are a number of barriers to the transfer of academic knowledge to practitioners, such as writing style and the academic reward system, an important issue is the communicating of findings to practitioners. The emphasis on praxis will help overcome this latter issue as it is maintained that practitioners will have a natural interest in the research. In other words, they will see it as apposite to their professional development as reflective practitioners.

This leads to the second issue, namely that practitioners are in their own right stakeholders of marketing research. Taking a business school perspective, it is 
important to realise that practitioners are in the classroom to gain insights, theories and conceptual frameworks that they can use as reflective practitioners. Therefore research-based teaching, arising from utilising a neo-pragmatist approach will provide students with insights and understandings, which they will find meaningful.

It will be noticed that the question of relevance is related to the development of theory. Taking the position as addressed in the Marketing Theories matrix in the previous section, the Reflective Practitioner quadrant is the basis for the development of theories-in-use that would be useful for practitioners.

Finally, it has been argued that if academics get too close to practitioners then the latter group will set the research agenda. Unfortunately, this argument is based on a false dichotomy. Rather than an either/or situation the difference between the two groups can be seen a continuum, with the role of the consultant on the continuum as well. What is argued in this section is not that marketing practitioners set the agenda, nor consultants for that matter, but rather the research is more insightful for all stakeholders.

\section{Exemplary Case}

The reason for writing an exemplary case was to show how a new paradigm would work in practice. The underlying foundation of the thesis was to explore how practical wisdom can be understood in a marketing management context. The idea of practical wisdom as first mentioned by Aristotle (phronesis) and is described by the term 'praxis'. 
The issue of praxis was highlighted by Schön in his work the 'Reflective

Practitioner'. He suggested that the logical empiricism/positivism was inadequate in analysing and understanding this type of behaviour.

Chapter Three explored the issue of the dominant paradigm in marketing management and came to the conclusion that it was an inadequate paradigm to deal with the generation of knowledge from a practical wisdom perspective. Following the discussion of postmodernism and its influence in the $21^{\text {st }}$ century, the work of Richard Rorty was explored, where he provides a philosophical basis for the understanding of praxis.

The case was based on the ontology and epistemology of neo-pragmatism and narrative theory was used as a methodology. By using the technique of sensemaking respondents from Air New Zealand were asked to reflect on the strategic marketing decision to introduce domestic Express service to their New Zealand service. This is akin to Schön's notion of 'reflection-on-action'.

The aim of the study was to determine how managers made decisions in the workplace. The overall objective was to highlight the notion of practical wisdom from a postmodern perspective. This entailed the seeking of three different voices from the organisation. Members of the project team were used to obtain these voices. The interviews were conducted after a 'rational' case was written, based on material obtained from daily newspapers, business weekly newspapers, and company press releases. The case was rational in respect that is was written from an objective point of view and was a description of what happened. 
The respondents, on the other hand, had different stories to tell, and were not only about what and why the decision was made but also how the decision was made. From the interviews seven themes were analysed to ascertain where they would fit on the Basic Narrative Themes framework.

The framework was based on a difference between monophonic and polyphonic narratives, compared with conceptual and associative reasoning. Whilst three themes, namely pricing, distribution and market research involved conceptual reasoning, two themes, internal marketing, and creating and implementing a communication proposition were based primarily on associative reasoning. This gives some credence to the cognitive classification published by Wierenga and Van Bruggen (1997), who argue that there are four types of distinct activities involved in marketing decision making, namely optimisation, mental modelling, analogising, and creating. However, it needs to be noted that two themes, crusade and political, were seen as being outside their classification, consequently it is argued that such themes indicate a significant handicap to their model. As has been noted, their model is based on a cognitive approach to problem-solving. The case study points to a considerable flaw in their model as it is unable to explain decisions based on emotional responses or politically motivated decisions. 
The case also shows the applicability of the Brownlie and Spender (1995) strategic balance model regarding judgement. The themes outlined in the case can be overlaid on to their matrix as follows:

Cell Name

Cell A creative and Visionary

Cell B Bureaucrat

Cell C Salesman/marketer

Cell D Negotiator
Theme(s)

Creating and implementing

communication problem

Pricing

Internal marketing

Political

According to their matrix, the judgement takes place in cells A and D. However, they do state that there is always a mixture of analysis and judgement, but it is a matter of degree.

Another interesting point is that decision making in the case was conducted as a group project, carried out over a period of time, with group members representing different facets of the organisation. Consequently, different members utilised different skill sets, whether it be conceptual and/or associative reasoning. Assuming the model has some credence, the above has implications for the assigning of members to a project team. Depending on the problem being assessed, it would seem that a balance of decision making skills is optimal.

The illustrative case study also indicates that theories in use were employed by the respondents. For example, the use of price as a barrier to entry without sacrificing revenue from significant segments of the market was utilised. Another example was the use of price elasticity to increase primary demand, that is, attract new customers 
who might travel by other means, such as by car, train or ferry, and also increase the rate of purchase, that is people flying more often.

The case highlights the use of hermeneutics based on neo-pragmatism and the use of narrative theory to develop an insight into a marketing phenomenon. The case was not concerned with obtaining 'the truth' regarding marketing decision making so that situations could be explained, (that is, show cause and effect and hence develop a generalisation), but to gain the insights to develop meaning and understanding based on praxis. What the case is unable to do is to publish results that are grounded in a final vocabulary. Therefore, generalisations from a research programme are not possible. What is possible are tentative ideas, concepts, and frameworks that allow dialogue and the issue of transferability applied. As noted in Chapter Seven, it is the reader who has the responsibility to determine the issue regarding transferability. Therefore the case allows for edifying philosophy, in other words, dialogue for open discussion whereby participants can utilise the concept of transferability to determine if it makes sense for them.

For example, from the case it is possible to obtain discussion points regarding the following themes:

Grand Narrative Why did the airline change its strategy?

Given the models that were available why influenced their choice?

Describe the context and what can you learn from your contextual analysis?

Internal Marketing Is this an issue for all organisations? 
Could have they handled it better?

Marketing Research Is marketing research an issue, especially if there is no evidence of a marketing plan being referred to?

Pricing Can the different pricing models be transferred to other industries?

Crusade Discuss whether every project needs a champion.

Political What was the role of the CEO?

Are there varying degrees of political necessity in organisations?

Distribution How has distribution changed in the industry? Are other industries that have similar issues?

From the point of view of praxis, discussion based on the above questions will allow for insights and understanding, rather than attempting to ground the findings into truth statements.

\section{Limitations of Research}

The exemplary case study was an attempt to illustrate the use of postmodern narrative theory. As such it was an attempt to explore strategic marketing decision making. It could be argued that three interviews were insufficient to gain what might be called 'enough data'. However, the aim was not to gain a final outcome or conclusion but to gain polyphonic voices that were counter to the grand narrative. The limiting factor was to obtain stories from those involved in the decision making. Another two participants who were involved in the decision making, one being the Chief Executive, would have gained interesting information, but they had left the industry by the time the interviews took place. 
Other stakeholders could have been interviewed, for example, cabin crew and checkin staff, but their input would be more about the implementation of the strategy than about the decision making.

It could be argued that the stories as told by the respondents were self serving, but this brings in the question of reliability. If, following Rorty, the task is not to gain foundational knowledge but to gain different perspectives then the question of reliability is of mute interest. What is of concern is that the stories have internal consistency, and they make sense in light of the grand narrative and the other participants stories. It was interesting to not that there were no contradictory claims made - just different perspectives, and this is the point of postmodern narrative theory.

Boje has claimed that it is better to gain stories from being involved in the decision making. This would involve the researcher having the role as a participant observer. Whilst this would be the ideal situation to understand praxis, it is difficult to achieve in practice, particularly as this decision involved different projects being carried out at the same time, with weekly committee updates.

\section{Reflexivity}

The notion of personal reflexivity in this research needs to be acknowledged.

Reflexivity is a term that has gained significance within post-structuralism and postmodernism, where it is maintained that subjects do not exist in any real sense and researchers are unable to study objects as independent entities. Consequently, it is 
impossible to make objective observations as any observation will be socially situated in the world of the observer and the observed. The outcome of this is the recognition that the researcher is implicated in the data as he/she is involved through the interview process and the interpretation of the data. Reflexivity is the acknowledgement of this process, that is, the relationship between the researcher and the researched.

Therefore, it needs to be acknowledged that during the period of the Air New Zealand domestic Express launch I was taking the one hour flight between Auckland and Wellington on a weekly basis so was able to observe the changes made, and on odd occasions talk to cabin crew about issues surrounding the changes. Therefore, my participation as a passenger needs to be acknowledged and that the narrative obtained in this research will be tempered by my own experience.

Also it needs to be acknowledged that Air New Zealand is the national carrier, and as such, has a special place within New Zealand culture. Therefore, when it was thought that the airline would be liquidated due to the Ansett debacle, the issue was aired in newspapers and national radio, and was a topic of conversation amongst friends and colleagues.

Also, my role as a marketing academic influenced my perspective of the research problem. This can be evidenced by the way that the grand narrative has been written. If an accounting or a human resource academic had written the case it may have taken on a different slant. 
Given the above three issues, it can be seen that I was involved in the situation and had an emotional tie to the national carrier, that is, Air New Zealand. In this respect it can be seen that the case study was not an objective observation.

Reflexivity has occurred at another level. My involvement as a marketing academic came after eight years in business and having just completed an MA in Philosophy on Heidegger's 'Being and Time'. With a good dose of Wittgenstein and Nietzsche in my Honours year, I came to the teaching of marketing with some disquiet about the status of theory within the discipline. There also seemed to be an unusual juxtaposition within the discipline whereby the majority of journal articles were based on logical empiricism or an applied scientism on the one hand, with text books utilising case studies backed up by 'exampling' to make salient points on the other hand.

It is with this background that I decided to embark on this thesis. However, the journey has been one of discovery and challenges. From wanting to nail a definitive theory of marketing and claiming that a new paradigm was the only way to go at the beginning of the process, I have now arrived in a different space, where it would seem that no grand theory is possible, nor even desirable, and at best we can understand particular situations and make sense of them at that level.

\section{Future Research}

There are a number of directions where future research would be beneficial. The first area is that of postmodern writing. Within the extant literature there has been articles about the topic of marketing, that is, descriptions of postmodernism, but little 
research undertaken with a postmodern approach. One of the difficulties is that with postmodern research one has to give up old habits, in particular the search for foundational knowledge and the impulse to attempt to derive generalisations. Within marketing the majority of academics will have been touched by postmodern thinking or images, even if it is only at a cultural level. However, the task to conduct research with a postmodern style is a challenge.

The second area of future research is to undertake studies that concentrate on praxis, that is, how marketing managers think and understand their world. Within this broad area is the task of exploring decision making in more detail. This thesis has attempted to analyse marketing decision making from a sense-making perspective, but additional work in this area would clarify the issues and develop the topic.

Finally, future research would allow what Rorty calls edifying philosophy. The important point about conducting research is not to gain foundational knowledge, but to undertake conversations and discussions where ideas are shared. In other words, being able to participate in meaningful occasions where minds are stretched and hopes kindled, with the outcome that academics become more focused on practice and practitioners become wiser. 


\section{Conclusion}

This thesis has been a narrative in itself. The story has been about the need for marketing management to adopt a new science of philosophy, based on the linguistic turn. The new philosophy of science is anti-essentialism, anti-foundational and asserts that there is no objective reality. Instead, any conception of reality is language dependent.

The thesis also explored the notion of praxis. What is of interest is how practical wisdom is used in a marketing management situation. Neo-pragmatism, coupled with narrative theory as a methodology, was put forward as an alternative philosophy of social science to examine the issue of praxis.

A number of themes arise from the adoption of neo-pragmatism. First it is based on affirmative postmodernism where reason within a situation, or language game, is used as a means to generate knowledge. Any truth statements or theory will be based on the particular situation and therefore generalisable theoretical knowledge is not possible. However, it needs to be noted that neo-pragmatism overcomes the intellectual impasse posed by some postmodern thinkers. Therefore neo-pragmatism moves beyond postmodernism by allowing reason and truth to develop in a relativist epistemological sense.

The second theme is that the art versus science debate is resolved. It is claimed that as the art or science problem is based on Cartesian dualism, it is rejected as it is based on an attempt to determine foundational knowledge. By accepting neo- 
pragmatism it can be seen that marketing is both an art and a science, but not a science in the scientistic sense.

The third theme is that of relevance. Following the work of Schön it is argued that the marketing management discipline should reject technical rationality and develop a research approach based on praxis.

Finally, by accepting the linguistic turn in modern philosophy the marketing management school can be analysed with respect to discourses being used. Therefore, it is suggested that instead of discussing issues in terms of paradigm, a more fruitful discussion would be based on the discourses that are used by marketing academics and practitioners. In this way marketing will emerge stronger - as a discursive practice. 


\section{References}

Achrol, R. S. 1991, 'Evolution of the Marketing Organization: New Forms for Turbulent Environments', Journal of Marketing, vol. 55, no. 4, pp. 77-93.

Achrol, R. S. 1997, 'Changes in the Theory of Interorganizational Relations in Marketing: Toward a Network Paradigm', Journal of the Academy of Marketing, vol. 25, no. 1, pp. 56-71.

Addis, M. \& Podesta, S. 2005, 'Long life to marketing research: a postmodern view', European Journal of Marketing, vol. 39, no. 3/4, pp. 386-412.

Addleson, M. 1996, 'Resolving the Spirit and Substance of Organizational Learning', Journal of Organizational Change Management, vol. 9, no. 1, p. 32.

Agassi, J. 2002, 'A Touch of Malice', Philosophy of Social Sciences, vol. 32, no. 1, pp. 107-119.

Allbright, D. 1999, 'Toward a logic of market-oriented organizational sensemaking', American Marketing Association, vol. 10, pp. 296-304.

Alvesson, M. \& Deetz, S. 1996, 'Critical Theory and Postmodernism Approaches to Organizational Studies' in Handbook of Organization Studies, eds S. Clegg et al, Sage Publications, London.

Alvesson, M. \& Karreman, D. 2000, 'Taking the Linguistic Turn in Organizational Research: Challenges, Responses, Consequences', The Journal of Applied Behavioral Science, vol. 36, no. 2, pp. 136-158.

Anderson, G. 1998, 'Creating Moral Space in Prenatal Genetic Services', Qualitative Health Research, vol. 8, no. 2, pp. 168-187.

Anderson, L. M. 1994, 'Marketing Science: Where's the Beef ?', Business Horizons, vol. 37, no. 1, pp. 8-16.

Anderson, P. F. 1983, 'Marketing, Scientific Progress and Scientific Method', Journal of Marketing, vol. 47, pp. 18-31.

Anderson, P. F. 1986, 'On Method in Consumer Research: A Critical Relativist Perspective', Journal of Consumer Research, vol. 13, pp. 155-173.

Ardley, B. 2006, 'Situated learning and marketing: Moving beyond the rational technical thought cage', Marketing Intelligence \& Planning, vol. 24, no. 3, pp. 202217.

Aristotle. 1970, Ethics, trans J. A. K. Thomson, Penguin Books, Harmondsworth, England.

Arndt, J. 1980, 'Perspectives for a Theory of Marketing', Journal of Business Research, vol. 8, no. 3, pp. 389-402.

Arndt, J. 1983, 'The Political Economy Paradigm: Foundation for Theory Building in Marketing', Journal of Marketing, vol. 37, pp. 44-54. 
Arndt, J. 1985, 'On Making Marketing Science More Scientific: Role of Orientations, Paradigms, Metaphors, and Puzzle Solving', Journal of Marketing, vol. 49, pp. 11-23.

Arnold, S. \& Fischer, E. 1994, 'Hermeneutics and Consumer Research', Journal of Consumer Research, vol. 21, pp. 55-70.

Arnould, E. \& Thompson, C. 2005, 'Consumer Culture Theory (CCT): Twenty Years of Research', Journal of Consumer Research, vol. 31, no. 4, pp. 868-882.

Arnould, E. \& Wallendorf, M. 1994, 'Market-Oriented Ethnography: Interpretation Building and Marketing Strategy Formulation', Journal of Marketing Research, vol. 31, pp. 484-504.

Bagozzi, R. P. 1984, 'A Prospectus for Theory Construction in Marketing', Journal of Marketing, vol. 48, pp. 11-29.

Baker, M. J. 2000, 'Marketing - Philosophy or Function?' in Marketing Theory, ed. M. Baker, Thomson Learning, London.

Baker, S. \& Holt, S. 2004, 'Making Marketers Accountable: A Failure of Marketing Education?', Marketing Intelligence and Planning, vol. 22, no. 5, pp. 557-567.

Baldridge, D., Floyd, S. \& Markoczy, L. 2004, 'Are Managers from Mars and Academicians from Venus? Toward an Understanding of the Relationship between Academic Quality and Practical Relevance', Strategic Management Journal, vol. 25, pp. 1063-1074.

Barry, D. \& Elmes, M. 1997, 'Strategy Retold: Toward a Narrative View of Strategic Discourse', Academy of Management, vol. 22, no. 2, pp 429-452.

Baudrillard, J. 1978, Critique of the Political Economy of the Sign, Telos Press, St. Louis.

Baudrillard, J. 1979, De la seduction, Galilee, Paris.

Baudrillard, J. 1983, In the Shadow of the Silent Majorities, Semiotext, New York.

Baudrillard, J. 1994, Simulacra and Simulation, University of Michigan Press, Ann Arbor.

Baumol, W. J. 1957, 'On the Role of Marketing Theory', Journal of Marketing, pp. 413-418.

Beech, N. \& McCalman, J. 1997, 'Sex, Lies and Videotropes: Narrative and Commitment in High Technology Teams', Journal of Applied Management Studies, vol. 6, no. 1, pp. 77-92.

Belk, R. 1996, 'Hyperreality and Globalization: Culture in the Age of Ronald McDonald', Journal of International Consumer Marketing, vol. 8, no. 3, pp. 23-37.

Belk, R. 2000, 'Pimps for paradise: missionaries monetary funds, and marketers', Marketing Intelligence \& Planning, vol. 18, no. 6/7, pp. 337-344.

Belk, R. 1988, 'Possessions and the Extended Self', Journal of Consumer Research, vol. 15 , pp. 139-168.

Belk, R. 1988b, 'Third World Consumer Culture;' in Marketing and Development: Towards Broader Dimensions, eds E. Kumku \& A.F. Firat, JAI, USA. 
Belk, R. 1985, 'Materialism: Trait Aspects of Living in the Material World', Journal of Consumer Research, vol. 12, pp. 265-280.

Belk, R. \& Coon, G. 1993, 'Gift Giving as Agapic Love: An Alternative to the Exchange Paradigm Based on Dating Experience', Journal of Consumer Research, vol. 20, pp. 393-417.

Belk, R. \& Pollay, R. 1985, 'Images of Ourselves: The Good Life in Twentieth Century Advertising', Journal of Consumer Research, vol. 11, pp. 887-897.

Bernstein, R. 1983, Beyond Objectivism and Relativism: Science, Hermeneutics, and Praxis, Basil Blackwell, Oxford.

Bernstein, R. 1993, 'Postmodernism, Dialogue and Democracy' in Postmodern Contentions: Epochs, Politics, Space, eds J. Jones et al, Guilford Press, New York.

Best, S. \& Kellner, D. 1991, Postmodern Theory: Critical Interrogations, Macmillan and Guildford Press, London.

Blaug, M. 1976, 'Kuhn versus Lakatos or Paradigms versus Research Programmes in the History of Economics', in Method and Appraisal in Economics, ed. S. Latsis, Cambridge University Press, Cambridge.

Boje, D. 1991, 'The Storytelling Organization: A Study of Story Performance in an Office-Supply Firm', Administrative Science Quarterly, vol. 36, no. 1, pp. 106-126.

Boje, D. 1995, 'Stories of the Storytelling Organization: A Postmodern Analysis of Disney as "Tamara-Land"', Academy of Management Journal, vol. 38, no. 4, pp. 997-1035.

Boje, D. 2001, Narrative Methods for Organizational and Communication Research, Sage Publications, London.

Boje, D., Luhman, J. T. \& Baack, D. E. 1999, 'Hegemonic Stories and Encounters Between Storytelling Organisations', Journal of Management Inquiry, vol. 8, no. 4, pp. 340-360.

Bowers, J. 1992, 'Postmodernity and the Globalisation of Technoscience: The Computer, Cognitive Science and War' in Postmodernism and the Social Sciences, eds J. Doherty et al, MacMillan, London.

Boyce, M. E. 1995, 'Collective Centring and Collective Sense-making in the Stories and Storytelling of One Organization', Organization Studies, vol. 16, no. 1, pp. 107137.

Boyce, M. E. 1996, 'Organizational Story and Storytelling: A Critical Review', Journal of Organizational Change Management, vol. 9, no. 5, p. 5.

Bradley, R. 1999, 'Review: Explorations in Economic Methodology: From Lakatos to Empirical Philosophy of Science by Roger Backhouse', British Journal of the Philosophy of Science, vol. 50, pp. 316-318.

Brennan, R. \& Ankers, P. 2004, 'In Search of Relevance: Is there an AcademicPractitioner Divide in Business-to-Business Marketing?', Marketing Intelligence and Planning, vol. 22, no. 5, pp. 511-519.

Brodie, R., Coviello, N., Brookes, R. \& Little, V. 1997, 'Towards a Paradigm Shift in Marketing? An Examination of Current Marketing Practices', Journal of Marketing Management, vol. 13, pp. 383-406. 
Brown, A. D. 1998, 'Narrative, Politics and Legitimacy in an IT Implementation', Journal of Management Studies, vol. 35, no. 1, pp. 35-58.

Brown, C. 1992, Organization Studies and Scientific Authority, Sage, London.

Brown, S. 1993, 'Postmodern Marketing?', European Journal of Marketing, vol. 27, no. 4, pp. 19-34.

Brown, S. 1994, 'Marketing as Multiplex: Screening Postmodernism', European Journal of Marketing, vol. 28, no. 8, pp. 27-51.

Brown, S. 1995, 'Life Begins at 40? Further Thoughts on Marketing's "Mid-life Crisis"', Marketing Intelligence \& Planning, vol. 13, no. 1, pp. 4-18.

Brown, S. 1995a, Postmodern Marketing, Routledge, London.

Brown, S. 1996, 'Art or Science?: Fifty Years of Marketing Debate', Journal of Marketing Management, vol. 12, pp. 243-267.

Brown, S. 1999, 'Postmodernism: The End of Marketing?' in Rethinking Marketing, eds D. Brownlie et al, Sage Publications, London.

Brown, S. 2001, 'Torment Your Customers (They'll love it)', Harvard Business Review, vol. 79, no. 9, pp. 82-88.

Brown, S. 2002, 'Who Moved my Muggle? Harry Potter and the Marketing Imaginarium', Marketing Intelligence and Planning, vol. 20, no. 3, pp. 134-148.

Brown, S. 2003, 'Crisis, What Crisis? Marketing, Midas, and the Croesus of Representation', Qualitative Market Research, vol. 6, no.3, pp. 194-205.

Brown, S. \& Schau, H. J. 2007, 'Writing consumer research: The world according to Belk', Journal of Consumer Behaviour, vol. 6, pp. 349-368.

Brownlie, D. 1997, 'Beyond Ethnography: Towards Writerly Accounts of Organizing in Marketing', European Journal of Marketing, vol. 31, no. 3/4, p. 264.

Brownlie, D. \& Saren, M. 1992, 'The Four Ps of the Marketing Concept: Prescriptive, Polemical, Permanent and Problematical', European Journal of Marketing, vol. 26, no. 4, pp. 34-47.

Brownlie, D., Saren, M., Whittingdon, R. \& Wensley R. 1994, 'The New Marketing Myopia: Critical Perspectives on Theory and Research in Marketing - Introduction', European Journal of Marketing, vol. 28, no. 3, pp. 6-12.

Brownlie, D. \& Spender, J. 1995, 'Managerial judgement in strategic marketing: some preliminary thoughts', Management Decision, vol. 33, no. 6, pp. 39-50.

Brownlie, D., Saren, M., Wensley R \& Whittingdon, R. 1999, 'Marketing Disequilibrium: On Redress and Restoration', in Rethinking Marketing, eds D. Brownlie et al, Sage Publications, London.

Burrell, G. \& Morgan, G. 1979, Sociological Paradigms and Organisational Analysis, Heinemann, London.

Burton, D. 2001, 'Critical Marketing Theory: the Blueprint?', European Journal of Marketing, vol. 35, no. 5/6, pp. 722-746.

Burton, D. 2005, 'Marketing Theory Matters', British Journal of Management, vol. $16, \mathrm{pp}$.

5-18. 
Bush, V., Harris, S. \& Bush, A. 1997, 'Establishing Ethical Boundaries for Service Providers: A Narrative Approach', The Journal of Services Marketing, vol. 11, no. 4, pp. 265-277.

Buttle, F. A. 1994, 'New Paradigm Research in Marketing', European Journal of Marketing, vol. 28, no. 9, pp. 8-11.

Buzzell, R. D. 1963, 'Is Marketing a Science', Harvard Business Review, vol. 41, pp. $32-40$.

Calas, M. B. \& Smircich, L. 1999, 'Past Postmodernism? Reflections and Tentative Directions', The Academy of Management Review, vol. 24, no. 4, pp. 649-671.

Campbell, C. 1983, 'Romanticism and the Consumer Ethic: Intimations of a Weberstyle Thesis', Sociological Analysis, vol. 44, no. 4, pp. 279-296.

Campbell, C. 1987, The Romantic Ethic and the Spirit of Modern Consumerism, Blackwell, Oxford.

Cannella, A. A. \& Paetzold, R. L. 1994, 'Pfeffer's Barriers to the Advance of Organizational Science: A Rejoinder', The Academy of Management Review, vol. 19, no. 2, pp. 331-341.

Carr, A. 2002, 'The Embrace of Hermeneutics: The Implications for Organisation Studies and Public Administration' in Proceedings of the Fifteenth Symposium on Public Administration Theory, Cleveland, Ohio.

Carson, D. \& Coviello, N. 1996, 'Qualitative Research Issues at the Marketing/ Entrepreneurship Interface', Marketing Intelligence \& Planning, vol. 14, no. 6, p. 51.

Chalmers, A. F. 1982, What is this thing called Science?, Open University Press, Milton Keynes.

Chase, S. 2005, 'Narrative Inquiry' in Handbook of Qualitative Research, eds N. Denzin \& Y. Lincoln, Sage Publications, Thousand Oaks.

Chia, R. 1995, 'From modern to postmodern organizational analysis', Organization Studies, vol. 16, no. 4, pp. 579-604.

Chia, R. 2000, 'Discourse Analysis as Organizational Analysis', Organization, vol. 7, no. 3, pp 513-518.

Chia, R. 2000a, 'Some Responses and Commentaries', Organization, vol. 7, no. 3, pp. 536-541.

Coffey, A. \& Atkinson, P. 1996, Making Sense of Qualitative Data, Sage

Publications, London.

Connell, A. F. \& Nord, W. R. 1996, 'The Bloodless Coup: The Infiltration of Organization Science by Uncertainty and Values', Journal of Applied Behavioral Science, vol. 32, no. 4, pp. 407-427.

Conrad, J. 1986, Lord Jim, Penguin Books, London.

Cooper, R. 1986, 'Organization/Disorganization', Social Science Information, vol. 25, no. 2, pp. 299-335.

Cooper, R. \& Burrell, G. 1988, 'Modernism, Postmodernism and Organizational Analysis: An Introduction', Organization Studies, vol. 9, no. 1, pp. 91-112. 
Cornelissen, J. 2002, 'Academic and Practitioner Theories of Marketing', Marketing Theory, vol. 2, no. 1, pp. 133-143.

Cova, B. \& Pace, S. 2006, 'Brand community of convenience products: new forms of customer empowerment - the case "my Nutella The Community"', European Journal of Marketing, vol. 40, no. 9/10, pp. 1087-1105.

Cova, B. \& Elliott, R., 2008, 'Everything you always wanted to know about interpretive consumer research but were afraid to ask', Qualitative Market Research, vol. 11, no. 2, pp. 121-129.

Craig-Lees, M. 2001, 'Sense Making: Trojan Horse? Pandora's Box?', Psychology and Marketing, vol. 18, no. 5, pp. 513-526.

Cunningham, A. C. 1999, 'Commentary Confessions of a Reflective Practitioner: Meeting the challenges of marketing's destruction', European Journal of Marketing, vol. 33 , no. $7 / 8$, p. 685 .

Currie, M. 1998, Postmodern Narrative Theory, Macmillan Press, London.

Czarniawska, B. 1997, 'A Four Times Told Tale: Combining Narrative and Scientific Knowledge in Organization Studies', Organization, vol. 4, no. 1, pp 7-30.

Dallmayr, F. 1993, 'Modernity in the Crossfire: Comments on the Postmodern Turn' in Postmodern Contentions: Epochs, Politics, Space, eds J. Jones et al, Guilford Press, New York.

Davies, A. \& Elliott, R. 2006, 'The Evolution of the Empowered Consumer', European Journal of Marketing, vol. 40, no. 9/10, pp. 1106-1121.

Davies, A. \& Fitchett, J. A. 2005, 'Beyond Incommensurability? Empirical Expansion on Diversity in Research', European Journal of Marketing, vol. 39, no.3/4, pp. 272-293.

Dawes, J. \& Brown R. B. 2000, 'Postmodern Marketing: Research Issues for Retail Financial Services', Qualitative Market Research, vol. 3, no. 2, p. 90.

Day, G. S. \& Montgomery, D. B. 1999, 'Charting New Directions for Marketing', Journal of Marketing, vol. 63, pp. 3-13.

De Cock, C. \& Rickards, T. 1995, 'A Rejoinder to and Reply from Weaver and Gioia', Organization Studies, vol. 16, no. 4, pp. 699-705.

Deetz, S. 1996, 'Describing Differences in Approaches to Organization Science:

Rethinking Burrell and Morgan and Their Legacy', Organization Science, vol. 7, no. 2, pp. 191-207.

Delanty, G. 1997, Social Science: Beyond Constructivism and Realism, Open Univesity Press, Buckingham.

Demirdjian, Z. S. \& Sengunder, T. 2004, 'Perspectives in Consumer Behaviour: Paradigm Shifts in Prospect', Journal of American Academy of Business, vol. 4, no. $1 / 2$, p. 348 .

Denzin, N. K. 1989, Interpretive Interactionism, Sage, Newbury Park.

Derrida, J. 1982, Margins of Philosophy, trans A. Bass, University of Chicago Press, Chicago. 
Derrida, J. 1988, 'Afterword: Toward an Ethic of Discussion', in Limited Inc, ed. G. Graff, Evanston, Illinois.

Deshpande, R. 1983, '"Paradigms Lost": On Theory and Method in Research in Marketing', Journal of Marketing, vol. 47, pp. 101-110.

DiMaggio, P. 1995, 'Comments on "What theory is not", Administrative Science Quarterly, vol. 40, no. 3, pp. 391-397.

Dixon, D. F. \& Wilkinson, I. F. 1989, 'An Alternative Paradigm for Marketing Theory', European Journal of Marketing, vol. 23, no 8, pp. 59-69.

Donaldson, L. 1997, 'A Positivist Alternative to the Structure-Action Approach', Organization Studies, vol. 18, no. 1, pp. 77-93.

Dow, S. 1992, 'Postmodernism and Economics' in Postmodernism and the Social Sciences, eds J. Doherty et al, MacMillan, London.

du Toit, A. 2003, 'Knowledge: A Sense Making Process Shared Through Narrative', Journal of Knowledge Management, vol. 7, no. 3, pp. 27-37.

Dupre, L. 1993, 'Postmodernity or Late Modernity? Ambiguities in Richard Rorty's thought', The Review of Metaphysics, vol. 47, no. 2, p. 277.

Dyer, W. G. \& Wilkins, A. L. 1991, 'Better Stories, Not Better Constructs, to Generate Better Theory: A rejoinder to Eisenhardt', Academy of Management Review, vol. 16, no. 3, pp. 613-619.

Eastman, W. N. \& Bailey, J. R. 1996, 'Epistemology, Action, and Rhetoric: Past and Present Connections', Journal of Applied Behavioural Science, vol. 32, no. 4, pp. 455-461.

Easton, G. 2002, 'Marketing: A Critical Realist Approach', Journal of Business Research, vol. 55, no. 2, p. 103.

Elman, C. \& Elman, M. F. 1997, 'Lakatos and Neorealism: A Reply to Vasquez', American Political Science Review, vol. 91, no. 4, pp. 923-926.

Erickson, G. W. 1991, 'Our Man Rorty', Chicago Review, vol. 38, no. 3, pp. 94-104.

Fawundu, F. 1991, 'Blaug on Kuhn versus Lakatos and the Marginalist Revolution', Atlantic Economic Journal, vol. 19, no. 1, pp. 29-32.

Feyerabend, P. 1988, Against Method, revised edition, Verso, London.

Firat, A. F., Dholakia, N. \& Venkatesh, A. 1995, 'Marketing in a Postmodern World', European Journal of Marketing, vol. 29, no. 1, pp. 40-56.

Firat, A. F. \& Venkatesh, A. 1995, 'Liberatory Postmodernism and the

Reenchantment of Consumption', Journal of Consumer Research, vol. 22, pp. 239267.

Fisher, W. R. 1985, 'The Narrative Paradigm: An elaboration', Communication Monographs, vol. 52, pp. 347-367.

Fletcher, G. J. O. 1996, 'Realism versus Relativism in Psychology', American Journal of Psychology, vol. 109, no. 3, pp. 409-429.

Flew, A. 1971, An Introduction to Western Philosophy, Thames \& Hudson, London.

Foucault, M. 1982, The History of Sexuality, vol. 1 An Introduction, trans R. Hurley, Vintage Books, New York. 
Foucault, M. 1967 [1966], Madness and Civilisation: A History of Insanity in the Age of Reason, trans R. Howard, Routledge, London.

Foucault, M. 1972 [1966], The Order of Things: An Archaeology of the Human Sciences, trans A. Sheridan, Routledge, London.

Foucault, M. 1974 [1969], The Archaeology of Knowledge, trans A. Sheridan, Routledge, London.

Fox, C. J. \& Miller, H. T. 1995, Postmodern Public Administration, Sage Publications, Thousand Oaks.

Franke, N. \& Mazanec, J. 2005, 'The six identities of marketing: a vector quantization of research approaches', European Journal of Marketing, vol. 40, no. 5/6, pp. 634-661.

Fulford, R. 1999, The Triumph of Narrative, Broadway Books, New York.

Gadamer, H. 1975, Truth and Method, eds G. Barden \& J. Cumming, Seabury Press, New York.

Gee, J. P. 1999, An Introduction to Discourse Analysis, Routledge, London.

Gergen, K. 1992, 'Organization Theory in the Postmodern Era' in Rethinking Organizations: New Directions in Organization Theory and Analysis, eds M. Reed \& M. Hughes, Sage Publications, London.

Gergen, K. 2001, 'Self-Narration in Social Life' in Discourse Theory and Practice: A Reader, eds M. Wetherall et al, Sage Publications, London.

Gibson, H., Tynan, C. \& Pitt, L. 1993, 'What is marketing?: a qualitative and quantitative analysis of marketing decisions', Proceedings, Marketing Education Group Conference, Loughborough.

Giddens, A. 1979, Central problems in social theory : action, structure, and contradiction in social analysis, Macmillan, London.

Gilmore, A. 1997, 'Understanding marketing management competencies within a service context', Irish Marketing Review, vol. 10, no. 1, pp. 36-46.

Goulding, C. 1999, 'Consumer Research, Interpretive Paradigms and Methodological Ambiguities', European Journal of Marketing, vol. 33, no. 9/10, p. 859.

Goulding, C. 2003, 'Issues in representing the postmodern consumer', Qualitative Market Research, vol. 6, no. 3, pp. 152-159.

Goulding, C. 2005, 'Grounded theory, ethnography and phenomenology: A comparative analysis of three qualitative strategies for marketing research', European Journal of Marketing, vol. 39, no. 3/4, pp. 294-308.

Graham, E. \& Doherty, J. 1992, 'Postmodern Horizons' in Postmodernism and the Social Sciences, eds J. Doherty et al, MacMillan, London.

Graham, E., Doherty, J. \& Malek, M. 1992, 'Introduction: The Context and Language of Postmodernism' in Postmodernism and the Social Sciences, eds J. Doherty et al, MacMillan, London.

Grant, P. \& Perrin, L. 2002, 'Small Business and Entrepreneurial Research: Metatheories, paradigms and prejudices', International Small Business Journal, vol. 20, no. 2, pp. 185-211. 
Greenley, G., Hooley, G. \& Saunders, J. 2004, 'Management processes in marketing planning', European Journal of Marketing, vol. 38, no. 8, pp. 933-955.

Greenwood, D. \& Levin, M. 2005, 'Reform Through Action Research' in Handbook of Qualitative Research, eds N. Denzin \& Y. Lincoln, Sage Publications, Thousand Oaks.

Grether, E. T. 1965, 'An Emerging Apologetic of Managerialism?: Theory In Marketing', Journal of Marketing Research, vol. 11, pp. 190-195.

Gronroos, C. 1990, 'Marketing Redefined', Management Decision, vol. 28, no. 8, pp. 5-9.

Gronroos, C. 1994, 'From Marketing Mix to Relationship Marketing: Towards a Paradigm Shift in Marketing', Management Decision, vol. 32, no. 2, pp. 4-20.

Guliz, G. \& Welk, R. 1996, 'I'd like to buy the world a Coke: Consumptionscapes of the "Less Affluent World"', Journal of Consumer Policy, vol. 19, no. 3, pp. 271-304.

Gummesson, E. 1997, 'Relationship Marketing as a Paradigm Shift: some conclusions from the 30R approach', Management Decision, vol. 35, no. 4, p. 267.

Gummesson, E. 2001, 'Are Current Research Approaches in Marketing Leading us Astray?, Marketing Theory, vol. 1, no. 1, pp. 27-48.

Gummesson, E. 2003, 'All Research is Interpretive!', The Journal of Business and Industrial Marketing, vol. 18, no. 6/7, p. 482.

Habermas, J. 1977, 'A Review of Gadamer's Truth and Method' in Understanding and Social Inquiry, eds F. Dallmayr \& T. McCarthy, University of Notre Dame Press, Paris.

Hackley, C. E. 1998, 'Philosophy, Science, Marketing Theory and Action Men with Real Hair', Academy of Marketing Annual Conference, Manchester Metropolitan University, Manchester.

Hackley, C. E. 1999, 'Tacit Knowledge and the Epistemology of Expertise in Strategic Marketing Management', European Journal of Marketing, vol. 33, no.7/8, pp. 720-732.

Hackley, C.E. 2001, 'Towards a Post-Structuralist Marketing Pedagogy - or from Irony to Despair', European Journal of Marketing, vol. 35, no. 11/12, p. 1184.

Haldane, J. 1992, 'Cultural Theory, Philosophy and the Study of Human Affairs: Hot Heads and Cold Feet' in Postmodernism and the Social Sciences, eds J. Doherty et al, MacMillan, London.

Harre, R. 1971, 'Joynson's Dilemma', Bull. Br. Pscyol. Soc, vol. 24, pp. 115-119.

Harvey, D. 1995, The Condition of Postmodernity, 2nd edn, Blakewell Publishers, Massachusetts.

Harvey, M. \& Evans, M. 2001, 'Decoding Competitive Propositions: A semiotic alternative to traditional advertising research', International Journal of Market Research, vol. 43, no. 2, pp. 171-188.

Hassard, J. 1991, 'Multiple Paradigms and Organizational Analysis: A Case Study', Organization Studies, vol. 12, no. 2, pp. 275-299. 
Hassard, J. 1994, 'Postmodern Organizational Analysis: Toward a Conceptual Framework', Journal of Management Studies, vol. 31, no. 3, pp. 303-324.

Hatch, M. \& Rubin, J. 2006, 'The hermeneutics of branding', Brand Management, vol. 14 , no. $1 / 2$, pp. 40-59.

Heidegger, M. 1983, Being and Time, trans J. Macquarrie \& E. Robinson, Basil Blackwell, Oxford.

Hintikka, J. 1988, 'On the Incommensurability of Theories', Philosophy of Science, vol. 55, pp. 25-38.

Hirschman, E. 1985, 'Scientific Style and the Conduct of Consumer Research', Journal of Consumer Research, vol. 12, pp. 225-239.

Hirschman, E. 1993, 'Ideology in Consumer Research, 1980 and 1990: a Marxist and Feminist Critique', Journal of Consumer Research, vol. 19, pp. 537-555.

Hochschild, A. R. 1983, The Managed Heart : Commercialization of Human Feeling, University of California Press, Berkeley.

Hogg, M. K. \& Maclaran, P. 2008, 'Rhetorical issues in writing interpretivist consumer research', Qualitative Market Research: An International Journal, vol. 11, no. 2, pp. 130-146.

Holbrook, M. B. 1985, 'Why Business is Bad for Consumer Research' in Advances in Consumer Research, eds E. Hirschman \& M. Holbrook, Association for Consumer Research, Ann Arbor.

Hollinger, R. 1994, Postmodernism and the Social Sciences, Sage Publications, California.

Hollis, M. 1994, The Philosophy of Science, Cambridge University Press, Cambridge.

Hopkinson, G. C. 2001, 'Influence in Marketing Channels: A Sense-Making Investigation', Psychology and Marketing, vol. 18, no. 5, pp. 423-444.

Horne, J. S. 1993, 'Rorty's Circumvention of Argument: Redescribing Rhetoric', The Southern Communication Journal, vol. 58, no.3, pp. 169-181.

Houston, F. S. 1986, 'The Marketing Concept: What it is and What it is not', Journal of Marketing, vol. 50, no. 2, pp 81-88.

Howard, D., Savins, D., Howell, W. \& Ryans, J. 1991, 'The Evolution of Marketing Theory in the United States and Europe', European Journal of Marketing, vol. 25, no. 2, pp. 7-16.

Hudson, L. A. \& Ozanne, J. L. 1988, 'Alternative Ways of Seeking Knowledge in Consumer Research', Journal of Consumer Research, vol. 14, pp. 508-521.

Hulbert, J. M. \& Pitt, L. 1996, 'Exit Left Center Stage? The Future of Functional Marketing', European Management Journal, vol. 14, no. 1, pp. 47-60.

Hummel, R. P. 1991, 'Stories Managers Tell: Why They Are as Valid as Science', Public Administration Review, vol. 51, no. 1, p. 31.

Hunt, S. D. 1983. Marketing Theory: Conceptual Foundations of Research in Marketing, Irwin, Homewood. 
Hunt, S. D. 1990, 'Truth in Marketing Theory and Research', Journal of Marketing, vol. 54, no. 3, pp. 1-24.

Hunt, S. D. 1991, 'Positivism and Paradigm Dominance in Consumer Research: Toward a Critical Pluralism and Rapprochement', Journal of Consumer Research, vol. 18 , no. 1, pp. 32-45.

Hunt, S. D. 1992, 'For Reason and Realism in Marketing', Journal of Marketing, vol. 56, pp. 89-102.

Hunt, S. D. 1994, 'On Rethinking Marketing: Our Discipline, Our Practice, Our Methods', European Journal of Marketing, vol. 28, no. 3, pp. 13-25.

Hutchinson, T. W. 1976, On the History and Philosophy of Science and Economics, Cambridge University Press, Cambridge.

Jackson, N. \& Carter, P. 1991, 'In Defence of Paradigm Incommensurability', Organization Studies, vol. 12, no. 1, pp. 109-127.

Jackson, N. \& Carter, P. 1993, 'Paradigm Wars': A Response to Hugh Willmott', Organization Studies, vol. 14, no. 5, pp. 721-725.

Jackson, W. A. 1995, 'Naturalism in Economics', Journal of Economic Issues, vol. 29, no. 3, pp. 761-780.

Jacoby, J. 1985, 'Serving Two Masters: Perspectives on Consulting' in Advances in Consumer Research, eds E. Hirschman \& M. Holbrook, Association for Consumer Research, Ann Arbor.

Jameson, F. 1984, 'Postmodernism, or the Cultural Logic of Late Capitalism', New Left Review, vol. 146, pp. 53-92.

Jameson, F. 1988, The Ideologies of Theory: Essays 1971-1986, University of Minnesota Press, Minneapolis.

Jones, D. G. B. \& Monieson, D. D. 1990, 'Early Development of the Philosophy of Marketing Thought', Journal of Marketing, vol. 54, pp. 102-113.

Jones, J. P., Natter, W. \& Schatzki, T. R. 1993, '"Post"-ing Modernity' in Postmodern Contentions: Epochs, Politics, Space, eds J. Jones et al, Guilford Press, New York.

Kasabov, E. 2004, 'Power and Disciplining: Bringing Foucault to Marketing', Irish Marketing Review, vol. 17, no. 1/2, pp. 3-12.

Katsikeas, C. S., Robson, M. J. \& Hulbert, J. M. 2004, 'In Search of Relevance and Rigour for Research in Marketing', Marketing Intelligence and Planning, vol. 22, no. 5, pp. 568-578.

Kavanagh, D. 1994, 'Hunt versus Anderson: Round 16', European Journal of Marketing, vol. 28, no. 3, pp. 26-41.

Kennedy, K., Goolsby, J. \& Arnould, E. 2003, 'Implementing a Customer Orientation: Extension of Theory and Application', Journal of Marketing, vol. 67, pp. 67-81.

Kilduff, M. \& Mehra, A. 1997, 'Postmodernism and Organizational Research', Academy of Management, vol. 22, no. 2, pp. 453-481. 
Kincaid, H. 1996, Philosophical Foundations of the Social Sciences, Cambridge University Press, Cambridge.

Klein, N. 2000, No logo : no space, no choice, no jobs, Flamingo, London.

Knights, D. 1992, 'Changing Spaces: The Disruptive Impact of a New Epistemological Location for the Study of Management', The Academy of Management Review, vol. 17, no. 3, pp. 514-536.

Knights, D. 1997, 'Organization Theory in the Age of Deconstruction: Dualism, Gender and Postmodernism Revisited', Organization Studies, vol. 18, no. 1, pp. 120.

Kotler, P. 1975, Marketing for Nonprofit Organizations, Prentice Hall, New York.

Kotler, P. \& Keller, K. 2006, Marketing Management, Pearson Prentice Hall, New Jersey.

Krishna, A. \& Shoemaker, R.W. 1992, 'Estimating the Effects of Higher Coupon Face Values on the Timing of Redemptions, the Mix of Coupon Redeemers, and Purchase Quality', Psychology and Marketing, vol. 9, no. 6, pp. 453-467.

Kuhn, T. S. 1962, The Structure of Scientific Revolutions, University of Chicago Press, Chicago.

Kuhn, T. S. 1970, Reflections on my Critics, Cambridge University Press, Cambridge.

Lacity, M. C. \& Janson, M. A. 1994, 'Understanding Qualitative Data: A Framework of Text Analysis Methods', Journal of Management Information Systems, vol. 11, no. 2, pp. 137-156.

Laczniak, G. R. \& Michie, D. A. 1979, 'The Social Disorder of the Broadened Concept of Marketing', Journal of Academy of Marketing Science, vol. 7, no. 3, pp. 214-232.

Lakatos, I. 1970, Falsification and the Methodology of Scientific Research Programmes, Cambridge University Press, Cambridge.

Lash, S. 1992, 'Postmodernism or Modernism?: Social Theory Revisited' in Postmodern Contentions: Epochs, Politics, Space, eds J. Jones et al, MacMillan, London.

Lawson, H. 1989, 'Dismantling Truth' in Dismantling Truth: Reality in the Postmodern World, eds H. Lawson \& L. Appignanesi, Weidenfeld \& Nicolson, London.

Leone, R. P. \& Schultz R. L. 1980, 'A Study of Marketing Generalisations', Journal of Marketing, vol. 44, no. 1, pp. 23-37.

Leong, S. M. 1985, 'Metatheory and Metamethodology in Marketing: A Lakatosian Reconstruction', Journal of Marketing, vol. 49, pp. 23-40.

Leong, S. M., Sheth, J. N. \& Tan, C. T. 1994, 'An Empirical Study of the Scientific Styles of Marketing Academics', European Journal of Marketing, vol. 28, no. 8, pp. $12-26$.

Levy, S. J. 2002, 'Revisiting the Marketing Domain', European Journal of Marketing, vol. 36, no. 3, pp. 299-304. 
Lilien, G., Rangaswamy, A. \& Matanovich, T. 1998, 'The Age of Marketing Engineering', Marketing Management, vol. 7, no. 1, pp. 48-50.

Lincoln, Y. \& Guba, E. 1985, Naturalistic Inquiry, Sage Publications, Beverly Hills.

Lindblom, C. E. 1990, Inquiry and Change : The Troubled Attempt to Understand and Shape Society, Yale University Press, New Haven.

Lindblom, C. E. \& Cohen, D. K. 1979, Usable Knowledge : Social Science and Social Problem Solving, Yale University Press, New Haven.

Liswood, L. A. 1987, 'Once You've Got 'Em, Never Let 'Em Go', Sales \& Marketing Management, vol. 139, pp. 73-76.

Littler, D. \& Tynan, C. 2005, 'Where are We and Where are We Going?', European Journal of Marketing, vol. 39, no. 3/4, pp. 261-271.

Lockley, L. C. 1964, 'An Approach to Marketing Theory' in Theory in Marketing, $2^{\text {nd }}$ edn, eds R. Cox et al, Irwin, Homewood.

Lovitt, M. R. 1997, 'The New Pragmatism: Going Beyond Shewart and Deming', Quality Progress, vol. 30, no. 4, pp. 99-105.

Lowe, S., Carr, A. N. \& Thomas, M. 2004, 'Paradigmapping Marketing Theory', European Journal of Marketing, vol. 38, no. 9/10, p. 1057.

Lucey, B. M. 2000, 'Friday the 13th and the Philosophical Basis of Financial Economics', Journal of Economics and Finance, vol. 24, no. 3, pp. 294-301.

Lutz, F. 1993, 'Social Contexts of Postmodern Cultural Analysis' in Postmodern Contentions: Epochs, Politics, Space, eds J. Jones et al, Guilford Press, New York.

Machan, T. 1996, 'Indefatigable Alchemist: Richard Rorty's Radical Pragmatism', The American Scholar, vol. 65, no. 3, pp. 417-424.

Magala, S. 1997, 'The Making and Unmaking of Sense', Organization Studies, vol. 18. p. 317.

Magrath, A. J. 1986, 'When Marketing Services, 4 Ps Are Not Enough', Business Horizons, vol. 2, pp. 44-50.

Maher, P. 1990, 'Why Scientists Gather Evidence', British Journal of Philosophy of Science, vol. 41, pp. 103-119.

Marsden, R. 1993, 'The Politics of Organizational Analysis', Organization Studies, vol. 14 , no. 1 , pp. 93-124.

Mathews, K. M., Whilte, M. C. \& Long, R. G. 1999, 'The Problem of Prediction and Control in Theoretical Diversity and the Promise of the Complexity Sciences', Journal of Management Inquiry, vol. 8, no. 1, pp. 17-31.

Mayer, R., Job, K. \& Ellis, N. 2000, 'Ascending Separate Stairways to Marketing Heaven (or careful with that axiom, Eugene!)', Marketing Intelligence and Planning, vol. 18 , no. $6 / 7$, pp. 388-399.

McCole, P. 2004, 'Refocusing Marketing to Reflect Practice: The changing role of marketing for business', Marketing Intelligence \& Planning, vol. 22, no. 5, pp. 531539.

McGowan, J. 1991, Postmodernism and its Critics, Cornell University Press, Ithaca. 
McIntyre. S. H. \& Sutherland, M. 2002, 'A Critical Analysis into the Accumulation of Marketing Knowledge at the Level of the Firm', Marketing Theory, vol. 2, no. 4, pp. 403-418.

McKenna, R. 1991, 'Marketing is Everything', Harvard Business Review, vol. 69, pp. 65-79.

McLuhan, M. 1994, Understanding Media, MIT Press, Cambridge, MA.

Miller, H. T. \& King, C. H. 1998, 'Practical Theory', American Review of Practical Administration, vol. 28, no. 1, pp. 43-60.

Mitroff, I. 1985, 'Mindshift: The Mental Revolution We are In', Technological Forecasting and Social Change, vol. 28, pp. 3-12.

Mitroll, I. 1972, 'The Myth of Objectivity or Why Science Needs a New Psychology of Science', Management Science, vol. 18, pp. 613-618.

Moberg, D. W. 1979, ‘Are There Rival, Incommensurable Theories?', Philosophy of Science, vol. 46, pp. 244-262.

Morgan, G. 1980, 'Paradigms, Metaphors, and Puzzle Solving in Organization Theory', Administrative Science Quarterly, vol. 25, pp. 605-622.

Morgan, G. 1983, Beyond Method: Strategies for Social Research, Sage Publications, California.

Morgan, G. 1986, Images of Organization, Sage, Beverley Hills, CA.

Morgan, R. M. \& Hunt, S. D. 1994, 'The Commitment-Trust Theory of Relationship Marketing', Journal of Marketing, vol. 58, pp. 20-38.

Motiram, S. \& Vakulabharanam, V. 2007, 'Corporate and Cooperative Solutions for the Agrarian Crisis in Developing Countries', Review of Radical Political Economics, vol. 39, no. 3, p 360.

Mouzelis, N. 2000, 'The Subjectivist-Objectivist Divide: Against Transcendence', Sociology, vol. 34, no. 4, pp. 741-762.

Muncy, J. A. \& Fisk, R. P. 1987, 'Cognitive Relativism and the Practice of Marketing Science’, Journal of Marketing, vol. 51, no. 1, pp. 20-33.

Murray, J., Evers, D. \& Janda, S. 1995, 'Marketing, Theory Borrowing; and Critical Reflection', Journal of Macromarketing, vol. 15, no. 2, pp. 92-106.

Murray, J., O’Driscoll, A. \& Torres, A. 2002, 'Discovering diversity in marketing practice', European Journal of Marketing, vol. 36, no. 3, pp. 373-390.

Musgrave, A. 1978, 'Evidential Support, Falsification, Heuristics, and Anarchism', in Criticism and Growth of Knowledge eds I. Lakatos \& A. Musgrave, Cambridge University Press, Cambridge.

Myrsiades, L. S. 1987, 'Corporate Stories as Cultural Communications in the Organizational Setting', Management Communication Quarterly, vol. 1, no. 1, pp. 84-120.

Natter, W. 1993, 'Signposts Towards a Poststructuralist Geography' in Postmodern Contentions: Epochs, Politics, Space, eds J. Jones et al, Guilford Press, New York.

Nilson, T. H. 1992, Value-added Marketing: Marketing Management for Superior Results, McGraw-Hill, Maidenhead. 
Novatorov, E. V. \& Crompton, J. L. 2001, 'A Revised Conceptualization of Marketing in the Context of Public Leisure Services', Journal of Leisure Research, vol. 33, no. 2, pp. 160-185.

November, P. 2004, 'Seven Reasons Why Marketing Practitioners Should Ignore Marketing Academic Research', Australasian Marketing Journal, vol. 12, no. 2, pp. 39-50.

Nuyen, A. 1992, 'Rorty's hermeneutics and the problem of relativism', Man and World, vol. 25, pp. 69-78.

O'Connor, E. S. 1997, 'Discourse at our Disposal: Stories In and Around the Garbage Can', Management Communication Quarterly, vol. 10, no.4, pp. 395-432.

O'Driscoll, A. \& Murray, J. A. 1998, 'The Academy-Marketplace Interface: Who is Leading whom and Does it Really Matter?', Irish Marketing Review, vol. 11, no. 1, pp 5-18.

O'Leary, Z. 2004, The Essential Guide to Doing Research, Sage Publications, London.

O’Shaughnessy, J. \& Holbrook, M. 1988, 'Understanding Consumer Behaviour: The Linguistic Turn in Marketing Research', Journal of the Market Research Society, vol. 30, no. 2, pp. 197-223.

O'Shaughnessy, J. \& O'Shaughnessy, N. 2002, 'Postmodernism and Marketing: Separating the Wheat from the Chaff', Journal of Macromarketing, vol. 22, no. 1, pp. 109-135.

O'Shaughnessy, J. \& O'Shaughnessy, N. 2002a, 'Ways of Knowing and Their Applicability', Marketing Theory, vol. 2, no. 2, pp. 147-164.

Ottesen, G. G. \& Gronhaug, K. 2004, 'Barriers to Practical Use of Academic Marketing Knowledge', Marketing Intelligence \& Planning, vol. 22, no. 5, pp. 520530.

Padgett, D. \& Allen, D. 1997, 'Communicating Experiences: A Narrative Approach to Creating Service Brand Image', Journal of Advertising, vol. 26, no. 4, pp. 49-62.

Parker, I. 1992, 'Discourse Discourse: Social Psychology and Postmodernity' in Postmodernism and the Social Sciences, eds J. Doherty et al, MacMillan, London.

Parker, M. 1992, 'Post-Modern Organizations or Postmodern Organization Theory', Organization Studies, vol. 13, no. 1, pp. 1-17.

Parker, M. 2000, “The Less Important Sideshow': The Limits of Epistemology in Organizational Analysis', Organization, vol. 7, no. 3, pp. 519-523.

Parker, M. \& McHugh, G. 1991, 'Five Texts in Search of an Author: A Response to John Hassard's Multiple Paradigms and Organizational Analysis', Organization Studies, vol. 12, no. 3, pp. 451-456.

Patton, M. 2002, Qualitative Research and Education Methods, $3^{\text {rd }}$ edn, Sage Publications, London.

Payne, A. 1995, Advances in Relationship Marketing, Kogan Page, London.

Pentland B. T. 1999, 'Building Process Theory with Narrative: From Description to Explanation', Academy of Management Review, vol. 24, no. 4, pp. 711-724. 
Perry, C., Riege, A. \& Brown, L. 1999, 'Realism's Role Among Scientific Paradigms in Marketing Research', Irish Marketing Review, vol. 2, no.2, pp. 16-23.

Peter, J. P. 1982, 'Realism or Relativism for Marketing Theory and Research: A Comment on Hunt's "Scientific Realism"', Journal of Marketing, vol. 56, pp. 72-79.

Peter, J. P. \& Olson, J. C. 1983, 'Is Science Marketing?', Journal of Marketing, vol. 47, pp. 111-125.

Pfeffer, J. 1993, 'Barriers to the Advance of Organizational Science: Paradigm Development as a Dependent Variable', Academy of Management Review, vol. 18, no. 4, pp. 599-620.

Phillips, D. C. 1987, Philosophy, Science, and Social Inquiry, Pergamon Press, New York.

Phillips, N. 1995, 'Telling Organizational Tales: on the role of narrative fiction in the study of organizations', Organization Studies, vol. 16, no. 4, p. 625-649.

Phillips, N. \& Brown, J. L. 1993, ‘Analyzing Communication in and around Organizations: A Critical Hermeneutic Approach', Academy of Management Journal, vol. 36, no. 6, p. 1547.

Piercy, N. F. 1998, 'Marketing Implementation: The implications of Marketing Paradigm Weakness for the Strategy Execution Process', Journal of the Academy of Marketing Science, vol. 26, no. 3, pp. 222-236.

Piercy, N. F. 2002, 'Research in Marketing: teasing with trivia or risking relevance?', European Journal of Marketing, vol. 36, no. 3, pp. 350-363.

Pirsig, R. M. 1976, Zen and the Art of Motorcycle Maintenance : an inquiry into values, Corgi Books, London.

Pitt, L. F. \& Morris, M. H. 1995, 'When Marketing and Strategy Become One', Journal of General Management, vol. 21, no. 2, pp. 21-30.

Popper, K. 1959, The Logic of Scientific Discovery, Hutchinson, London.

Poropat, A. 2002, 'Apples and Oranges: Incommensurable Paradigms and Their Implications for Teaching Organisational Development', Organization Development Journal, vol. 20, no. 3, pp. 74-86.

Price, L. \& Arnould, E. 1999, 'Commercial Friendships: Service Provider-Client Relationships in Context', Journal of Marketing, vol. 63, no. 4, pp. 38-56.

Putnam, H. 1997, 'A Half Century of Philosophy, Viewed from Within', Daedalus, vol. 126 , no. 1 , pp. 175-208.

Quine, W.V. 1969, ‘Two Dogmas of Empiricism' in Necessary Truth, eds L. Sumner and J. Woods, Random House, New York.

Ragg, E. P. 2002, 'Worlds or Words Apart? The Consequences of Pragmatism for Literary Studies', Philosophy and Literature, vol. 26, no.2, pp. 369-396.

Razzaque, M. A. 1998, 'Scientific Method, Marketing Theory Development and Academic vs Practitioner Orientation: A Review', Journal of Marketing Theory \& Practice, vol. 6, no. 1, pp. 1-15.

Reichheld, F. F. 1996, 'Learning from Customer Defections', Harvard Business Review, vol. 74, no. 2, pp. 56-69. 
Rengger, N. \& Hoffman, M. 1992, 'Modernity, Postmodernism and International Relations' in Postmodernism and the Social Sciences, eds J. Doherty et al, MacMillan, London.

Rescher, N. 1987, Scientific Realism, Reidel Publishing, Dordrecht.

Richardson, B. 2000, 'Recent Concepts of Narrative and the Narratives of Narrative Theory', Style, vol. 34, no. 2, pp. 168-175.

Ritzer, G. 1996, The McDonaldization of Society : An Investigation into the Changing Character of Contemporary Social Life, Pine Forge Press, Thousand Oaks.

Robbins, B. 1992, 'Death and Vocation: Narrativizing Narrative Theory, Publications of the Modern Language Association of America, vol. 107, no. 1, pp. $38-50$.

Rorty, R. 1979, Philosophy and the Mirror of Nature, Princeton University Press, New Jersey.

Rorty, R. 1982, Consequences of Pragmatism, University of Minnesota Press, Minneapolis.

Rorty, R. 1989, Contingency, Irony, and Solidarity, Cambridge University Press, Cambridge.

Rorty, R. 1989a, 'Science as Solidarity' in Dismantling Truth: Reality in the Postmodern World, eds H. Lawson \& L. Appignanesi, Weidenfeld \& Nicolson, London.

Rosenau, P. M. 1992, Post-Modernism and the Social Sciences, Princeton University Press, New Jersey.

Ross, W., Moore, M. \& Staelin, R. 2000, 'Recurrent Marketing Decision: Decision Complexity, Decision Focus, and Firm Performance', Marketing Letters, vol. 11, no. 4, p. 283.

Rossiter, J. 2001, 'What is marketing knowledge? Stage I: Forms of marketing knowledge', Marketing Theory, vol. 1, no. 1, pp. 9-26.

Rouleau, L. \& Clegg, S. R. 1992, 'Postmodernism and Postmodernity in Organization Analysis', Journal of Organizational Change Management, vol. 5, no. 1 , pp. 8-25.

Ruben, D. 1989, Realism in the Social Sciences in Dismantling Truth: Reality in the Postmodern World, eds H. Lawson \& L. Appignanesi, Weidenfeld \& Nicolson, London.

Saren, M. 2000, 'Marketing Theory' in Marketing Theory, 2nd edn, ed. M. Baker, Thomson Learning, London.

Saren, M. 2007, 'Marketing is everything: the view from the street', Marketing Intelligence and Planning, vol. 25, no. 1, pp. 11-16.

Sarup, M. 1993, An Introductory Guide to Post-Structuralism and Postmodernism, Harvester Wheatsheaf, Hemel Hempstead.

Saunders, J. \& Lee, N. 2005, 'Whither Research in Marketing?', European Journal of Marketing, vol. 39, no. 3/4, pp. 245-260. 
Schatzki, T. R. 1993, 'Theory at Bay: Foucault, Lyotard, and Politics of the Local' in Postmodern Contentions: Epochs, Politics, Space, eds J. Jones et al, Guilford Press, New York.

Scheffler, I. 1967, Science and Subjectivity, Bobbs-Merritt, Indianapolis.

Scherer, A. G. \& Steinmann, H. 1999, 'Some Remarks on the Problem of Incommensurability in Organisation Studies', Organisation Studies, vol. 20, no. 3, pp. 519-544.

Schipper, F. 1999, 'Phenomenology and the Reflective Practitioner', Management Learning, vol. 30, no. 4, pp. 473-485.

Schmitt, B. 1999, Experiential Marketing: How to get Customers to Sense, Feel, Think, Act, and Relate to your Company and Brands, Free Press, New York.

Schön, D.A. 1983, The Reflective Practitioner. How Professionals Think in Action, Basic Books Inc., New York.

Schouten, J. \& McAlexander, J. 1995, 'Subcultures of consumption: An ethnography of the new biker', Journal of Consumer Research, vol. 22, no. 1, pp. 43-61.

Schultz, M. \& Hatch, M. J. 1996, 'Living with Multiple Paradigms: The Case of Paradigm Interplay in Organizational Culture Studies', Academy of Management Review, vol. 21, no. 2, pp. 529-557.

Schwartzman, H. B. 1984, 'Stories at Work: Play in an Organizational Context' in Text, Play and Story, ed. E. Bruner, American Ethnological Society, Washington, DC.

Schweitzer, D. 1991, 'Marxist theories of Alientation and Reification: The Response to Capitalism, State Socialism and the Advent of Postmodernity', The International Journal of Sociology and Social Policy, vol. 11, no. 6-8, p. 27.

Shankar, A. \& Goulding, C. 2001, 'Interpretive Consumer Research: two more contributions to theory and practice', Qualitative Market Research, vol. 4, no. 1, p.7.

Shankar, A., Elliott, R. \& Goulding, C. 2001, 'Understanding Consumption: Contributions from a Narrative Perspective', Journal of Marketing Management, vol. 17, pp. 429-453.

Shankar, A. \& Patterson, M. 2001, 'Interpreting the Past, Writing the Future', Journal of Marketing Management, vol. 17, pp. 481-501.

Sheth, J. N. \& Gardner, D. M. 1982, 'History of Marketing Thought: An Update', American Marketing Association, pp. 52-58.

Shields, R. 1992, 'Social Science and Postmodern Spatialisations: Jameson's Aesthetic of Cognitive Mapping' in Postmodernism and the Social Sciences, eds J. Doherty et al, MacMillan, London.

Siegel, H. 1988, 'Relativism for Consumer Research? Comments on Anderson', Journal of Consumer Research, vol. 15, no. 1, pp. 129-132.

Simmons, G. 2008, 'Marketing to postmodern consumers: introducing the internet chameleon', European Journal of Marketing, vol. 42, no. 3/4, pp. 299-310.

Skipper, R. \& Hyman, M. R. 1990, 'Marketing and Logical Deduction', Journal of Marketing, vol. 54, pp. 89-92. 
Slevin, E. \& Sines, D. 1999, 'Enhancing the truthfulness, consistency and transferability of a qualitative study: utilising a manifold of approaches', Nurse Researcher, vol. 7, no. 2, pp. 79-97.

Small, R. G. 1993, 'Consumption and Significance: Everyday Life in a Brand-new Second-hand Bow Tie', European Journal of Marketing, vol. 27, no. 8, pp. 38-45.

Snow, C. C. 1997, 'Twenty-First-Century Organizations: Implications for a New Marketing Paradigm', Journal of the Academy of Marketing Science, vol. 25, no. 1, pp. $72-74$

Steffy, B. D. \& Grimes, A. J. 1986, 'A Critical Theory of Organization Science', Academy of Management Review, vol. 11, no. 2, pp. 322-336.

Stern, B. 1989, 'Literary Criticism and Consumer Research: Overview and Illustrative Analysis', Journal of Consumer Research, vol. 16, pp. 322-334.

Stern, B., Thompson, C. \& Arnould, E. 1998, 'Narrative Analysis of a Marketing Relationship: The Consumer's Perspective', Psychology \& Marketing, vol. 15, no. 3 , pp. 195-214.

Sutton, R. \& Staw, B. 1995, 'What theory is not', Administrative Science Quarterly, vol. 40, no. 3, pp. 371-384.

Szmigin, I. \& Foxall, G. 2000, 'Interpretive Consumer Research: how far have we come?', Qualitative Market Research, vol. 3, no. 4, pp. 187-199.

Tapp, A. 2004, 'A Call to Arms for Applied Marketing Academics', Marketing Intelligence and Planning, vol. 22, no. 5, pp. 579-590.

Tarascio, V. J. 1997, 'The Problem of Knowledge in Economics', Southern Economic Journal, vol. 64, no. 1, pp. 1-18.

Thomas, M. J. 1994, 'Marketing - in Chaos or Transition?', European Journal of Marketing, vol. 28, no. 3, pp. 55-62.

Thompson, C., Locander, W. \& Pollio, H. 1990, 'The Lived Meaning of Free Choice: An Existential-Phenomenological Description of Everyday Consumer Experiences of Contemporary Married Women', Journal of Consumer Research, vol. 17, pp. 346-361.

Thompson, D. 1995, The Concise Oxford Dictionary, Oxford University Press, Oxford

Tian, K. \& Belk, R. 2005, 'Extended Self and Possessions in the Workplace', Journal of Consumer Research, vol. 32, pp. 297-310.

Tomlinson, H. 1989, 'After Truth: Post-modernism and the Rhetoric of Science' in Dismantling Truth: Reality in the Post-modern World, eds H. Lawson \& L. Appignanesi, Weidenfeld \& Nicolson, London.

Toulmin, S. 1977, 'From Form to Function: Philosophy and History of Science in the 1950s and Now', Daedalus, p. 156.

Tranfield, D. \& Starkey, K. 1998, 'The Nature, Social Organisation and Promotion of Management Research: Towards Policy', British Journal of Management, vol. 9, pp. 349-353.

Tsoukas, H. 2000, 'False Dilemmas in Organization Theory: Realism or Social Constructivism?', Organization, vol. 7, no. 3, pp. 531-535. 
Tsoukas, H. \& Cummings, S. 1997, 'Marginalization and Recovery: The Emergence of Aristotelian Themes in Organizations Studies', Organization Studies, vol. 18, no. 4, pp. 655-683.

Tsoukas, H. \& Hatch, M. 2001, 'Complex thinking, complex practice: The case for a narrative approach to organizational complexity', Human Relations, vol. 54, no. 8 , pp. 979-1013.

van Bruggen, G., Smidts, A. \& Wierenga, B. 1998, 'Improving decision making by means of a marketing decision support system', Management Science, vol. 44, no. 5, pp. 645-658.

Vargo, S. \& Lusch, R. 2004, 'Evolving to a New Dominant Logic for Marketing', Journal of Marketing, vol. 68, no. 1, pp1-17.

Varman, R. \& Belk, R. 2008, 'Weaving a Web: subaltern consumers, rising consumer culture, and television', Marketing Theory, vol. 8, no.3, pp. 227-252.

Vasquez, J. A. 1997, 'The Realist Paradigm and Degenerative versus Progressive Research Programs', American Political Science Review, vol. 91, no. 4, pp. 899-912.

Veloutsou, C., Saren, M. \& Tzokas, N. 2002, 'Relationship Marketing: What if?', European Journal of Marketing, vol. 36, no. 4, pp. 433-449.

Walle, A. H. 2001, 'Immanuel Kant, Marketing Theory and the Modern Temper', Management Decision, vol. 39, no. 6, pp. 426-430.

Walsh, R. 2001, 'Fabula and Fictionality in Narrative Theory', Style, vol. 35, no.4, pp. 592-606.

Waltz, K. N. 1997, 'Evaluating Theories', American Political Science Review, vol. 91, no. 4, pp. 913-917.

Warms, C. A. \& Schroeder, C. A. 1999, 'Bridging the Gulf between Science and Action: The "New Fuzzies" Neopragmatism', Advances in Nursing Science, vol. 22, no. 2, pp. 1-10.

Wartofsky, M. W. 1976, 'The Relation Between Philosophy of Science and History of Science' in Essays in Memory of Imre Lakatos, eds R. Cohen et al, Reidel Publishing, Dordrecht.

Weaver, G. R. \& Gioia, D. A. 1994, 'Paradigms Lost: Incommensurability vs Structurationist Inquiry', Organisation Studies, vol. 15, no. 4, pp. 565-590.

Weber, M. 1930, The Protestant Ethic and the Spirit of Capitalism, Allen and Unwin, London.

Webster, F. E. 1988, 'The Rediscovery of the Marketing Concept', Business Horizons, May-June, pp. 29-39.

Webster, F. E. 1992, 'The Changing Role of Marketing in the Corporation', Journal of Marketing, vol. 56, pp. 1-17.

Weick, K. 1989, 'Theory Construction as Disciplined Imagination', The Academy of Management Review, vol. 14, no. 4, pp. 516-531.

Weick, K. 1995, Sensemaking in Organizations, Sage Publications, Thousand Oaks.

Weinstein, D. \& Weinstein, M. A. 1998, 'Is Postmodern Organization Theory

Sceptical?', Journal of Management History, vol. 4, no. 4, pp. 350-362. 
Wensley, R. 1990, "'The Voice of the Consumer?": Speculations on the Limits to the Marketing Analogy', European Journal of Marketing, vol. 24, no. 7, pp. 49-60.

Wensley, R. 2002, 'A Bridge over Troubled Water?', European Journal of Marketing, vol. 36, no. 2, pp. 391-400.

White, S. K. 2000, Sustaining Affirmation: The Strengths of Weak Ontology in Political Theory, Princeton University Press, New Jersey.

Wierenga, B. \& van Bruggen, G.H. 1997, 'The Integration of Marketing ProblemSolving Modes and Marketing Management Support Systems', Journal of Marketing, vol. 61, no. 3, pp. 21-37.

Wierenga, B. 2002, 'On Academic Marketing Knowledge and Marketing Knowledge that Marketing Mangers use for Decision-making', Marketing Theory, vol. 2, no. 4, pp. 355-362.

Willmott, H. 1993, 'Paradigm Gridlock: A Reply', Organization Studies, vol. 14, no. 5, pp. 727-730.

Willmott, H. 1993a, 'Breaking the Paradigm Mentality', Organisation Studies, vol. 14, no. 5, pp. 681-719.

Willmott, H. 1999, 'On the Idolization of Markets and Denigration of Marketers: Some Critical Reflections on a Professional Paradox', in Rethinking Marketing, eds D. Brownlie et al, Sage Publications, London.

Wilson, E. \& Woodside, A. 2001, 'Executive and consumer decision processes: increasing useful sensemaking by identifying similarities and departures', The Journal of Business \& Industrial Marketing, vol. 15, no. 5, pp. 401-414.

Wittgenstein, L. 1974, Philosophical Investigations, $2^{\text {nd }}$ edition, trans G.E.M. Anscombe, Basil Blackwell, Oxford.

Woodside, A. G. 2001, 'Editorial: Sense Making in Marketing Organizations and Consumer Psychology: Theory and Practice', Psychology and Marketing, vol. 18, no. 5, pp. 415-421.

Woodside, A., Caldwell, M. \& Spurr, R. 2006, 'Advancing Ecological Systems Theory in Lifestyle, Leisure, and Travel Research', Journal of Travel Research, vol. 44 , no. 3 , p. 259

Woolgar, S. 1989, 'The Ideology of Representation and the Role of the Agent' in Dismantling Truth: Reality in the Post-modern World, eds H. Lawson \& L. Appignanesi, Weidenfeld \& Nicolson, London.

Worrall, J. 1978, 'The Ways in which the Methodology of Scientific Research Programmes Improves on Popper's Methodology' in Progress and Rationality in Science, eds R Cohen et al, Reidel Publishing, Holland.

Yankelovich, D. 1991, Coming to Public Judgment : Making Democracy Work in a Complex World, Syracuse University Press, New York.

Yearley, S. 1988, Science, Technology and Social Change, Unwin Hyman, London.

Zaltman, G., Lemasters, K. \& Heffring, M. 1982, Theory Construction in Marketing, John Wiley and Sons, New York. 
Zinkham, G. M. \& Hirschheim, R. 1992, 'Truth in Marketing Theory and Research: An Alternative Perspective', Journal of Marketing, vol. 56, pp. 80-88. 


\section{Appendices}

Appendix A: Interview Request Letter

Appendix B: Prompt Sheet

Appendix C: Airline Best Practice

Appendix D: Air New Zealand Advertising

Appendix E: Air New Zealand Kiosks 


\section{Appendix A: Interview Request Letter}

2 May 2006

\{Name\}

\{Title\}

Air New Zealand

Private Bag

Auckland

Dear XXXXX

Thank you for taking the time to participate in this study. The purpose of the interview is to gain information to be used as an exemplary case study for my $\mathrm{PhD}$ degree. The thesis is concerned with marketing as a discursive practice. In particular I am interested in your interpretation of the events leading to the introduction of the domestic Express Class service in 2002. The interview is designed to take between 60-90 minutes.

The success of this research is reliant upon your honest opinion so maintaining confidentiality is of the utmost importance. Under no circumstances will the information presented during the interview be attributed to any one individual. The organisation will be identified but your name and title will remain anonymous. Interview tapes and transcripts will be kept in a locked office, and will be destroyed at the conclusion of the research. The research findings will be published in the Victoria University library and excerpts may be included in academic publications and/or academic conferences.

Victoria University of Wellington required ethical approval prior to this research being conducted. Please note that approval from the Human Ethics Committee has been granted.

You will be given the relevant piece in the case study to read before the thesis is submitted for examination. If you for any reason would like to make contact regarding this research please contact one of the following:

David Stewart

Associate Professor Bob Gregory

Professor Stephen Cummings
044635150

David.Stewart@vuw.ac.nz

044635047 Bob.Gregory@vuw.ac.nz 044636931

Steven.Cummings@vuw.ac.nz

Yours sincerely

David Stewart

Senior Lecturer 


\section{Appendix B: Prompt Sheet}

\section{Prompt Sheet}

Interviewer:

I have written a realist account of the introduction of Express Class in the domestic market and framed it in terms of the marketing mix. The information for this case was gained from secondary sources, e.g. newspaper and magazine articles.

In contrast to this I am interested in your story your perspective on the issues surrounding the development of the strategy and the implementation of domestic Express. Casting your mind back, how did you make sense of such an important change in direction for the airline?

\section{Additional prompts:}

1. What were the main drivers for the change in the airline's marketing strategy?

2. What challenges did you face in the implementation of the strategy?

3. What factors were affecting the structure of the industry at this time?

4. What alternative models were considered when developing the new strategy?

5. How did the staff react to the new strategy?

6. How did membership of the Star Alliance impact on the development of the strategy?

7. What type of customer researcher was conducted prior and post the launch of the domestic Express class? 
8. What changes were made to the physical surroundings at the airports and aircraft to communicate the new service?

9. How did IT impact upon the architecture of Express class?

10. How was the concept of Express class service communicated to the general public?

11. What was the reaction of travel agents to the introduction of Express class? 


\section{Appendix C: Airline Best Practice}

\begin{tabular}{|c|c|c|c|c|c|}
\hline $\begin{array}{l}\text { Full-Service } \\
\text { Airlines }\end{array}$ & $\begin{array}{l}\text { Low Cost } \\
\text { Airlines }\end{array}$ & $\begin{array}{l}\text { Regional } \\
\text { Airlines }\end{array}$ & Freight Airlines & $\begin{array}{l}\text { Charter } \\
\text { Airlines }\end{array}$ & Virtual Airlines \\
\hline \multicolumn{6}{|l|}{ Description: } \\
\hline $\begin{array}{l}\text { Traditional } \\
\text { airlines that } \\
\text { offer full } \\
\text { amenities } \\
\text { (meals, } \\
\text { lounges, } \\
\text { frequent flyer } \\
\text { schemes, etc) } \\
\text { and service a } \\
\text { large network } \\
\text { of destinations }\end{array}$ & $\begin{array}{l}\text { Often offer low } \\
\text { frills and low } \\
\text { prices } \\
\text { providing value } \\
\text { for money; } \\
\text { typically } \\
\text { operate point- } \\
\text { to-point }\end{array}$ & $\begin{array}{l}\text { Usually } \\
\text { operate aircraft } \\
\text { less than } 100 \\
\text { seats; often } \\
\text { turbo-prop or } \\
\text { 'regional jet' } \\
\text { aircraft } \\
\text { servicing } \\
\text { markets within } \\
700 \text { km }\end{array}$ & $\begin{array}{l}\text { Usually only } \\
\text { carry freight } \\
\text { and not } \\
\text { passengers }\end{array}$ & $\begin{array}{l}\text { Do not operate } \\
\text { on a scheduled } \\
\text { or regular } \\
\text { basis; typically } \\
\text { offer a fixed } \\
\text { number of } \\
\text { flights on an } \\
\text { ad-hoc basis to } \\
\text { a particular } \\
\text { destination }\end{array}$ & $\begin{array}{l}\text { Typically } \\
\text { outsource } \\
\text { most functions; } \\
\text { charter aircraft } \\
\text { from other } \\
\text { operations, } \\
\text { use travel } \\
\text { agents to sell } \\
\text { seats }\end{array}$ \\
\hline \multicolumn{6}{|l|}{ Also Known as: } \\
\hline $\begin{array}{l}\text { Network } \\
\text { Carriers } \\
\text { Legacy Airlines }\end{array}$ & $\begin{array}{l}\text { Value-based } \\
\text { Carriers } \\
\text { Low Fare } \\
\text { Airlines }\end{array}$ & $\begin{array}{l}\text { Commuter } \\
\text { Airlines }\end{array}$ & $\begin{array}{l}\text { Cargo Airlines } \\
\text { Freight } \\
\text { Integrators }\end{array}$ & & \\
\hline \multicolumn{6}{|l|}{ Some Examples: } \\
\hline $\begin{array}{l}\text { Qantas } \\
\text { British Airways } \\
\text { Air France } \\
\text { American } \\
\text { Airlines }\end{array}$ & $\begin{array}{l}\text { Southwest } \\
\text { Jet Blue } \\
\text { Ryanair } \\
\text { Easyjet } \\
\text { Virgin Blue } \\
\text { Air Asia }\end{array}$ & $\begin{array}{l}\text { Tyrolean } \\
\text { Express Jet } \\
\text { Piedmont } \\
\text { Airlines } \\
\text { Regional } \\
\text { Express }\end{array}$ & $\begin{array}{l}\text { Fed Ex } \\
\text { Atlas Air Cargo } \\
\text { UPS } \\
\text { Evergreen } \\
\text { International }\end{array}$ & $\begin{array}{l}\text { National Jet } \\
\text { Systems } \\
\text { Air Tours } \\
\text { World Airways }\end{array}$ & Jet America \\
\hline
\end{tabular}

Source: IAS Aviation Consulting 
Appendix D: Air New Zealand Advertising

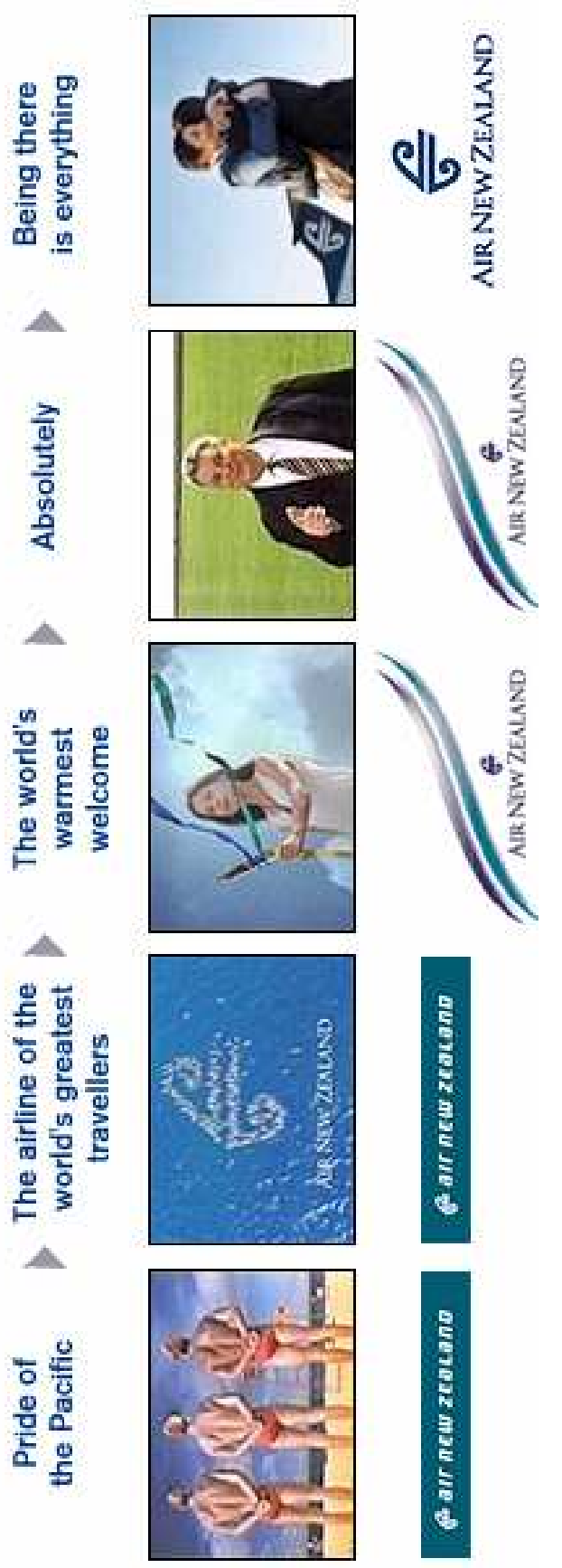


Appendix E: Air New Zealand Kiosks

Air New Zealand ExpressCheck Uptake
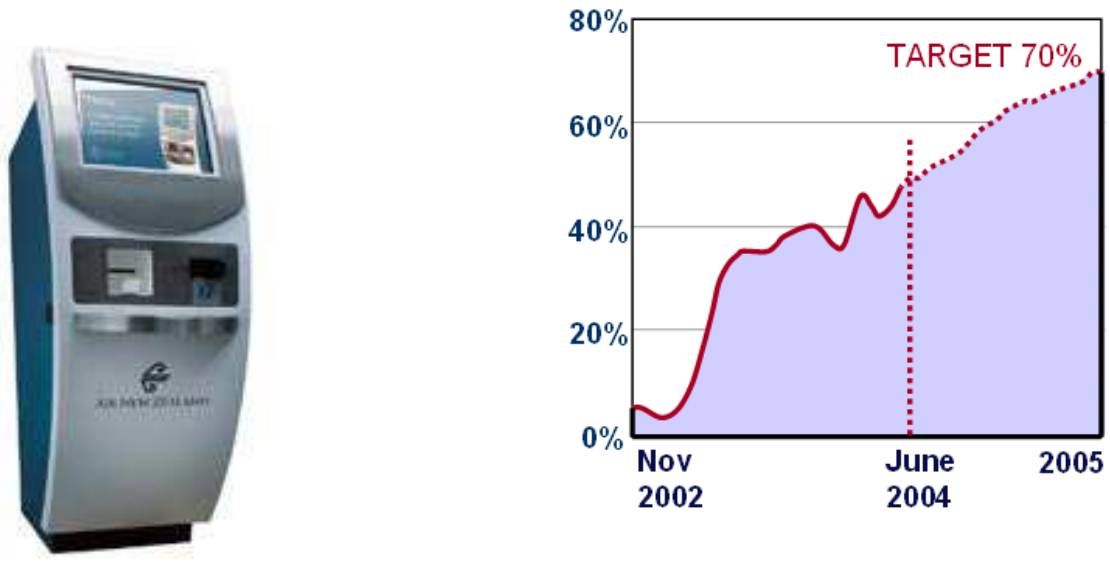

Page 365 